\title{
A Multi-Agent Design for Smart Distribution Automation System with Distributed Energy Resources and Microgrids
}

Sridhar Chouhan

Follow this and additional works at: https://researchrepository.wvu.edu/etd

\section{Recommended Citation}

Chouhan, Sridhar, "A Multi-Agent Design for Smart Distribution Automation System with Distributed Energy Resources and Microgrids" (2017). Graduate Theses, Dissertations, and Problem Reports. 5363. https://researchrepository.wvu.edu/etd/5363

This Dissertation is protected by copyright and/or related rights. It has been brought to you by the The Research Repository @ WVU with permission from the rights-holder(s). You are free to use this Dissertation in any way that is permitted by the copyright and related rights legislation that applies to your use. For other uses you must obtain permission from the rights-holder(s) directly, unless additional rights are indicated by a Creative Commons license in the record and/ or on the work itself. This Dissertation has been accepted for inclusion in WVU Graduate Theses, Dissertations, and Problem Reports collection by an authorized administrator of The Research Repository @ WVU.

For more information, please contact researchrepository@mail.wvu.edu. 


\title{
A Multi-Agent Design for Smart Distribution Automation System with Distributed Energy Resources and Microgrids
}

\section{Sridhar Chouhan}

Dissertation submitted to the

Benjamin M. Statler College of Engineering and Mineral Resources at West Virginia University

in partial fulfillment of the requirements for the degree of

Doctor of Philosophy in Electrical Engineering

\author{
Ali Feliachi, Ph.D., Chair \\ M. A. Choudhry, Ph.D. \\ Sarika Khushalani Solanki, Ph.D. \\ Powsiri Klinkhachorn, Ph.D. \\ Daryl S Reynolds, Ph.D. \\ Hong-Jian Lai, Ph.D.
}

Lane Department of Computer Science and Electrical Engineering

Morgantown, West Virginia

2017

Keywords: Multi-Agent System, Distribution Automation, Fault Location and Isolation, Service Restoration, Distributed Energy Resources, Microgrids, Reinforcement Learning, Switch Optimization 


\title{
ABSTRACT
}

\section{A Multi-Agent Design for Smart Distribution Automation System with Distributed Energy Resources and Microgrids}

\author{
Sridhar Chouhan
}

The proliferation of Smart Grid technologies such as distributed energy resources (DER) and microgrids has resulted in new opportunities and challenges to existing Distribution Automation (DA) solutions. A novel hybrid Multi-Agent System (MAS) Fault Location, Isolation, and Restoration (FLIR) solution is proposed for electric distribution systems with different types of distributed generation (DG) resources and microgrids. The main goal of the MAS is to locate and isolate the fault and then to automatically reenergize fault-free portions of the network to restore power to as many customers as possible while maintaining system constraints like voltage and thermal capacity limits.

Determination of the optimum number and placement of automated switches is an important and a daunting task in the economic feasibility process of any DA project. An innovative algorithm based on relative reduction in the normalized customer interruption costs is presented for the switch optimization problem. The proposed approach isolates the impacts of varying switch investment and customer interruption costs that are usually based on outdated surveys.

A distributed Fault Detection algorithm is presented to support sectionalizing switch agents (SSA) to autonomously detect the fault condition and type of the fault by using the local data such as voltage and current phasor measurements. Fault characteristics of various DG systems including inverter based DG, synchronous generator, and induction generator are considered in the proposed algorithm. Fault isolation and service restoration functionalities are achieved through coordinated communications among the agents using both centralized and decentralized control strategies. Feeder Agent (FA) uses the proposed "TieSwitch Ranking Algorithm" and "Zone Priority Algorithm" to solve the service restoration problem. A new reinforcement agent learning mechanism based on $Q$-learning is introduced to support FAs with service restoration task.

The proposed MAS is designed to be demonstrated on Mon Power, a FirstEnergy company, distribution system as part of the DOE project, West Virginia Super Circuit (WVSC). The actual distribution network is simulated using $\mathrm{CYME}^{\circledR}$, Matlab ${ }^{\circledR} / \mathrm{Simpower}^{\circledR}$, and $\mathrm{PSCAD}^{\circledR}$ software, whereas the MAS and its communications are simulated using Matlab ${ }^{\circledR}$ S-functions. The switch optimization results show that the proposed iterative algorithm can drastically reduce the search space, and can find optimal number and placement of the switches with minimum computational effort. The FLIR simulation results show promising advantages of using the proposed MAS solution with the agent learning capabilities for fault location and service restoration tasks. 
The only living gods on earth are,

'Parents' 


\section{Acknowledgements}

After several years of my PhD program, I have learned one thing - I could never have completed my dissertation, without the support and encouragement of many people around me. First of all, I would like to thank my esteemed mentor and advisor, Dr. Ali Feliachi. He has given me a chance to continue my work in the field of power systems to pursue my passion. It was a blessing to be one of his students and be able to conduct research with him. I would also like to thank rest of my committee, Dr. Muhammad A. Choudhry, Dr. Sarika Khushalani Solanki, Dr. Powsiri Klinkhachorn, Dr. Daryl S Reynolds, and Dr. Hong-Jian Lai for their constructive comments. My sincere thanks go to Dr. Kevin Milans for his feedback. Their guidance helped me immensely to shape and improve my research as well as my dissertation document.

I would like to extend my thanks to Mon Power, a FirstEnergy Company, especially to Mr. Harley Mayfield, for providing the distribution system data to help with my research work.

I would like to thank my family and in-laws for their unending support and love. Without their support, I couldn't have made it through this process. My sincere gratitude goes to my cousin for helping and motivating me. I can never thank enough to my loving wife for all her support and understanding, she sacrificed countless weekends to help me get this degree. My humble pranam goes to the almighty god for being always with me as a guiding light.

Lastly, Grand Pa, I miss you so much!!! 


\section{Contents}

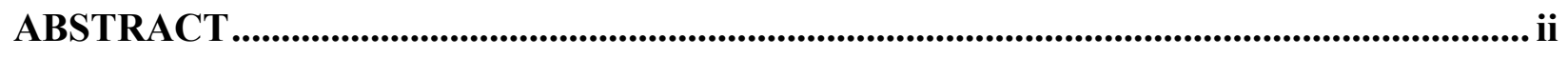

Acknowledgements ............................................................................................................................. iv

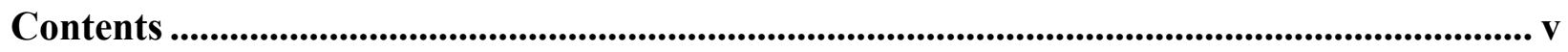

List of Figures............................................................................................................................... ix

List of Tables .................................................................................................................................................... xii

Nomenclature ........................................................................................................................... xiii

Symbols ...................................................................................................................................... xiv

Chapter 1 Introduction ............................................................................................................... 1

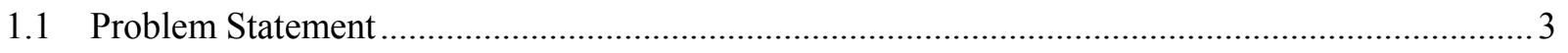

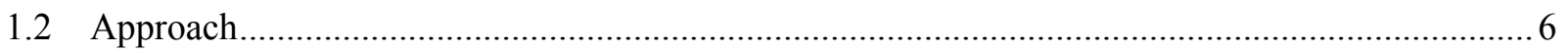

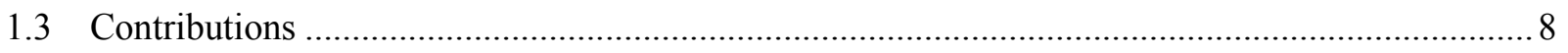

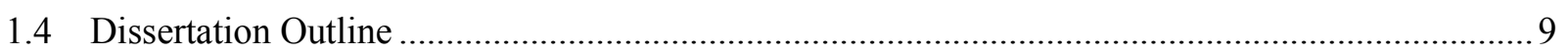

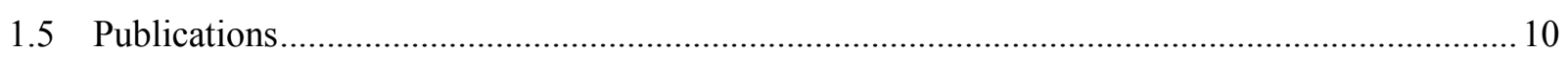

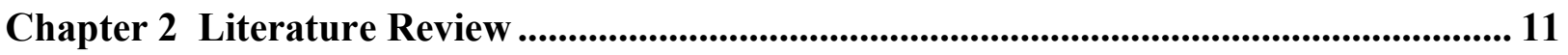

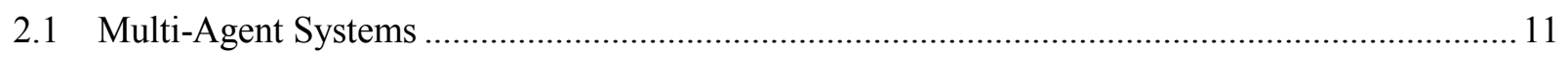

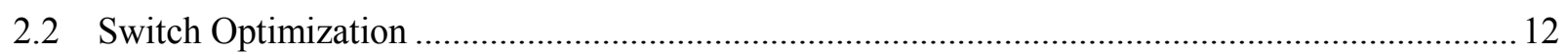

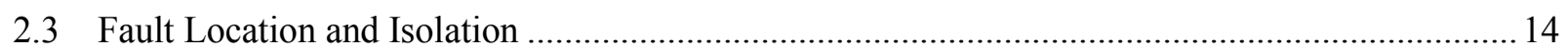

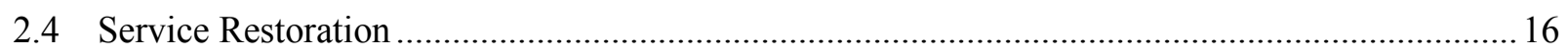

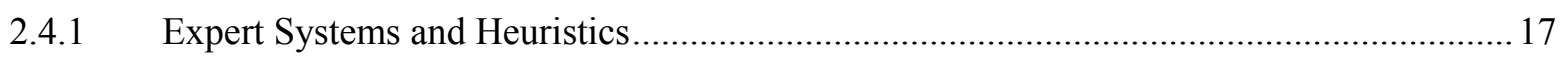

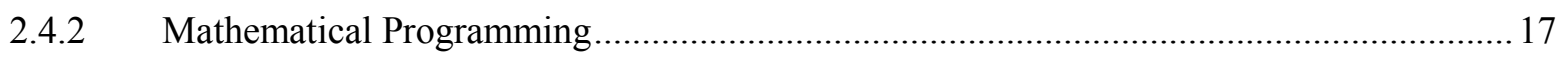

2.4.3 Multi-Agent System technology …………………................................................... 18

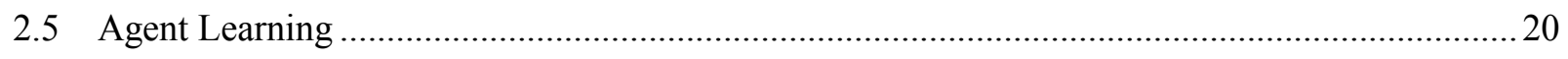

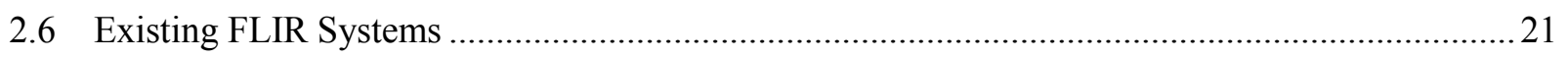

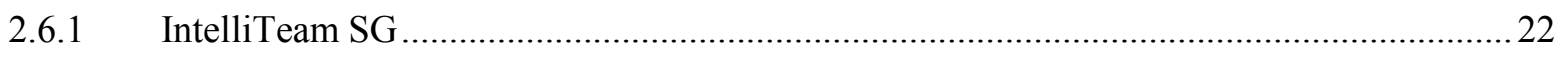

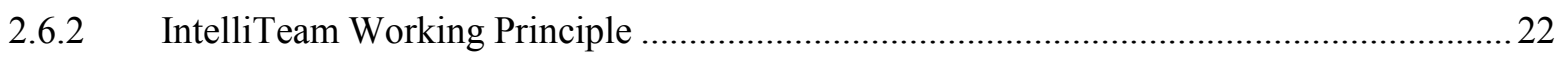

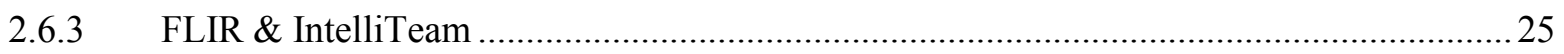


Chapter 3 Multi-Agent Systems ........................................................................................... 26

3.1 Agent Communication ............................................................................................. 30

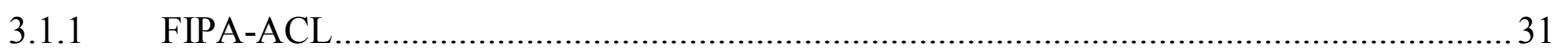

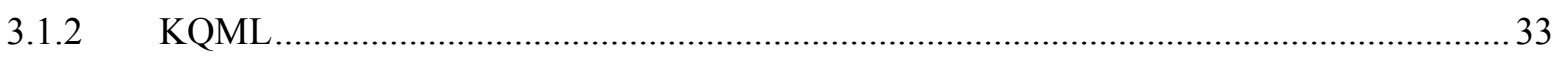

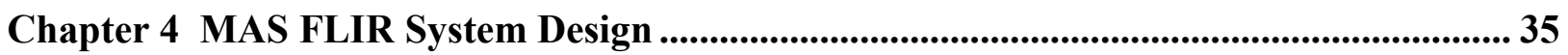

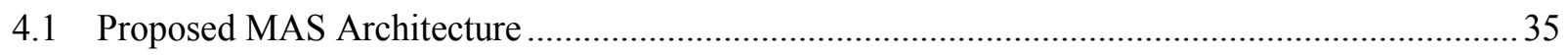

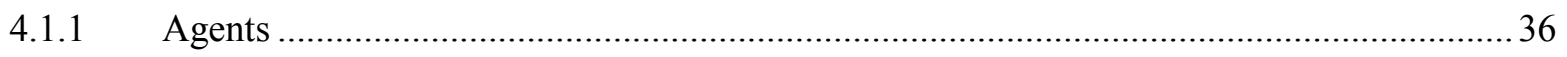

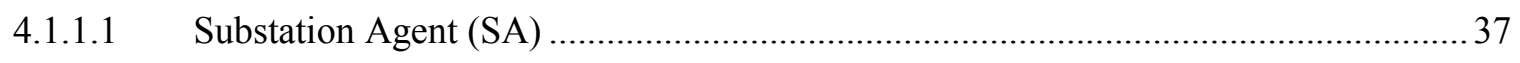

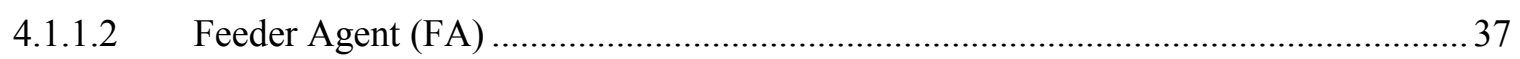

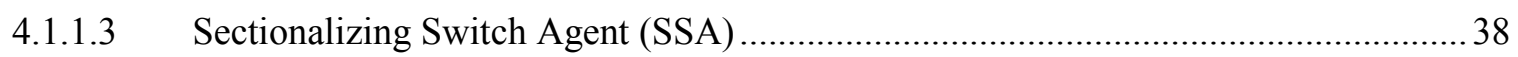

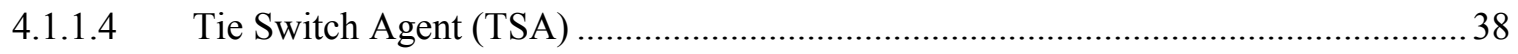

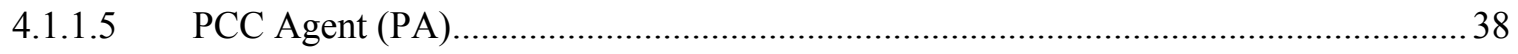

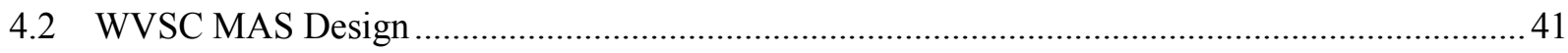

4.3 Agent State Transition .......................................................................................... 42

4.4 Proposed FLIR Process Flow................................................................................... 43

Chapter 5 Switch Optimization ................................................................................................. 45

5.1 Mathematical Problem Formulation ………………………………………………………...... 46

5.2 Iterative Algorithm for Optimal Switch Number and Placement .................................................. 47

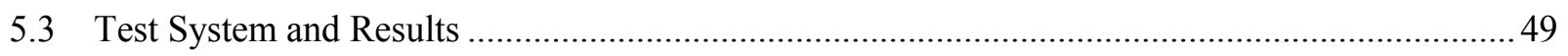

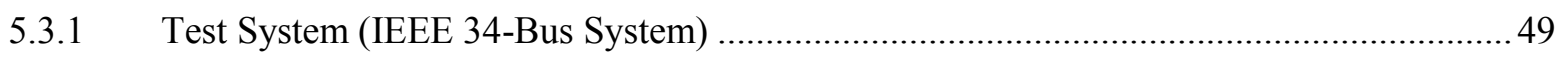

5.3.2 Test System (IEEE 123-Bus System) ………………………………………..... 51

5.3.3 Test System (WVSC Distribution System) …………............................................. 51

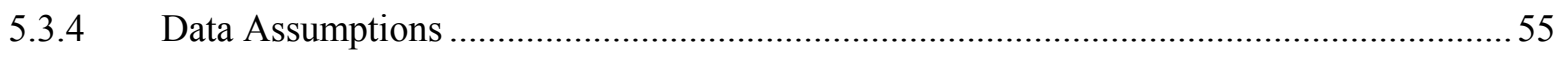

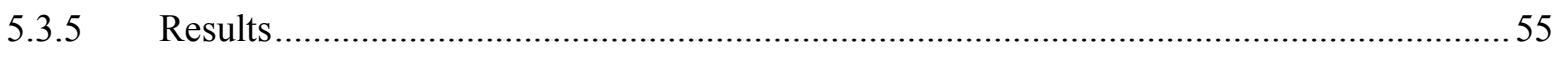

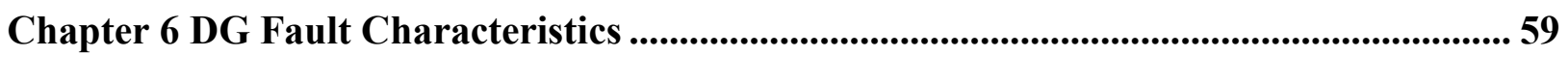

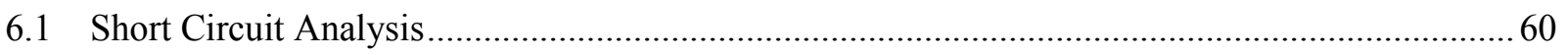

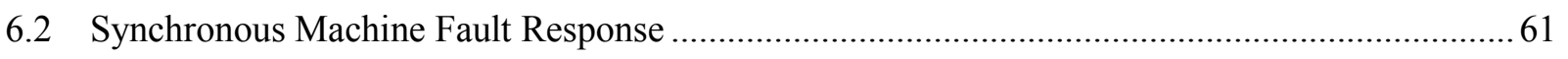

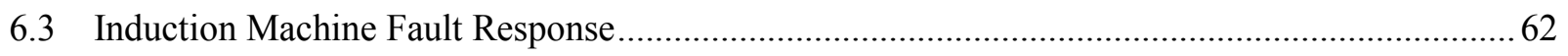

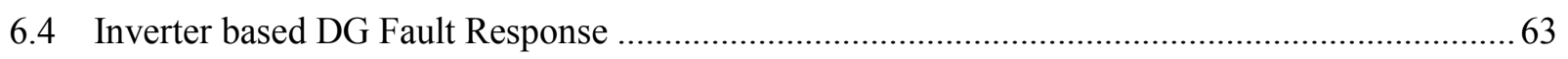

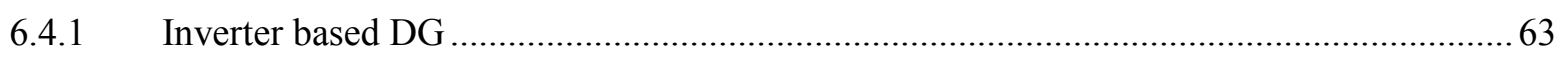

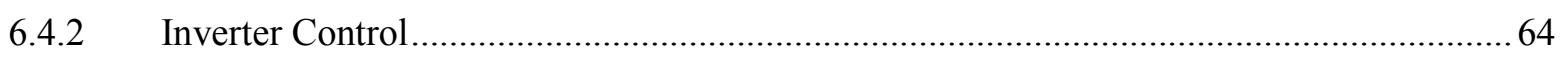

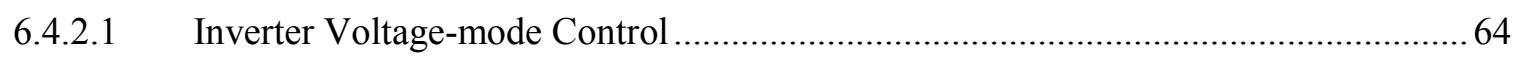

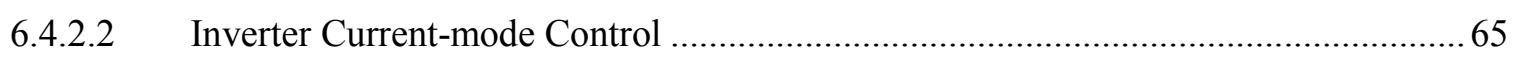

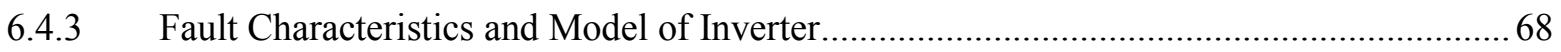

Chapter 7 MAS Fault Location \& Isolation .................................................................................... 71 


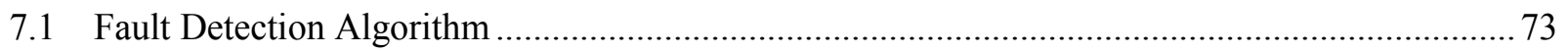

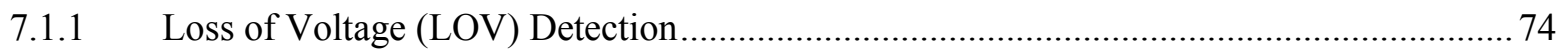

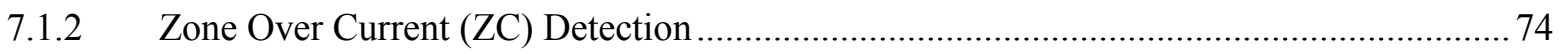

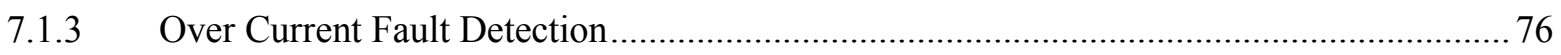

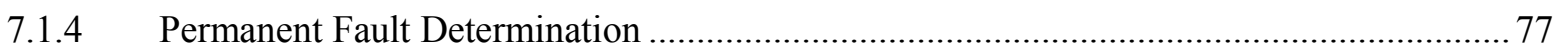

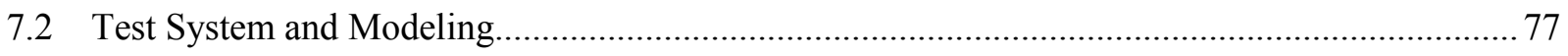

7.2.1 West Virginia Super Circuit (Base Case)..................................................................... 78

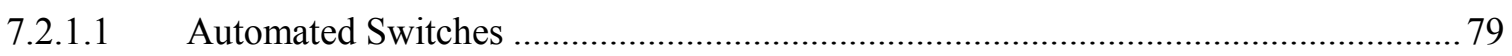

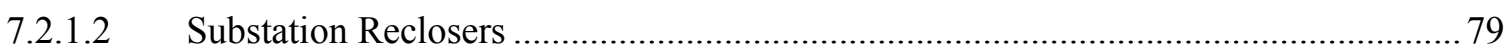

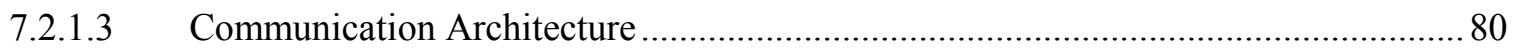

7.2.2 West Virginia Super Circuit (FLI Test Case) ................................................................. 81

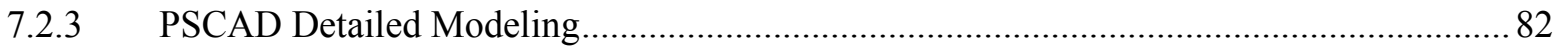

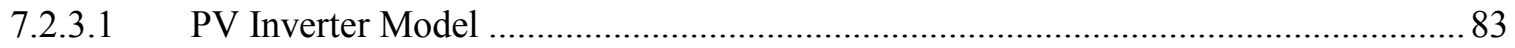

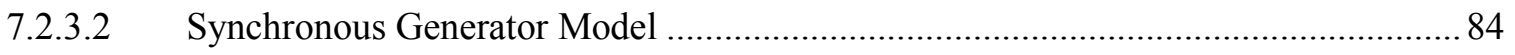

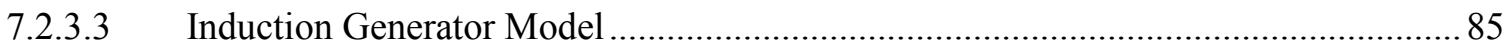

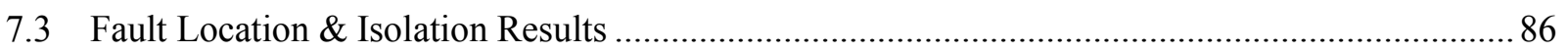

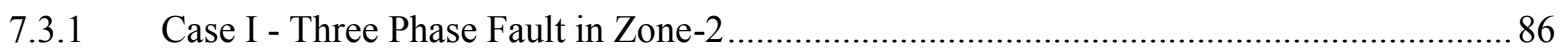

7.3.2 Case II - Line to Ground (LG-A) Fault in Zone-1 ....................................................... 93

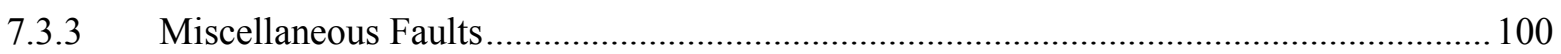

Chapter 8 MAS Service Restoration ............................................................................. 102

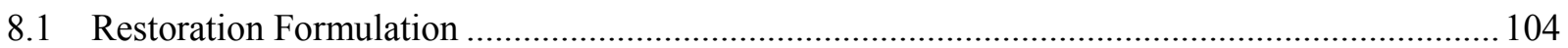

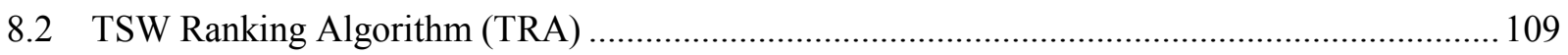

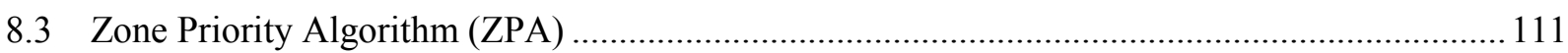

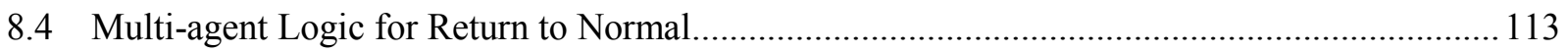

Chapter 9 Reinforcement Agent Learning .......................................................... 115

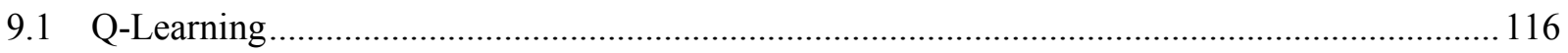

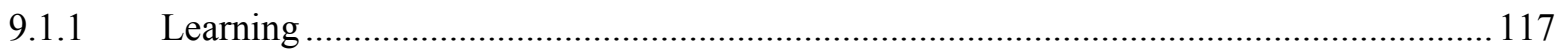

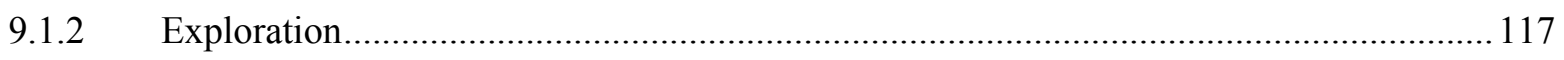

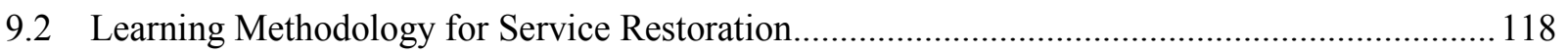

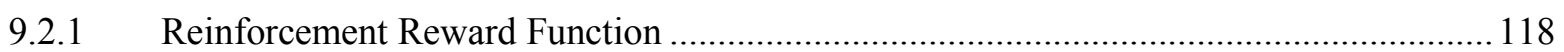

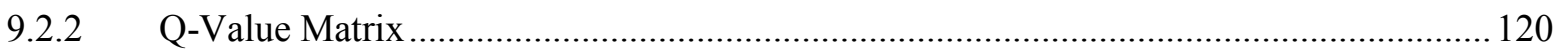

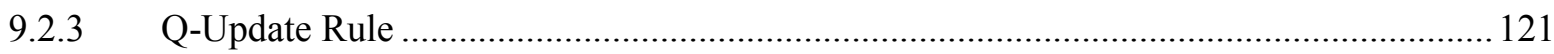

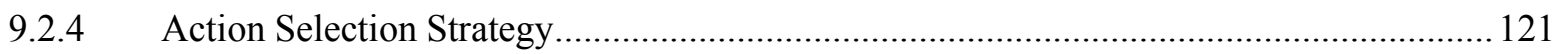

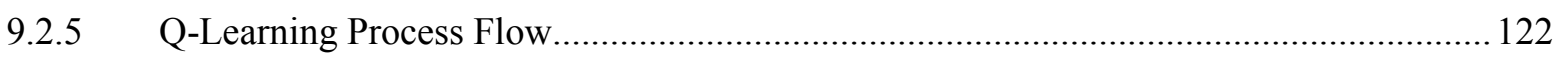

Chapter 10 Service Restoration Results .................................................................................... 124 


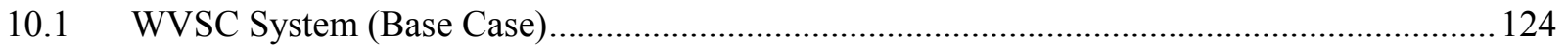

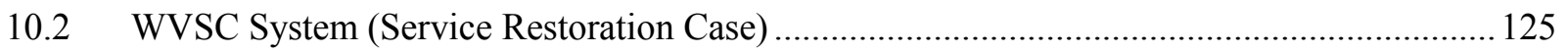

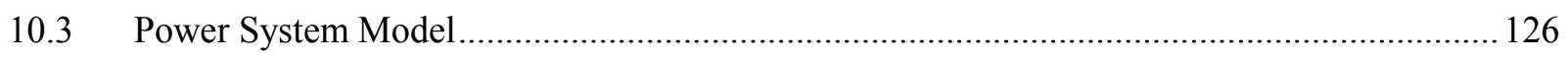

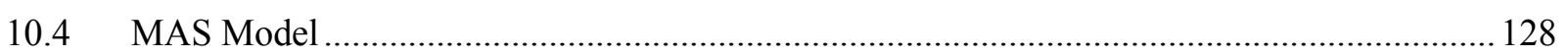

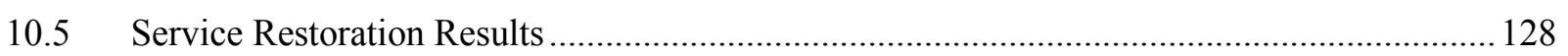

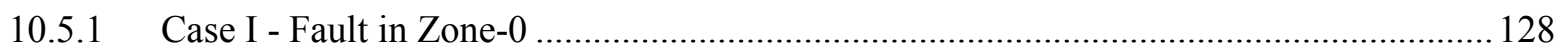

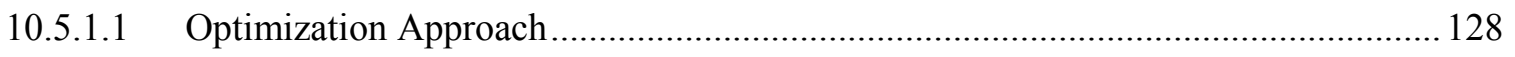

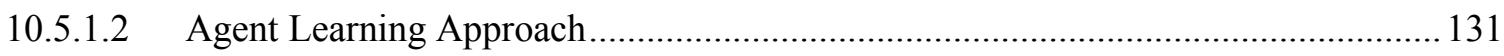

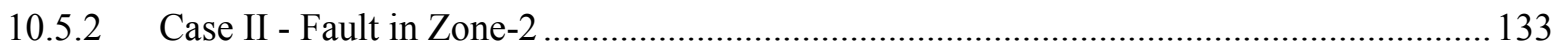

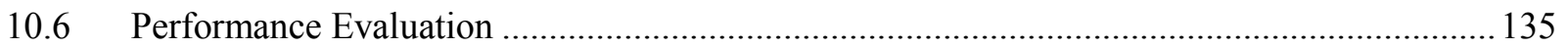

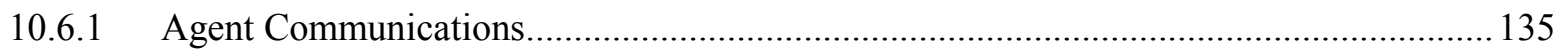

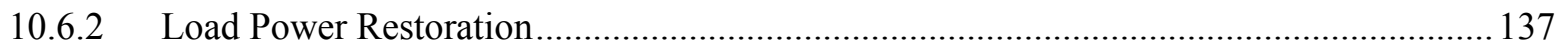

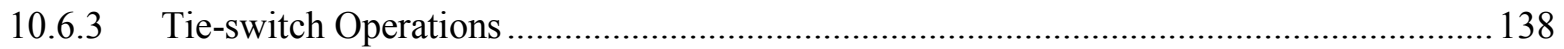

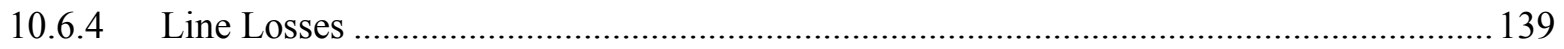

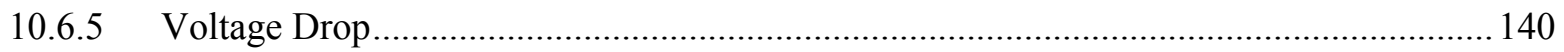

Chapter 11 Conclusions and Future Work ................................................................ 142

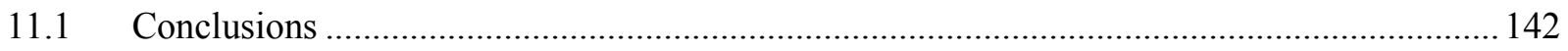

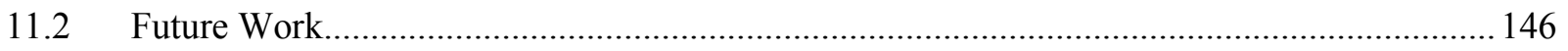

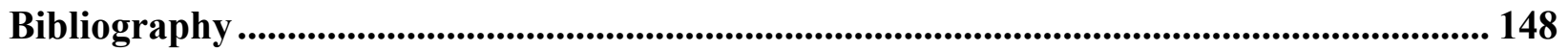




\section{List of Figures}

Figure 1-1: Outage Restoration without and with Distribution Automation ............................................ 3

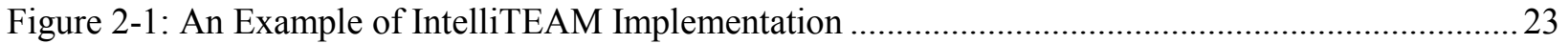

Figure 3-1: Multi-Agent System for Electric Distribution System .......................................................27

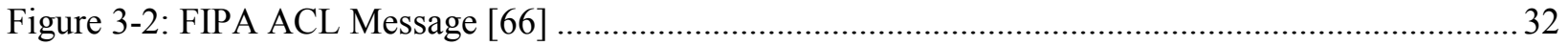

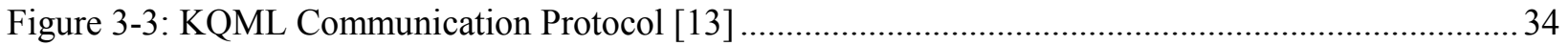

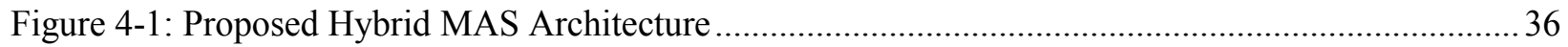

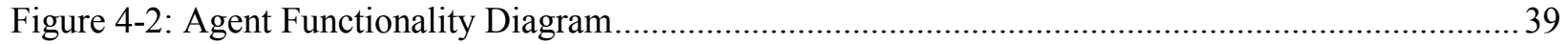

Figure 4-3: Agent Placement for WVSC Distribution System ........................................................... 41

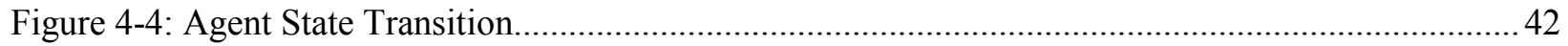

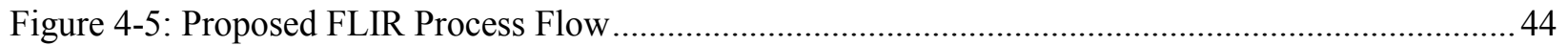

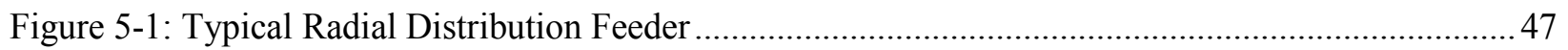

Figure 5-2: Iterative Algorithm for Optimal Switch Number and Placement........................................... 48

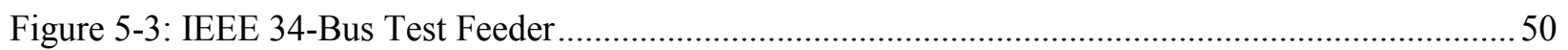

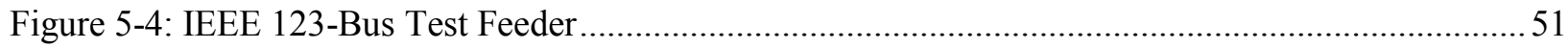

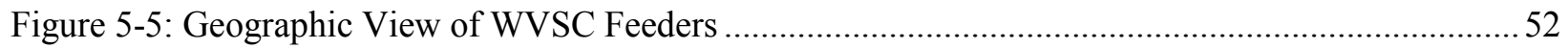

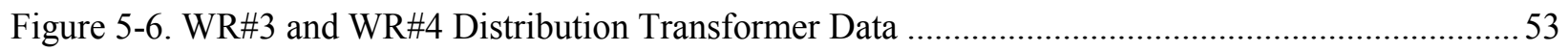

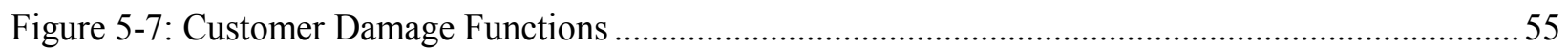

Figure 5-8: Calculated Indices for IEEE 34-Bus, IEEE-123 Bus, WR\#3, and WR\#4 Feeders ..................56

Figure 5-9: Comparison of CIC Calculations between Traditional [67] and Proposed Approaches .......... 58

Figure 6-1: Circuit Model for Asymmetrical Fault Current..................................................................... 60

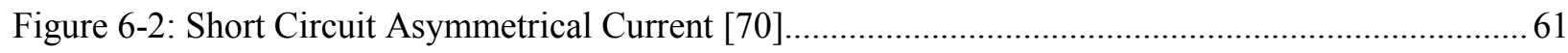

Figure 6-3: Synchronous Machine Response to Three Phase Fault .......................................................62

Figure 6-4: Induction Machine Response to Three Phase Fault ..........................................................6 63

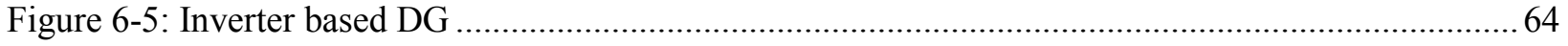

Figure 6-6: Grid-connected Inverter Voltage-mode Control .................................................................65

Figure 6-7: Grid-connected Inverter Current-mode Control.................................................................... 66 
Figure 6-8: Power Controller Implementation.

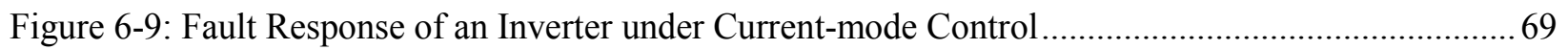

Figure 6-10: Equivalent Circuit of Grid-connected Inverter with Current Limiting ................................ 70

Figure 6-11: Equivalent Sequence Network of Grid-connected Inverter ............................................... 70

Figure 7-1: Agent Communication for Fault Location and Isolation...................................................... 72

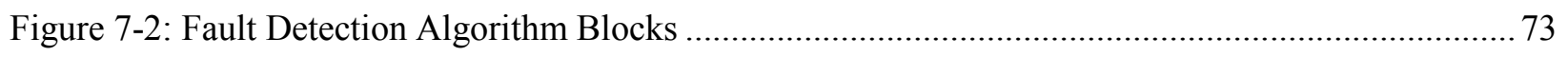

Figure 7-3: Fault Detection Algorithm - Voltage and Zone Current Change Waveforms .........................76

Figure 7-4: AND Gate Implementation of Over Current Fault Detection ............................................... 77

Figure 7-5: Single Line Diagram of WVSC Project $12.5 \mathrm{kV}$ Circuits..................................................... 78

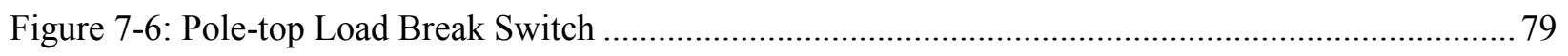

Figure 7-7: Recloser with Cooper Form-6 Controller at WestRun Substation ........................................ 80

Figure 7-8: Simplified One Line Diagram of WVSC Feeders for MAS FLI ........................................... 82

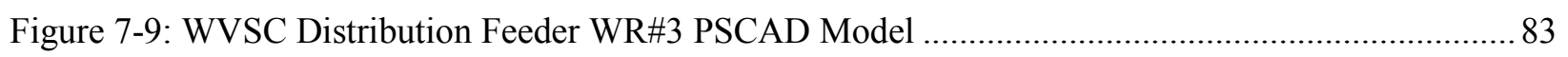

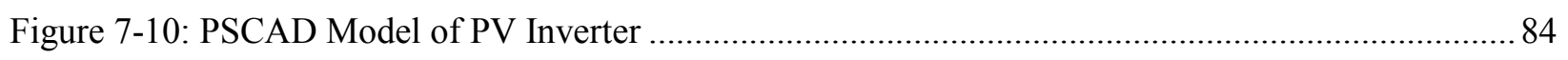

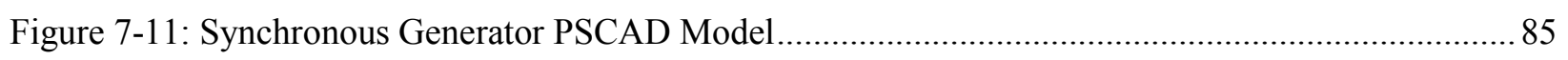

Figure 7-12: Induction Generator PSCAD Model ............................................................................ 86

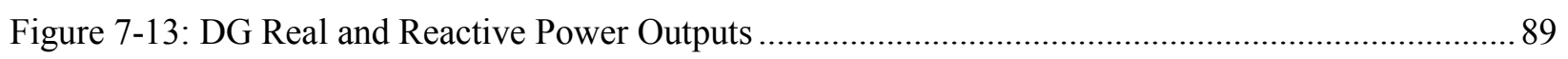

Figure 7-14: DG Instantaneous Fault Contributions ........................................................................... 90

Figure 7-15: SSW3 Measurements (Voltages, Zone Current Change, Fault Flag, and Permanent Fault

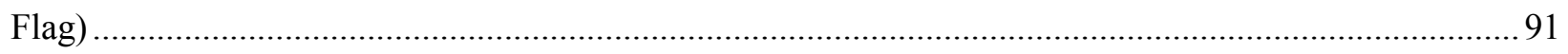

Figure 7-16: Instantaneous Voltages at the Substation and SSW locations............................................92

Figure 7-17: Agent Communications for Fault Location and Isolation for LLL Fault in Zone-2 .............93

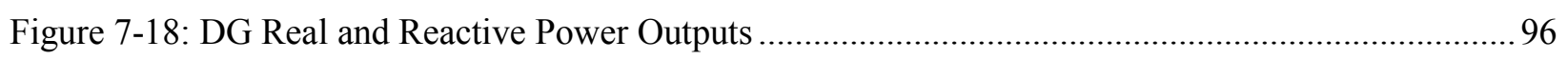

Figure 7-19: DG Instantaneous Fault Contributions ........................................................................... 97

Figure 7-20: SSW1 Measurements (Voltages, Zone Current Change, Fault Flag, and Permanent Fault

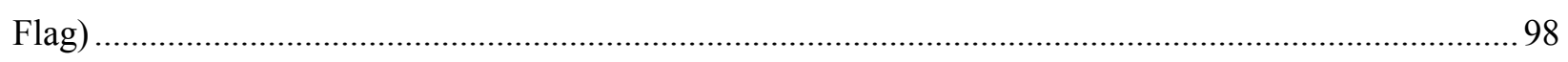

Figure 7-21: Instantaneous Voltages at the Substation and SSW locations...........................................99

Figure 7-22: Agent Communications for Fault Location and Isolation for LG-A Fault in Zone-1 .......... 100

Figure 8-1: Typical Radial Distribution Feeder with Super Zones ....................................................... 103

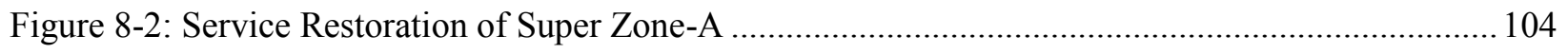

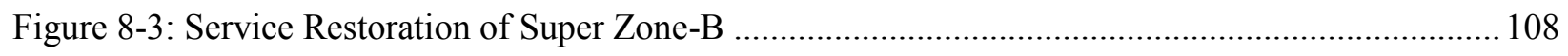

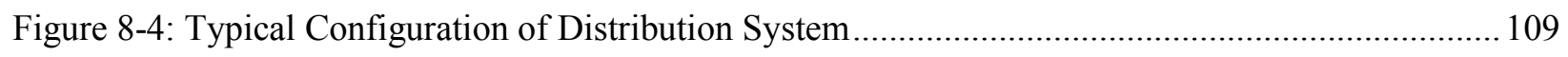

Figure 8-5: Zone Energizing Sequence using Zone Priority Algorithm. ............................................... 112

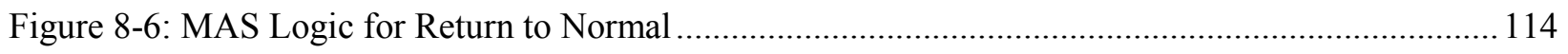


Figure 9-1: $Q$-Learning Process Flow for Service Restoration......................................................... 123

Figure 10-1: Simplified One Line Diagram of WVSC Feeders for MAS Service Restoration ................. 126

Figure 10-2: CYME $^{\circledR}$ and Matlab ${ }^{\circledR}$ Model for WVSC Project Feeders .................................................. 127

Figure 10-3: Agent Communications for Service Restoration for Zone-0 Fault ..................................... 131

Figure 10-4: Service Restorations for Case I and Case II ..................................................................... 134 


\section{List of Tables}

Table 2-1: SWOT Matrix for Current Agent Applications to Power Systems [14]................................ 12

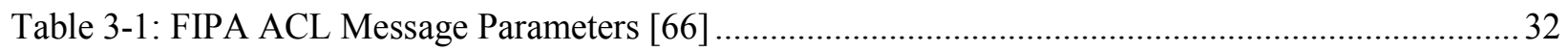

Table 4-1: Agent Knowledge/Capabilities and Behaviors....................................................................... 39

Table 5-1: IEEE 34-Bus and 123-Bus Test Feeder SS Data ................................................................50

Table 5-2. Reliability Indices of WVSC Circuits (Major Events Included) ...........................................53

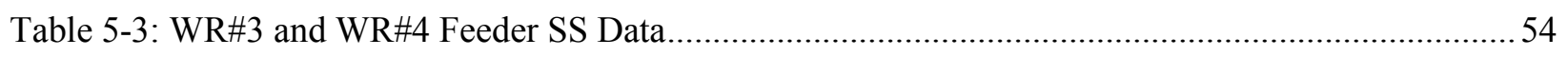

Table 5-4: Calculated Indices for IEEE 34-Bus, IEEE-123 Bus, WR\#3, and WR\#4 Feeders....................57

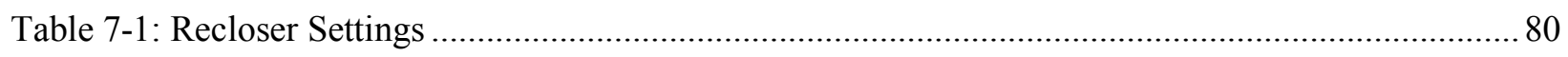

Table 7-2: Interconnection System Response to Abnormal Voltages (rms Voltage) ............................... 84

Table 7-3: Interconnection System Response to Abnormal Frequencies ............................................... 84

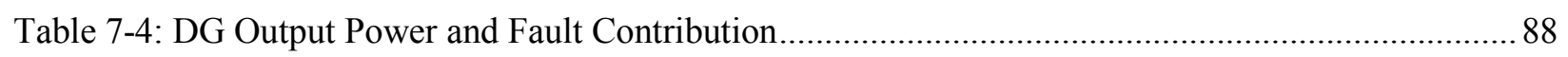

Table 7-5: Fault Counter Value and Permanent Fault Flag Status for LLL Fault in Zone-2 ..................... 88

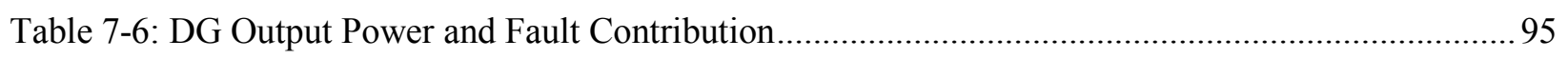

Table 7-7: Fault Counter Value and Permanent Fault Flag Status for LG-A fault in Zone-1 ....................95

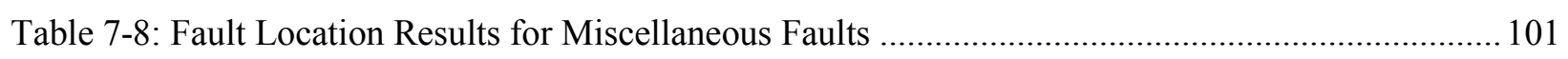

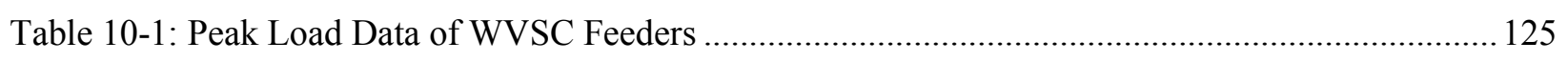

Table 10-2: Zone Pre-fault Loadings and Generation Values in $\mathrm{CYME}^{\circledR}$........................................... 129

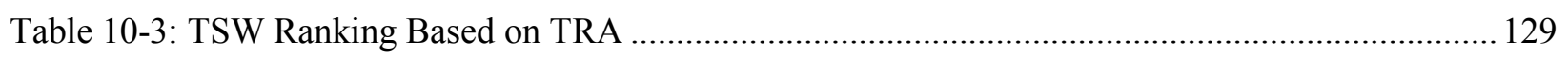

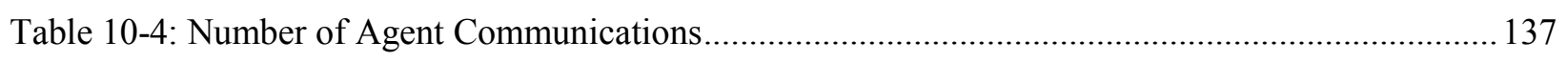

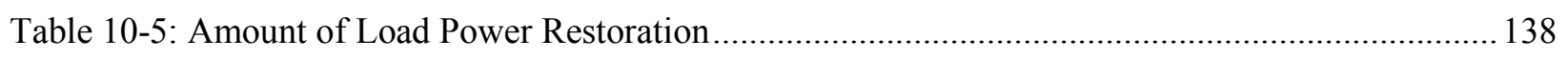

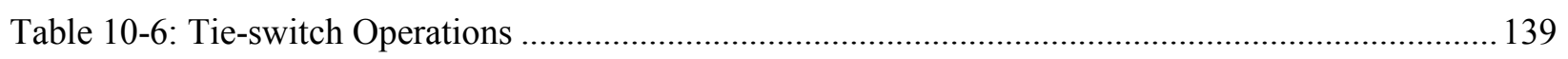

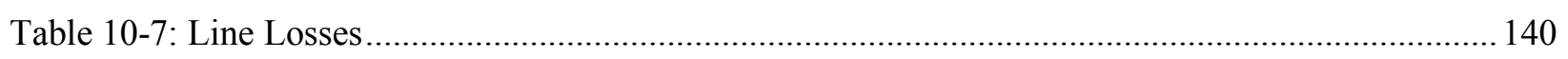

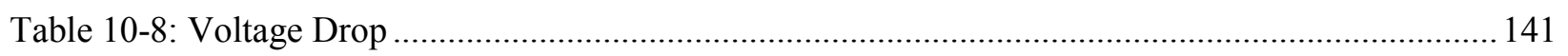




\section{Nomenclature}

$\begin{array}{ll}\text { ACL } & \text { Agent Communication Language } \\ \text { CAIDI } & \text { Customer Average Interruption Duration Index } \\ \text { CB } & \text { Circuit Breaker } \\ \text { CC } & \text { Current Change } \\ \text { CIC } & \text { Customer Interruption Cost } \\ \text { CMI } & \text { Customer Minute Interruptions } \\ \text { DA } & \text { Distribution Automation } \\ \text { DOE } & \text { Department of Energy } \\ \text { DG } & \text { Distributed Generation } \\ \text { EDS } & \text { Electric Distribution System } \\ \text { FA } & \text { Feeder Agent } \\ \text { FLI } & \text { Fault Location, Isolation } \\ \text { FLIR } & \text { Fault Location, Isolation \& Restoration } \\ \text { KQML } & \text { Knowledge Query and Manipulation Language } \\ \text { LOV } & \text { Loss of Voltage } \\ \text { MAS } & \text { Multi Agent System } \\ \text { NETL } & \text { National Energy Technology Laboratory } \\ \text { NEZ } & \text { Non-Energized Zone } \\ \text { OC } & \text { Over-Current } \\ \text { OLTC } & \text { On-Load Tap Changers } \\ \text { PA } & \text { PCC Agent } \\ \text { PCC } & \text { Point of Common Coupling } \\ \text { SA } & \text { Substation Agent } \\ \text { SAIDI } & \text { System Average Interruption Duration Index } \\ \text { SAIFI } & \text { System Average Interruption Frequency Index } \\ \text { SCADA } & \text { Supervisory Control and Data Acquisition } \\ \text { SSA } & \text { Sectionalizing Switch Agent } \\ \text { SSW } & \text { Sectionalizing Switch } \\ \text { TCC } & \text { Time Current Characteristic } \\ \text { TSA } & \text { Tie Switch Agent } \\ \text { TSW } & \text { Tie Switch } \\ \text { TRA } & \text { Tie-Switch Ranking Algorithm } \\ \text { WVSC } & \text { West Virginia Super Circuit } \\ \text { ZPA } & \end{array}$




\section{Symbols}

$\begin{array}{lll}V & \text { voltage } & \mathrm{V}(\text { Volt }) \\ I & \text { current } & \mathrm{A}(\text { Ampere }) \\ P & \text { active power } & \mathrm{W}(\mathrm{J} / \mathrm{s}) \\ Q & \text { reactive power } & \mathrm{var} \\ t & \text { time } & \mathrm{s}(\text { second }) \\ \xi & \text { failure rate } & \text { failures/mile-year } \\ f & \text { frequency } & \mathrm{Hz} \\ \beta & \text { load priority } & \\ X^{\prime} & \text { transient reactance } & \Omega(\mathrm{Ohms}) \\ X^{\prime \prime} & \text { sub-transient reactance } & \Omega(\mathrm{Ohms}) \\ a & \text { action } & \\ S & \text { state } & \\ \alpha & \text { learning rate } & \\ R & \text { reward function } & \end{array}$




\section{Chapter 1}

\section{Introduction}

According to the U.S. Department of Energy (DOE), the "Smart grid" generally refers to a class of technology that brings utility electricity delivery systems into the $21^{\text {st }}$ century, using computerbased remote control and automation [1]. The National Energy Technology Laboratory (NETL) in the United States has presented seven principle characteristics of a smart grid [2]. The smart grid should be able to heal itself after a power system event; it should enable active customer participation, resist attacks, provide power quality for the $21^{\text {st }}$ century needs, accommodate all generation and storage options, enable markets, and optimize asset utilization and operate efficiently. Among them "Self-healing" is the key characteristic. "Self-healing" in the literature was also interpreted as a technology that enables the faulty part of a system to be isolated and, ideally, restored to normal operation with little or no human intervention.

Supplying reliable and high quality electric power to customers is the highest priority of electric utilities. Electric utilities employ smart technologies and advanced communications to monitor and control the transmission and generation facilities, whereas the distribution systems are still catching up with that level of sophistication. This was mainly due to the un-justified economics of deploying automation technologies on the Electric Distribution System (EDS).

According to the DOE, more than $80 \%$ of outages are directly related to distribution systems which cost the nation more than 80 billion dollars annually [3]. Utility companies are concerned about distribution outages and its impact on their customers. Distribution system automation technologies, especially the automated Fault Location, Isolation, and Restoration (FLIR) systems 
can help to improve the overall system reliability and operational efficiency by minimizing the duration and frequency of outages.

Although manual methods of fault restoration have been used for years to provide certain levels of reliability in the EDS, automated FLIR systems can provide increased levels of reliability. Even in today's advanced world, most of the electric utilities still depend on customer calls to find out outages in their systems. The field crews are dispatched to patrol the faulted lines to find out the exact fault location and fix the fault. This is a manual intensive process and takes hours together for fault location and isolation activities as crews sometimes have to travel tens of miles and that to in inclement weather conditions most of the times. This is a poor way of handling fault situations and field crews. These inefficient manual operations can cause increased outage durations and number of outages, which can significantly hinder the system reliability.

The automated FLIR systems that exist today can locate and isolate the fault, and reconfigure the un-faulty portion of the circuits within a couple of minutes. These FLIR systems can totally be automated and do not require any human intervention to run. This will significantly improve the system reliability and the relevant reliability indices (SAIFI, SAIDI, and CAIDI). Figure 1-1 illustrates how a typical outage restoration scenario might progress both with and without advanced distribution automation [4]. The times shown will be extended even further during storm conditions when dispatchers are juggling multiple outage investigations. Feeder automation is the essential requirement of automated FLIR systems. The major components of feeder automation are Supervisory Control and Data Acquisition (SCADA), automated Sectionalizing Switches (SSW) and Tie Switches (TSW), reclosers, and advanced communications. 


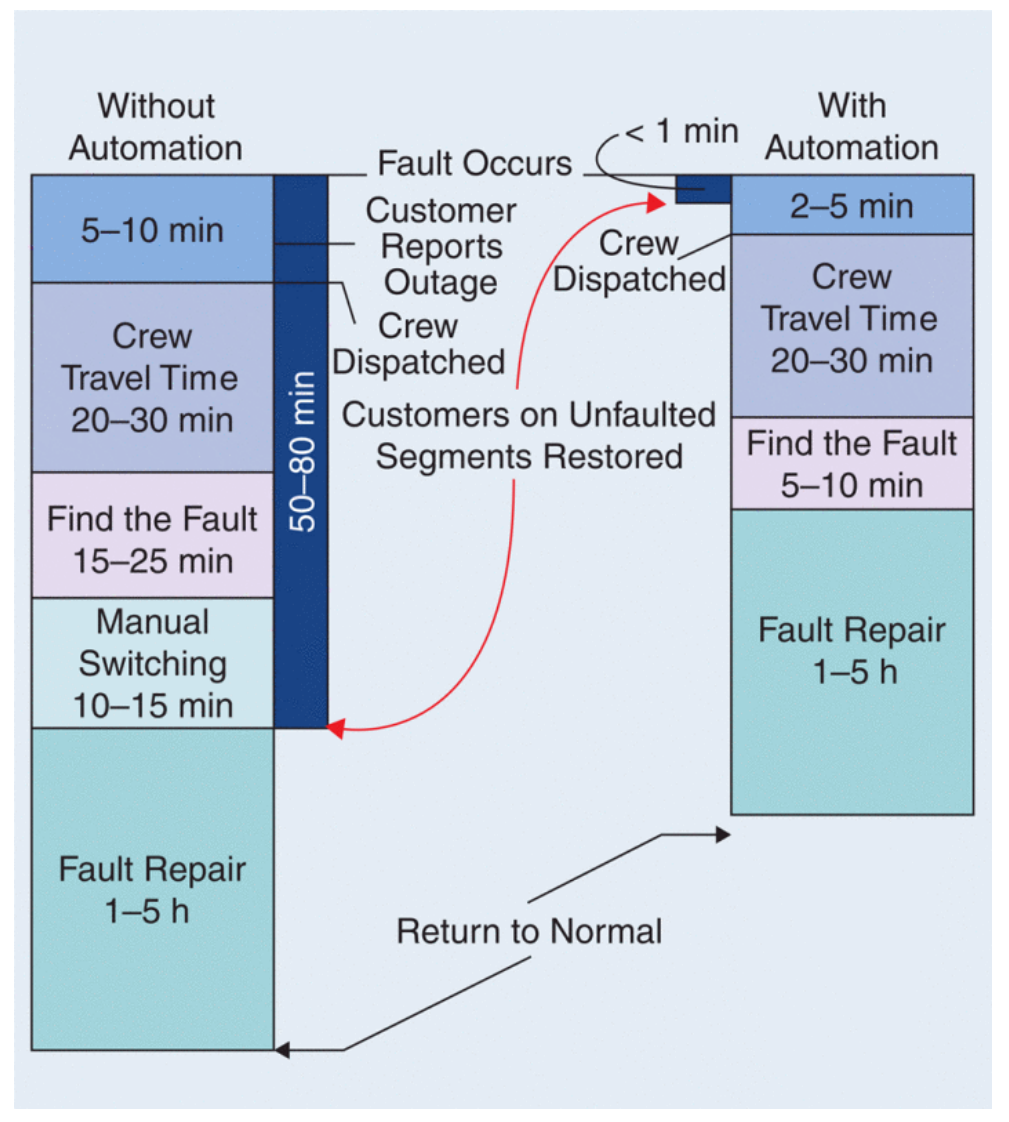

Figure 1-1: Outage Restoration without and with Distribution Automation

\subsection{Problem Statement}

Distribution self-healing through FLIR systems is not a new concept, but previous system designs must be re-evaluated to ensure proper operation with the new technologies on the distribution systems such as distributed energy resources including microgrid systems, intelligent demand control, and Volt/VAr control systems. These new technologies pose new challenges and offer new opportunities for improved performance of FLIR systems during emergencies.

Electric distribution facilities are routinely loaded to more than 50 percent of rated capacity, and sufficient capacity may not be available through adjacent feeders when needed. Hence, the recent generation of FLIR systems commonly includes a logic for dealing with these issues. Many systems of the past decade will automatically block any load transfer that would overload adjacent feeders. There is a clear need for more advanced FLIR systems that can divide the load into smaller 
pieces and can be supplied by multiple adjacent feeders without violating any voltage and capacity constraints and preferably in an optimal manner.

Some electric utilities have experienced problems with the FLIR systems that have operated successfully for years. Often these problems are the result of increased Distributed Generation (DG) penetration on the feeder. The fault contribution from the DG units can impact the existing protection and coordination of the system. As a result, the protection system might locate the fault incorrectly, and restoration switching activities may re-energize the fault and possibly lock out multiple feeders. This problem can be addressed by using "directional" fault location logic that identify the direction of current flow or by using centralized modeling approaches that account for short circuit contributions from distributed generators.

Another challenge resulting from the presence of distributed generators is what is referred to herein as the "net load" problem [5]. Present FLIR systems continuously monitor the power flowing into and out of each feeder section to determine the load that may need to be transferred if a fault event subsequently occurs. The problem arises when the DG unit trips off line when a fault occurs. Since the DG unit is not allowed to reconnect until approximately five minutes following restoration of normal primary voltage on the electric utility lines, the amount of load to transfer may exceed the amount measured just prior to the fault, potentially overloading the adjacent feeder. Also, the presence of microgrids on the feeders that need restoration of fault-free zones can cause similar issues. A possible solution is to monitor DG contributions at all times and account for the generation dropping off line when an outage occurs. But this solution requires extensive communication capabilities and may only be practical for larger, utility scale, DG units. In case of microgrids systems, they can stay islanded during the restoration process and help to bring additional segments of the system restored. One advantage with microgrid systems and utility scale DG units is that the required communication infrastructure would be already in-place as opposed to small DG units.

Volt/VAr control is another pressing issue in the context of FLIR systems as these systems need to be coordinated to ensure proposer operation of the system in normal as well as contingency conditions. Voltage regulation in traditional distribution grids is relatively simple and typically 
involves On-Load Tap Changers (OLTC), switched shunt capacitor banks, and line regulators acting on local control commands. However, due to increasing DG penetration in the distribution systems the DG units can contribute to voltage regulation at the Point of Common Coupling (PCC) and help mitigate the negative effects caused by its own penetration. The voltage regulation during the restoration process of the fault-free portions of the network needs to be coordinated and control parameters of various controllers or regulators may be updated subject to the completion of service restoration. The upcoming FLIR systems shall make use of dynamic voltage regulation support from Distributed Energy Resources (DER) and existing regulation equipment such as shunt capacitors and voltage regulators in the restoration logic.

The FLIR systems available today falls into two major categories, one that works based on centralized control approaches and the other with de-centralized/distributed control approach. Both the control strategies have their own merits and demerits. The centralized control approaches have the advantage of having access to all network data and are able to find the optimal solution. But, they tend to be inadequate since they are highly sensitive to system failures and should deal with large amounts of data which need powerful processing capabilities. Furthermore, since distribution systems are complex networks and constantly expanding, high communication capabilities are required in centralized control strategies which are costly.

Distributed control strategies can overcome some of the centralized approach shortcomings. In distributed control strategies there are more than one decision making units and the data processing can be done in parallel which makes it faster and requires less processing capabilities. Less communication capabilities are required in distributed control methods since there is no single central unit which is responsible for all communications. Multi-Agent Systems (MAS) are one of the most popular distributed control strategies which are used in power system applications because of their distributed nature and adaptability with dynamic environment of electric distribution systems. MAS is composed of a number of autonomous agents which work together to achieve a common goal.

Developing a business case that shows that the benefits accrued by the automated FLIR systems outweigh the costs is a daunting task. This is an important task in the economic feasibility process 
of Distribution Automation (DA) projects and one has to consider the trade-off between reliability and economics to arrive at the answer. FLIR functionalities on a distribution circuit require remote switching capabilities. The circuit to be automated is divided into zones by utilizing automated sectionalizing switches, so that the faulted area can be isolated from the fault-free portion of the network. Obviously, the more zones created, the more reliability improvements can be achieved due to confinement of fault to a smaller area, and more customers will be restored in fault-free sections of the feeder. However, investment costs also increase with the increase in number of zones and number of automated switches.

It makes the determination of optimal switch number and placement problem more challenging, and traditionally a trade-off between switch investments and reliability benefits are considered to solve the problem. Savings derived by the customers who experience fewer and shorter duration outages due to automated FLIR systems can be very significant. However, the use of customer outage cost savings is not accepted as cost justification in many jurisdictions in the US. Therefore, there is a need for a new economic approach to determine optimum number and placement of automated sectionalizing switches for DA projects.

\subsection{Approach}

A novel MAS DA design with agent learning capability is presented for electric distribution systems that works on a hybrid control philosophy to overcome the demerits of centralized and decentralized technologies. The proposed design addresses issues faced by the traditional FLIR systems due to increased penetration of distributed generation and microgrids. Moreover the proposed approach includes these components in the solution for improved FLIR performance during emergencies.

A new economic model is proposed based on relative reduction in the normalized customer interruption costs for the optimal switch number and placement problem. An iterative algorithm is constructed which minimizes the total interruption costs at each step of the analysis to arrive at the solution. The proposed approach isolates the impacts of varying switch investment and customer interruption costs that are usually based on outdated utility surveys. 
The MAS Fault Location and Isolation (FLI) approach accounts for fault characteristics of different types of DG resources that include synchronous, induction, and inverter-based resources. It is important to note that fault contribution from inverter-based resources is largely different from traditional rotating based resources, and it is imperative that FLI framework correctly model the fault behaviors of these resources for fault detection. Fault detection is achieved using the "Fault Detection Algorithm" in an autonomous fashion relying only on the local data measurements. Fault isolation activities are handled by coordinated communications among the agents.

Once the fault has been located and isolated, the service restoration takes place to restore power to as many un-faulted zones as possible while satisfying system constraints such as thermal loading and voltage. Service restoration problem is formulated as a combinatorial multi-objective optimization to maximize amount of load restored with minimal number of tie-switch operations while maximizing available microgrids in the system. Two algorithms, "Tie-Switch Ranking Algorithm" and "Zone Priority Algorithm", are introduced for service restoration tasks.

Although agents in MAS can solve the optimization techniques to find the near optimal configuration, it is also necessary that they learn new behaviors online, such that the performance of the agents and MAS gradually improves. Therefore, a learning methodology ( $Q$-Learning) with a modified reward function based on restoration performance is used to teach agents to use their experiences, and prevent them from doing the time consuming optimization process each time a fault happens.

The proposed MAS is designed to be demonstrated on Mon Power, a FirstEnergy company, distribution system as part of the DOE project, West Virginia Super Circuit (WVSC). Therefore, the proposed MAS DA design is tested through simulation studies of the actual distribution network of WVSC project. The distribution network is simulated using CYME $^{\circledR}$, Matlab $^{\circledR} /$ Simpower $^{\circledR}$, and PSCAD ${ }^{\circledR}$ software, whereas the MAS and its communications are simulated using Matlab ${ }^{\circledR}$ S-functions. 


\subsection{Contributions}

The main contributions of this work can be summarized as follows,

1. Hybrid MAS Architecture: A novel hybrid MAS architecture for FLIR is proposed that is flexible and scalable. The proposed MAS architecture includes dedicated agents for distributed generators and microgrids to include these components into the FLIR solution. The MAS framework is introduced in Chapter 4.

2. Switch Optimization: A new economic model is proposed for the optimal switch number and placement problem based on relative reduction in the normalized customer interruption costs. The iterative algorithm for switch optimization is discussed in Chapter 5.

3. Fault Location and Isolation: A distributed fault location and isolation approach is presented which uses the proposed "Fault Detection Algorithm" to determine the fault condition in an autonomous fashion relying only on the local data measurements. The proposed approach considers fault characteristics of different types of DG resources including synchronous, induction, and inverter-based resources. The fault isolation is achieved by coordinated communications among the agents. The proposed algorithm works for radial distribution system with any level of DG penetration. The FLI algorithm is described in Chapter 7.

4. Service Restoration: A new set of algorithms, "Tie-Switch Ranking Algorithm" and "Zone Priority Algorithm", are proposed to solve the service restoration problem efficiently. The problem is formulated as a combinatorial multi-objective optimization to maximize amount of load restored with minimal number of tie-switch operations while maximizing available microgrids in the system. The proposed approach accounts for and makes use of available distributed generation and microgrids in the restoration solution. The service restoration approach is discussed in Chapter 8. 
5. Reinforcement Agent Learning: A reinforcement agent learning algorithm based on $Q$ learning is developed to support agents with service restoration problem. Agents learn from their restoration experiences and use the learning knowledge for future restorations. Restoration problem formulation and learning approach are presented in Chapters 9.

\subsection{Dissertation Outline}

The outline of the remaining chapters is given in this chapter. In Chapter 2, we are giving a literature overview of the most significant research work in the area of FLIR and multi-agents system applications in electric distribution systems. The agent technology basics and agent communication standards are discussed in Chapter 3. Chapter 4 presents the proposed MAS architecture along with individual agent details in regards to their capabilities and embedded behaviors. It also presents the state transition mechanism the agents follow to achieve the organizational goals of FLIR, and the proposed FLIR process flow.

Determination of the optimum number and placement of automated switches for DA project is a complex task. Chapter 5 presents an innovative economic model based on relative reduction in the normalized customer interruption costs for the optimal switch number and placement problem. This chapter also presents switch optimization results for WVSC test system along with IEEE 34bus and 123-bus test feeders.

Chapter 6 presents fault characteristics and mathematical models of different DG resources including inverter based DG, synchronous generator, and induction generator. In Chapter 7 the proposed Fault Location and Isolation approach and the "Fault Detection Algorithm" are explained. The WVSC distribution network is presented which is used to demonstrate the proposed MAS FLIR solution. This chapter also presents detailed models developed for the WVSC distribution system using PSCAD software, and presents simulation results for different fault cases with varying levels of DG penetration.

In Chapter 8 the proposed MAS service restoration approach which includes "Tie-Switch Ranking Algorithm" and "Zone Priority Algorithm" is presented. The multi-objective optimization problem 
is discussed with defined objective functions and system constraints. This chapter also presents the Multi-agent logic to return to normal configuration, which is implemented after the fault condition is repaired. In Chapter 9 the basic idea of reinforcement learning based on $Q$-Learning method is explained and the modified $Q$-Learning approach for restoration decision making is described.

In Chapter 10, steady state modeling of the WVSC distribution system using CYME $^{\circledR}$ and Matlab $^{\circledR} /$ Simpower ${ }^{\circledR}$ software is presented. Simulation results for service restoration with the proposed optimization approach and Q-learning methodology are presented at the end of the chapter.

Finally, conclusions and future works are detailed in Chapter 11.

\subsection{Publications}

References $[6,7,8,9,10,11,12]$ are the works published or submitted from this research. 


\section{Chapter 2}

\section{Literature Review}

\subsection{Multi-Agent Systems}

A Multi-Agent System is a computational system in which several agents cooperate and coordinate with each other to achieve some objectives. Multi-Agent Systems have the capability to solve problems which are difficult or sometimes impossible to solve by an individual agent. Multi-Agent Systems are a branch of Distributed Artificial Intelligence (DAI). As a social community the agents are able to evolve, self-produce, learn, cooperate, and adapt. According to [13] the Multi-Agent System environment has the following characteristics,

- Multi-agent environment provides an infrastructure specifying communication and interaction protocols.

- Multi-agent environments are highly decentralized structures.

- Multi-agent environments contain agents that are autonomous and distributed, and may be self-interested and cooperative.

Research on Multi-Agent Systems is still an ongoing process. Although Multi-Agent Systems have shown good results, these technologies are yet to make a significant impact in the power system area. The authors in [14] have analyzed agent based technologies under the Strengths Weaknesses Opportunities Threat (SWOT) framework. Based on the SWOT analysis they have envisaged the 
future application of agents in the power system area. The following table shows the SWOT matrix developed by them.

Table 2-1: SWOT Matrix for Current Agent Applications to Power Systems [14]

\begin{tabular}{|c|c|}
\hline Strengths & Opportunities \\
\hline $\begin{array}{l}\text { - No single point of failure } \\
\text { - Decentralized data processing } \\
\text {-Fast operation } \\
\text {-Task distribution } \\
\text { - } \quad \text { Ease of tackling complex problems } \\
\text { - Natural solution } \\
\text { - Leads to scalable, modular, and flexible } \\
\text { solutions }\end{array}$ & $\begin{array}{l}\text { - Technological advances } \\
\text { - Collaboration/cooperation } \\
\text { - Develop generic agents/MAS } \\
\text { - Existing hardware or software } \\
\text { infrastructure } \\
\text { - Learn from other distributed } \\
\text { technologies }\end{array}$ \\
\hline Weaknesses & Threats \\
\hline $\begin{array}{l}\text { - Can lead to unstable operation } \\
\text { - Complexity } \\
\text { - Lack of understanding } \\
\text { - } \text { Lack of experts } \\
\text { - Lack in its in infancy } \\
\text { area }\end{array}$ & $\begin{array}{l}\text { - Unimagined problems } \\
\text { - Acceptability is not taken seriously } \\
\text { - Centralized schemes } \\
\text { - Security issues }\end{array}$ \\
\hline
\end{tabular}

Z. Zhang et al. in [15] identified and described three major problems of today's power system industry. They are distributed computing, communications, and data integration. They have suggested that the Multi-Agent System technology would be a best solution for the above problems in power systems because of the power system's distributed nature. They illustrated that the MAS can offer modular, flexible, and integrated approach for many problems in the power system area. They have proposed a four stage (Analysis, Design, Implementation, and Deployment) problem solving mechanism using Multi-Agent System.

\subsection{Switch Optimization}

Improvement of the system reliability is one of the main drivers of DA technologies. It is one of the most effective strategies in distribution networks to increase the reliability by reducing the duration of the interruptions and the number of affected customers. The key aspect of DA project is to install automated sectionalizing switches on the distribution feeders to divide them into 
multiple zones. The sectionalization of the feeder helps improving the reliability by isolating the faulted zone, and serving the un-faulted zones by adjacent feeders. The first step in any DA project's economic feasibility is the determination of number and placement of sectionalizing switches.

The problem of determining optimal number and placement switches has been studied by several authors with different approaches. Many works in literature formulated the problem as an optimization problem with objective functions such as reliability improvement and minimization of investment cost of switches. The reliability improvement is modeled as minimization of interruption costs. These methods are heavily reliant on the data pertaining to interruption costs and switch investment costs. The main problem with such data is the fact that it is not openly available, and even if it is available, seldom the data represent the present market conditions. Therefore, implementation of switch optimization problem using the actual values of interruption costs and investment costs might not always result in correct answer.

Authors in [16] proposed a Genetic Algorithm (GA) based approach to determine the best number of switches and their placement. A binary representation model and reliability measure SAIDI calculated from the annual non-supplied energy were used in the approach. Authors in [16] also proposed a solution to optimally allocate switches, reclosers, and fuses. The device allocation problem was modeled with non-linear integer programming and reliability index SAIFI was used as the objective function.

A Simulated Annealing approach is proposed in [17] which considered the investment, maintenance, and outage costs in a single global cost function to determine the optimal number and location of switches. A decomposition approach is presented in [18] and the solution is represented as a binary array of the possible locations for sectionalizing switches. The problem complexity was reduced by using a polynomial-time partitioning algorithm to decompose the problem into a set of convex independent sub-problems to be solved independently.

Authors in [19] proposed an immune algorithm to solve the problem of optimal allocation of switches. The solution was to minimize the objective function which is sum of the customer 
interruption cost and the investment cost of installing switches. The immune algorithm based solution was used to design the DA system for a real distribution system in Taiwan Power Company. The work in [20] uses a reactive tabu search to find the optimal device allocation. The objective function was a sum of estimated interruption costs.

Authors in [21] proposed the solution based on cost/worth approach and the best locations of switches are determined by Simulated Annealing algorithm. A trade off analysis between sectionalizing switch cost to the reliability worth was used in the solution. A Particle Swarm optimization approach was proposed in [22] to determine the optimum number and locations of two types of switches, sectionalizer and breakers, in radial distribution systems. Authors in [23] proposed an Ant Colony optimization based method using fuzzy multi-objective formulation for finding the number and location of sectionalizing switches in distribution networks including DG.

\subsection{Fault Location and Isolation}

Fault location in distribution systems is an important function for outage management and service restoration. It directly impacts feeder reliability and quality of the electricity supply for the customer. Fault location is a more challenging task in distribution systems than transmission systems. This is due to the nature of the distribution systems such as heterogeneity of the lines, presence of laterals, load taps, presence of distributed generation, and comparatively a lower degree of instrumentation [24]. The methods aimed at transmission level usually use data from Power Quality (PQ) monitors and protection devices. At distribution level, the data is usually more limited with inadequate communication infrastructure.

The existing fault location methods in the literature can be mainly classified into three categories:

1. Impedance based methods

2. Wavelet based methods

3. Intelligent methods such as Artificial Neural Networks (ANNs), Expert Systems, and MAS. 
Impedance based methods usually calculate the apparent impedance using the voltage and current phasor measurements from field devices. The apparent impedance is used to estimate the potential fault locations in the system based on specific algorithms. These methods can successfully locate the faults for distribution systems that have fairly simple structures without many lateral taps. The common drawback is that the fault location results in multiple fault location estimations due to their reliance solely on measured voltage and current signals at the substation. A detailed review of the existing impedance methods is presented in [25].

Recently some methods have been developed and adopted by utilities for fault location based on the Impedance approach. In [26], an approach is introduced to compute apparent reactance by using fundamental voltage and current phasors and estimated fault location based on the computed reactance. At distribution level, the performance of this method suffers due to load effects. In [27], Santoso et al. utilized load changes to identify the line sections where the fault may have occurred. In [28], a current and impedance change method has been adopted to estimate the location of the fault in the distribution system with distributed generation.

In [29] [12] the authors used the data from PQ monitors and compared the data with simulation data for better fault location. In [30], Saha et al. adopts a similar approach and proposes to use the data from the existing microprocessor relays. To overcome the line model dependency problem, in [31] an ANN based estimator was proposed to estimate fault locations. In [32], after the candidate sections are found, the feeder topologies and load current change were considered to further rank candidate fault locations.

Some fault location methods have been developed that uses wavelet analysis of fault current waveforms using Fourier Transform. These methods are less reliable because of variety of load characteristics and fault causes in distribution systems. Authors in [33] introduced a wavelet based method for the detection of High-Impedance Ground Faults (HIF) in distribution systems. The ground faults are detected by the estimation of phase displacements between zero-sequence voltage currents in the feeders. It is shown that the analysis of transients in zero-sequence currents and voltages in wavelet domain leads to fast and reliable HIF detection and localization of the 
faulted feeder. The proposed algorithm remains stable for any network changes, and is independent of the network neutral-point grounding mode.

Fault location methods based on intelligent systems such as neural networks and fuzzy logic are proposed in [34], [35]. The major limitation of intelligent methods with learning is the fact that they require retraining with huge amount of data sets subsequent to every change in system topology. In [34], faulted area is detected by training an Adaptive Neuro-Fuzzy Inference System (ANFIS) net with extracted features based on knowledge about protective device settings. In [35] using the Learning Algorithm for Multivariable Data Analysis (LAMDA) classification technique, multiple fault location estimation solution is obviated.

\subsection{Service Restoration}

The service restoration is a combinatorial problem since it involves many combinations of switching operations to restore power to customers during emergencies. Presently, service restoration problem is solved using various approaches such as Heuristics [36], Expert Systems [37], Meta-Heuristics [38, 39, 40], and Mathematical Programming [41] [42]. Some of the MetaHeuristic techniques like Genetic Algorithm (GA), Particle Swarm Optimization (PSO), Simulated Annealing (SA), and Tabu Search (TS) have been used extensively for the restoration problem. Artificial Neural Network (ANN), Dynamic Programming, and Ant Immune System - Ant Colony Optimization (AIS-ACO) are also studied for restoration problem.

A key disadvantage of most of the techniques for service restoration problem mentioned above is the fact that they all work in a centralized fashion to solve the problem. All of these techniques require a powerful central computing facility with high communication requirements to haul huge amounts of data with minimal latency. This dependency can lead to a single-point-of-failure defeating the key purpose of DA systems to improve system reliability. 


\subsubsection{Expert Systems and Heuristics}

Expert Systems and Heuristic methods use some rule-based programming logic based on operator's knowledge and experience. This reduces the search space of the combinatorial restoration problem and the solution is achieved in less time with minimal computational burden. The main drawbacks of Heuristic methods are, optimal solution is not guaranteed, and the software that handles the rule-set can become complex as the system size grows. Morelato and Monticelli [36] have presented a Heuristic search approach for distribution system restoration which uses a knowledge guided search strategy to reach the solution. Liu et al. in [37] designed an Expert System algorithm for restoration and loss reduction of distribution systems. The authors constructed a knowledge base which contains rules that implement a solution approach that system operators can use for service restoration.

\subsubsection{Mathematical Programming}

The Mathematical Programming approach determines the target configuration for restoration often by formulating the problem as a Mixed Integer Non-Linear Problem (MINLP). In the formulation each switch status is denoted with a binary value " 1 " if the switch status is close and " 0 " if the switch status is open. Other parameters such as voltage and thermal capacity are modeled as continuous variables to specify the constraints for the optimization problem. The mathematical programming can find optimal target reconfiguration, however, the main drawback of this technique is computational intensity and it might not be a practical solution for large distribution systems.

Nagata et al. in [43] proposed a two-stage algorithm which decomposes the restoration problem into two sub-problems, one to maximize available power to the de-energized area, and two to minimize the amount of unserved energy. The algorithm was tested on dc models which does not take into account the reactive power as well as voltage variations. Authors in [42], presented a Dynamic Programming approach with state reduction for service restoration in radial distribution system. The timing and selection of feeders to be energized are represented as states in a Dynamic Programming formulation. The proposed Dynamic Programming method reduces the number of 
states by grouping states that are close to each other and selecting the best state. S. Das et al. in [41] presented a restoration methodology based on reinforcement learning for ship-board power system. The proposed approach not only determines the optimal target configuration but also the correct order in which the switches are to be changed.

\subsubsection{Multi-Agent System technology}

Multi-Agent System technology emerges as an application of distributed intelligence that deals with concentrating the intelligence on a component level. MAS is a computational system in which several agents cooperate and coordinate with each other to achieve organizational objectives in a decentralized fashion [44]. This technology is superior to centralized schemes mentioned above as even though a part of the system fails rest of the system still continues to work. MAS can be considered as the platform of distributed processing, parallel operation, and autonomous solving. Further, it can be much faster in solving discrete and nonlinear problems. Therefore, MAS as a distributed restoration algorithm represents an interesting candidate for restoration and control of the distribution system to realize the self-healing operation. In the past, several authors have worked on MAS technology for solving service restoration problem in power systems.

J. M. Solanki et al. in [45] introduced a totally decentralized MAS solution for the service restoration in distribution systems. The Multi-agent platform is developed in Java Agent Development Framework (JADE) and the power system test case is developed in Virtual Test Bed (VTB). In their work they proposed three new agents named Switch Agents (SAs), Load Agents (LAs), and Generator Agents (GAs). The agents communicate only to their neighboring agents and act locally, making the restoration scheme decentralized. The restoration algorithm has certain objectives and constraints like, limit on generation, priority of loads, and transfer capacity of lines.

Nagata et al. in [46, 47] have proposed a Multi-agent architecture for power system restoration problem. This architecture was fully decentralized one, in which the agents communicate and negotiate only with their neighboring agents. In this work authors have used two types of agents named Bus Agents (BAGs) and Facilitator Agent (FAG). BAG is a bus agent situated at each and every bus in the network, whereas, FAG is a Facilitator Agent situated on top of all BAGs for the 
decision making purpose. BAGs communicate with their neighboring agents to find out the suboptimal target configurations, whereas, FAG acts as a manager for decision process. Knowledge Query and Manipulation Language (KQML) is used as an agent communication language.

In [48], authors have proposed a more complicated four layered agent architecture for the bulk power system restoration. A single Independent System Operator Agent (ISOAG) which controls the timing of the simulation process forms the higher level in the agent architecture. The second level consist several Local Area Management Agents (LAMs) for the local area management of the restoration. Third level of architecture has several Load Facilitator Agents (LFAGs), Generator Facilitator Agents (GFAGs), and Remote Facilitator Agents (RFAGs). These agents will facilitate with the agents situated in the fourth layer of architecture, which include Load Agents (LAGs) and Generator agents (GAGs). The authors have applied the proposed MAS to bulk power system and the simulation results are very promising.

Authors in [49] have also contributed in the field of Multi-Agent System for bulk power system restoration. The method employed in this work makes use of both centralized and decentralized schemes to obtain the restoration solution. The agent architecture has two hierarchical agents called, Management Agents (MGAGs) and agents representing practical power system components (PCAGs). Each PCAG monitors several agents related to a subsystem named Generator Agents (GAGs), Substation Agent (SAGs), and Load Agents (LAGs). One MGAG is generated in charge of the subsystem and provides negotiations and communications between PCAGs and with other subsystems.

Authors in [6] proposed a hybrid MAS solution for fault detection and service restoration problem. The proposed approach in [6] reconfigures the system in a way to supply all the critical loads. When there are multiple solutions available for reconfiguration, the one with good voltage profile and less power loss is selected as the solution. The proposed algorithm makes use of shunt compensation and priority based load shedding in order to control the voltage across the network. Authors in [50] have used artificial immune system algorithm for the fault restoration problem. In [51] the author demonstrated a practical implementation of MAS for FLIR in which agents are implemented in microprocessors and the distribution system is simulated using a power systems 
simulator. Authors in [52] have designed a hierarchical MAS architecture for DA system to locate the faulted zone and restore power to un-faulted zones with the help of agent learning.

\subsection{Agent Learning}

Multi-Agent Systems are rapidly finding variety of applications in power system domain. The size and complexity of problems in power system make difficult to solve them with pre-programmed agent behaviors. The MAS solutions often require time consuming agent interactions and communications to achieve the desired goals. Therefore, agents must learn from their experiences to discover solutions autonomously within shortest possible time. Although the agents in MAS can be programmed with behaviors designed in advance, it is also necessary that they learn new behaviors online, such that the performance of the agents and MAS gradually improves [13], [53].

The concept of reinforcement learning originated from Thorndike's research on animals in 1911 [54]. The research established that animals could learn to associate their actions with the rewards through trial and errors. The first formalization of reinforcement learning happened in the learning automata model in 1970s [55]. In early 1980s, Sutton and Barto [54] developed temporaldifference learning, which is another form of reinforcement learning. Further attention was drawn to reinforcement learning after Watkins and Dayan proposed Q-learning in 1992 [56], which established the connection between reinforcement learning and Markov Decision Processes (MDP).

A $Q$-learning agent learns by interaction with dynamic power system environment without the need of a teacher. At each time step, agents perceive the complete state of the system and take an action, which causes the environment to transition into a new state. Every such transaction will result in a scalar reward indicating the quality of transaction. The agents learn from quality of the transactions, and as time passes the agent learning can converge. $Q$-learning is an online approach, which not only let agents tune themselves with time but also re-adapt to the non-stationary environment. This makes $Q$-learning less susceptible to system drift due to equipment aging, replacements, and etc. [41]. 
In this dissertation, a MAS concept for self-healing power distribution grids with agent learning capability is proposed. The main note-worthy contribution of this research is the modified $Q$ Learning approach to solve service restoration problem in power distribution systems with distributed energy resources. The learning methodology is used to teach agents to use their experiences and update their knowledge as the system is operational. The $Q$-Learning technique introduced in this dissertation uses $Q$-matrix and switch combination matrix as the repositories for all the information learned through previous restoration experiences. Agents can utilize the learning for service restoration instead of performing time consuming optimization, if there is sufficient learning from restoration experiences.

$Q$-learning has been extensively used to solve the strategy control and learning problems of autonomous agents. Sanjoy Das et al. in [41] used $Q$-Learning for dynamic reconfiguration of shipboard power systems. The problem of service restoration is formulated as an optimization problem with an objective to maximize the weighted sum of the power delivered to the loads. The prosed approach is a dynamic technique that produces not only the final reconfiguration, but also the correct order in which the switches are to be changed. In [57] $Q$-Learning is used for power system restoration following catastrophic disturbances involving loss of generators. The proposed $Q$-learning restorative framework supports agents to make accurate decision in a timely manner to restore the system without requiring a global reward signal. Jung et al. in [58] presented a Multiagent framework to avoid catastrophic failures of a power system by introducing an adaptive agent learning method for load shedding based on Temporal Difference (TD) method, which is one of the forms of Reinforcement Learning (RL).

\subsection{Existing FLIR Systems}

Until the 1990s, technological breakthroughs in the automation of electric distribution systems were slow to materialize. Early SCADA systems promised to improve reliability. But many of the early systems were little more than an extension of SCADA beyond the substation fence. With their relatively low computational and communication capabilities, these early systems required a high degree of human intervention and offered little in the way of true automation. As a result, expectations were not always met, leading to the termination or scaling back of many projects. 
Nevertheless, many lessons were learned and technology has advanced, especially through the 1990s and to the present. More advanced automation applications have been developed that are far more practical and manageable for utilities to implement. Some tools for feeder automation have been available for many years. Below we presented the S\&C Electric Company product (IntelliTEAM-SG) which is a patented and commercialized product and compare it to the proposed FLIR system presented in this dissertation [4, 59].

\subsubsection{IntelliTeam SG}

In 2003, the IntelliTEAM Automatic Restoration System was introduced by S\&C Electric Company. IntelliTEAM II is a patented automatic power restoration system that uses distributed intelligence and peer-to-peer communication to switch and isolate the faulted line section and restore power to un-faulted line sections.

IntelliTEAM utilizes ties to multiple circuits, enabling a wide variety of circuit configurations to maximize the restoration potential. Distributed intelligence prevents line overloads. IntelliTEAM can automate parts of a circuit in need of attention or an entire distribution system consisting of open-loop feeders with multiple tie points to other feeders. It can be used both tactically and strategically, based on the reliability problems the utility needs to solve.

\subsubsection{IntelliTeam Working Principle}

IntelliTEAM dynamically tracks system conditions on overhead and underground distribution systems and provides fully automatic fault isolation and service restoration. "Team" and "Coach" metaphors help to describe IntelliTEAM operation. Each team is defined as a line segment bounded by two to eight automated switches, each of which can represent a different power source. Each team has a software coach. If an outage or line fault occurs, local control logic determines if a switch should open. The coach of each affected team then uses real-time data, including voltage and current data gathered prior to the system event to develop a restoration strategy for un-faulted sections. The coaches of adjacent teams then work together through shared controls to implement strategies that will maximize restoration of the circuit within the prioritization rules defined by the user. 
Breakers and reclosers can also be incorporated as team members with the addition of a module that interfaces to the electronic relay or control that operates the breaker or recloser. Figure 2-1 illustrates the team concept. The coach distributes data and coordinates the operation of automated switches, reclosers, and breakers. Coach travels to each team member within a prescribed time period. Coaches are responsible for restoration decision makings and they move to each switch that has the capability for restoration and ask the neighboring team coach to move to the same switch to make restoration decisions. When a coach moves to a team member to investigate the restoration possibility, it searches for the neighboring coach from the adjacent teams.

In the event of a loss of communication, service restoration may be inhibited, but an automated switch, recloser, or breaker can still isolate a fault based on local fault detection and isolation software logic.

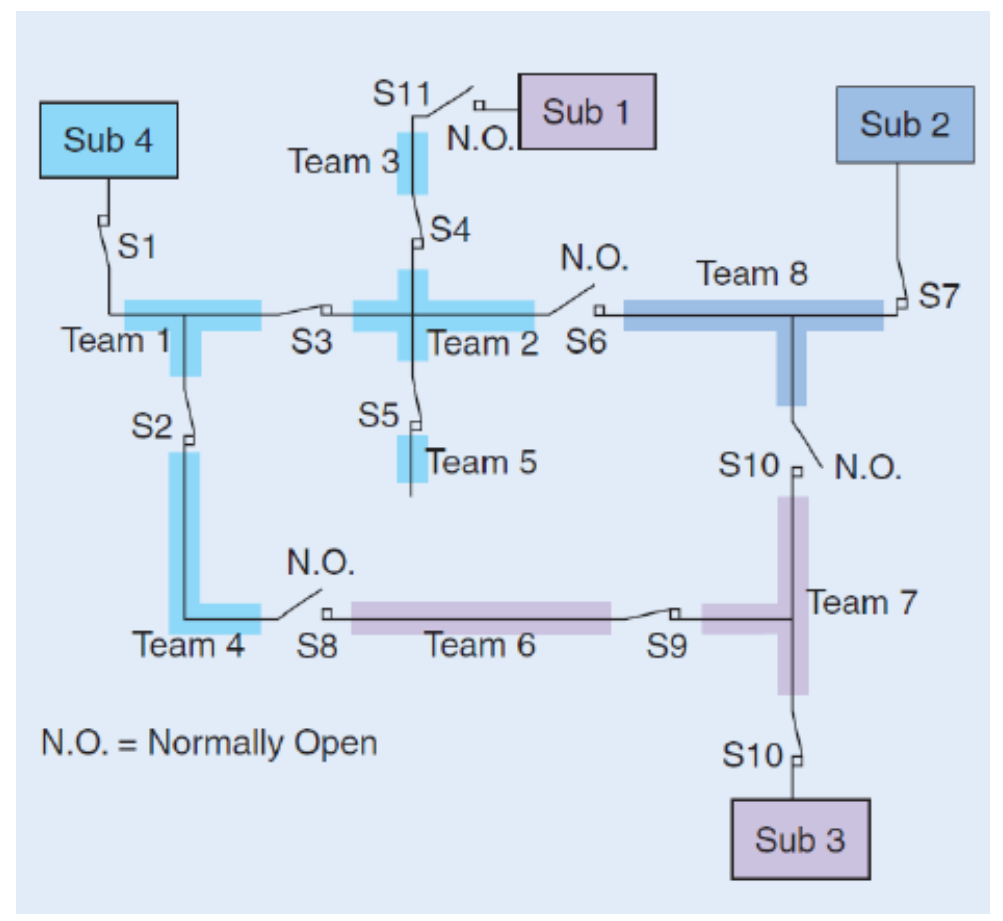

Figure 2-1: An Example of IntelliTEAM Implementation

At the beginning of a fault or outage event, any team member detecting the event will communicate to all the other team members and the coach, reporting the time, type of event, report identification, 
and so on. The coach also monitors the status of each device, ensuring that all are in the right open or closed position to allow progress to the next restoration step.

In order to close a switch, the coaches of adjacent teams must grant permission based on circuit conditions, location of fault, and an evaluation of loading conditions. When all appropriate conditions are met, the switch can be closed to restore service to a feeder segment. This process is repeated for each segment in turn. As switches close, the IntelliTEAM system updates available capacity on the feeders used to restore service, ensuring maximum service restoration even at maximum peak load.

Following a reconfiguration event, and subsequent repair and restoration of the faulted line section, team members can return to their normal state. Once the "return to normal" is initiated, the coaches begin the process of returning each team to its normal configuration. The return-to-normal process begins at the team closest to the normal source and works outward, using either an open or closed transition approach as defined by the user.

To support the distributed control architecture of IntelliTEAM, a peer-to-peer wide-area communication network is essential. There are only a few commercially available wide-area communication systems that readily support such a network. Typically, communication for the IntelliTEAM system is provided by $900-\mathrm{MHz}$ UtiliNet spread- spectrum radios. The UtiliNet radio system transports data using a connectionless mesh architecture. This architecture allows each radio in the network to act as both a repeater radio and a data transceiver for an automated switch. The radio network is highly scalable and obviates the need to invest in the tall master radio towers prevalent in point-to multi point radio networks.

Fiber-optic networks have also been utilized for high speed peer-to-peer communication, typically in underground applications where fiber is installed along with the underground cable infrastructure. 


\subsubsection{FLIR \& IntelliTeam}

In IntelliTEAM the local control logic determines if a switch should open after a loss of voltage, and/or fault current event. A team member would open to isolate the fault after sensing the fault currents and two times loss of voltage (because of recloser operations). Also, if a switch does not sense the fault current it opens after two times loss of voltage. The fault location can be determined based on the location of switches that sensed the fault current. This FLI method has the following disadvantages:

- It does not support the DG penetration: In case there are DG's in the power system and two way power ow exists, proposed FLI system might not be able to locate the fault.

- It requires threshold settings for fault currents which is challenging and should be modified after any change in the system configuration.

For restoration, the coach of each affected team uses the real-time data, plus voltage and current data gathered prior to the system event, to develop a restoration strategy for un-faulted sections. The coaches work together to implement strategies that will maximize restoration within the prioritization rules defined by the user. A coach moves from the switch to switch based on the predefined source priorities and checks the restoration possibility through that switch and by negotiating with the neighboring coaches. 


\section{Chapter 3}

\section{Multi-Agent Systems}

Agent based technologies have been the subject of extensive research and investigation for several years, but it is recently that MAS have been seen significant degree of exploitation in commercial applications. Multi-Agent Systems have been used in vivid applications ranging from small systems to most intrinsic systems. Some of the fields where these Multi-Agent Systems have been fruitfully employed include process control, system diagnostics, manufacturing, transportation logistics, and network management [44].

Before understanding the Multi-Agent Systems, one has to understand the concepts behind the single agent. Many people have defined the word "agent" in their own ways since the starting of agent technologies. Out of many definitions available a couple of relevant definitions are given below,

"An agent is an autonomous computational entity such as a software program that can be viewed as perceiving its environment through sensors and acting upon this environment through its effectors" - Weiss [13].

"An agent is a computer system that is situated in some environment, and that is capable of autonomous action in this environment in order to meet its design objectives" - Wooldridge and Jennings [60]. 
The above two definitions are much suitable for this dissertation work. Multi-Agent Systems received greater attention in the recent past in many intelligent and distributed systems to model the theories of interactivity in human societies. Humans interact in various ways and at different levels. For an instance, humans observe and model one another, they request and provide information, they negotiate and discuss, they develop shared views of their environment, they detect and resolve conflicts, and they form organizational structures such as teams, committees, and economies.

In this dissertation the term agent means, a software entity which perceives its environmental changes and acts on it diligently to achieve its organizational objectives. A Multi-Agent System means the collection of such agents which communicate and coordinate to solve a particular problem according to their objectives. The Multi-Agent System approach for electric distribution system is shown in Figure 3-1,

\section{Electric Distribution System (EDS)}

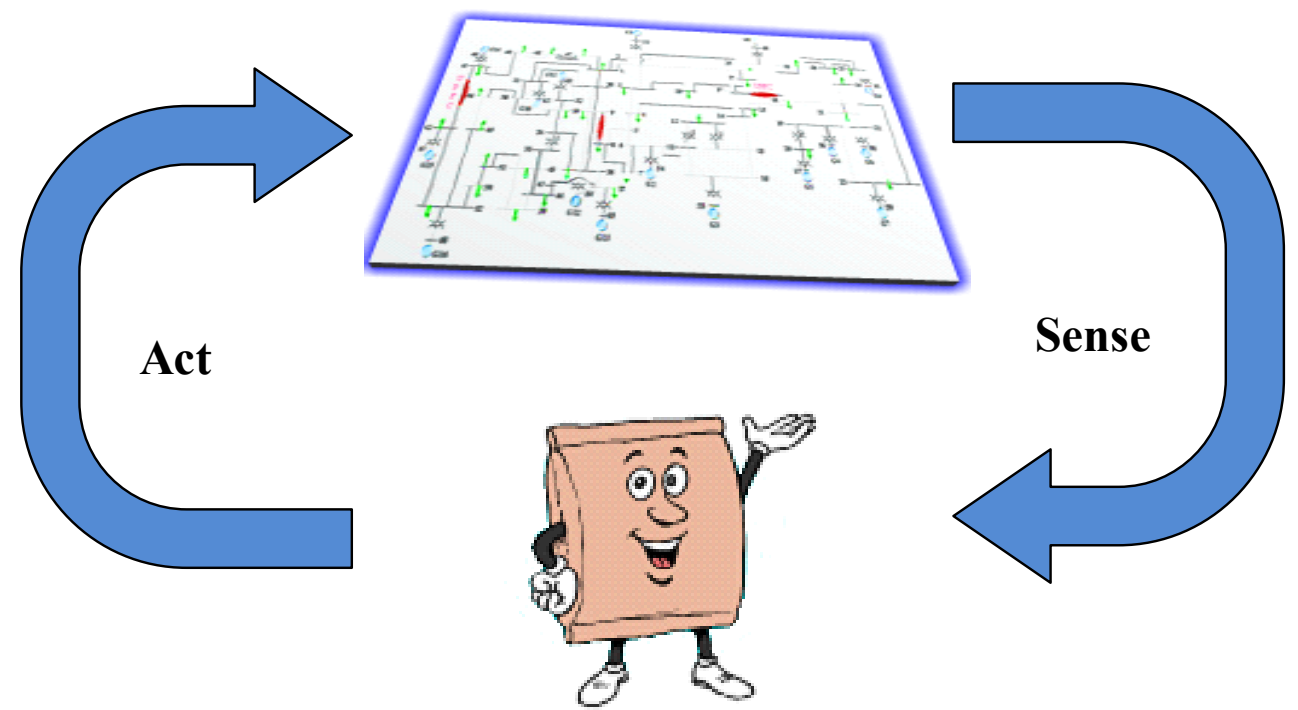

Agent

Figure 3-1: Multi-Agent System for Electric Distribution System

The main problem facing by an agent is deciding which of its actions it should perform in order to best satisfy its organizational objectives. The type of decision selected always depends upon the 
type of environment. Russell and Norvig [61] proposed following classification of environment properties,

\section{- Accessible vs Inaccessible.}

An accessible environment is one in which all the states of the environment are easily accessible. But, most of today's complex networks, such as every day physical world and Internet, are inaccessible.

\section{- Deterministic vs Non-deterministic}

In deterministic environment the actions will have certain effects on the environment. In non-deterministic environments it is difficult to anticipate the effects of particular action on the environment. Non-deterministic environments will have greater difficulty in designing.

\section{- Episodic vs Non-episodic}

In an episodic environment, the performance of an agent is dependent on a number of episodes, such as mail sorting system.

\section{- Static vs Dynamic}

A static environment is one that can be assumed to be unchanged except for the actions by agent. In contrast to the static environment the real world systems are dynamic systems which change from time to time, hence, difficult to control.

\section{- Discrete vs Continuous}

An environment is said to be discrete, if it has discrete number of actions and percepts in it. The best example for a discrete environment is the chess game and for a continuous environment is car driving.

Comparing the agents to the present world computer systems, every action a computer performs is anticipated, planned for, and coded by a programmer. When a computer encounters a new sort of problem, which is not anticipated, then the system will crash. This is because the computer systems do not have intelligence embedded in it. Unlike the computer systems the agents are intelligent enough to take up the flexible autonomous actions to satisfy their objectives [60]. Here the flexibility means three things, 


\section{- Reactivity:}

Agents are able to perceive their environment, and respond in a timely fashion to changes that occur in it, in order to satisfy their objectives.

\section{- Pro-activeness:}

Intelligent agents are able to exhibit goal oriented behavior by taking the initiative in order to satisfy their objectives.

\section{- Social-ability:}

Intelligent agents are able to communicate and cooperate with other agents available in Multi-Agent Systems in order to satisfy their objectives.

Out of the above three characteristics the social-ability is the more complex one. In real world daily millions of computers exchange the information with both humans and other computers. But, the ability to exchange the bit stream is not really the social ability. Consider the human world where humans achieve their goals by negotiation and cooperation [13]. The same way intelligent agents cooperate and negotiate with each other to achieve the common goal of the Multi-Agent System. The communication between the agents in MAS has certain standards defined by Foundation for Intelligent Physical Agents (FIPA).

There is always been a great amount confusion between agents, objects, and expert systems. The main distinctions between traditional view of an object and an agent is listed below [13],

- Agent embody a strong notion of autonomy than objects, they decide on themselves whether or not to perform an action on request from other agents.

- Agents are capable of flexible (reactive, pro-active, social) behavior. Where, objects have no such behaviors.

- A MAS is inherently multi-threaded where each agent has at least one thread of control.

Expert Systems are the most important Artificial Intelligence (AI) technology of 1980s [62]. An Expert System is one that is capable of solving problems or giving advice in some knowledge rich domain [13] [63]. The main difference between Expert Systems and Agent technologies is that, an Expert System cannot sense its environment directly. Instead, it needs a middle man to input the 
data. In the same way an Expert System cannot act directly on its environment. It just gives certain instructions to the operator.

\subsection{Agent Communication}

It has been seen in the earlier section that agents communicate to coordinate and negotiate on particular tasks to achieve its organizational objectives. The communication ability of agents is part perception (the receiving of messages) and part action (the sending of messages). There are generally two message types in agent communication, Assertion and Queries. Every agent whether active or passive, must have the capability to accept the information. In order to assume a passive role in a dialogue, an agent must be able to 1) accept a query from an external source and 2) send a reply to the source by making an assertion. In order to assume an active role in a dialogue, an agent must have the capability to send queries and make assertions [13].

Communication protocols are very important in any communication systems. The Multi-agent communication is specified by a data structure with the following five fields,

- Sender.

- Receiver(s).

- Language in protocol.

- Encoding and Decoding functions.

- Action to be taken by the receiver(s).

Speech Act Theory helps in defining the message send by sender. So, the receiver has no doubts regarding the received message [64]. In order to understand communication messages precisely, all the agents in MAS has to follow the same ontology. Two agents following the same ontology means, those two agents know the meaning of messages during the communication. Hence, ontology results in perfect operation of communication. In technical terms the name ontology is defined as follows, 
"Ontology is a formal definition of a body of knowledge. The most typical type of ontology used in building agents involves a structural component. Essentially a taxonomy of class and subclass relations coupled with definitions of the relationships between these things" - Jim Hendler [13]

The most common languages followed for the agent communication are,

- Agent Communication Language (ACL)

- Knowledge Query and Manipulation Language (KQML)

\subsubsection{FIPA-ACL}

Foundation for Intelligent Physical Agents (FIPA) is an IEEE Computer Society Standards organization that promotes agent-based technology and the interoperability of its standards with other technologies. FIPA was originally formed as a Swiss based organization in 1996 to produce software standards for heterogeneous and interacting agents and agent based systems [65].

FIPA has developed a certain number of standards for heterogeneous agent communications. FIPA Agent Communication specifications deal with Agent Communication Language messages, message exchange interaction protocols, speech act theory based communicative acts, and content language representations.

Agent Communication Language developed by FIPA is based on speech act theory. Here the messages are actions or communicative acts, as they are intended to perform some action by virtue of being sent. The FIPA ACL specifications consist of a set of message types and the description of their pragmatics, which is the effect on the mental attitudes of sender and receiver agents. A sample FIPA ACL message is shown below, 


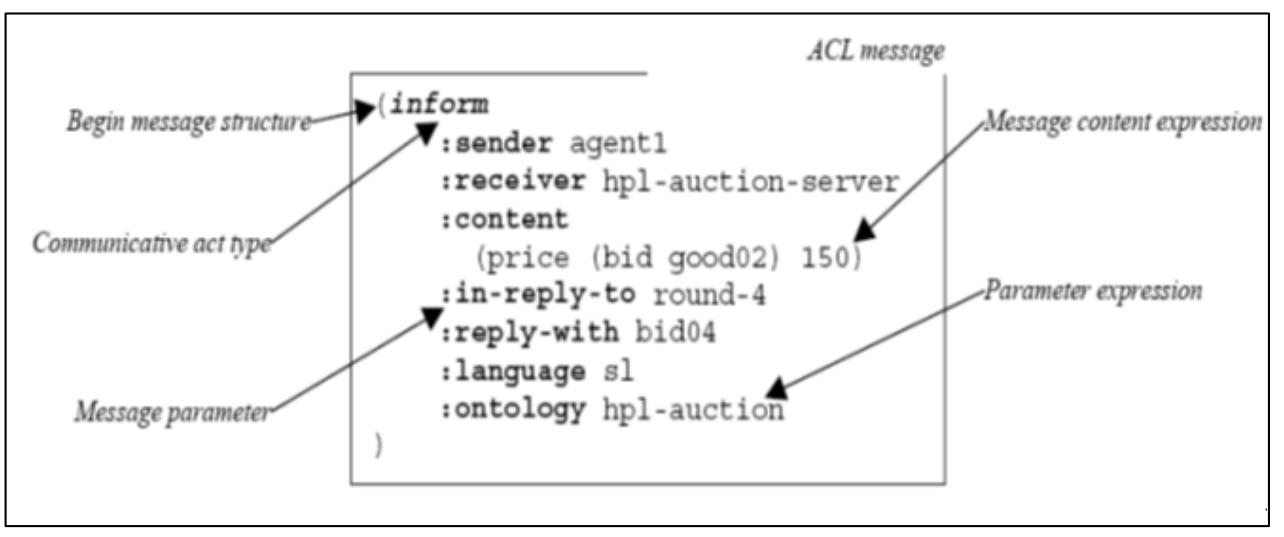

Figure 3-2: FIPA ACL Message [66]

As noted above, the ACL message contains many parameters. These parameters can occur in any order in the message. The only parameter which is mandatory for the agent communication is :performative parameter. Performative parameter indicates type of the communication act, and some typical performatives include Query_ref, Request, Accept, Inform, and Refuse. However, for a meaningful message there should be other parameters required depending upon the situation. The full set of pre-defined message parameters is shown in the following table.

Table 3-1: FIPA ACL Message Parameters [66]

\begin{tabular}{|c|c|}
\hline $\begin{array}{l}\text { Message } \\
\text { Parameter }\end{array}$ & Meaning: \\
\hline :performative & Type of communication act \\
\hline :sender & Denotes the identity of the sender of the message. i.e., the name of the agent. \\
\hline :receiver & Denotes the identity of the intended recipient of the message. \\
\hline :content & Denotes content of the message; equivalently denotes the object of the action. \\
\hline :reply-with & $\begin{array}{l}\text { An expression which will be used by the agent responding to this message to } \\
\text { identify the original message. Can be used to follow a Conversation thread in a } \\
\text { situation where multiple dialogues occur simultaneously. }\end{array}$ \\
\hline :in-reply-to & $\begin{array}{l}\text { Denotes an expression that references an earlier action to which this message is } \\
\text { a reply. }\end{array}$ \\
\hline :envelope & $\begin{array}{l}\text { Denotes an expression that provides useful information about the message as } \\
\text { seen by the message transport service. The content of this parameter is not } \\
\text { defined in the specification, but may include time sent, time received, route, etc. }\end{array}$ \\
\hline :language & Denotes the encoding scheme of the content of the action. \\
\hline :ontology & $\begin{array}{l}\text { Denotes the ontology which is used to give a meaning to the symbols in the } \\
\text { content expression. }\end{array}$ \\
\hline :reply-by & $\begin{array}{l}\text { Denotes a time and/or date expression which indicates a guideline on the latest } \\
\text { time by which the sending agent would like a reply. }\end{array}$ \\
\hline
\end{tabular}




\begin{tabular}{|l|l|}
\hline :protocol & $\begin{array}{l}\text { Introduces an identifier which denotes the protocol which the sending agent is } \\
\text { employing. The protocol serves to give additional context for the interpretation } \\
\text { of the message. }\end{array}$ \\
\hline :conversation-id & $\begin{array}{l}\text { Introduces an expression which is used to identify an ongoing sequence of } \\
\text { communicative acts which together form a conversation. A Conversation may } \\
\text { be used by an agent to manage its communication strategies and activities. }\end{array}$ \\
\hline
\end{tabular}

The Java Agent Development Environment (JADE) developed by Telecom-Italia makes use of ACL protocol for agent communication.

\subsubsection{KQML}

Knowledge Query and Manipulation Language (KQML) is older than the FIPA ACL language. KQML is a protocol for exchanging information and knowledge. The advantage of KQML language is that, all information for understanding the content of the message is included in the message pattern itself [13]. The basic protocol for the KQML language is defined by the following structure,

(KQML-performative

$$
\begin{array}{ll}
\text { : sender } & <\text { word }> \\
\text { : receiver } & <\text { word }> \\
\text { : language } & <\text { word }> \\
\text { : ontology } & <\text { word }> \\
\text { : content } & <\text { expression }> \\
\ldots) &
\end{array}
$$

The KQML protocol for communications between agents and application program is illustrated in Figure 3-3, 


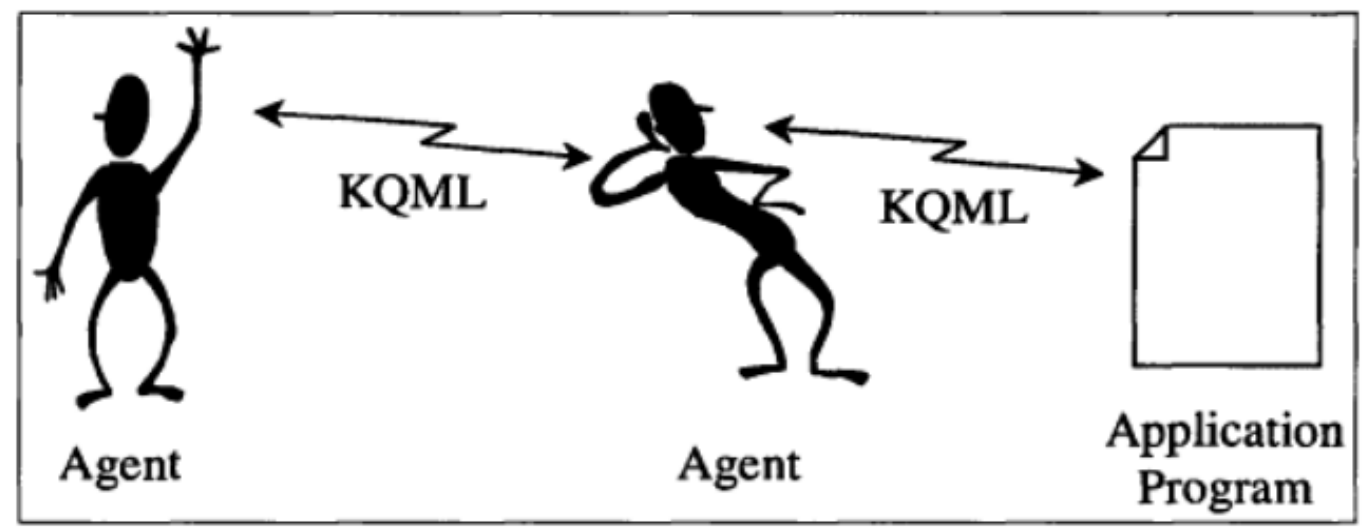

Figure 3-3: KQML Communication Protocol [13]

Most of today's agent based platforms have migrated to FIPA ACL language for the agent communications. 


\section{Chapter 4}

\section{MAS FLIR System Design}

\subsection{Proposed MAS Architecture}

The proposed hybrid MAS has five types of agents namely Substation Agent (SA), Feeder Agent (FA), SSW Agent (SSA), TSW Agent (TSA), and PCC Agent (PA) distributed in three hierarchical layers as shown in Figure 4-1. As the name indicates these agents are situated at the respective hardware points in the real field. The total number of agents for a self-healing FLIR system depends on total number of substations, feeder reclosers/breakers, SSW, TSW, and PCC switches present in the targeted distribution system.

Each layer has a set of agents that can communicate with other agents in the same layer and with the agents that they are associated in the downward layer. FA can communicate with its neighboring FAs in the same layer, and with its children SSAs, TSAs, and PAs in the downward layer. This makes the MAS architecture hierarchical. However, the supervisory agents, FA and SA, also exploit peer to peer communications with the lower level switch agents to reduce number of communications to speed up the FLIR processes. The decision making happens at the supervisory level, at the FA or SA level, as these agents have the complete view of the system to achieve the optimal solution.

Each agent knows limited local information and if it needs information outside its boundaries it communicates with respective agents to gather that information. For e.g., if a SSA wants to know about the available feed capacity of a particular TSA, then it communicates with that respective 
TSA to gather the required information. This arrangement makes the MAS architecture to be modular and decentralized.

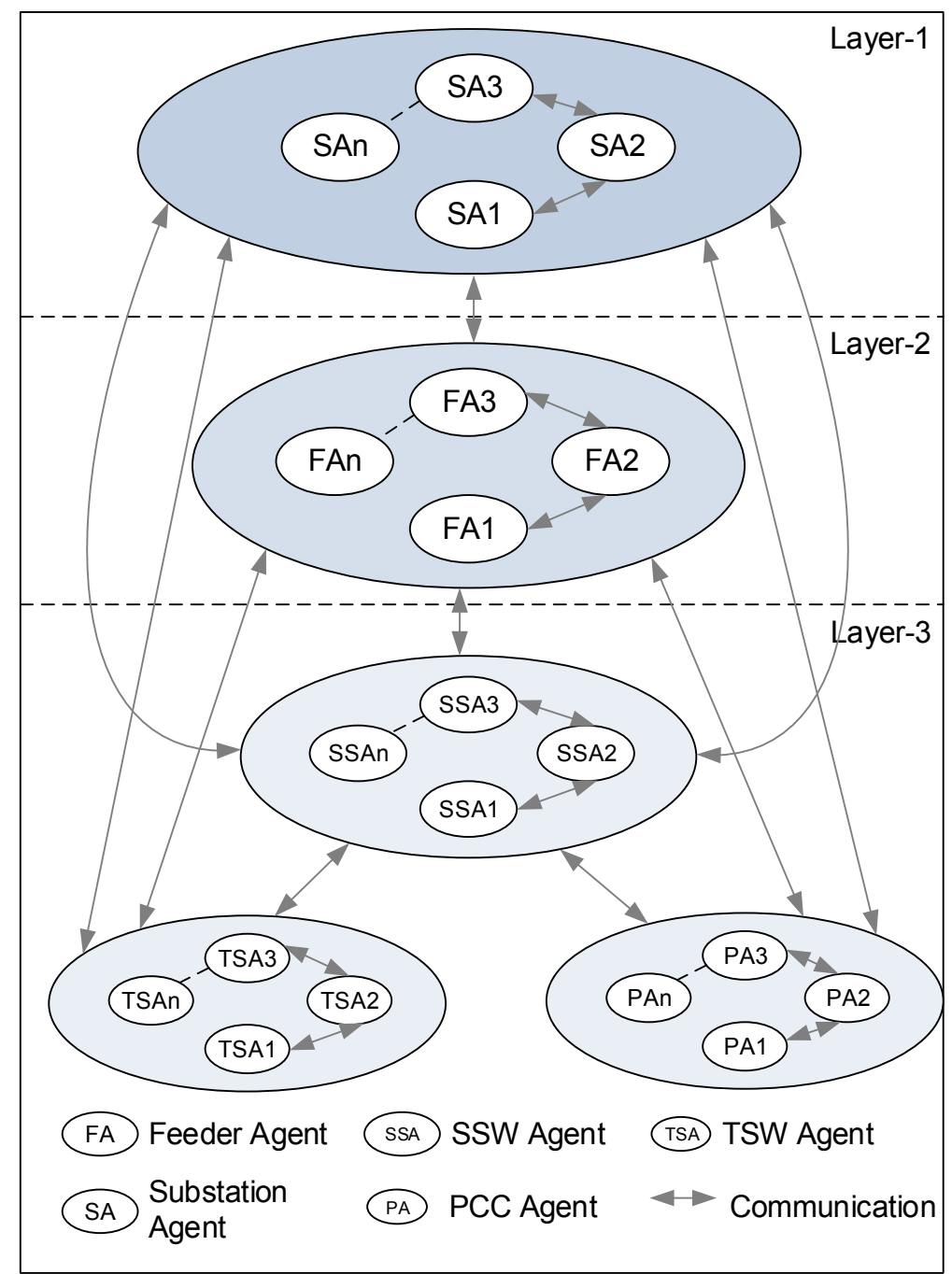

Figure 4-1: Proposed Hybrid MAS Architecture

\subsubsection{Agents}

The agents are intelligent units that have problem solving capabilities and can communicate, resolve, coordinate with other agents to achieve specific FLIR goals. The agents are accommodated in ruggedized computers with required computing power and memory which are powered by Uninterrupted Power Supply (UPS). A typical agent platform has the operating system 
and needed software for communications and protocol conversions. Agent platforms support the following associated functions:

- Execution of advanced FLIR algorithms housed in the agents

- Collection of data in the distribution field and at substations to support modeling, agent learning, and system operations

- Interpretation of data encoded using various protocols including DNP 3.0, Modbus, and IEC-61850

- Translation of data from one message protocol to another to support integration of components utilizing multiple formats and protocols

- Storage of data in the field supporting archival and efficient utilization of communications throughout

- Remote asset monitoring

- Operating system supporting execution of functional source code including C, JAVA, C++, and .NET framework applications

Different types of agents involved in the proposed architecture are explained in detailed below. The actual work that an agent does is carried out in behaviors with different communication acts mentioned as performatives following the ACL format. Table 4-1 provides an exhaustive set of individual agent knowledge, capabilities, and behaviors.

\subsubsection{Substation Agent (SA)}

The SAs at the top layer act as back-up agents for decision making if any of the FA fails to perform its responsibilities. Also, these agents perform supervisory monitoring of the FAs. The SAs mimic FAs in terms of functionalities and capabilities.

\subsubsection{Feeder Agent (FA)}

The FAs and SAs are the main decision making agents, as these agents will have overall system view to optimize the FLIR processes. FA is responsible for monitoring the feeder breaker or 
recloser status and the measured data (current, voltage, real and reactive powers) at its location. FA houses agent learning module which learns from past FLIR actions in a continuous fashion to help with future service restoration events. FA also houses the FLI scheme to locate and isolate the fault that can occur in its own zone. As soon as FA receives Fault Isolation message from one of its SSAs, it starts service restoration of un-faulted zones either by utilizing the agent learning or by mathematical optimization that involves TRA and ZPA algorithms. It can communicate with all the downstream SSAs, TSAs, and PAs in a peer to peer manner to execute the service restoration activities. A detailed functionality diagram is shown in Figure 4-2.

\subsubsection{Sectionalizing Switch Agent (SSA)}

SSA is responsible for monitoring the SSW status and the measured data (current, voltage, real and reactive powers) at its location. SSA houses FLI scheme to locate and isolate the fault that can occur in its own zone. It can communicate with the neighboring SSAs, and TSAs, and PAs present in its zone to compute net pre-fault loading and zone current information. A detailed functionality diagram is shown in Figure 4-2.

\subsubsection{Tie Switch Agent (TSA)}

TSA is responsible for monitoring the TSW status and the measured data (current, voltage, real and reactive powers) at its location. TSA keeps the available feed capacity information. It can communicate with its parent SSA and FA to help with service restoration activities. A detailed functionality diagram is shown in Figure 4-2.

\subsubsection{PCC Agent (PA)}

PA is responsible for monitoring the PCC Switch status and the measured data (current, voltage, real and reactive powers) at its location. It can communicate with its parent SSA and FA to help with FLI and service restoration activities. A detailed functionality diagram is shown in Figure 4-2. 


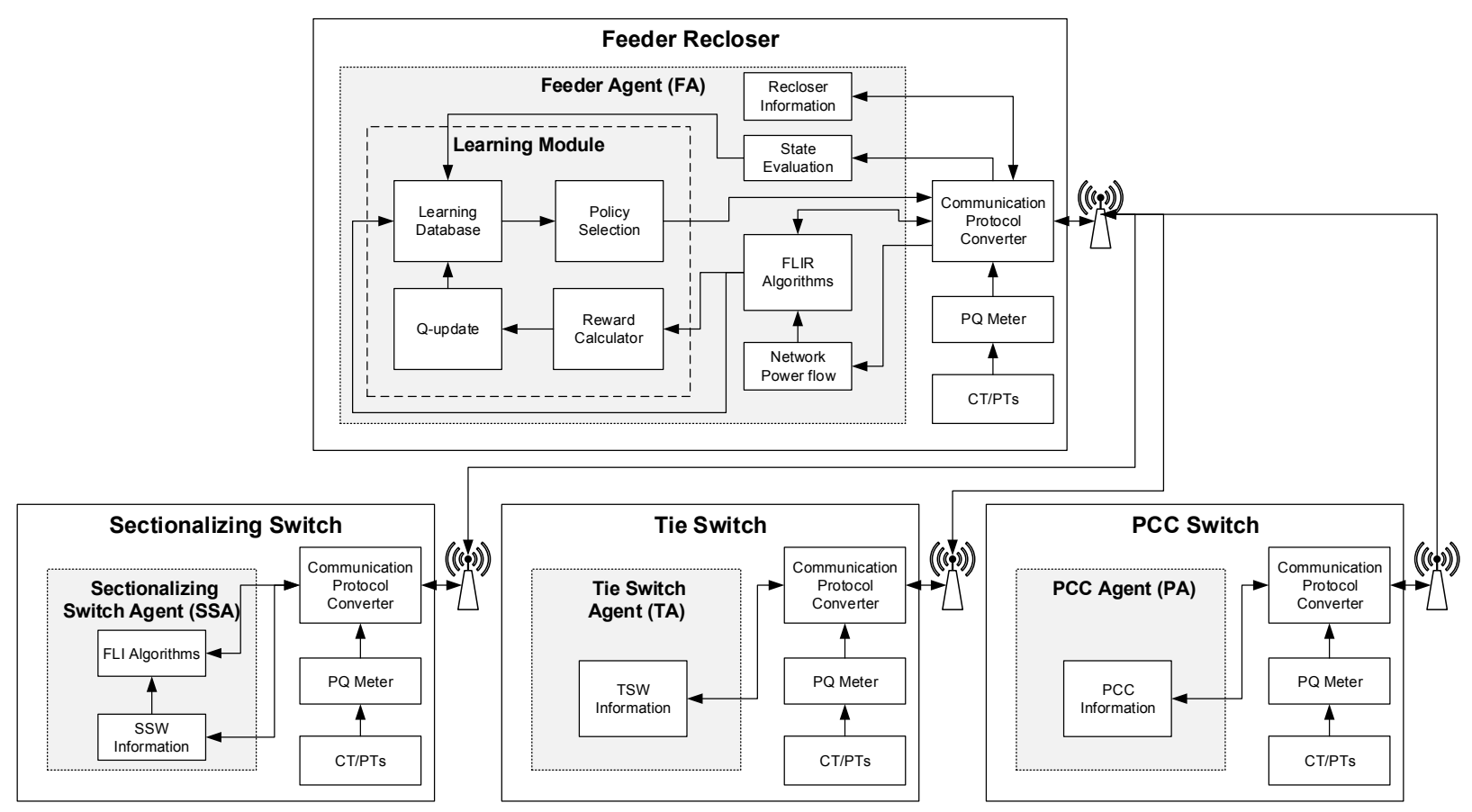

Figure 4-2: Agent Functionality Diagram

Table 4-1: Agent Knowledge/Capabilities and Behaviors

\begin{tabular}{|c|c|c|}
\hline Agent & Knowledge/Capabilities & Behaviors \\
\hline SA & $\begin{array}{l}\text { 1. Knowledge of its children FAs and their } \\
\text { children SSAs, TSAs, and PAs } \\
\text { 2. Knowledge of neighboring SAs } \\
\text { 3. Substation transformer nameplate capacity } \\
\text { (kVA) } \\
\text { 4. System network model } \\
\text { 5. Maintains NEZ_A and NEZ_B lists of its } \\
\text { children FA } \\
\text { 6. Computing power to run power flows, FLI, } \\
\text { service restoration, and agent learning } \\
\text { processes } \\
\text { 7. Agent learning database }\end{array}$ & $\begin{array}{l}\text { Behaviors in normal mode } \\
\text { 1. } Q U E R Y \_R E F \text { behavior to request its children FA } \\
\text { information. } \\
\text { 2. } Q U E R Y \_R E F \text { behavior to check its children FA if it is } \\
\text { alive and performing FLIR tasks. } \\
\text { Behaviors in contingency mode when FA fails } \\
\text { 1. } Q U E R Y R E F \text { behavior to request net pre-fault } \\
\text { loading from its children FA's children SSA. } \\
\text { 2. QUERY } R E F \text { behavior to request its children FA's } \\
\text { children TSA information. } \\
\text { 3. } R E Q U E S T \text { behavior to command its children FA's } \\
\text { children SSA, TSA, or PA to open/close the respective } \\
\text { switch. }\end{array}$ \\
\hline FA & $\begin{array}{l}\text { 1. Knowledge of its parent SA } \\
\text { 2. Knowledge of its neighboring FAs } \\
\text { 3. Knowledge of its children SSAs, TSAs, } \\
\text { and PAs } \\
\text { 4. Knowledge of local measurements voltage } \\
\text { (V), current (A), real power (kW), and } \\
\text { reactive power (kvar) } \\
\text { 5. Knowledge of feeder recloser or breaker } \\
\text { status (open/close) }\end{array}$ & $\begin{array}{l}\text { 1. INFORM behavior to send information to its parent } \\
\text { SA. } \\
\text { 2. } Q U E R Y \_R E F \text { behavior to request its neighboring FA } \\
\text { information. } \\
\text { 3. INFORM behavior to send requested information to } \\
\text { its neighboring FA. } \\
\text { 4. } Q U E R Y \_R E F \text { behavior to request net pre-fault } \\
\text { loading from its children SSA. }\end{array}$ \\
\hline
\end{tabular}




\begin{tabular}{|c|c|c|}
\hline & $\begin{array}{l}\text { 6. Maintains NEZ_A and NEZ_B lists } \\
\text { 7. System network model } \\
\text { 8. Computing power to run power flows, FLI, } \\
\text { service restoration, and agent learning } \\
\text { processes } \\
\text { 9. Agent learning database }\end{array}$ & $\begin{array}{l}\text { 5. QUERY_REF behavior to request its children SSAs to } \\
\text { initiate FLI process. } \\
\text { 6. QUERY_REF behavior to request its children TSA } \\
\text { information. } \\
\text { 7. REQUEST behavior to command its children SSA, } \\
\text { TSA, or PA to open/close the respective switch. }\end{array}$ \\
\hline SSA & $\begin{array}{l}\text { 1. Knowledge of its parent FA and SA } \\
\text { 2. Knowledge of its neighboring SSAs } \\
\text { 3. Knowledge of TSAs, and PAs in its zone } \\
\text { 4. Knowledge of local measurements voltage } \\
\text { (V), current (A), real power (kW), and } \\
\text { reactive power (kvar) } \\
\text { 5. Knowledge of SSW status (open/close) } \\
\text { 6. Computing power to run FLI processes }\end{array}$ & $\begin{array}{l}\text { 1. INFORM behavior to send net pre-fault loading } \\
\text { information to its parent FA or SA. } \\
\text { 2. INFORM behavior to send FLI information to its } \\
\text { parent FA or SA. } \\
\text { 3. ACCEPT behavior to perform requested switching } \\
\text { action by its parent FA or SA. } \\
\text { 4. QUERY_REF behavior to request its pre-fault } \\
\text { measurements from TSAs and PAs in its zone. } \\
\text { 5. } Q U E R Y \text {. } R E F \text { behavior to request pre-fault } \\
\text { measurements from its neighboring SSAs. } \\
\text { 6. INFORM behavior to send requested information to } \\
\text { its neighboring SSA. } \\
\text { 7. REQUEST behavior to command its neighboring SSA } \\
\text { to open/close the respective switch. } \\
\text { 8. ACCEPT behavior to perform requested switching } \\
\text { action by its neighboring SSA. }\end{array}$ \\
\hline TSA & $\begin{array}{l}\text { 1. Knowledge of its parent FA, SA, and SSA } \\
\text { 2. Knowledge of its neighboring TSAs and } \\
\text { PAs } \\
\text { 3. Knowledge of local measurements voltage } \\
\text { (V), current (A), real power (kW), and } \\
\text { reactive power (kvar) } \\
\text { 4. Knowledge of TSW type and Available } \\
\text { Feed Capacity (AFC) } \\
\text { 5. Knowledge of TSW status (open/close) }\end{array}$ & $\begin{array}{l}\text { 1. INFORM behavior to send pre-fault measurements } \\
\text { information to its parent SSA. } \\
\text { 2. ACCEPT behavior to perform requested switching } \\
\text { action by its parent FA or SA. }\end{array}$ \\
\hline PA & $\begin{array}{l}\text { 1. Knowledge of its parent FA, SA, and SSA } \\
\text { 2. Knowledge of its neighboring TSAs and } \\
\text { PAs } \\
\text { 3. Knowledge of local measurements voltage } \\
\text { (V), current (A), real power (kW), and } \\
\text { reactive power (kvar) } \\
\text { 4. Knowledge of DG type (synchronous } \\
\text { generator, induction generator, or inverter } \\
\text { based DG) } \\
\text { 5. Knowledge of facility type (individual DG, } \\
\text { or microgrid) } \\
6 . \text { Knowledge of PCC switch status } \\
\text { (open/close) }\end{array}$ & $\begin{array}{l}\text { 1. INFORM behavior to send pre-fault measurements } \\
\text { information to its parent SSA. } \\
\text { 2. ACCEPT behavior to perform requested switching } \\
\text { action by its parent FA or SA. }\end{array}$ \\
\hline
\end{tabular}




\subsection{WVSC MAS Design}

Figure 4-3 shows the WVSC distribution system, which is used to demonstrate the proposed MAS FLIR solution with all agent placements. The targeted system for DA involves two substations, WestRun and Pierpont substations. The WestRun substation has six outgoing feeders and each feeder is protected by a recloser. The FAs are placed right at these recloser locations. The WR\#3 and WR\#4 feeders are strategically divided into several zones by SSWs and these zones are connected to adjacent feeders through a set of TSWs. The SSAs and TSAs are placed at these switch locations. In order to demonstrate the DG and microgrid elements of the proposed FLIR solution a few fictitious DG and microgrid points are introduced in WR\#3 feeder. The PAs are placed right at the PCC switches of these DG and microgrid resources.

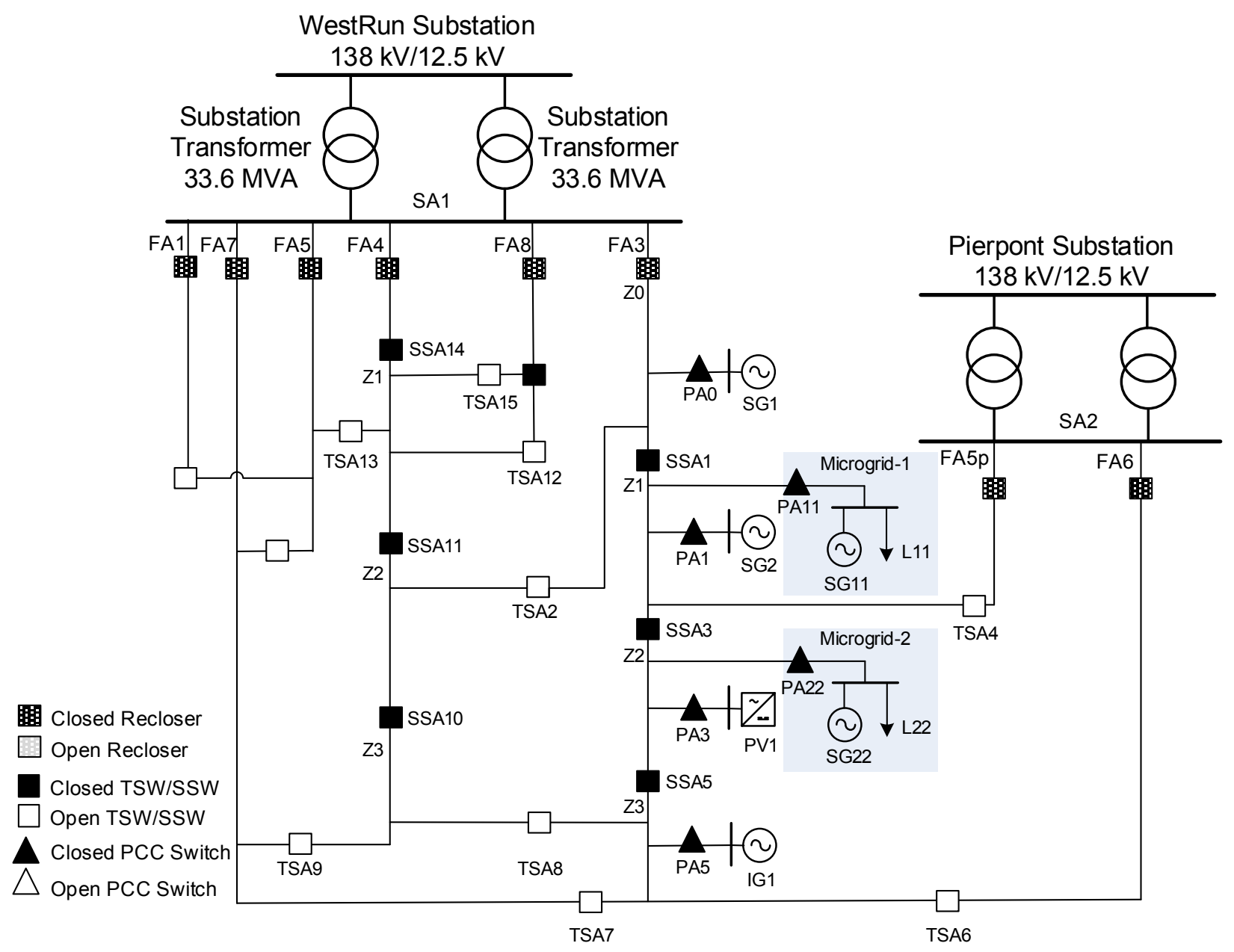

Figure 4-3: Agent Placement for WVSC Distribution System 


\subsection{Agent State Transition}

Agents in the proposed MAS framework transition from one state to another as shown in the state transition diagram in Figure 4-4. Agent can be in one of the following states at any given point of time. The supervisory agents SA and FA make sure that all the agents in the MAS update their state according to the system conditions. It is quite essential for agents to maintain these states as it helps proper coordination among the agents to achieve the organizational goals of FLIR as a single team.

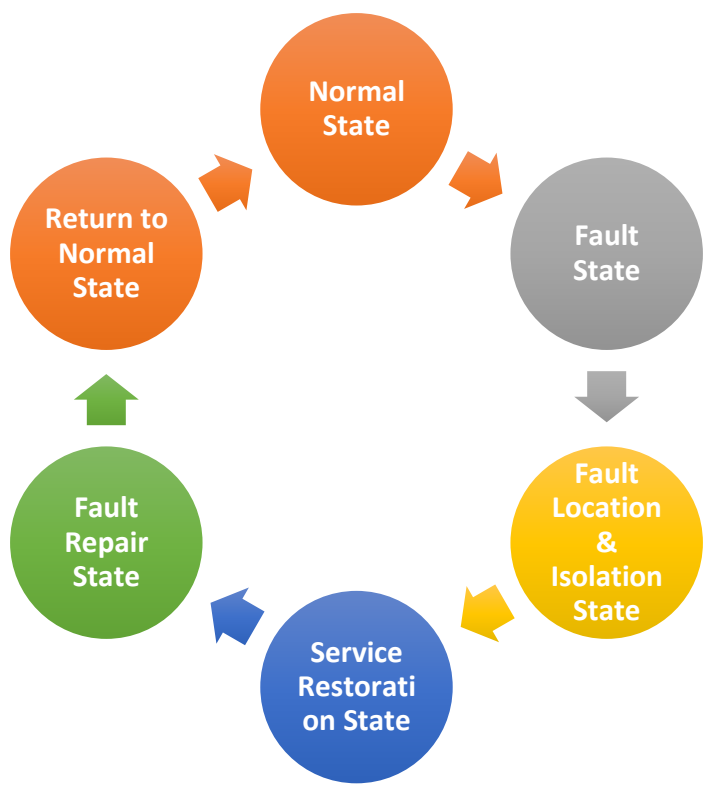

Figure 4-4: Agent State Transition

- Normal State: Agents stay in this state when the system they are connected to is operating normally without any faults or contingencies. Agents spend most of their time in this state and continuously monitor/record the data, and update the learning database if they have the learning ability.

- Fault State: Agents transition to this state when there is a permanent fault in the electric distribution system.

- Fault Location and Isolation State: Agents transition to this state when the fault location and isolation process takes place right after the feeder recloser/CB locks-out. 
- Service Restoration State: Agents transition to this state when the service restoration process takes place soon after fault has been isolated.

- Fault Repair State: Agents transition to this state when the fault is being repaired.

- Return to Normal State: Agents transition to this state when the fault is fixed and distribution operator wants to bring back the system to the normal state.

\subsection{Proposed FLIR Process Flow}

Figure 4-5 shows the process flow diagram of the proposed FLIR solution. As soon as a permanent fault occurs on the electric distribution system the whole system transitions into the fault state, and the feeder protection system operates to clear the fault. The permanent lock-out of the feeder recloser/CB initiates the MAS to transition into the FLI state where all the zones on the faulty feeder would isolate in preparation for service restoration. The SSAs of the faulty feeder employ the "Fault Detection Algorithm" and with the help of coordinated agent communications the faulted zone is identified and isolated. This triggers service restoration state, where the fault-free zones that have no power are restored either from a potential solution from the "Agent Learning Database" or by performing a restoration optimization.

The agents involved in the FLIR MAS are capable of perceiving the system conditions and learn in a continuous fashion. Each time a successful FLIR action is taken by MAS the "Agent Learning Database" would be updated with the MAS action and pre and post system state. If there is no feasible solution exist in the "Agent Learning Database", the concerned FA or SA performs restoration optimization using "Tie-Switch Ranking Algorithm" and "Zone Priority Algorithm" to find out the best possible restoration plan. The main objective of the restoration optimization is to restore as many fault-free zones as possible using all the available Tie-switch options. The optimization also accounts for existing DG units and microgrids. Once the solution is discovered, the FA/SA initiates commands to its downstream agents to implement the restoration plan. The next stage is where the utility personnel repair the faulty portion or equipment of the system. Once the fault is cleared, the distribution operator initiates MAS return to normal procedure. The MAS follows the proposed procedure to bring back the system to normal state. 


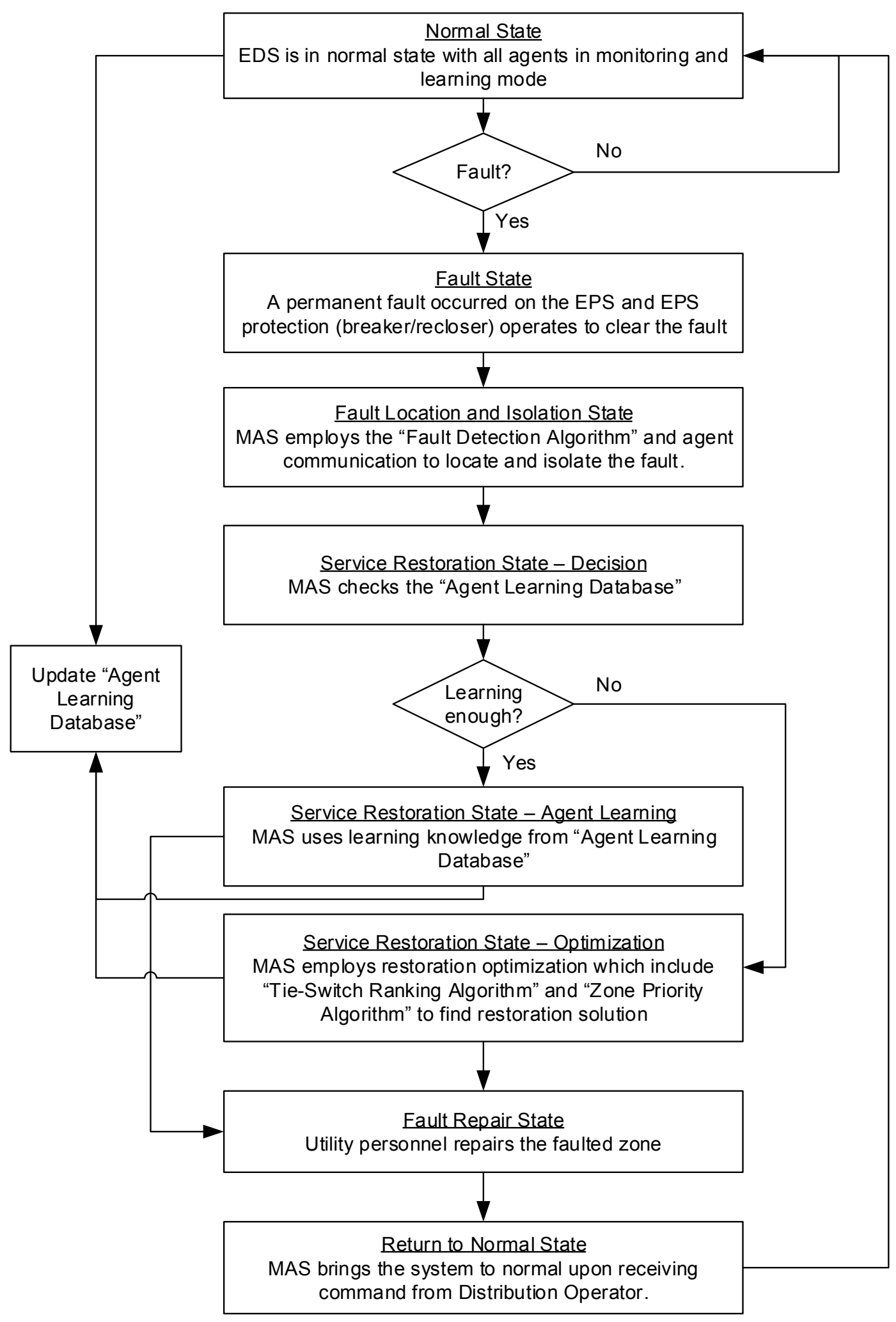

Figure 4-5: Proposed FLIR Process Flow 


\section{Chapter 5}

\section{Switch Optimization}

A novel iterative approach is proposed based on relative reduction in the normalized customer interruption costs for the optimal switch number and placement problem. An iterative algorithm is constructed which minimizes the total interruption costs at each step of the analysis to arrive at the solution. The proposed approach isolates the impacts of varying switch investment and customer interruption costs that are usually based on outdated utility surveys.

The proposed approach is generalized and works for all kinds of switch types, such as load break switches, reclosers, fault breaking circuit breakers, etc., with various levels of automation, such as manually operated, motor operated, remotely operated, etc. Switch investments are isolated in the approach as these are dependent on switch type and manufacturer, and also vary from country to country. The proposed iterative algorithm does not rely on absolute customer interruption costs which are largely dependent on customer damage functions. Customer damage functions usually are derived based on various survey methods, and they vary greatly from one method of estimation to the other. This makes determination of optimal switch number and placement very subjective when the absolute values of customer interruption costs are used in the analysis. Also, this may lead to unrealistic number of switches as the optimal solution. The proposed iterative algorithm isolates the impacts of such customer damage functions by employing an approach based on relative reduction in the normalized customer interruption costs. 


\subsection{Mathematical Problem Formulation}

The proposed iterative algorithm for the optimum switch number and placement problem makes use of customer interruption cost (CIC) [67]. CIC responds to the effects of system topology, interruption duration, load variations, and component random failures. It also recognizes various customer types and their nonlinear customer damage functions.

DA distribution feeder is divided into a set of Super Sections (SS). SS are formed by logically grouping a set of actual feeder line segments. Each SS will have load points and the equivalent load of SS is obtained by summing up individual loads in the SS. SS must be strategically selected in a way that the created zones of the feeder will have the ability to restore the power from adjacent feeders in the event of outages. The restoration of the power must not violate the system constraints such as thermal constraints (conductor or equipment loading limits) and voltage constraints (low and high voltage issues). DA feeders can be divided into SS with un-equal circuit lengths and loadings. Each SS is considered as a potential automated switch location in the analysis. A typical distribution feeder that is divided into SS is shown in Figure 5-1. CIC used in this study is expressed as:

$$
\begin{gathered}
C I C=\sum_{\mathrm{y}=1}^{\mathrm{k}} \mathrm{OC}_{\mathrm{y}}=\sum_{\mathrm{y}=1}^{\mathrm{k}} \xi_{\mathrm{y}} \mathrm{l}_{\mathrm{y}}\left(\sum_{\mathrm{z}=1}^{\mathrm{k}} \mathrm{C}_{\mathrm{yz}} \mathrm{P}_{\mathrm{z}}\right) \\
C_{y z}=\left(\operatorname{Res}_{z} * f_{R}\left(r_{y z}\right)+\operatorname{Com}_{z} * f_{C}\left(r_{y z}\right)+\operatorname{Ind}_{z} * f_{I}\left(r_{y z}\right)\right)
\end{gathered}
$$

Where,

$k \quad$ Total number of Super Sections

$O C_{y} \quad$ Interruption cost per year due to outages in $S S-y$

$\xi_{y} \quad$ Outage rate (failure/mile-year) of $S S-y$

$l_{y} \quad$ Circuit length in miles of SS-y

$C_{y z} \quad$ Interruption cost of load (residential, commercial and industrial customers) in $\$ / k W$ at $S S-z$ due to an outage at $S S-y$

$P_{z} \quad$ Total load in $k W$ of $S S-z$

$\operatorname{ReS}_{Z} \quad$ Load percentage of residential customers at SS-z

$\mathrm{Com}_{z} \quad$ Load percentage of commercial customers at $S S-z$

Ind $_{z} \quad$ Load percentage of industrial customers at SS-z

$f_{R} \quad$ Interruption cost function of residential customers 
$f_{C} \quad$ Interruption cost function of commercial customers

$f_{I} \quad$ Interruption cost function of industrial customers

$r_{y z} \quad$ Duration of service interruption of SS-z due to an outage at SS-y

The integrated interruption cost framework given in equation (5.2) can be expanded to other types of customers as long as the customer damage functions are known. Customer interruption costs are heavily reliant on customer type and outage duration. For example, the interruption cost of residential customers is far less compared to industrial customers. The customers with high service priorities such as hospitals, police stations, fire stations, and tele-communication data centers have high interruption costs.

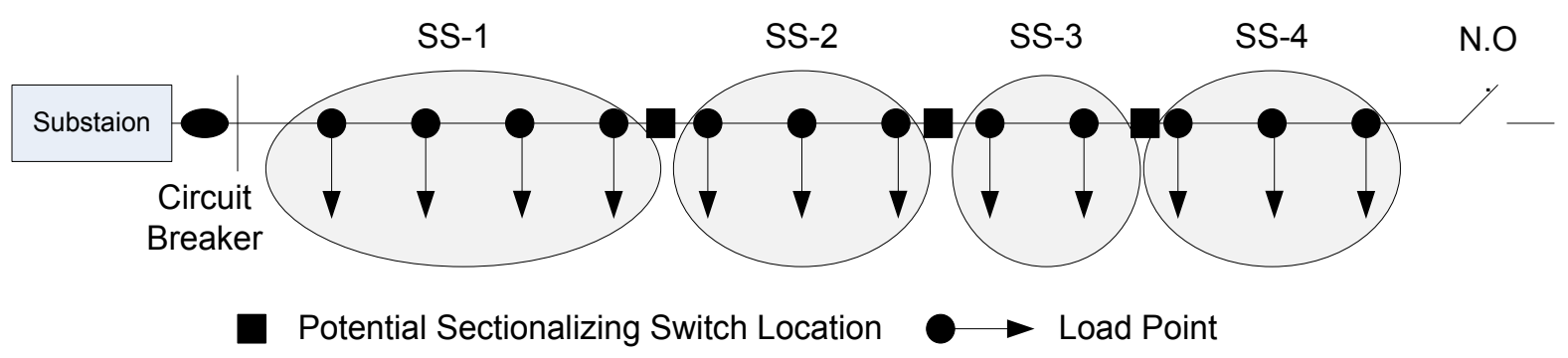

Figure 5-1: Typical Radial Distribution Feeder

\subsection{Iterative Algorithm for Optimal Switch Number and Placement}

The customer interruption cost $(C I C)$ is calculated for all possible combinations of switch placements with different number of switches placed in the system. A generalized formula for total number of possible combinations the CIC is calculated for a feeder with "n" number of SS for placing "r" number of automated switches is given by,

$$
\sum_{r=1}^{(n-1)} C_{r}^{(n-1)}=\sum_{r=1}^{(n-1)} \frac{(n-1) !}{r !(n-1-r) !}
$$


The proposed approach significantly reduces the search space and the possible switch combinations for which $C I C$ needs to be computed. The determination of optimal switch number and location is achieved by the iterative algorithm shown in Figure 5-2.

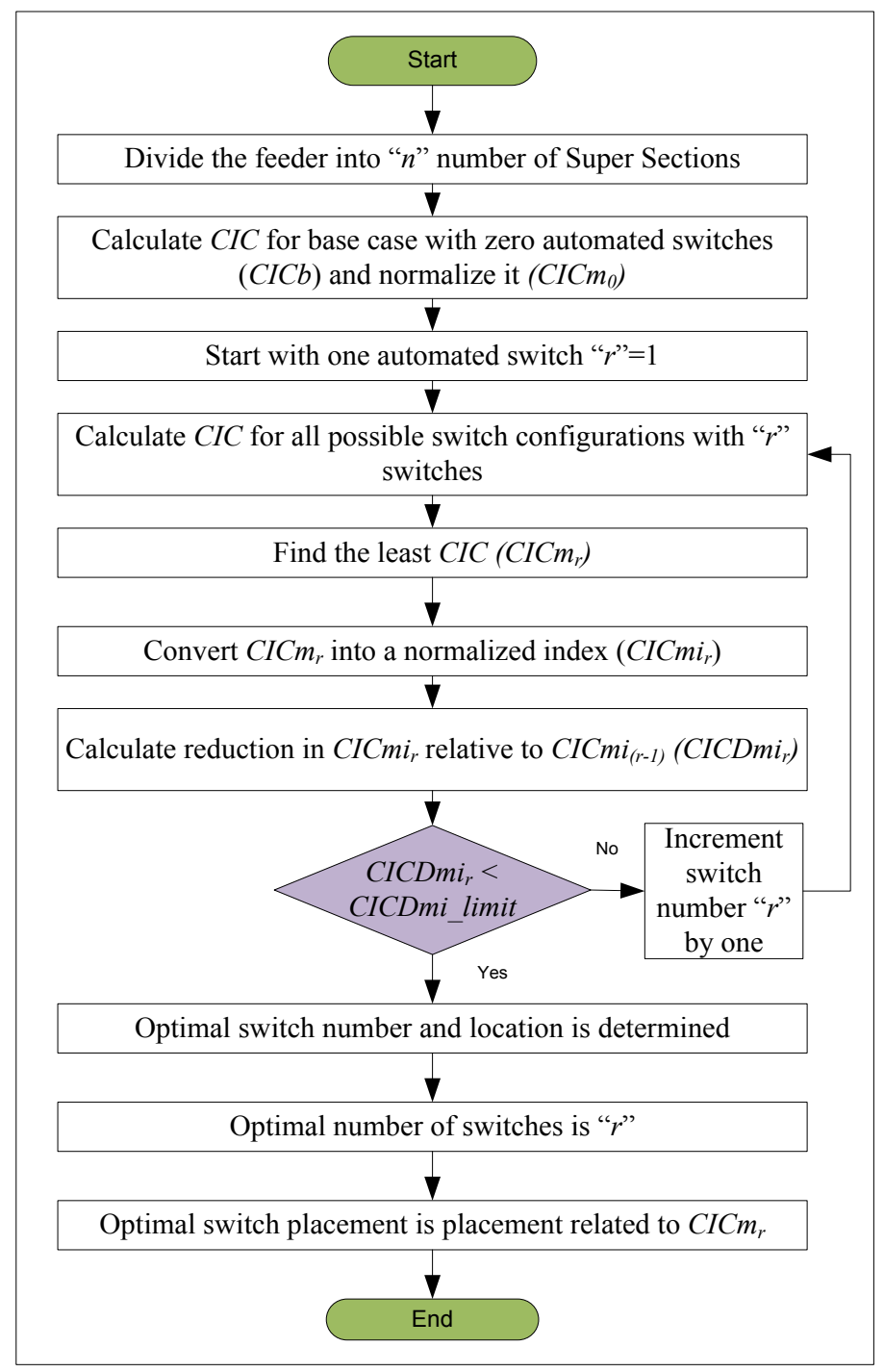

Figure 5-2: Iterative Algorithm for Optimal Switch Number and Placement

The steps involved in the process are explained below,

Step 1: Divide the DA feeder into " $n$ " number of SS.

Step 2: Calculate $C I C$ for the base case feeder with zero automated switches $(C I C b)$.

Normalize this interruption cost to find cost index $\left(\mathrm{CICmi}_{0}\right)$. 
Step 3: Start the iterative process with one automated switch " $r$ " $=1$.

Step 4: Calculate CIC for all possible switch configurations with " $r$ " switches. The number of possible combinations is given by equation (5.3).

Step 5: Find the least $C I C$ among all the possible switch configurations $\left(C I C m_{r}\right)$ with " $r$ " switches.

Step 6: Normalize $\mathrm{CICm}_{r}$ to find cost index $\left(\mathrm{CICmi}_{r}\right)$.

Step 7: Calculate reduction in $\mathrm{CICmi}_{r}$ relative to $\mathrm{CICmi} i_{(r-1)}\left(\mathrm{CICDmi}_{r}\right)$

Step 8: Check if $C I C D m i_{r}$ is less than the iterative threshold $\left(C I C D m i_{\text {limit }}\right)$. Although this is a configurable parameter, a value of " 8 " is used as the threshold in this study.

Step 9: If the condition is FALSE, then increment the number of switches " $r$ " by one and repeat the process from Step 4.

Step 10: If the condition is TRUE, then the iterative process has identified the optimal number and placement of switches. The optimal number of switches is " $r$ ". The optimal placement is the placement corresponding to $\mathrm{CICm}_{r}$.

\subsection{Test System and Results}

The proposed iterative algorithm for optimal switch number and placement is tested on IEEE 34bus and IEEE 123-bus test feeders, and Mon Power's real distribution network involved in WVSC project.

\subsubsection{Test System (IEEE 34-Bus System)}

The IEEE 34-bus test feeder is an actual feeder located in Arizona, with a nominal voltage of 24.9 $\mathrm{kV}$. This is a lengthy feeder and assumed to have poor reliability which makes it a perfect candidate to implement the proposed solution. The data pertaining to feeder load (spot loads and distributed loads) and line segments (overhead and underground lines) are taken from [68]. The feeder has a total of 33 line segments with 6 spot loads and 19 distributed loads.

This feeder is strategically divided into seven SS as shown in Figure 5-3. Circuit topography, customer distribution through the feeder, tie-points and switch locations were taken into account in the SS selection process. Each SS location is considered to be a potential location for sectionalizing switch. The information relevant to each SS is furnished in Table 5-1. The information includes circuit length, load, and failure rate per annum per mile. The circuit length is different from the actual feeder length in the sense that circuit length is the actual conductor length 
of line segment. For example, a three phase line segment's circuit length would be three times the actual line segment's length. This helps to account the fault rate to each phase of the line segments within each SS. As the reliability data for this feeder is not available typical values shown in Table 5-1 are used in the analysis. Each SS is assumed to have a customer mix composed of 50\% residential customers, $25 \%$ commercial, and 25\% industrial customers.

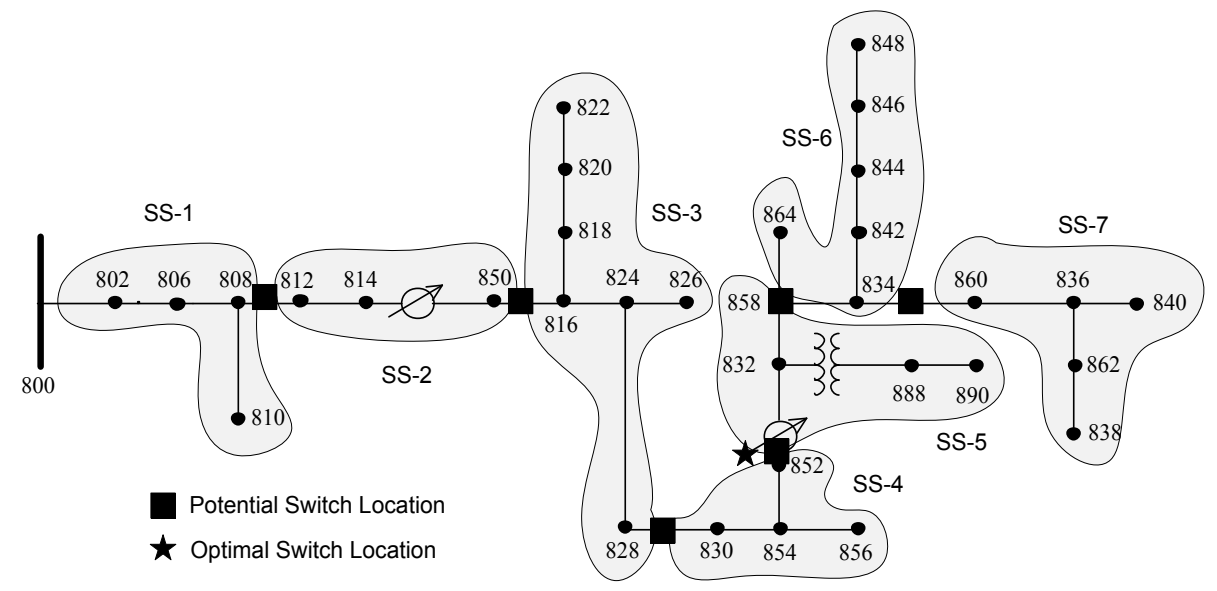

Figure 5-3: IEEE 34-Bus Test Feeder

Table 5-1: IEEE 34-Bus and 123-Bus Test Feeder SS Data

\begin{tabular}{|c|c|c|c|c|c|c|}
\hline & \multicolumn{2}{|c|}{ IEEE 34-bus system } & \multicolumn{2}{c|}{ IEEE 123-bus system } \\
\hline $\begin{array}{c}\text { Super } \\
\text { Section } \\
\text { ID }\end{array}$ & $\begin{array}{c}\text { Circuit } \\
\text { Length } \\
\text { (miles) }\end{array}$ & $\begin{array}{c}\text { Load } \\
\text { (kVA) }\end{array}$ & $\begin{array}{c}\text { Failure } \\
\text { Rate / } \\
\text { year- } \\
\text { mile }\end{array}$ & $\begin{array}{c}\text { Circuit } \\
\text { Length } \\
\text { (miles) }\end{array}$ & $\begin{array}{c}\text { Load } \\
\text { (kVA) }\end{array}$ & $\begin{array}{c}\text { Failure } \\
\text { Rate / } \\
\text { year- } \\
\text { mile }\end{array}$ \\
\hline SS-1 & 21.86 & 80.08 & 0.06 & 1.17 & 335.41 & 0.59 \\
\hline SS-2 & 38.20 & 0.00 & 0.06 & 4.67 & 1406.16 & 0.59 \\
\hline SS-3 & 19.07 & 244.66 & 0.06 & 1.02 & 178.89 & 0.59 \\
\hline SS-4 & 37.25 & 61.37 & 0.06 & 1.23 & 449.38 & 0.59 \\
\hline SS-5 & 9.10 & 521.91 & 0.06 & 3.47 & 626.10 & 0.59 \\
\hline SS-6 & 6.61 & 712.50 & 0.06 & 0.31 & 438.21 & 0.59 \\
\hline SS-7 & 4.24 & 442.92 & 0.06 & 1.85 & 290.69 & 0.59 \\
\hline SS-8 & N/A & N/A & N/A & 1.06 & 268.33 & 0.59 \\
\hline
\end{tabular}




\subsubsection{Test System (IEEE 123-Bus System)}

The IEEE 123-bus feeder is considered as a test system to be able to show the computational efficacy of the proposed solution on a more complicated network. The feeder operates at a nominal voltage of $4.16 \mathrm{kV}$ and is characterized by overhead and underground lines, unbalanced loading, four voltage regulators, and shunt capacitor banks. The data pertaining to feeder load and line segments are taken from [68]. This feeder is strategically divided into eight SS as shown in Figure 5-4. The information relevant to each SS is furnished in Table 5-1. Similar assumptions and data used for the prior test case are used for this test system.

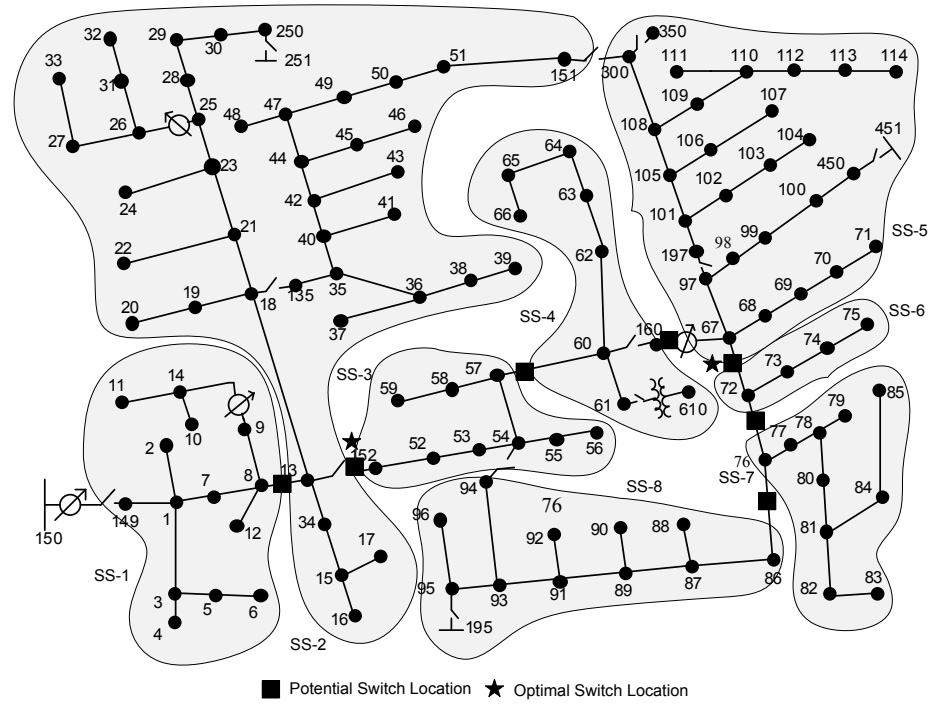

Figure 5-4: IEEE 123-Bus Test Feeder

\subsubsection{Test System (WVSC Distribution System)}

Two distribution circuits in Morgantown, WV that were part of WVSC project are selected as the test system, mainly due to their poor reliability. The geographic view of the Morgantown circuits is illustrated in Figure 5-5. The two circuits are the West Run \#3 Stewarts Street and the West Run \#4 Pine View Circuits. The West Run \#3 circuit has 5 normally open tie points with adjacent circuits. Likewise, West Run \#4 has also 5 normally open tie points with adjacent circuits. 
Therefore, both circuits have many alternative sources which can be utilized to provide service after a fault condition has been successfully isolated.

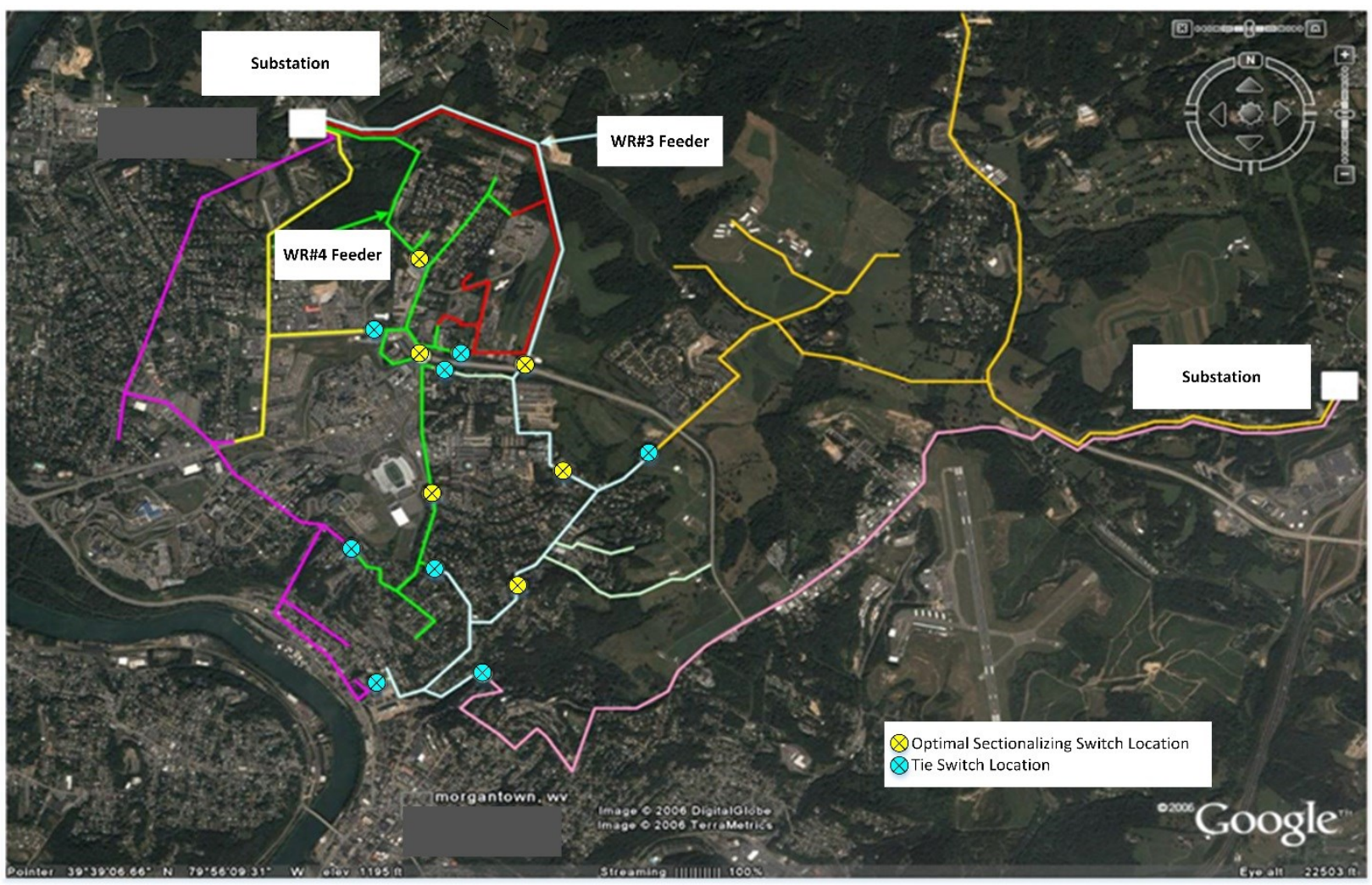

Figure 5-5: Geographic View of WVSC Feeders

The West Run substation has two $138 / 12.5 \mathrm{kV}$ transformers, each with an installed capacity of 33.6 MVA. The Pierpont substation has two $138 / 12.5 \mathrm{kV}$ transformers with each having a capacity of 22.4 MVA. There are 327 distribution transformers installed on the West Run \#3 circuit and 341 distribution transformers installed on the West Run \#4 circuit. The sum of the capacities of the distribution transformers installed on the West Run \#3 circuit is $12,942 \mathrm{kVA}$, while the sum of the capacities of the distribution transformers installed on the West Run \#4 circuit is 16,670 kVA. The number of transformers, according to their installed capacities, is illustrated in Figure 5-6. 


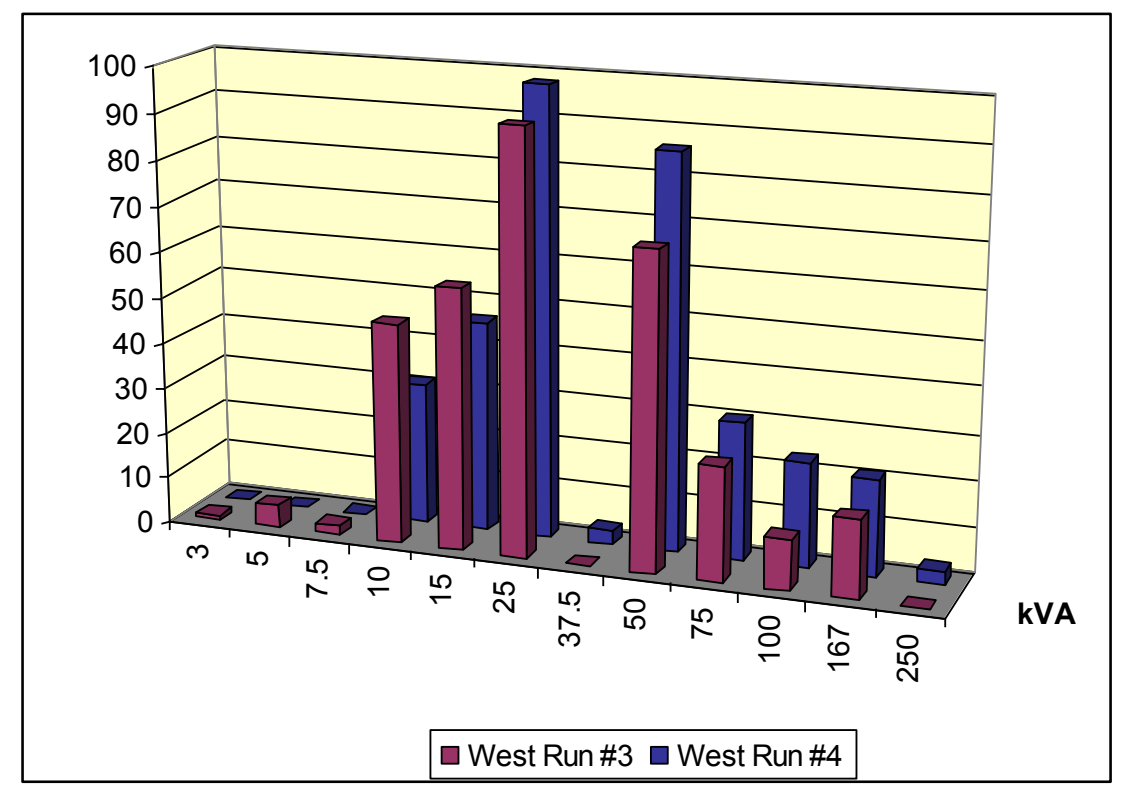

Figure 5-6. WR\#3 and WR\#4 Distribution Transformer Data

The West Run \#3 circuit has 5.85 miles of 3-phase, 1.28 miles of open-wye circuits and 7.39 miles of single phase circuits. The West Run \#4 circuit has 6.02 miles of 3-phase, 0.22 miles of openwye circuits and 10.08 miles of single phase circuits. Both the circuits are dominated by residential type loads. The West Run \#3 circuit has 2,452 customers (2,336 residential, 111 commercial and 5 industrial). Similarly, the West Run \#4 circuit has 3,032 customers (2,804 residential, 219 commercial and 9 industrial).

The circuits are protected by Cooper Electronic Reclosers with Form- 6 controllers at the West Run substation. There are no other reclosers on the main line or on the tap lines on either circuit. Table 5-2 presents historic reliability data in the form of reliability indices SAIDI, CAIDI, and SAIFI for WR\#3 and WR\#4 circuits.

Table 5-2. Reliability Indices of WVSC Circuits (Major Events Included)

\begin{tabular}{|l|l|l|l|l|l|l|}
\hline CAIDI & 2003 & 2004 & 2005 & 2006 & 2007 & 2008 \\
\hline West Run \#3 & 149.00 & 655.48 & 1387.33 & 138.15 & 105.63 & 84.88 \\
\hline West Run \#4 & 177.41 & 285.23 & 1847.76 & 85.10 & 132.74 & 78.66 \\
\hline
\end{tabular}




\begin{tabular}{|c|c|c|c|c|c|c|}
\hline West Run \#4 & 53.81 & 102.58 & 929.50 & 11.11 & 17.89 & 277.28 \\
\hline SAIFI & 2003 & 2004 & 2005 & 2006 & 2007 & 2008 \\
\hline West Run \#3 & 1.2892 & 0.3031 & 1.5807 & 0.2620 & 0.2484 & 2.1538 \\
\hline West Run \#4 & 0.3032 & 0.3594 & 0.5079 & 0.1305 & 0.1347 & 3.5252 \\
\hline
\end{tabular}

The WR\#3 and WR\#4 circuits are strategically divided into 16 SS and 15 SS respectively. Each SS location is considered to be a potential location for sectionalizing switch. The information relevant to each SS is furnished in Table 5-3. The load data is only available at the substation transformer in 15-minute intervals. Therefore, it is assumed that transformer load is distributed to the feeders evenly proportional with the feeders' distribution transformer installed capacities. It is assumed that customer load percentages at all SS are equal to each other, and the customer mix at the feeder level are used as the input data. The customer load percentages $\left(\operatorname{ReS}_{z}, \operatorname{Com}_{z}, \operatorname{Ind}_{z}\right)$ for WR\#3 and WR\#4 feeder used in the analysis are $(0.95,0.04,0.01)$ and $(0.92,0.07,0.00)$ respectively.

Table 5-3: WR\#3 and WR\#4 Feeder SS Data

\begin{tabular}{|c|c|c|c|c|c|c|}
\hline & \multicolumn{3}{|c|}{ WR\#3 Feeder } & \multicolumn{3}{c|}{ WR\#4 Feeder } \\
\cline { 2 - 7 } $\begin{array}{c}\text { Super } \\
\text { Section } \\
\text { ID }\end{array}$ & $\begin{array}{c}\text { Circuit } \\
\text { Length } \\
\text { (miles) }\end{array}$ & $\begin{array}{c}\text { Load } \\
\text { (kVA) }\end{array}$ & $\begin{array}{c}\text { Failure } \\
\text { Rate / } \\
\text { year- } \\
\text { mile }\end{array}$ & $\begin{array}{c}\text { Circuit } \\
\text { Length } \\
\text { (miles) }\end{array}$ & $\begin{array}{c}\text { Lailure } \\
\text { (kVA) } \\
\text { Rate / } \\
\text { year- } \\
\text { mile }\end{array}$ \\
\hline SS-1 & 0.95 & 463 & 0.0356 & 0.82 & 651 & 0.0364 \\
\hline SS-2 & 1.20 & 274 & 0.0356 & 1.03 & 385 & 0.0364 \\
\hline SS-3 & 1.19 & 89 & 0.0356 & 1.03 & 125 & 0.0364 \\
\hline SS-4 & 0.98 & 164 & 0.0356 & 0.84 & 230 & 0.0364 \\
\hline SS-5 & 1.35 & 1249 & 0.0356 & 1.16 & 1755 & 0.0364 \\
\hline SS-6 & 2.94 & 1309 & 0.0356 & 5.06 & 3681 & 0.0364 \\
\hline SS-7 & 2.94 & 1309 & 0.0356 & 0.36 & 482 & 0.0364 \\
\hline SS-8 & 0.41 & 343 & 0.0356 & 1.30 & 960 & 0.0364 \\
\hline SS-9 & 1.50 & 683 & 0.0356 & 1.48 & 1307 & 0.0364 \\
\hline SS-10 & 1.72 & 930 & 0.0356 & 1.56 & 1240 & 0.0364 \\
\hline SS-11 & 1.81 & 882 & 0.0356 & 1.76 & 1875 & 0.0364 \\
\hline SS-12 & 2.04 & 1334 & 0.0356 & 1.55 & 2599 & 0.0364 \\
\hline SS-13 & 1.80 & 1849 & 0.0356 & 1.36 & 1132 & 0.0364 \\
\hline SS-14 & 1.58 & 806 & 0.0356 & 1.86 & 610 & 0.0364 \\
\hline
\end{tabular}




\begin{tabular}{|c|c|c|c|c|c|c|}
\hline SS-15 & 2.16 & 434 & 0.0356 & 2.38 & 1155 & 0.0364 \\
\hline SS-16 & 2.76 & 822 & 0.0356 & N/A & N/A & N/A \\
\hline
\end{tabular}

\subsubsection{Data Assumptions}

Average service duration of 240 minutes and automated switch operating duration of 1 minute are used in this study for all the test systems. The underlying assumption in the analysis is that each SS has back feed capability from an adjacent feeder to restore power to the interrupted customers in the event of outage. The customer damage functions for residential, commercial, and industrial customer classes shown in Figure 5-7 [17] are used for this study.

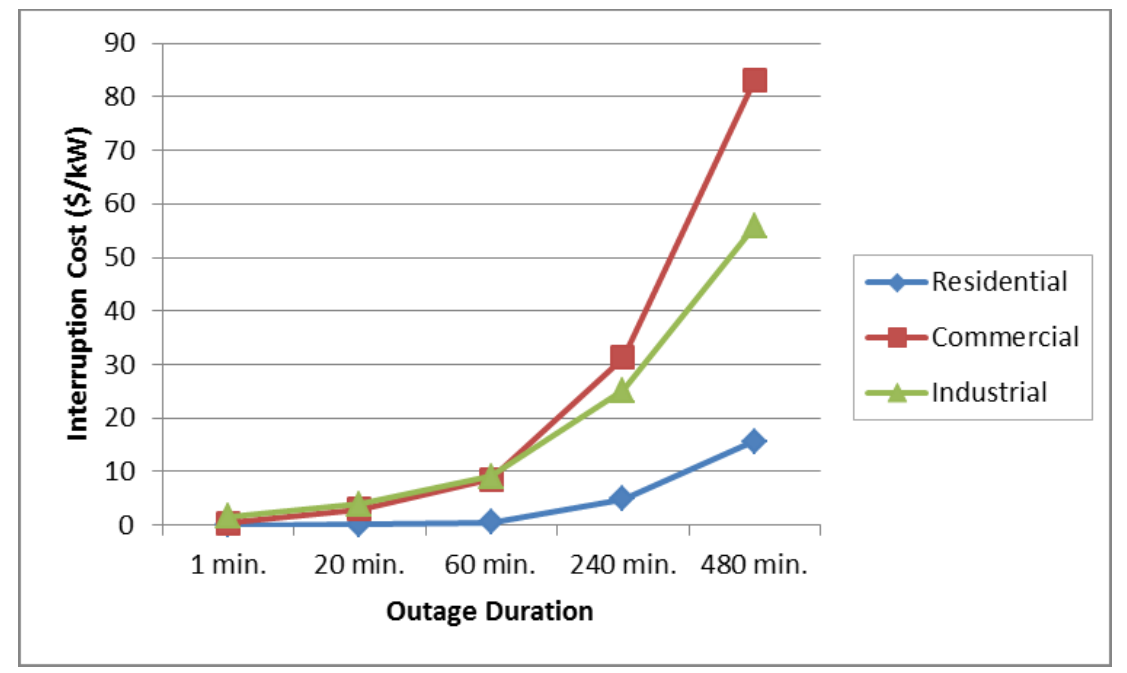

Figure 5-7: Customer Damage Functions

\subsubsection{Results}

Figure 5-8 and Table 5-4 furnish the optimal switch number and placement results for IEEE 34bus, 123-bus, WR\#3 and WR\#4 test systems. Table 5-4 lists least normalized customer interruption cost index $\mathrm{CICmi}$ and relative reduction in normalized customer cost interruption index CICDmi for number of switches from "0" to "6". Although all the indices shown in Table 5-4 are not necessarily required for the determination of optimal switch number and placement, these are shown to illustrate the working mechanism of the proposed algorithm. The iterative algorithm 
stops as soon as the CICDmi index goes below the predefined threshold of " 8 ". The optimal number and placement solution for IEEE 34-bus system is "1" and "SS-4". The optimal number and placement solution for IEEE 123-bus system is "2" and "SS-2, SS-5". The optimal number and placement solution for WR\#3 feeder is " 3 " and "SS-6, SS-10, SS-13". The optimal number and placement solution for WR\#4 feeder is "3" and "SS-5, SS-7, SS-11". Increasing the number of switches beyond the optimal number found here would return minimal reduction in total customer interruption cost as shown in Figure 5-8 and not worth exploring.

As per the solution provided by the proposed algorithm, installing one sectionalizing switch at SS4 on IEEE 34 -bus test feeder would reduce the total interruption cost by $69.9 \%$ compared to the base case with no switches. Similarly, installing three sectionalizing switches at SS-6, SS-10, and SS-13 on WR\#3 feeder would reduce the total interruption cost by $74.7 \%$ compared to the base case. Increasing the number of sectionalizing switches beyond the optimal number would result in relative reduction of interruption costs less than 8\%. Figure 5-3, Figure 5-4, and Figure 5-5 mark optimal placement of switches for IEEE 34-bus, IEEE-123 bus, WR\#3, and WR\#4 feeders respectively.

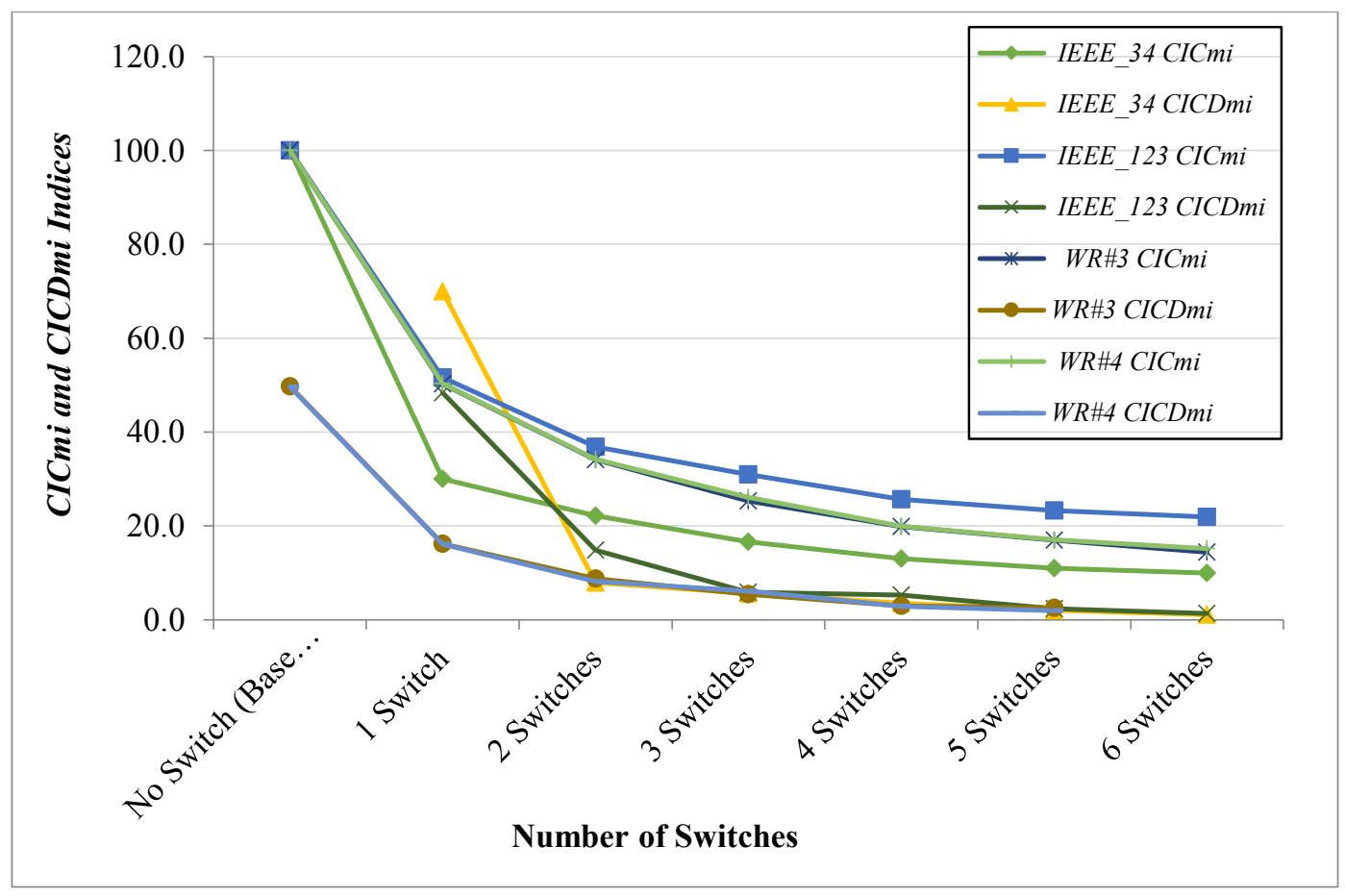

Figure 5-8: Calculated Indices for IEEE 34-Bus, IEEE-123 Bus, WR\#3, and WR\#4 Feeders 
Table 5-4: Calculated Indices for IEEE 34-Bus, IEEE-123 Bus, WR\#3, and WR\#4 Feeders

\begin{tabular}{|c|c|c|c|c|c|c|c|c|c|c|c|c|}
\hline & \multicolumn{3}{|c|}{ IEEE 34-Bus Feeder } & \multicolumn{3}{|c|}{ IEEE 123-Bus Feeder } & \multicolumn{3}{|c|}{ WR\#3 Feeder } & \multicolumn{3}{|c|}{ WR\#4 Feeder } \\
\hline $\begin{array}{l}\text { \# of } \\
\text { SWs }\end{array}$ & CICmi & $\begin{array}{l}C I C \\
D m i\end{array}$ & $\begin{array}{c}\text { Optimal } \\
\text { Switch } \\
\text { Location }\end{array}$ & CICmi & $\underset{m i}{C I C D}$ & $\begin{array}{l}\text { Optimal } \\
\text { Switch } \\
\text { Location }\end{array}$ & $\mathrm{CICmi}$ & $\underset{m i}{C I C D}$ & $\begin{array}{l}\text { Optimal } \\
\text { Switch } \\
\text { Location }\end{array}$ & CICmi & $\underset{m i}{C I C D}$ & $\begin{array}{l}\text { Optimal } \\
\text { Switch } \\
\text { Location }\end{array}$ \\
\hline 0 & 100.0 & N/A & N/A & 100.0 & N/A & N/A & 100.0 & N/A & N/A & 100.0 & N/A & N/A \\
\hline 1 & 30.1 & 69.9 & SS4* & 51.6 & 48.4 & SS3 & 50.3 & 49.7 & SS9 & 50.3 & 49.7 & SS8 \\
\hline 2 & 22.2 & 7.9 & $\mathrm{SS} 2, \mathrm{SS} 4$ & 36.8 & 14.8 & SS2, SS5* & 34.1 & 16.2 & SS6, SS11 & 34.2 & 16.1 & $\begin{array}{l}\text { SS6, } \\
\text { SS11 } \\
\end{array}$ \\
\hline 3 & 16.6 & 5.6 & $\begin{array}{l}\text { SS2, SS4, } \\
\text { SS5 }\end{array}$ & 30.9 & 5.9 & $\begin{array}{l}\text { SS2, SS4, } \\
\text { SS5 }\end{array}$ & 25.3 & 8.8 & $\begin{array}{c}\text { SS6, } \\
\text { SS10, } \\
\text { SS13* }\end{array}$ & 26.1 & 8.1 & $\begin{array}{l}\text { SS5, SS7, } \\
\text { SS11* }\end{array}$ \\
\hline 4 & 13.0 & 3.5 & $\begin{array}{l}\text { SS2, SS3, } \\
\text { SS4, SS5 }\end{array}$ & 25.6 & 5.3 & $\begin{array}{l}\text { SS1, SS2, } \\
\text { SS4, SS5 }\end{array}$ & 19.9 & 5.4 & $\begin{array}{c}\text { SS5, SS7, } \\
\text { SS11, } \\
\text { SS13 }\end{array}$ & 19.9 & 6.1 & $\begin{array}{c}\text { SS5, SS6, } \\
\text { SS10, } \\
\text { SS12 }\end{array}$ \\
\hline 5 & 11.0 & 2.0 & $\begin{array}{l}\text { SS2, SS3, } \\
\text { SS4, SS5 } \\
\text { SS6 }\end{array}$ & 23.3 & 2.4 & $\begin{array}{l}\text { SS1, SS2, } \\
\text { SS4, SS5 } \\
\text { SS6 }\end{array}$ & 16.9 & 3.0 & $\begin{array}{c}\text { SS4, SS6, } \\
\text { SS9, } \\
\text { SS11, } \\
\text { SS13 }\end{array}$ & 17.0 & 2.9 & $\begin{array}{c}\text { SS5, SS6, } \\
\text { SS9, } \\
\text { SS11, } \\
\text { SS13 }\end{array}$ \\
\hline 6 & 9.9 & 1.1 & $\begin{array}{l}\text { SS1, SS2, } \\
\text { SS3, SS4, } \\
\text { SS5, SS6 }\end{array}$ & 21.9 & 1.3 & $\begin{array}{l}\text { SS1, SS2, } \\
\text { SS4, SS5, } \\
\text { SS6, SS7 }\end{array}$ & 14.4 & 2.5 & $\begin{array}{c}\text { SS4, SS6, } \\
\text { SS8, } \\
\text { SS10, } \\
\text { SS12, } \\
\text { SS14 }\end{array}$ & 15.2 & 1.9 & $\begin{array}{c}\text { SS4, SS5, } \\
\text { SS6, SS9, } \\
\text { SS11, } \\
\text { SS13 }\end{array}$ \\
\hline
\end{tabular}

* Optimal switch location determined by the proposed iterative algorithm.

The number of $C I C$ computations increases as the number of switches " $r$ " value increases. The " $r "=1$ scenario costs the least processing time as it requires the least number of $C I C$ computations compared to other " $r$ " $>1$ scenarios. Therefore, the proposed iterative algorithm starts with " $r$ " $=1$ scenario and stops as soon as the desired solution is achieved. This strategy helps reducing the search space of the problem. According to equation (5.3), there would be 63, 127, 32767, and 16383 possible combinations of switch placements on IEEE 34-bus, IEEE-123 bus, WR\#3, and WR\#4 feeders respectively. The customer interruption cost $C I C$ needs to be computed for all the possible combinations to arrive at a global optimal solution. However, the proposed approach cuts down the search space drastically to $21,63,1940$, and 1470 possible combinations for these test systems and arrives at an optimal solution that satisfies the defined criteria for minimum reduction in customer interruption costs. This greatly simplifies computational complexity of the problem and shows efficacy of the proposed solution. Figure 5-9 shows the comparison of search space in terms of number of $C I C$ computations needed for traditional switch optimization approach [67] and the proposed iterative algorithm. 


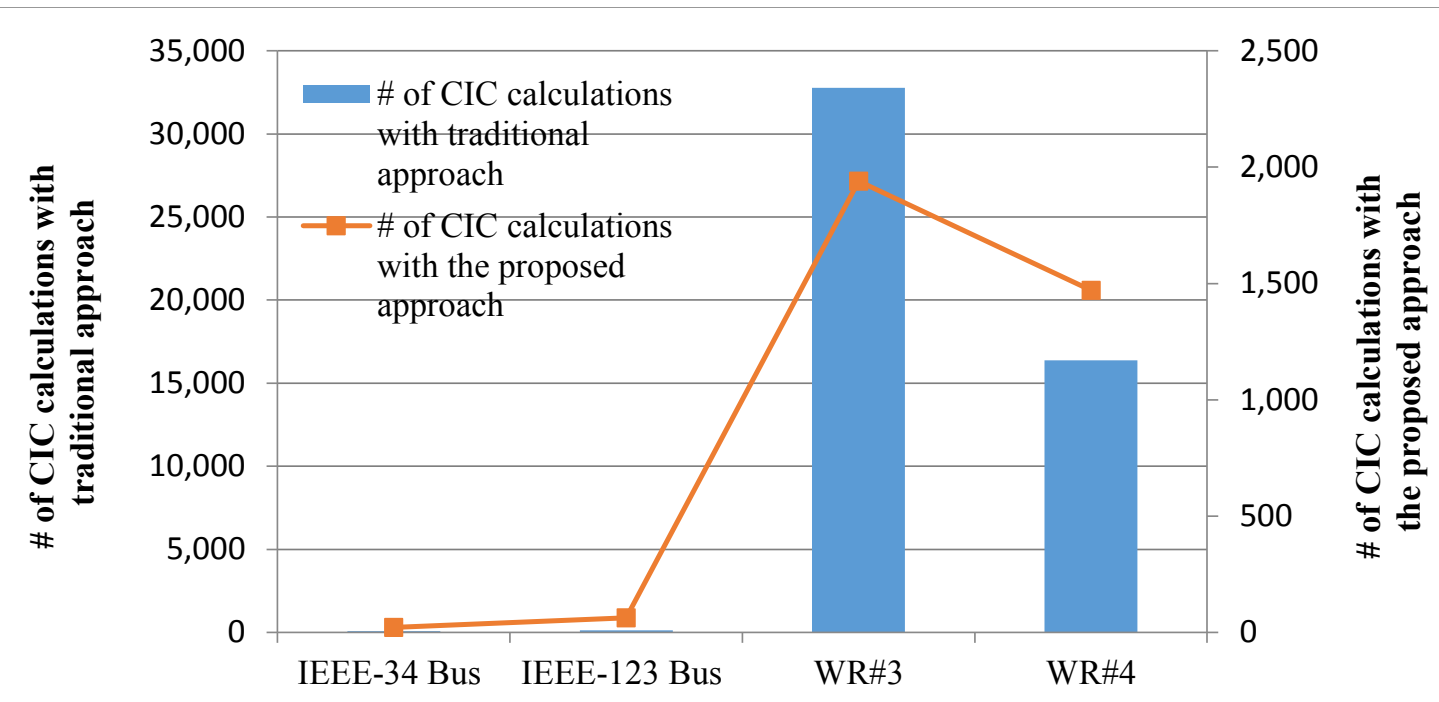

Test Feeder

Figure 5-9: Comparison of CIC Calculations between Traditional [67] and Proposed Approaches 


\section{Chapter 6}

\section{DG Fault Characteristics}

The smart grid initiative is the main cause of increasing DG penetration in electrical distribution system. As the DG systems become prevalent, the existing protection philosophies get impacted and warrants for a closer investigation of fault response of various types of DG resources and their impacts to the existing protection philosophies. The DG resources can be broadly classified into inverter based and non-inverter based resources. The inverter based sources include photovoltaic, micro-turbines, fuel cells, and wind turbines with Doubly Fed Induction Generators (DFIG). On the other hand non-inverter based resources include synchronous and induction generators.

Unlike the non-inverter based DG resources, the inverter based DG resources supply limited fault currents that could lead to malfunctioning of the distribution network protection system which largely relies on overcurrent based protection techniques. The absence of mathematical models and methods to calculate the inverter fault current contribution has been a significant challenge to the introduction of inverter based DGs [69]. The inverters are actively current limited due to the low thermal inertia of semiconductor switches, and thus have a small fault current contribution (1$2 \mathrm{pu}$ ) compared to conventional non-inverter based generators (5-10 pu). 


\subsection{Short Circuit Analysis}

Short-circuit studies involve calculating available fault levels at various points in the system to ensure that the protective devices employed in the system are sufficiently sized to interrupt or withstand short-circuit current. There are several types of faults that can occur on the EDS. The most common type of fault is single-line-to-ground fault which occurs when one of the phases comes into contact with alternative current path or ground. A 3-phase fault occurs when all three phases come into contact with each other and is the least common type of fault. The 3-phase fault current typically provides the highest available fault current. However, there are situations where this is not the case.

A fault in a typical EDS behaves very much like a Resistive-Inductive (RL) circuit as shown in Figure 6-1 with the switch in the closed position. Closing the switch simulates a faulted condition.

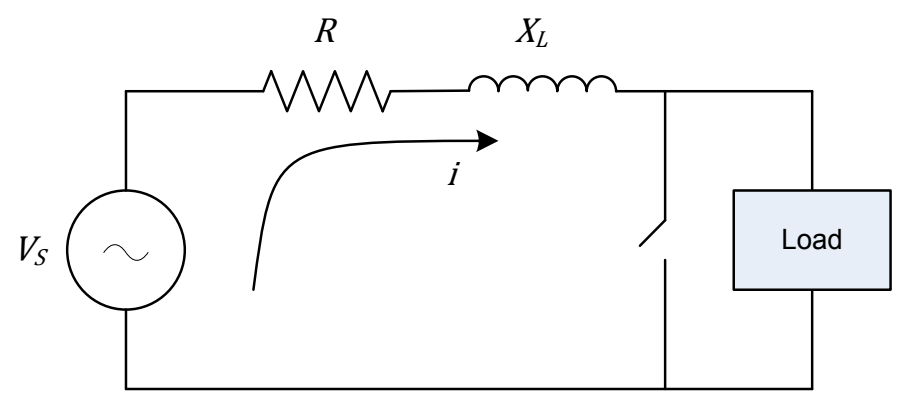

Figure 6-1: Circuit Model for Asymmetrical Fault Current

The total fault current ignoring initial value of fault current is given by equation (6.1) which includes AC current plus the DC offset current. A representation of fault current response is given in Figure 6-2.

$$
i_{f}(t)=i_{a c}(t)+i_{d c}(t)=\frac{\sqrt{2} V}{Z}\left[\sin (\omega t+\alpha-\theta)-\sin (\alpha-\theta) e^{-\left(\frac{t}{T}\right)}\right]
$$

Where $Z=\sqrt{R^{2}+\mathrm{X}_{\mathrm{L}}^{2}}, \mathrm{~V}_{\mathrm{S}}=\sqrt{2} V \sin (\omega \mathrm{t}+\alpha), \theta=\tan ^{-1}\left(\mathrm{X}_{\mathrm{L}} / R\right), \omega$ is angular frequency, and $\mathrm{T}$ is the time constant defined by equation (6.2). 


$$
T=\frac{X_{L}}{\omega R}
$$

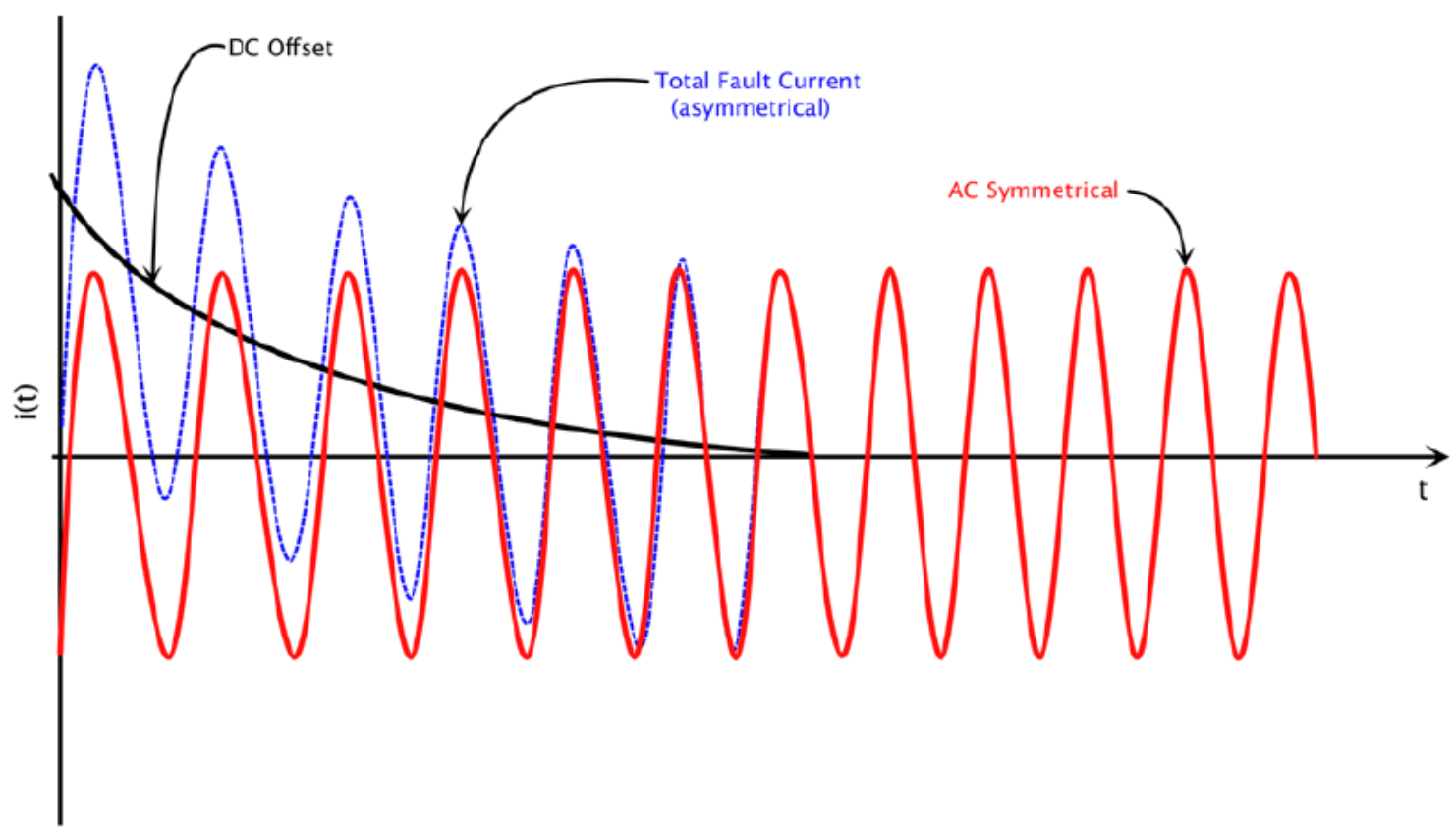

Figure 6-2: Short Circuit Asymmetrical Current [70]

\subsection{Synchronous Machine Fault Response}

The short circuit response of a synchronous generator for a fault at its terminal is a decaying pattern that settles to steady state value after some time. Synchronous machines generally deliver about six times the rated current for several cycles before decaying to between $400 \%$ and $200 \%$ of rated current [71] [72] as shown in Figure 6-3. The external excitation system continues to provide voltage to the field winding of the generator. The prime mover continues to drive the rotor that produces the required induced voltage in the stator winding which in turn supplies a continuous fault current. The steady state fault current value will persist unless interrupted by a protective device such as a circuit breaker.

The industry has established three reactance variables called sub-transient, transient, and synchronous reactance [73] to model the short circuit behavior synchronous machines. Sub- 
transient reactance $\left(\mathrm{X}_{\mathrm{d}}^{\prime \prime}\right)$ determines current during first cycle after fault occurs. This condition lasts for approximately 0.1 seconds. Transient reactance $\left(\mathrm{X}_{\mathrm{d}}^{\prime}\right)$ determines current after several cycles. This condition lasts from 0.5 to 2 seconds. Synchronous reactance $\left(X_{d}\right)$ determines steady state fault current.

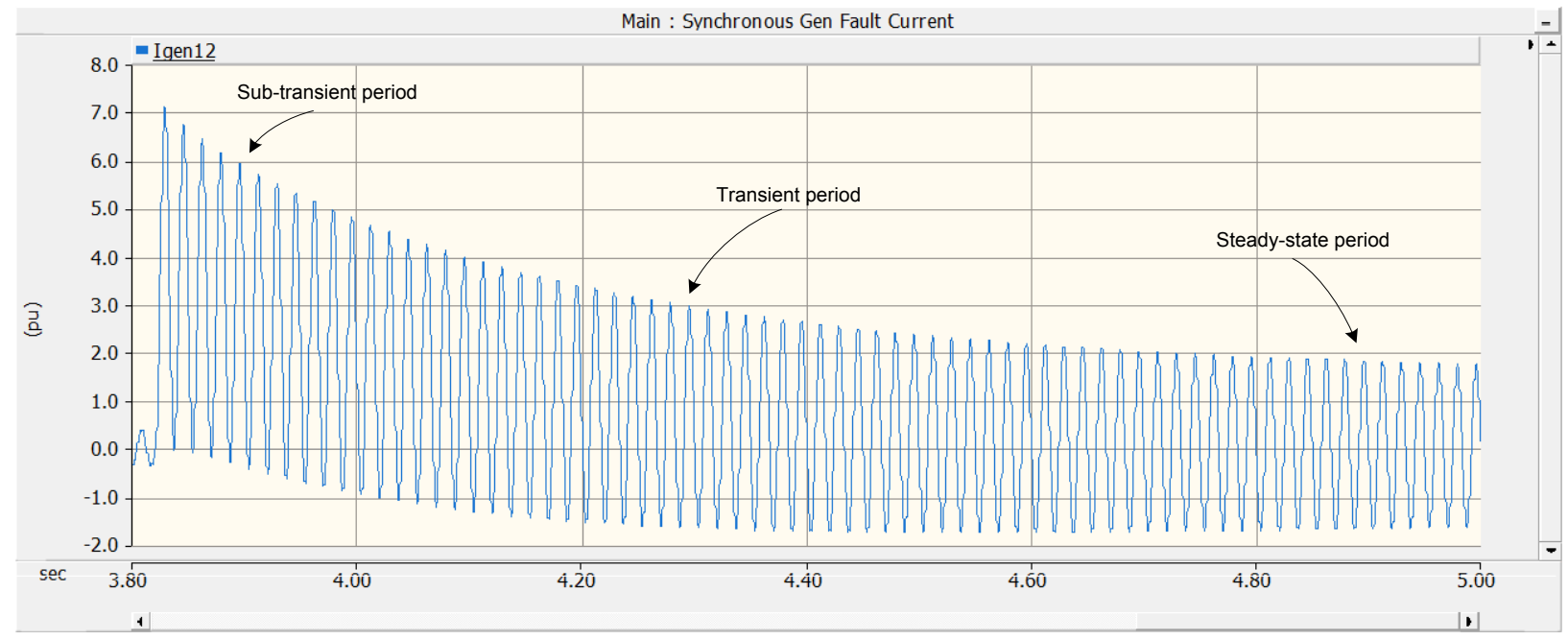

Figure 6-3: Synchronous Machine Response to Three Phase Fault

\subsection{Induction Machine Fault Response}

If a short circuit is applied at the terminal of an induction machine, the current will start out very high before decaying completely. The induction machines deliver about six times rated current during this time as shown in Figure 6-4 [72] [73] [74]. Unlike the synchronous machine the fault characteristic is generated by inertia driving the motor in the presence of the field flux produced by induction from the stator. During the fault the flux decays due to loss of induction and thus the fault current decays to zero [73].

The values of transient and synchronous reactance approach infinity under steady-state fault conditions. Therefore, the induction motors are assigned only a sub-transient value of reactance. This value varies upward from the locked rotor reactance to account for the decay of the motor current contribution to the fault. For fault calculations, an induction generator can be treated the same as induction motor. Wound-rotor induction motors normally operating with their rotor rings 
short-circuited will contribute short-circuit current in the same manner as a squirrel-cage induction motor [73].

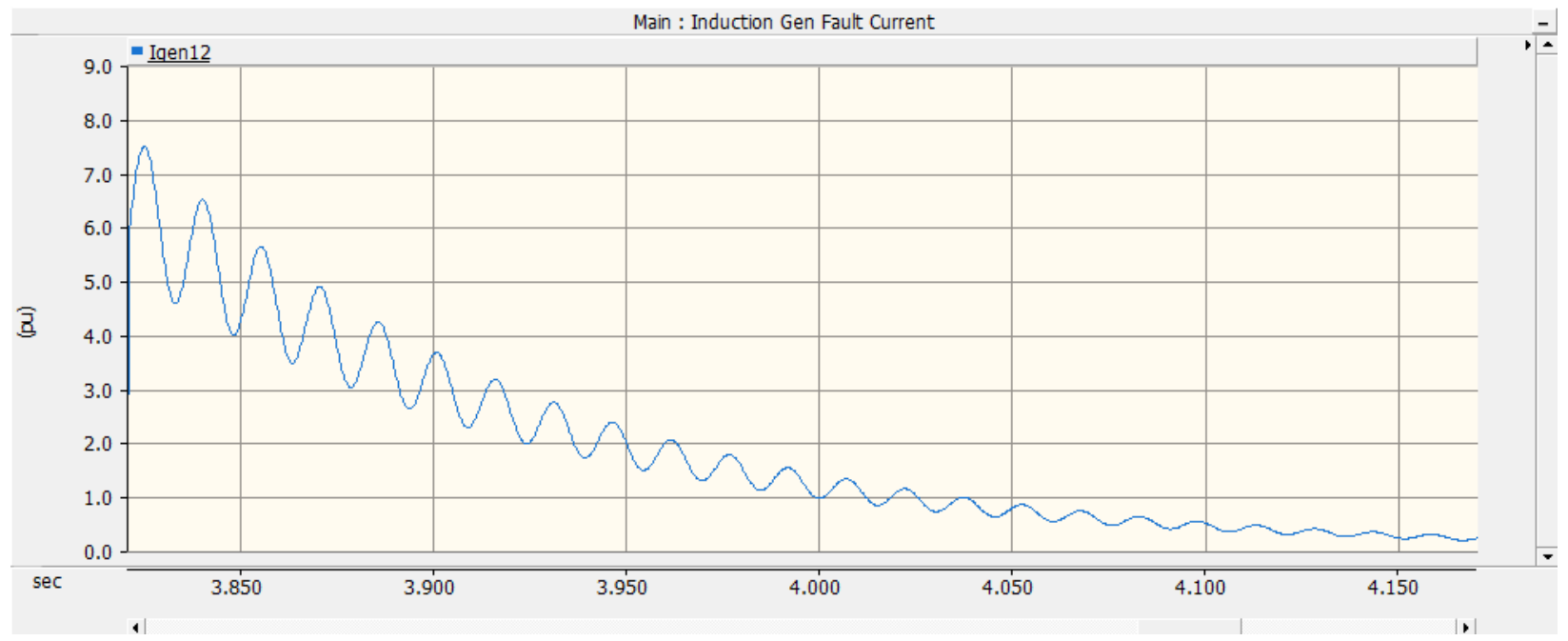

Figure 6-4: Induction Machine Response to Three Phase Fault

\subsection{Inverter based DG Fault Response}

Inverter based DGs lack rotating mass that is present with conventional non-inverter based resources, and they behave dynamically different compared to synchronous or induction machines. Inverters do not develop inertia to carry fault current based on an electro-magnetic characteristic. Power electronic inverters have a much faster decaying envelope for fault currents because the devices lack predominately inductive characteristics that are associated with rotating machines. The fault response of an inverter based DG depends mostly on its control system rather than its physical parameters. Moreover, depending on its method of current limiting, an inverter's fault response depends on whether the fault is symmetric or asymmetric [75].

\subsubsection{Inverter based DG}

Figure 6-5 gives a typical representation of an inverter based DG. It consists of DC Power Generating Unit, DC-DC converter, DC link capacitor, DC-AC inverter, and a LCL filter for grid interconnection. The DC Power Generating Unit could be a photovoltaic system, fuel cell, micro- 
turbine, or an energy storage system. The inverter converts the DC voltage into three-phase ac voltage. The inverter system usually consists a controller that controls the output complex power of the inverter and ensures the output ac voltage is synchronized with the electric grid voltage. The DC link capacitor holds the dc output voltage constant during short transients. Therefore, during transients such as faulted conditions the inverter response mainly depends on the controller [71].

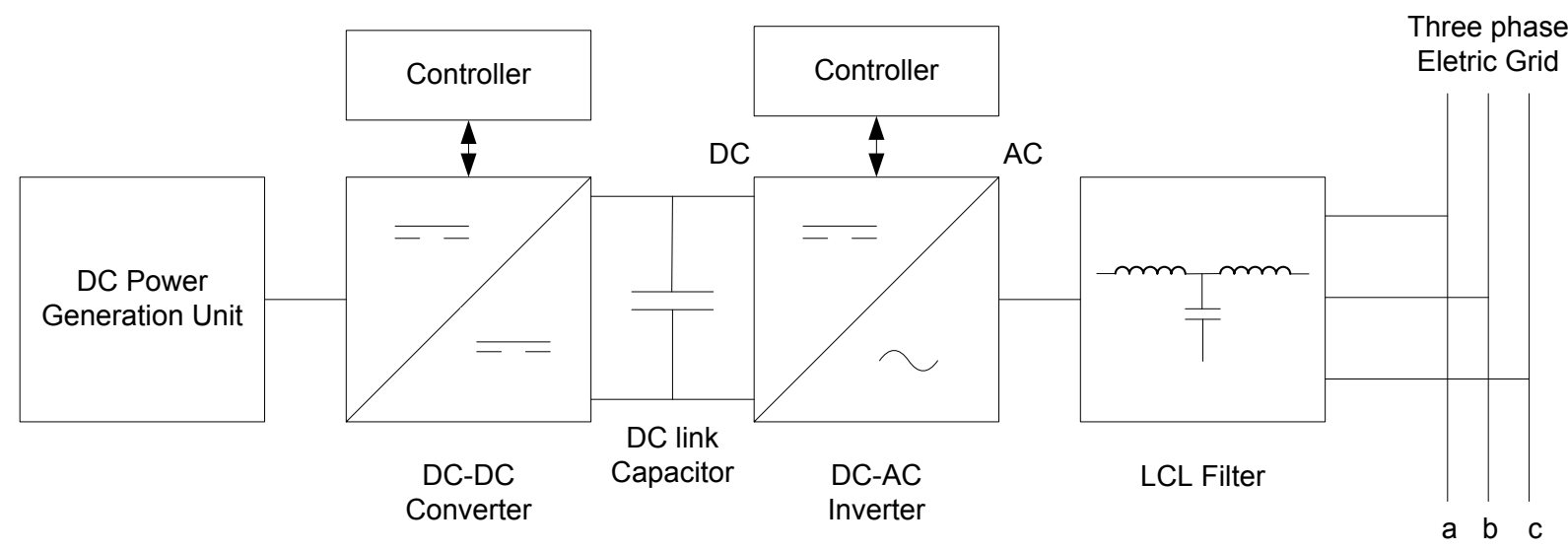

Figure 6-5: Inverter based DG

\subsubsection{Inverter Control}

In order to control the real and reactive power output of inverter, there are two main methods exist. The first method is known as voltage-mode control and the second method is current-mode control which is also referred to as multi-loop control.

\subsubsection{Inverter Voltage-mode Control}

Figure 6-6 shows the voltage-mode control of inverter. The real and reactive power are controlled, respectively, by phase angle and amplitude of the inverter voltage relative to the ac grid voltage. Therefore, the voltage controlled equivalent circuit shown in Figure 6-6 can be used to represent the inverter based DG during the transient period for this control method [76] [77]. As indicated in the figure, simple PI controllers can be used to regulate the output power in the voltage-mode control scheme. The main shortcoming of the voltage-mode control is that there is no control loop 
on the inverter line current. Therefore, the inverter is not protected against overcurrent situations, and the current may undergo large excursions if the power commands are rapidly changed or faults are taken place in the ac electric grid.

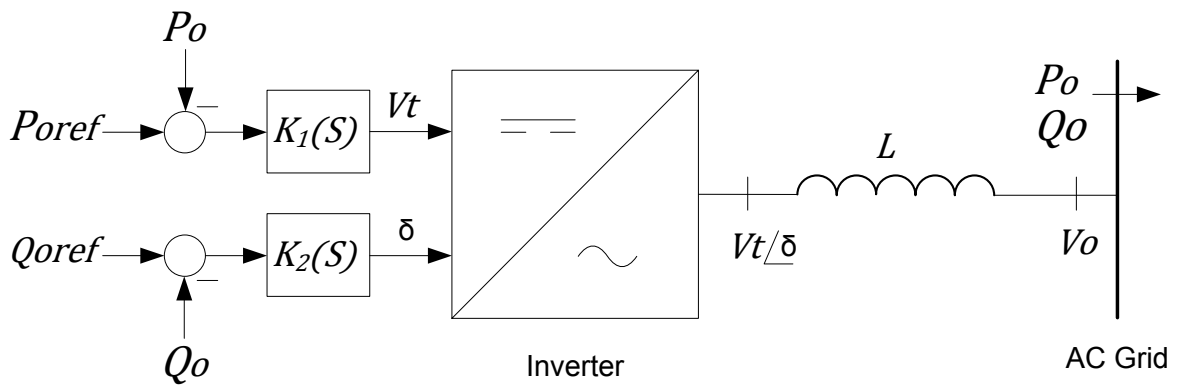

Figure 6-6: Grid-connected Inverter Voltage-mode Control

\subsubsection{Inverter Current-mode Control}

The current-mode control scheme has two control loops namely current control loop and power control loop. The inner current control loop regulates the output current of inverter based DG, and outer power controller regulates the power output. The current loop is much faster in comparison with the power control loop. The power controller determines the reference current signal for the current controller. Figure 6-7 shows the typical representation of inverter current-control scheme provided in [75]. This setup comprises a three-phase three-leg inverter bridge, a LCL-filter, and a power controller, an inductor current control loop, a grid synchronization module or a Phase Locked Loop (PLL), and a current limiting module.

This method of control has an advantage over the voltage-mode control as the current regulation scheme ensures that the inverter is protected against overcurrent conditions. Other advantages of the current-mode control include robustness against variations in parameters of the inverter system and the ac system, superior dynamic performance, and higher control precision. Due to these benefits, this controls scheme is widely used in most of the inverter based DG applications. 


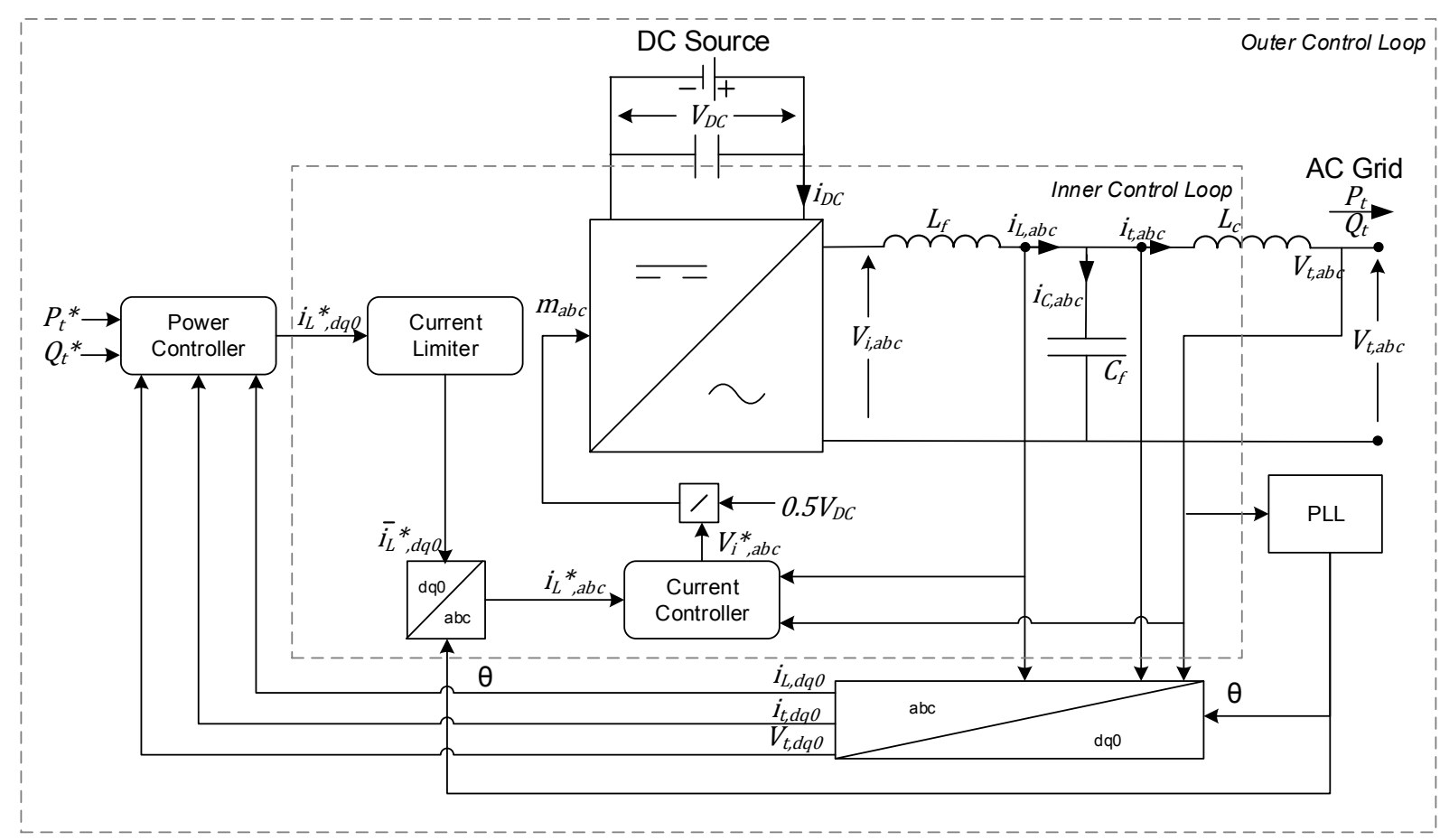

Figure 6-7: Grid-connected Inverter Current-mode Control

The outer power control loop regulates the output power to given setpoint $S_{t, r e f}=P_{t, r e f}+$ $j Q_{t, r e f}$. According to the instantaneous power theory, the output current references required to maintain the set output power $\left(P_{t, r e f}\right.$ and $\left.Q_{t, r e f}\right)$ and an output voltage of $\left(v_{t d}, v_{t q}\right)$ is given by equation (6.3).

$$
\left[\begin{array}{l}
i_{t d, r e f} \\
i_{t q, \text { ref }}
\end{array}\right]=\frac{1}{v_{t d}^{2}+v_{t q}^{2}} *\left[\begin{array}{cc}
v_{t d} & v_{t q} \\
v_{t q} & -v_{t d}
\end{array}\right] *\left[\begin{array}{l}
P_{t, r e f} \\
Q_{t, r e f}
\end{array}\right]
$$

The current control loop regulates the filter inductor current. As shown in Figure 6-7, $i_{C}=i_{L}-$ $i_{t}$, and $i_{L, r e f}=i_{t, r e f}+i_{C}$, the inductor reference currents can be found from,

$$
i_{L, r e f}=i_{t, r e f}+i_{L}-i_{t}
$$


A low pass filter as shown in Figure 6-8 is used to achieve instantaneous power control that can produce high inductor current quality. In steady state, the inductor current references are clean DC signals without any switching harmonics and double harmonic ripple due to unbalance. During unbalance condition, the grid voltage is characterized by positive, negative, and zero sequence components.

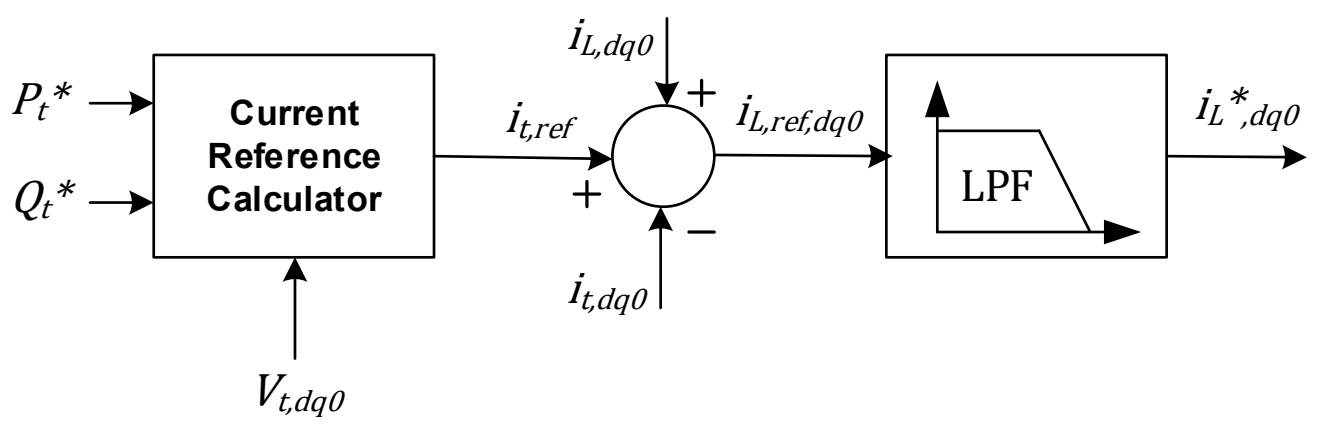

Figure 6-8: Power Controller Implementation

If the inverter configuration does not involve neutral wire, the current reference calculation in zero sequence is not needed. The negative sequence component of current reference appears as a $2 \omega$ component in a dq0 reference, and thus has a zero time average, and gets filtered out by the filter. Therefore, the current reference calculation in the inverter current control is based only on the positive sequence of the grid voltage $\left(v_{t p}\right)$ and hence it only generates a positive sequence current reference [75]. The inductor current reference to satisfy a given complex power reference is given in the following equation. The proposed calculation of the current reference depends on the ability of PLL to accurately estimate the phase angle information of the grid voltage positive sequence component.

$$
i_{L, r e f}=\operatorname{conj}\left(\frac{P_{t, r e f}+j Q_{t, r e f}}{3 * v_{t p}}\right)+v_{t p} * j \omega C_{f}
$$

The inverter exhibits rather low thermal inertia and they need protection against overcurrent which is achieved by current limiting. There are several approaches of current limiting. The simplest method is to implement hard saturation limits in natural reference frame (abc frame) to limit the 
instantaneous magnitude of the per-phase inductor current reference. This method results in clipping and distorted waveforms which can introduce power quality issues on the grid side. The second method involves implementing hard saturation limits in synchronously rotating frame (dq0 frame) when the direct and quadrature axis components exceed a pre-defined threshold. Since the current reference only possess positive sequence component there will be no $2 \omega$ sinusoidal ripple and no distortion.

The third method, referred to as latched limiting, is similar to the second method with one major difference where the inductor current is switched to a pre-defined fault current reference $\left(i_{f}\right)$ once the inductor current reference has exceeded the threshold. The last method serves more realistic applications where the grid interconnected DG units need to supply required amount of fault current to ensure proper protection of the EDS.

\subsubsection{Fault Characteristics and Model of Inverter}

According to [71], the inverter fault response under voltage-mode control has a high initial current overshoot and then the controller brings the current to steady state in a few cycles. Whereas the inverter under current-model control, the current increases much slower and then decreases back to the steady-state value rather slowly due to slower power control loop. However, the current in the current-mode control is well regulated and limited. For photovoltaic inverter based DG, the fault contribution is really limited due to the fact that these applications do not use the outer power control loop, or they are very slow [78]. Due to the popularity of the current-mode control scheme, the research work only focused on studying the inverter fault response under this control.

Faults on the electric grid are characterized by voltage drop at the fault location. When the fault happens, the power controller increases the inductor current reference to compensate for the drop in voltage and maintains the set power output. The power controller has a low pass filter with a cut off frequency of $\omega_{c}$, which makes the inductor current reference to increase in an exponential manner after a step change in the inverter output voltage at $t=t_{f}$ and is given by, 


$$
i_{L, r e f}(t)=i_{L, r e f}\left(t_{0}\right)+\left(i_{L, r e f}\left(t_{f}\right)-i_{L, r e f}\left(t_{0}\right)\right) *\left(1-e^{-\omega_{c} t}\right)
$$

The current limiting will be switched on when the magnitude of the current reference exceeds the predefined threshold $\left(i_{t h}\right)$. As per the latched limiting method the current reference will be switched to pre-defined fault current reference $\left(i_{f}\right)$. Usually, the limit is set close to 2 pu and it causes the fault contribution of the inverter to not exceed $2 \mathrm{pu}$. The transition of inductor current reference is given by equation (6.7). Figure 6-9 illustrates a typical fault response of the inverter.

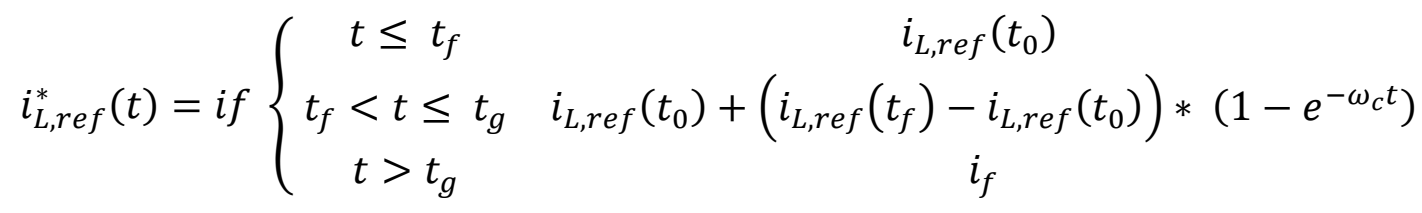

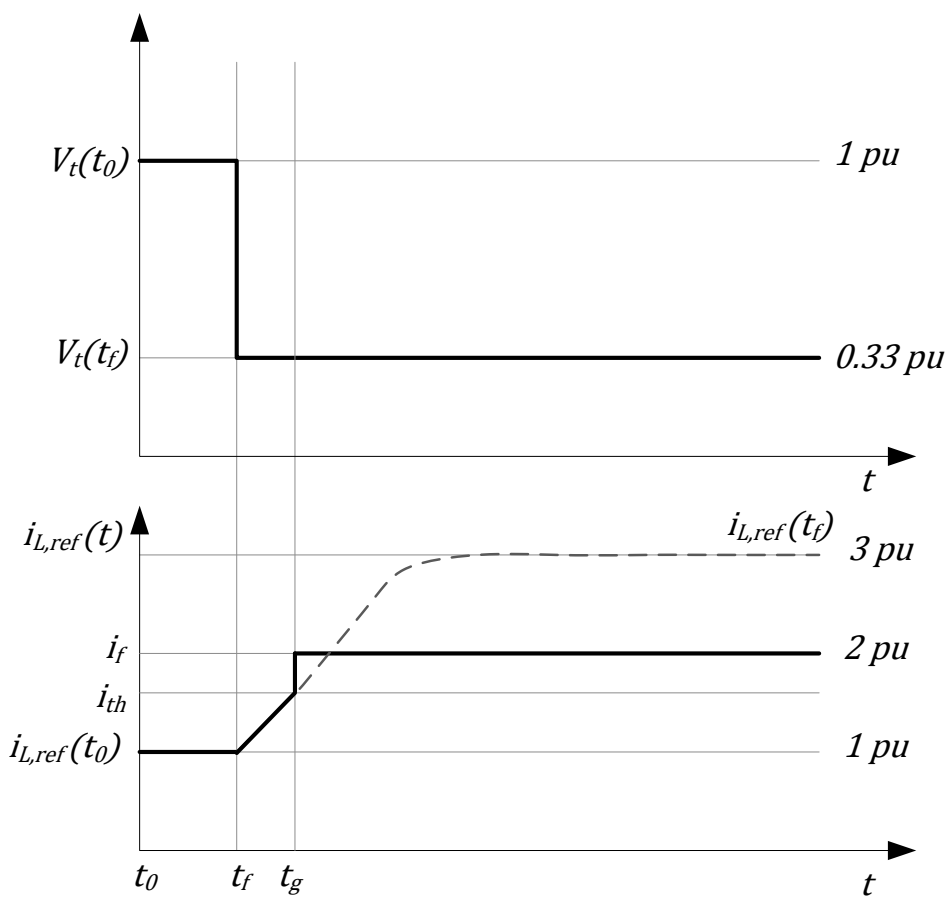

Figure 6-9: Fault Response of an Inverter under Current-mode Control

The fault characteristics of the inverter suggests that inverter with current limiting is equivalent to a controlled current source of $T_{c l}(s) * i_{f}$ with a parallel filter capacitor admittance $Y_{c f}$ as illustrated in Figure 6-10. $T_{c l}(s)$ is the closed loop transfer function of the control loop. During fault 
condition, the inverter behaves like a constant current source providing positive sequence current only. The inverter model in negative and zero sequence has no active sources, and only contain the parallel capacitor admittance. Figure 6-11 illustrates equivalent circuits of grid-connected inverter in positivize, negative, and zero sequence. It is important to note that the negative sequence component of the grid voltage can drive currents through the filter capacitor.

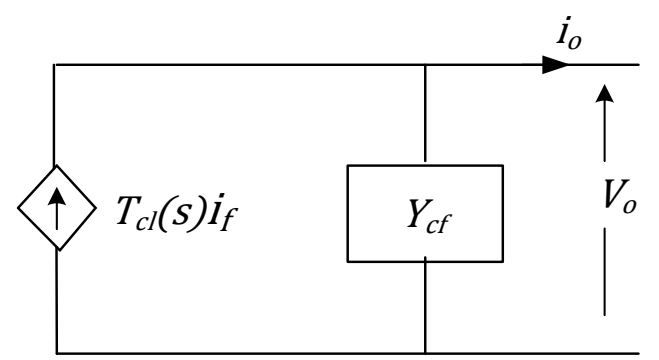

Figure 6-10: Equivalent Circuit of Grid-connected Inverter with Current Limiting
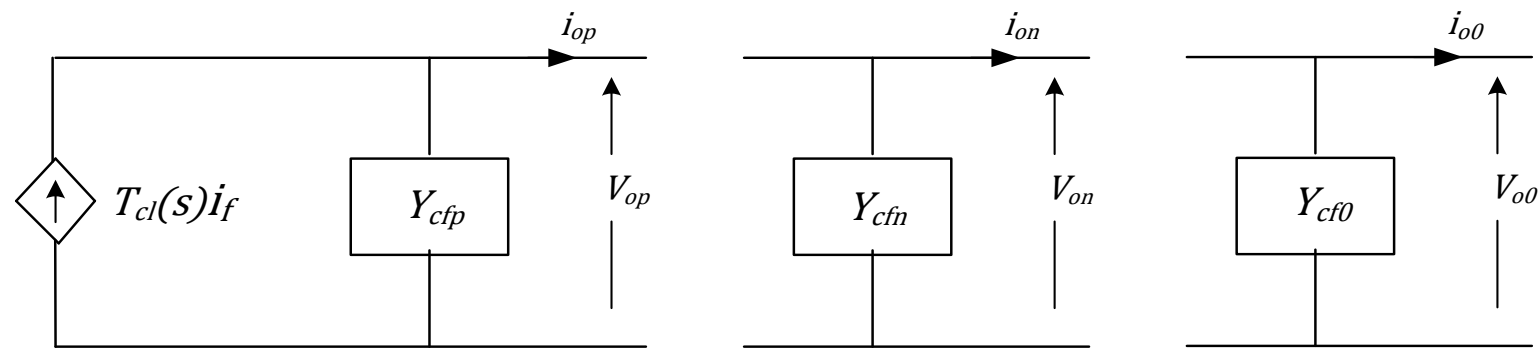

Figure 6-11: Equivalent Sequence Network of Grid-connected Inverter 


\section{Chapter 7}

\section{MAS Fault Location \& Isolation}

SSAs continuously monitor the voltage and current phasor data at their node and neighboring nodes, and employ Fault Detection Algorithm explained in the following section to autonomously determine the occurrence of a permanent fault downstream. The SSAs set Permanent Fault Flag $\left(P F_{f}\right)$ status to TRUE when there is a fault downstream. In general, the fault current flows from source to the fault point when a fault occurs on a radial distribution feeder with no distributed generation interconnected. However, when EDS has multiple DG interconnections the fault current is supplied from all these sources, and the fault magnitudes are based on their individual fault characteristics. Following the fault theory, the fault point always has the deepest voltage sag compared to the rest of the network and the zone it is associated with sees highest change in the current flow. This underlying principle is executed by the agent communications to locate the fault zone of the feeder.

Fault location starts when the feeder recloser or circuit breaker is locked out on a permanent fault and FA issues the command to locate the fault to its downstream SSAs. All SSAs check if the $P F_{f}$ status is TRUE at their node. If the $S S A_{i}$ at node- $i$ identifies that its $P F_{f}$ status is TRUE, then $S S A_{i}$ determines that the fault is located between its node- $i$ and downstream node $(i+1)$. Once a SSA finds the fault location, it communicates the fault location to its respective FA. This SSA is also responsible for fault isolation to make sure the SSWs on both ends of the faulty zone are open. To achieve this, the SSA sends a control signal to its SSW to open-up and lock in that position. It also communicates to its downstream SSA to open-up the downstream SSW. This ensures safety of 
utility crew when they repair the fault in the system. Once the SSA isolates the fault zone, it communicates to the respective FA indicating to start the service restoration process. The flow chart of agent communications responsible for the fault location and isolation is shown in Figure 7-1.

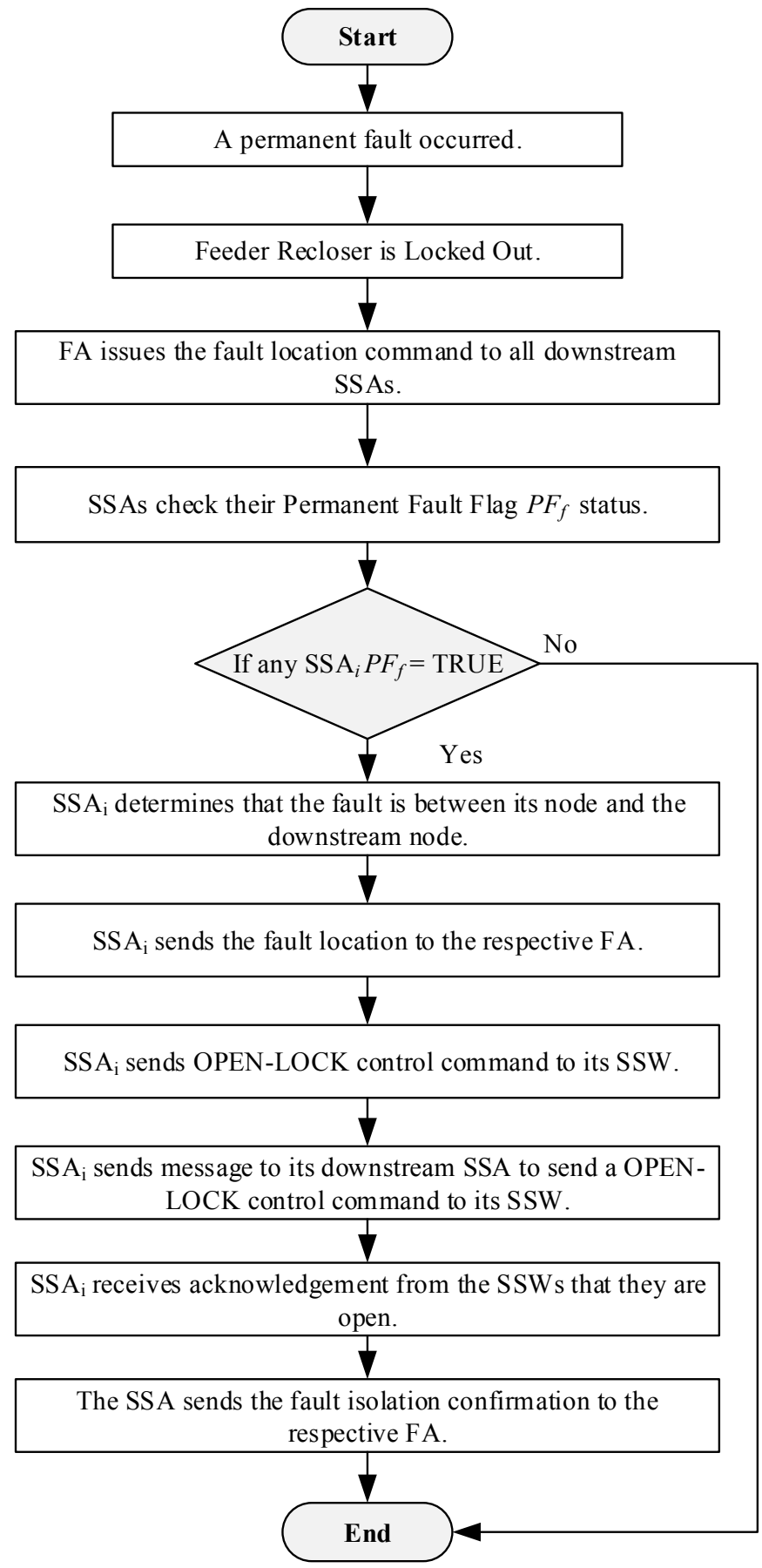

Figure 7-1: Agent Communication for Fault Location and Isolation 


\subsection{Fault Detection Algorithm}

The fault detection algorithm sets the permanent fault flag when it detects a permanent over current fault. The algorithm consists of four major blocks,

- Loss of Voltage (LOV) Detection

- Zone Over Current (ZC) Detection

- Over Current (OC) Fault Detection

- Permanent Fault (PF) Determination

As mentioned above all SSA have access to voltage and current phasor data of its local node. And through communications they can gather voltage and current phasor information from their neighboring agents. The SSA captures high fidelity oscillography data for events whenever the voltage and current quantities go above the programmed thresholds.

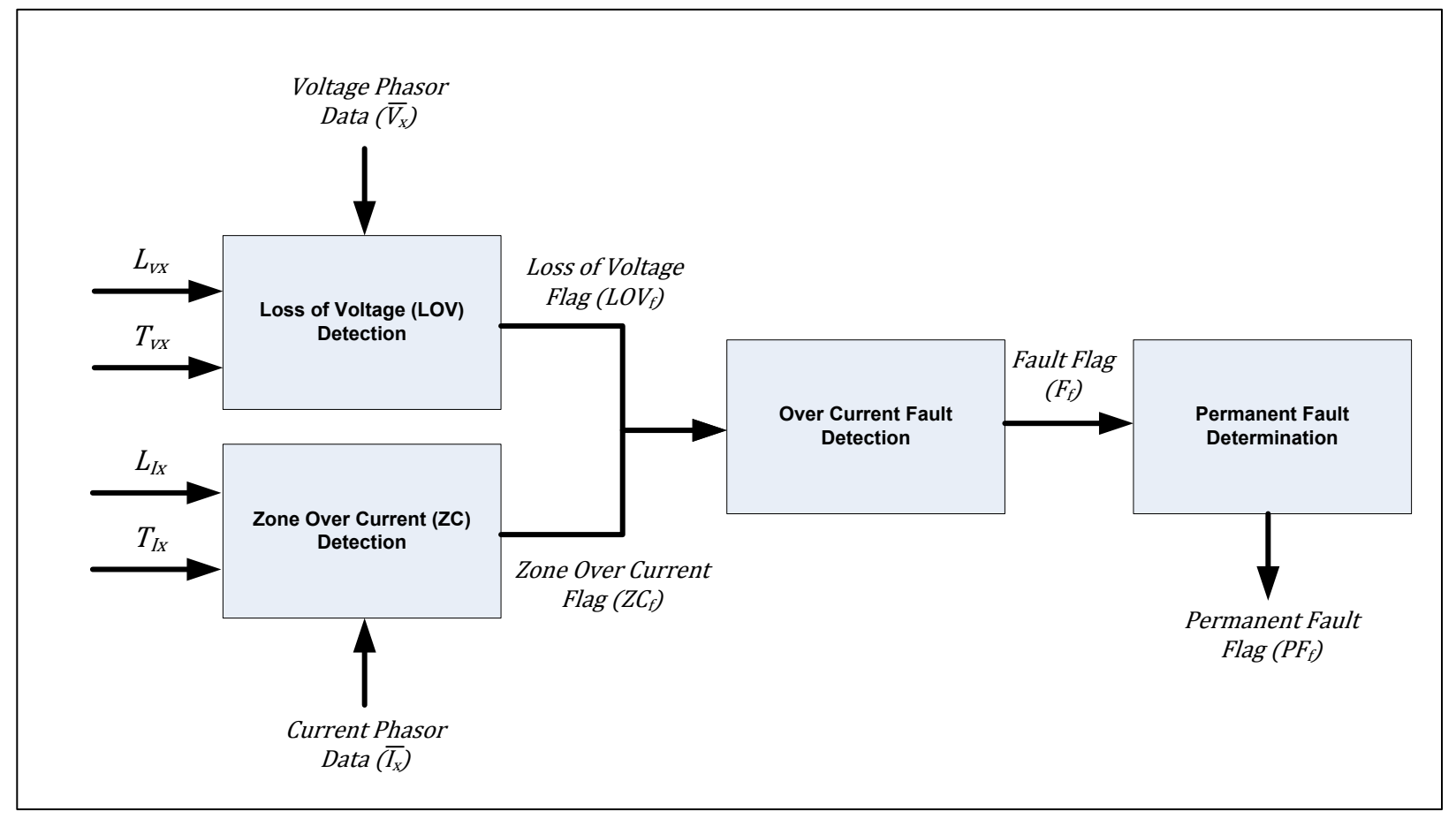

Figure 7-2: Fault Detection Algorithm Blocks 


\subsubsection{Loss of Voltage (LOV) Detection}

This block sets the LOV flag $\left(\operatorname{LOV}_{f}\right)$ of phase- $x$ when the rms magnitude of phase to ground voltage $v_{x}$ is below the pre-defined voltage level $L_{v x}$ for a specified amount of time $T_{v x}$. This detection is done on a per phase basis and " $x$ " denotes the respective phase " $a ", " b "$, and "c". The chart in Figure 7-3 shows the LOV detection logic. Although the values of $L_{v x}$ and $T_{v x}$ are configurable, the algorithm uses typical values of 0.2 pu and 2 cycles respectively.

\subsubsection{Zone Over Current (ZC) Detection}

This stage of the algorithm calculates the zone current value at each SSA node as indicated in equation (7.3). The zone current computation at a specific SSA node requires current measurements from all the DGs present in the zone and from the downstream SSA.

$$
\begin{gathered}
I_{Z_{x}^{i}}=\sum_{j=1}^{p} I_{E_{x}^{j}}-\sum_{j=1}^{q} I_{D_{x}^{j}} \\
I_{Z_{x}^{i}}= \pm I_{S S A_{x}^{i+1}}+\sum_{k=1}^{n} I_{D G_{x}^{k}} \pm I_{S S A_{x}^{i+2}} \\
I_{Z_{x}^{i}}=i f \begin{cases}\left|v_{x}^{i}\right|>\left|v_{x}^{i+1}\right|>\left|v_{x}^{i+2}\right| ; & I_{S S A_{x}^{i+1}}+\sum_{k=1}^{n} I_{D G_{x}^{k}}-I_{S S A_{x}^{i+2}} \\
\left|v_{x}^{i}\right|<\left|v_{x}^{i+1}\right|<\left|v_{x}^{i+2}\right| ; & -I_{S S A_{x}^{i+1}}+\sum_{k=1}^{n} I_{D G_{x}^{k}}+I_{S S A_{x}^{i+2}} \\
\left|v_{x}^{i}\right|>\left|v_{x}^{i+1}\right|<\left|v_{x}^{i+2}\right| ; & I_{S S A_{x}^{i+1}}+\sum_{k=1}^{n} I_{D G_{x}^{k}}+I_{S S A_{x}^{i+2}}\end{cases}
\end{gathered}
$$

Where,

$I_{Z_{x}^{i}}$

$I_{E_{x}^{k}}$

$I_{D_{x}^{k}}$ $S S W_{i+1}$ phase- $x$ rms current magnitude of zone- $i$ phase- $x$ rms current magnitude of entering element $k$ of zone- $i$ phase- $x$ rms current magnitude of departing element $-k$ of zone- $i$ upstream SSW of zone- $i$ 


$\begin{array}{ll}S S W_{i+2} & \text { downstream SSW of zone- } i \\ I_{S S W_{x}^{i+1}} & \text { phase- } x \text { rms current magnitude measured at } S S W_{i+1} \\ I_{S S W_{x}^{i+2}} & \text { phase- } x \text { rms current magnitude measured at } S S W_{i+2} \\ I_{D G_{x}^{k}} & \text { phase- } x \text { rms current magnitude measured at } D G^{k} \text { location } \\ n & \text { number of DGs present in zone- } i \\ p & \text { number of current entering elements present in zone- } i \\ q & \text { number of current departing elements present in zone- } i \\ \left|v_{x}^{i}\right|,\left|v_{x}^{i+1}\right|,\left|v_{x}^{i+2}\right| & \text { peak magnitude of phase- } x \text { to ground instantaneous voltage during post- } \\ & \text { fault time frame at } S S W_{i}, S S W_{i+1}, S S W_{i+2} \text { respectively. }\end{array}$

The zone current change between normal state and fault state can be computed as an index $I_{Z C_{x}^{i}}$ by equation (7.4).

$$
I_{Z C_{x}^{i}}=\frac{I_{Z_{x}^{i}(F)}-I_{Z_{x}^{i}(S)}}{I_{Z_{x}^{i}(S)}} * 100
$$

Where,

$I_{Z C_{x}^{i}}$

$I_{Z_{x}^{i}(F)}$

$I_{Z_{x}^{i}(S)}$

zone current change index of zone- $i$

rms magnitude of zone- $i$ current during post-fault

rms magnitude of zone- $i$ current during pre-fault (steady state)

This stage sets the Zone Current flag $\left(Z C_{f}\right)$ of phase- $x$ status to TRUE, when the zone current change $I_{Z C_{x}^{i}}$ calculated by equation (7.4) is above the pre-defined level $L_{I x}$ for a specified amount of time $T_{I x}$. This detection is done on a per phase basis and " $x$ " denotes the respective phase " $a$ ", " $b$ ", and " $c$ ". The chart in Figure 7-3 shows the Zone Current detection logic. Although the values of $L_{I x}$ and $T_{I x}$ are configurable, the algorithm uses typical values of $200 \%$ (or $2 \mathrm{pu}$ ) and 2 cycles respectively. 


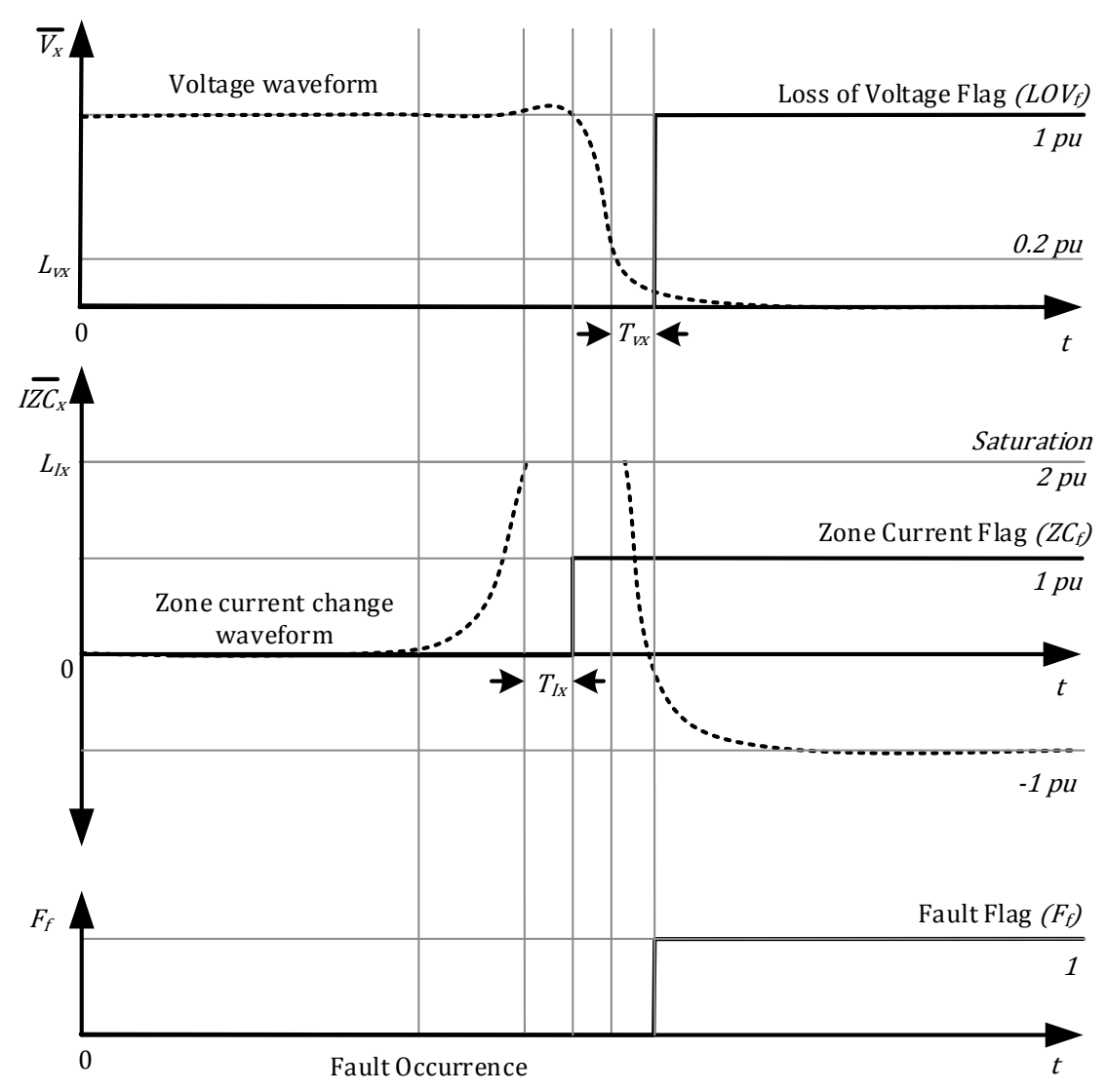

Figure 7-3: Fault Detection Algorithm - Voltage and Zone Current Change Waveforms

\subsubsection{Over Current Fault Detection}

This block determines occurrence of over current fault based on the voltage and zone current information. The Fault Flag $\left(F_{f}\right)$ is set to TRUE when both the $L O V_{f}$ and $Z C_{f}$ flags are TRUE. A counter namely Fault Counter $(F C)$ keeps track of number of times $F_{f}$ is set to TRUE since the last reset. Each time the $F_{f}$ is set to TRUE the $F C$ is incremented by one. This is helpful in determining occurrence of the permanent fault. The logic of the Over Current Fault Detection can be realized by an AND gate implementation as shown in Figure 7-4. 


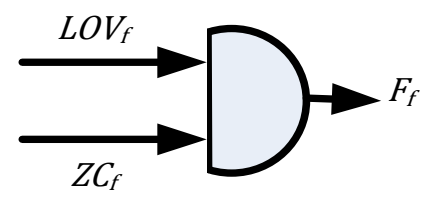

Figure 7-4: AND Gate Implementation of Over Current Fault Detection

\subsubsection{Permanent Fault Determination}

This block determines if there is a permanent over current fault based on the Fault Counter $(F C)$ value. The block sets the Permanent Fault Flag $\left(P F_{f}\right)$ when the $F C$ value is equal to a specified number, which is based on the feeder over current protection employed. The block follows the rules shown below in setting the $P F_{f}$.

$$
P F_{f}=\text { if }\left\{\begin{aligned}
C B \text { protection and } F C & =1 ; & & T R U E \\
\text { Recloser protection and } F C & =(n+1) ; & & \text { TRUE }
\end{aligned}\right.
$$

Where,

$n \quad$ number of recloser switching trials; e.g. for a feeder protected by a recloser with three reclosing trials, the $P F_{f}=$ TRUE when $F C=4$

\subsection{Test System and Modeling}

The distribution system associated with WVSC project is used to test the proposed MAS for Fault Location and Isolation. In order to accurately assess the impact of the short circuit contribution of different types of DGs and MAS fault location algorithm development and testing, a PSCAD model of the WVSC distribution grid is developed. A large-scale inverter-based photovoltaic plant, a synchronous generator, and an induction generator models are developed using the standard components from the PSCAD library. 


\subsubsection{West Virginia Super Circuit (Base Case)}

Figure 7-5 shows a $12.5 \mathrm{kV}$ distribution network of WVSC project fed from two substations WestRun and Pierpont. The substation transformers ratings are 138/12.5 kV and 33.6 MVA each. The feeders WR\#3 and WR\#4 have Distribution Automation and there are 16 automated switches and 2 reclosers installed in these two feeders. Two feeders from Pierpont Substation and three other feeders from WestRun substation are used to restore power to the isolated zones of WR\#3 and WR\#4 feeders.

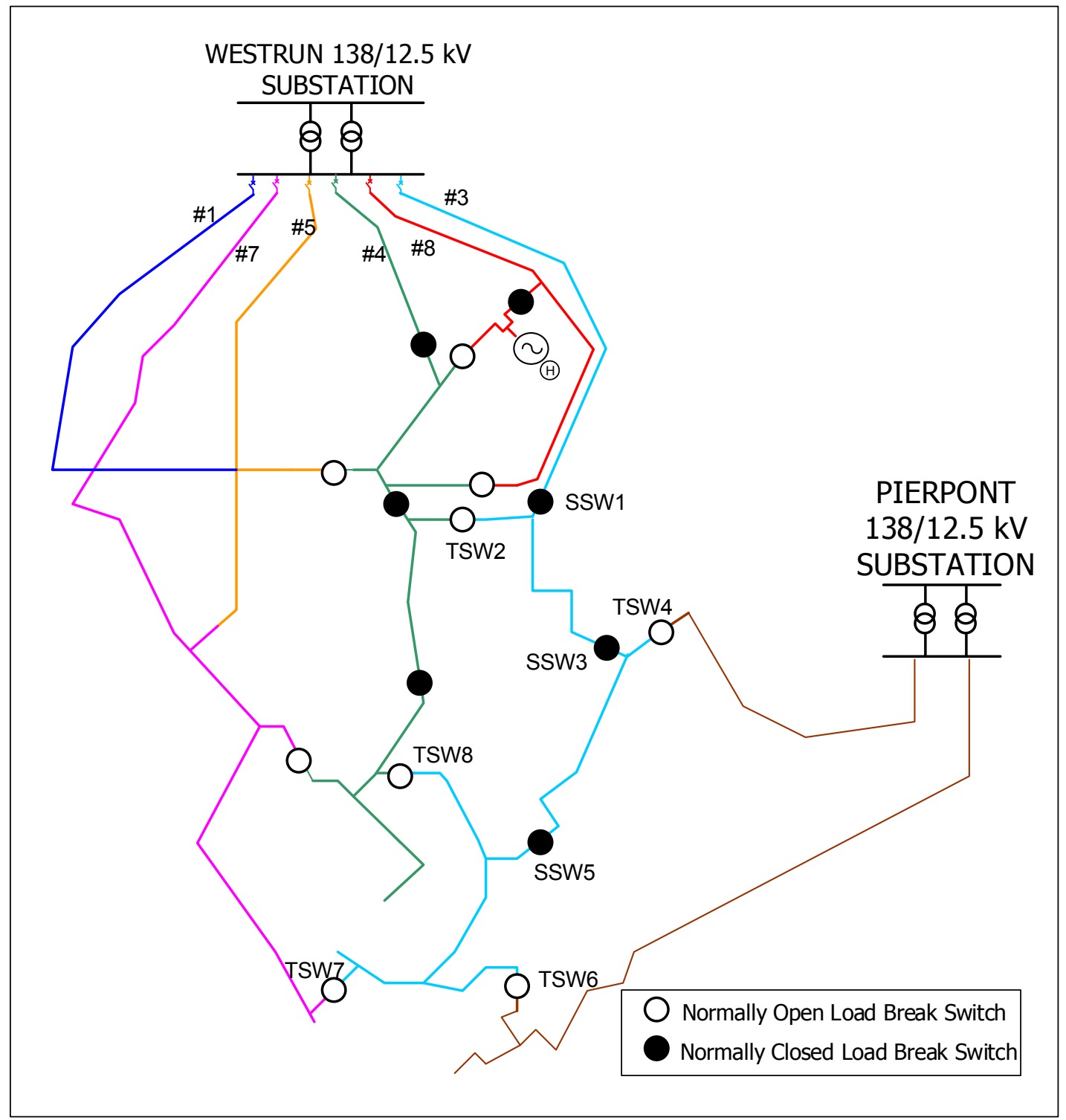

Figure 7-5: Single Line Diagram of WVSC Project 12.5 kV Circuits 


\subsubsection{Automated Switches}

Figure 7-6 shows a pole-top load break switch installed in the WVSC system. The automated switches are Cooper DAS-15 type three-phase vacuum switches with $15 \mathrm{kV}, 630 \mathrm{~A}$ rating. Out of 16 automated switches installed, 7 switches are normally closed and the other 9 switches are used for reconfiguration and restoration applications. A three-phase current transformer set is embedded in these switches, and a three phase set of potential transformers are installed externally on one side of the switch either on the source side or the load side.

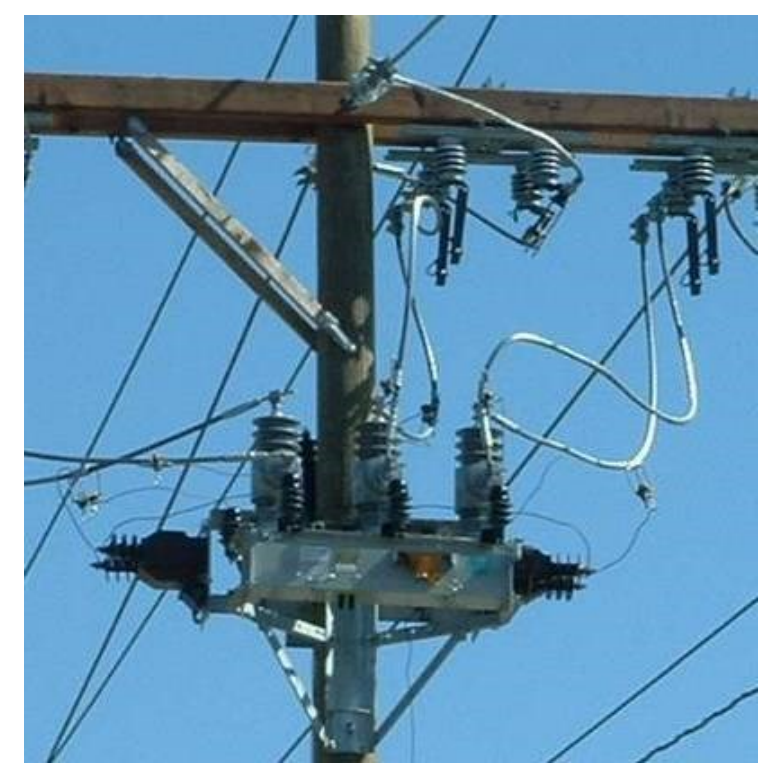

Figure 7-6: Pole-top Load Break Switch

\subsubsection{Substation Reclosers}

All circuits originating from the WestRun substation are equipped with reclosers. WR\#3 and WR\#4 feeders have 560A, $15.5 \mathrm{kV}$ Cooper electronic reclosers with Form 6 controllers on them. The recloser with Cooper Form-6 controller shown in Figure 7-7 takes three shots before permanently locking out on to a permanent fault. Time Current Characteristic (TCC) information for the installed recloser is given in Table 7-1. The first reclosing operates on A-curve (fast curve) and the following two reclosing operations operate on D-curve (slow curve). The approximate 
clearing times for A-curve and D-curve are also listed. The reclosers wait for 60 cycles between each reclosing operation.

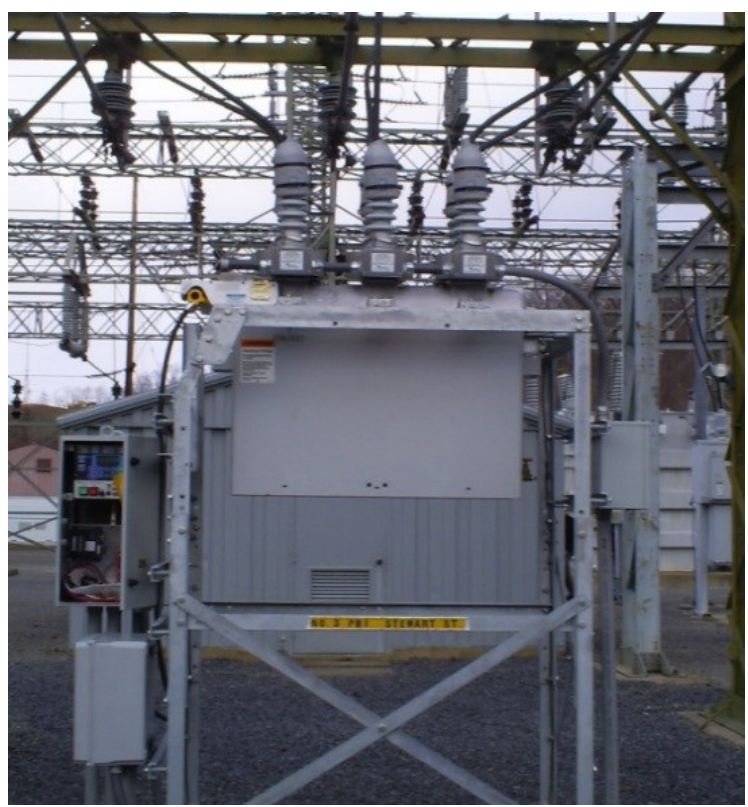

Figure 7-7: Recloser with Cooper Form-6 Controller at WestRun Substation

Table 7-1: Recloser Settings

\begin{tabular}{|c|c|c|c|c|}
\hline & \multicolumn{2}{|c|}{ A curve } & \multicolumn{2}{c|}{ D Curve } \\
\hline Current (A) & 2000 & 6000 & 2000 & 6000 \\
\hline Clearing Time & 5 cycles & 3 cycles & 43 cycles & 8 cycles \\
\hline
\end{tabular}

\subsubsection{Communication Architecture}

The WestRun and Pierpont substations have a Microsol RTU. The West Run RTU communicates with the Pierpont RTU, the Feeder USP RTUs and the Systems Master Station HMI uses DNP TCP/IP protocols for communication. The Substation RTU communicates with the feeder SEL351A relays. The SEL351A relays provide the feeder metering information for all feeders other than WR\#3 and WR\#4, which communicate with Cooper Form6 reclosers for their metering and status data. Hardwired input/output modules on the WestRun RTU provide breaker control for WR\#3 and WR\#4 and substation local/remote indication. The RTU at Pierpont substation only communicates with the WestRun substation RTU, providing information on PP\#5 and PP\#6 
feeders. The WestRun RTU has a DNP TCP/IP master to all 14 feeder RTUs and the Pierpont RTU. A DNP TCP/IP slave communicates with the HMI Master Station.

Each sectionalizing and tie switch point has a Microsol USP RTU communicating with a Microsol AIM-020 meter, which functions as a meter and a fault (over-current) detection device. The USP provides control and indication along with communications to the WestRun Microsol substation RTU. The substation RTU connects to an existing Ethernet 100BaseT (RJ45) access point for Feeder RTU and Master Station communications using DNP TCP/IP. Two RS485 ports are used to connect to the SEL351A relays and in the case of WR\#3 and WR\#4 feeders, the Cooper Form 6s use DNP Serial. The 14 Feeder RTUs connect to a serial to IP converter then to the systems Ethernet communications system using serial DNP. The Feeder RTU communicates to the AIM meter using serial Modbus.

\subsubsection{West Virginia Super Circuit (FLI Test Case)}

In this research, WR\#3 feeder is considered as a test system to prove the proposed MAS FLIR concept. WR\#3 feeder has 3 normally closed load break SSWs creating 4 zones and 5 normally open TSWs. For FLI simulation purpose, four DGs including two synchronous generators, one PV inverter, and one induction generator are introduced into WR\#3 circuit as shown in Figure 7-8. The generators are simulated to produce different levels of output power to change the DER penetration level on the WR\#3 circuit and to provide different testing scenarios for the proposed MAS FLI algorithm. All DGs have associated PCC breakers that are completely complaint with IEEE-1547, i.e., when an islanding condition on the grid occurs, the DG protection opens the PCC breakers within two seconds of the event to prevent unintentional islanding [79]. 


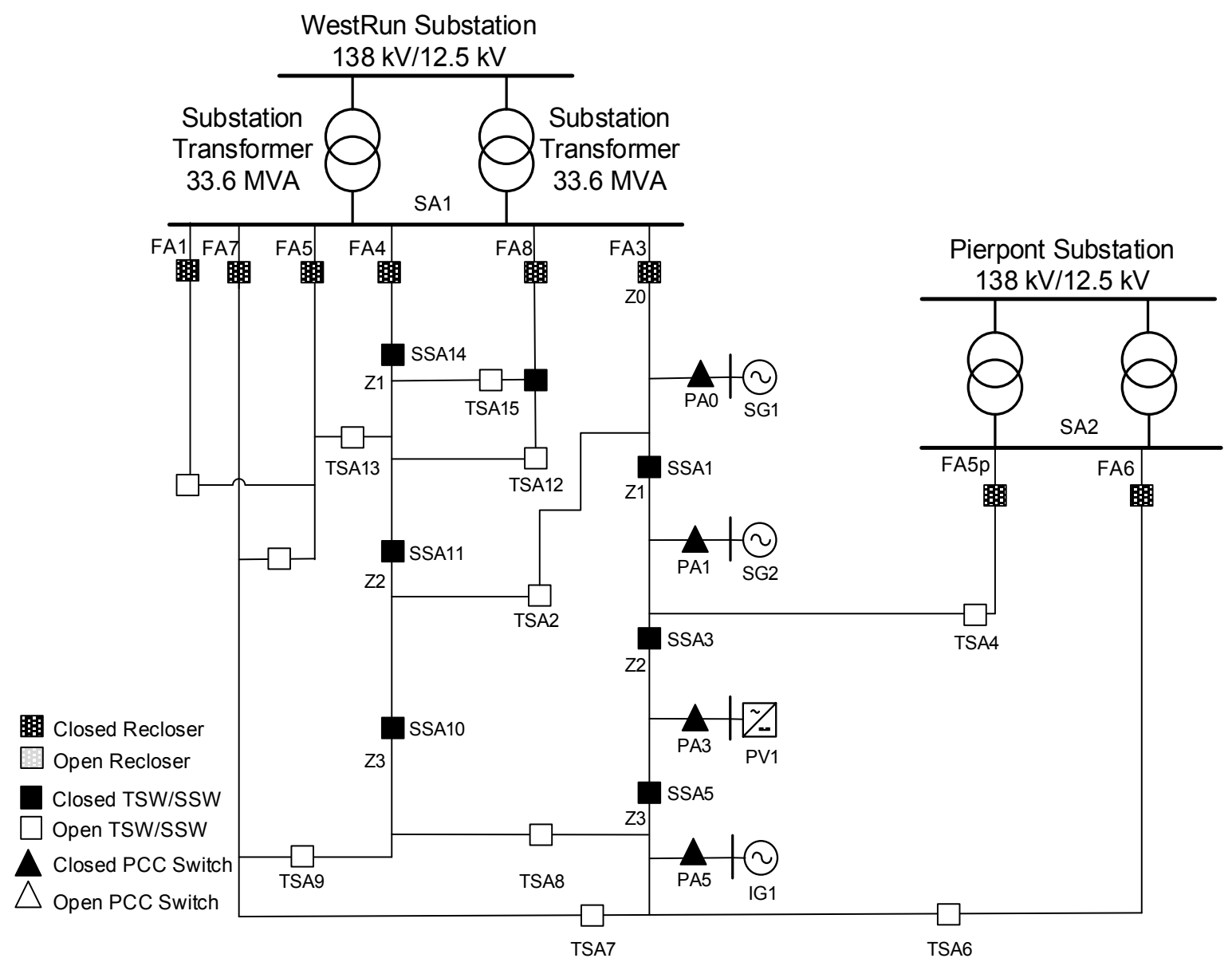

Figure 7-8: Simplified One Line Diagram of WVSC Feeders for MAS FLI

\subsubsection{PSCAD Detailed Modeling}

Figure 7-9 shows the PSCAD model for WR\#3 feeder. Simulation results are calculated based on per unit values. Distribution lines are modeled based on the positive, negative, and zero sequence impedance values. Different types of faults such as single line to ground and three phase faults are modeled with a fault block in PSCAD library. Feeder loads in each zone are modeled as constant active and reactive power loads. Annual peak demand on WR\#3 feeder is modeled as $5 \mathrm{MW}$ at 95\% power factor. The simulations are carried out in a discrete mode with a discrete step simulation time of 16.67 micro-seconds in order to analyze the transient DG fault characteristics. This results in 1000 samples per cycle simulation readings. 


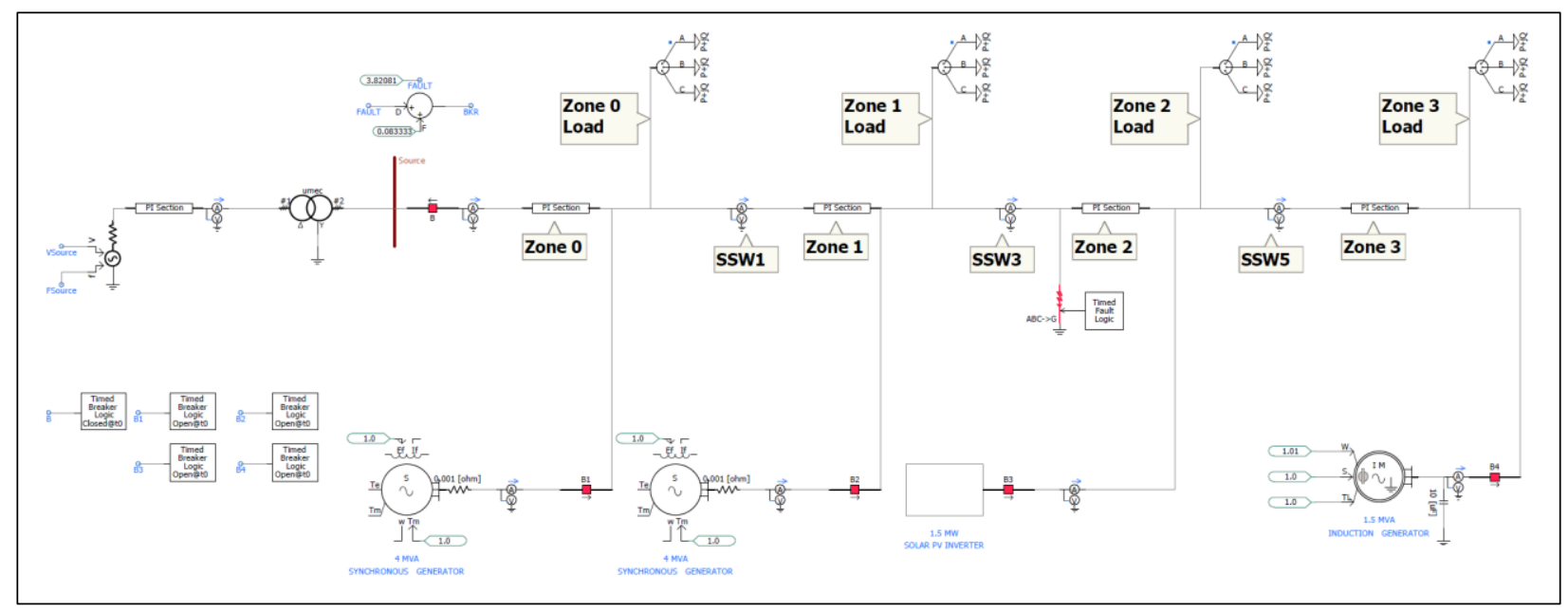

Figure 7-9: WVSC Distribution Feeder WR\#3 PSCAD Model

\subsubsection{PV Inverter Model}

Figure 7-10 shows the PSCAD core model of the photovoltaic inverter used for this study. It is a full scale representation of a voltage source inverter with Pulse-Width Modulation (PWM). The inverter bridge is followed by a LC filter to take out harmonic distortion and allow a controllable power flow. PWM inverters can quickly cease delivering power to the grid by stopping gating signals going to the inverter. With switching frequencies of a few kilohertz, this provides tens of opportunities for disconnection in a single cycle. When dealing with short circuits, the two decision-making mechanisms for disconnection are under-voltage and over-current measurements.

An important consideration with inverters is the presence of an instantaneous over current relay (ANSI function \#50). Solid-state devices being acutely sensitive to over current, manufacturers must equip their converters with fast over current sensors to avoid the power bridge to self-destruct at the first abnormal situation.

The breaker "B" facilitates the connection to the electric grid and has the standard voltage and frequency protection settings specified in IEEE 1547 standard as shown in Table 7-2 and Table 7-3. 
Table 7-2: Interconnection System Response to Abnormal Voltages (rms Voltage)

\begin{tabular}{ccc}
\hline $\begin{array}{c}\text { Voltage Range } \\
(\% \text { of the base voltage) }\end{array}$ & $\begin{array}{c}\text { Protective Function } \\
\text { (ANSI \#) }[80]\end{array}$ & $\begin{array}{c}\text { Maximum Clearing } \\
\text { Time (s) }\end{array}$ \\
\hline $\mathrm{V}<50$ & Under voltage (27) & 0.16 \\
$50 \leq \mathrm{V}<88$ & Under voltage (27) & 2.00 \\
$110<\mathrm{V}<120$ & Over voltage (59) & 1.00 \\
$\mathrm{~V} \geq 120$ & Over voltage (59) & 0.16 \\
\hline
\end{tabular}

Table 7-3: Interconnection System Response to Abnormal Frequencies

\begin{tabular}{ccc}
\hline Frequency Range (Hz) & $\begin{array}{c}\text { Protective Function } \\
\text { (ANSI \#) }[80]\end{array}$ & $\begin{array}{c}\text { Maximum Clearing } \\
\text { Time (s) }\end{array}$ \\
\hline $\mathrm{f}>60.5$ & Over Frequency (81) & 0.16 \\
$\mathrm{f}<57.0$ & Under Frequency (81) & 0.16 \\
\hline
\end{tabular}

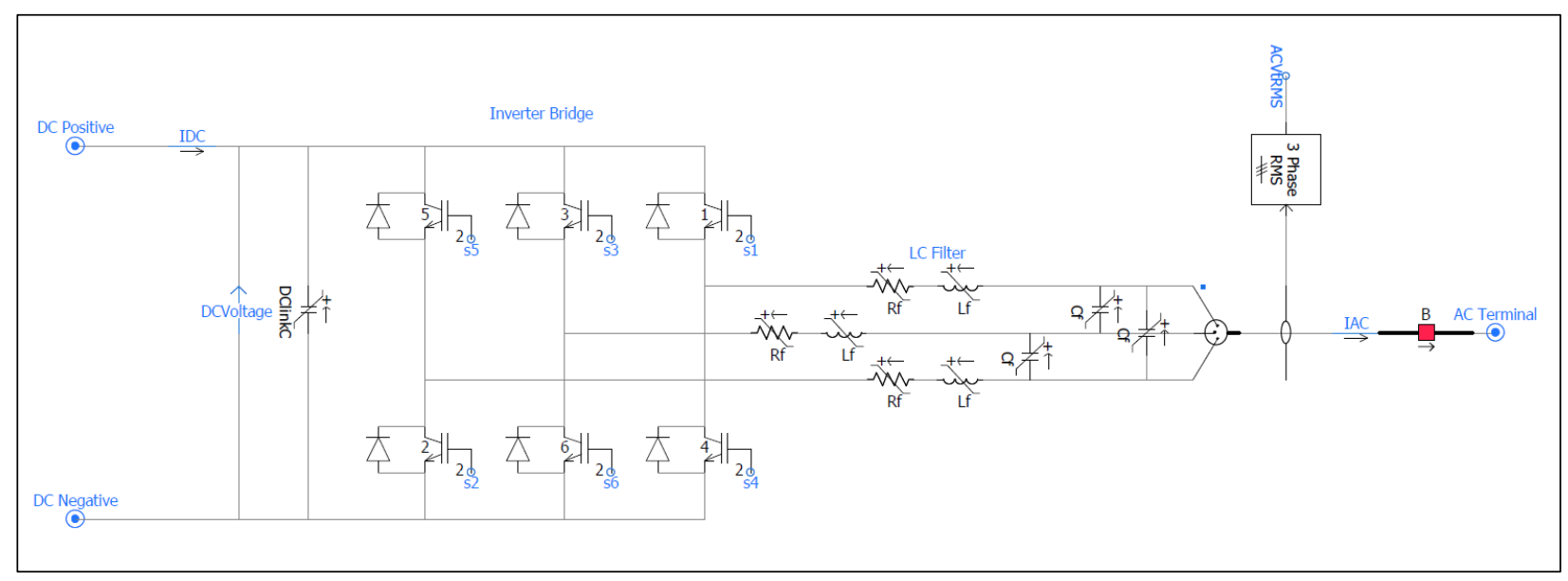

Figure 7-10: PSCAD Model of PV Inverter

\subsubsection{Synchronous Generator Model}

A simulation model of a synchronous machine based DG unit is developed for the study. The model represents a small 4 MVA hydro DG based on a synchronous generator. The resulting PSCAD model is shown in Figure 7-11. The model uses standard components from the PSCAD library. Multi-mass representation and/or shaft torsional dynamics are neglected in this model 
since the generator is small and sub-synchronous resonances are not of interest for this study. A tie-breaker is installed at the connection point of DG and used to disconnect the unit.

The synchronous generator is initialized with the grid steady state conditions such as POI voltage magnitude and phase angle. The governor and exciter models are eliminated and assumed a constant field voltage of $1.0 \mathrm{pu}$ to produce terminal voltage of $1.0 \mathrm{pu}$. The synchronous generator is made to run at constant speed by locking the rotor at synchronous speed. Thus, there are no prime mover dynamics involved. To make the model simpler, the machine saturation is disabled in the model. These simplifications allowed us to focus primarily on the machine dynamics during the fault event.

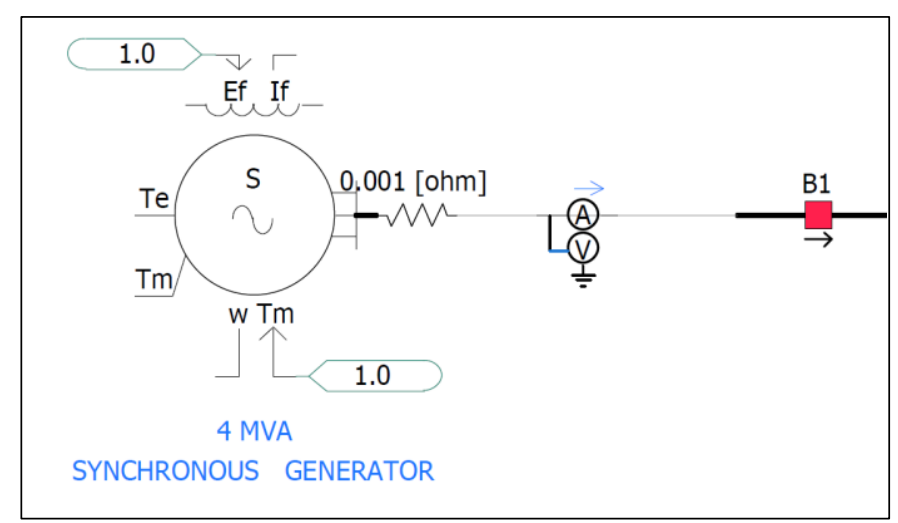

Figure 7-11: Synchronous Generator PSCAD Model

\subsubsection{Induction Generator Model}

A simulation model of an induction machine based DG unit is developed for the study. The model represents a small 1.5 MVA induction generator. The resulting PSCAD model is shown in Figure 7-12. The model uses standard components from the PSCAD library. The induction generator model can be controlled by specifying either the mechanical torque $\left(\mathrm{T}_{\mathrm{L}}\right)$, or by machine speed $(\mathrm{W})$. The input " $\mathrm{S}$ " is simply a switch signal used to select between two input modes, i.e., when $\mathrm{S}=0$, input signal $T_{L}$ is selected, and if $S=1$ input signal $W$ is selected. In order to operate the machine in speed mode, $S=1$ is used in the model. A shunt capacitor is used to compensate for the reactive power demand of the induction generator. A tie-breaker is installed at the connection point of DG and used to disconnect the unit. 


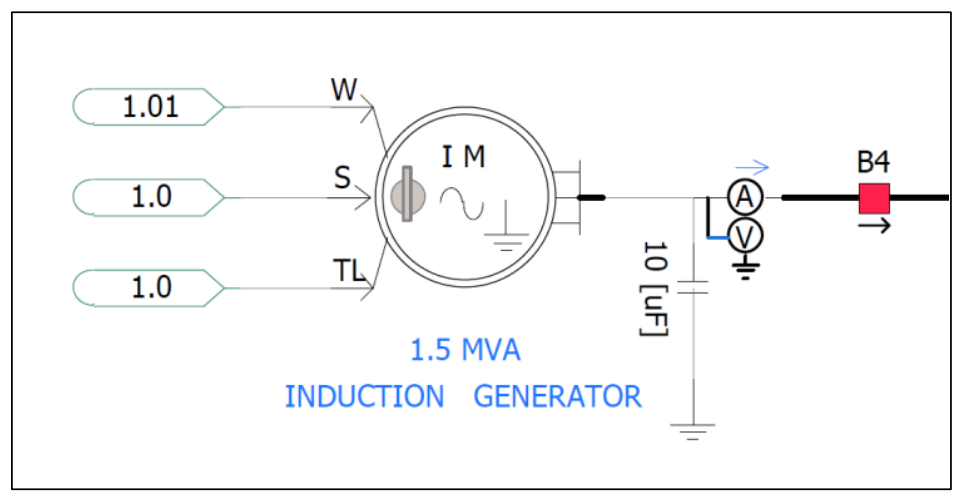

Figure 7-12: Induction Generator PSCAD Model

\subsection{Fault Location \& Isolation Results}

\subsubsection{Case I - Three Phase Fault in Zone-2}

In this scenario a permanent three phase fault (LLL fault) at time 3.82 seconds is considered in Zone-2 (between SSW3 and SSW5) of WR\#3 feeder. The feeder recloser detects the over current and operates based on TCC information provided in Table 7-1 and locks out. The generator breakers for SG1, SG2, and IG1 open up five cycles after the fault occurrence, i.e. at 3.90 seconds. The PV inverter breaker trips at 3.97 seconds following the IEEE 1547 protection settings given in Table 7-2 and Table 7-3 .

The DGs connected in different zones operate in a grid paralleled mode and supplying feeder loads during the pre-fault condition. The pre-fault DG output powers and DG instantaneous fault contributions are shown in Figure 7-13 and Figure 7-14. Table 7-4 summarizes the DG output power $(\mathrm{kW})$ and fault contribution $(\mathrm{pu})$ measurements. This case represents a $80 \% \mathrm{DG}$ penetration scenario as the total DG output power is close to $4.0 \mathrm{MW}$ compared to the total feeder load of 5 MW.

The fault contribution values are per unitized using the individual machine nominal kVA rating as base. The DG fault contribution values align with the standard fault contribution values of their machine type that are described in Chapter 6. The PV inverter fault contribution is almost close to 
$2.3 \mathrm{pu}$ right after the fault occurrence and it settles close to $1.7 \mathrm{pu}$ few cycles after the fault occurrence.

The permanent fault flag $\left(P F_{f}\right)$ of SSA3 for three phases are set to TRUE based on the Fault Detection algorithm. The per-phase zone current change $(\%)$ and voltage $(\mathrm{kV})$ waveforms, fault flag, and permanent fault flag at SSA3 are shown in Figure 7-15. Figure 7-16 shows the per phase instantaneous voltages at the substation and the SSW nodes. It clearly shows that the magnitude of voltage during the post-fault condition at the SSW3 location is the lowest in the feeder.

Table 7-5 tabulates the Fault Counter $(F C)$ values and Permanent Fault Flag $\left(P F_{f}\right)$ status for SSA1, SSA3, and SSA5.

The agent communications that takes place for the fault location and isolation is listed below and shown in Figure 7-17. The SSA3 concludes that there is a three phase fault occurred between its node and down-stream node and isolates the fault by opening SSW3 and SSW5.

1. Recloser is locked out on a permanent fault (LLL fault).

2. FA3 sends REQUEST performative messages to SSA1, SSA3, and SSA5 to start fault location.

3. SSA1, SSA3, and SSA5 checks their $P F_{f}$ flag status.

4. SSA3 finds out the $P F_{f}$ status for all three phases are set to TRUE and it determines that there is a three phase fault between its node and downstream node, i.e., Zone-2 (between SSW3 and SSW5).

5. SSA3 sends a INFORM performative message to FA3 to indicate the fault location.

6. SSA3 puts SSW3 in OPEN-LOCK status.

7. SSA3 sends a REQUEST performative messages to SSA5 to put SSW5 in OPEN-LOCK state.

8. SSA5 sends a $A C C E P T$ performative messages to SSA3 after SSW5 is in OPEN-LOCK state.

9. SSA3 sends a INFORM performative messages to FA3 to indicate completion of fault isolation. 
Table 7-4: DG Output Power and Fault Contribution

\begin{tabular}{cccccc}
\hline \# & DG Name & Zone & $\begin{array}{c}\text { DG Rating } \\
(\mathbf{k V A})\end{array}$ & $\begin{array}{c}\text { DG Output Power } \\
(\mathbf{k W})^{\mathbf{1}}\end{array}$ & Fault Contribution (pu) $^{\mathbf{2}}$ \\
\hline 1 & SG1 & Z0 & 4000 & 850 & 5.1 \\
2 & SG2 & Z1 & 4000 & 850 & 5.1 \\
3 & PV & Z2 & 1500 & 1000 & 2.4 \\
4 & IG1 & Z3 & 1500 & 1300 & 6.4 \\
\hline
\end{tabular}

1. DG output power measured at pre-fault condition.

2. Peak instantaneous fault contribution per unitized using the machine base.

Table 7-5: Fault Counter Value and Permanent Fault Flag Status for LLL Fault in Zone-2

\begin{tabular}{c|ccc|ccc|ccc}
\hline & \multicolumn{3}{|c|}{ SSA1 } & \multicolumn{3}{c|}{ SSA3 } & & SSA5 \\
\hline & Ph-A & Ph-B & Ph-C & Ph-A & Ph-B & Ph-C & Ph-A & Ph-B & Ph-C \\
\hline $\begin{array}{c}\text { Fault Counter } \\
(\boldsymbol{F C})\end{array}$ & 0 & 0 & 0 & 4 & 4 & 4 & 0 & 0 & 0 \\
$\begin{array}{c}\text { Permanent Fault } \\
\text { Flag }\left(\boldsymbol{P F}_{\boldsymbol{f}}\right)\end{array}$ & FALSE & FALSE & FALSE & TRUE & TRUE & TRUE & FALSE & FALSE & FALSE \\
\hline
\end{tabular}




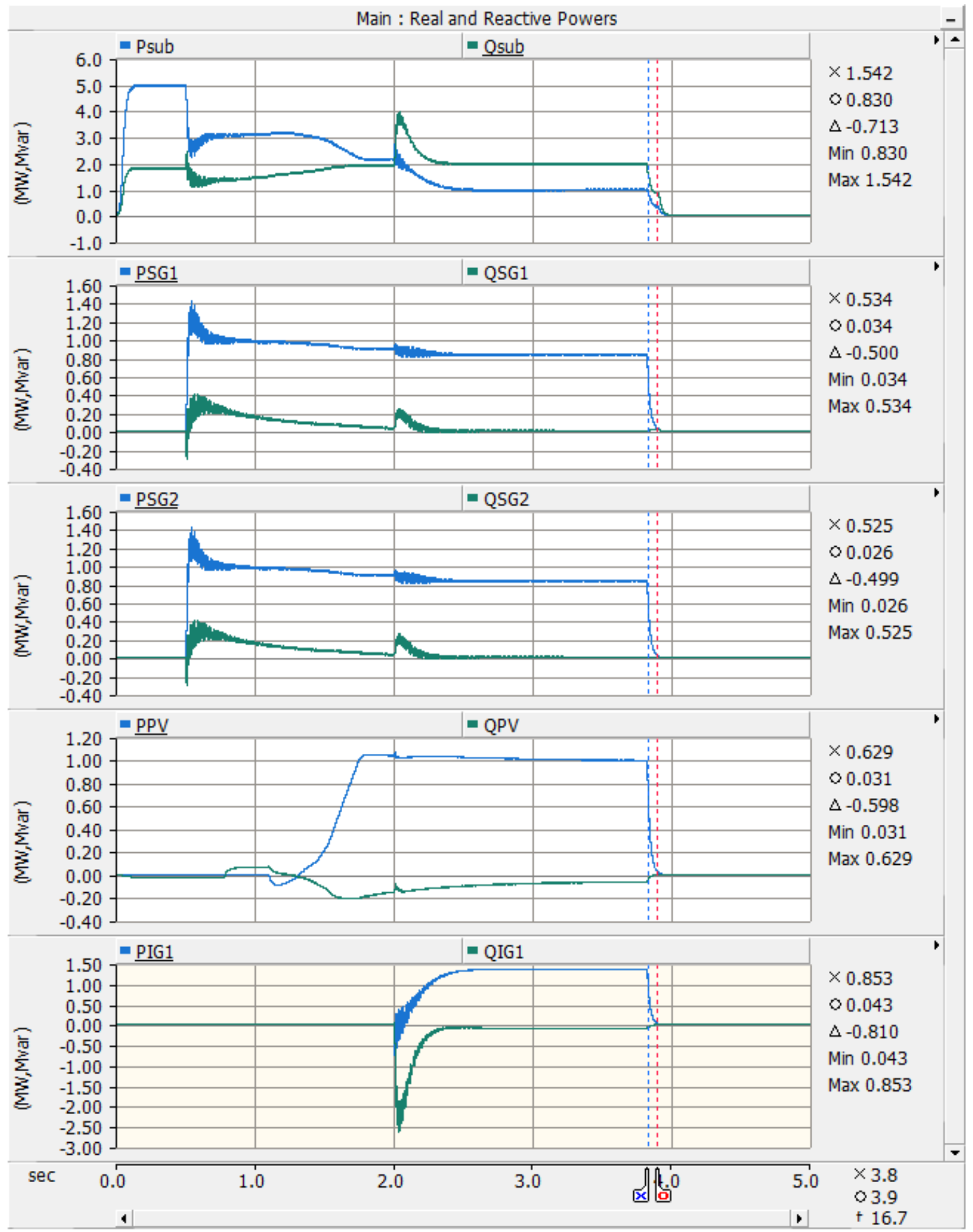

Figure 7-13: DG Real and Reactive Power Outputs 


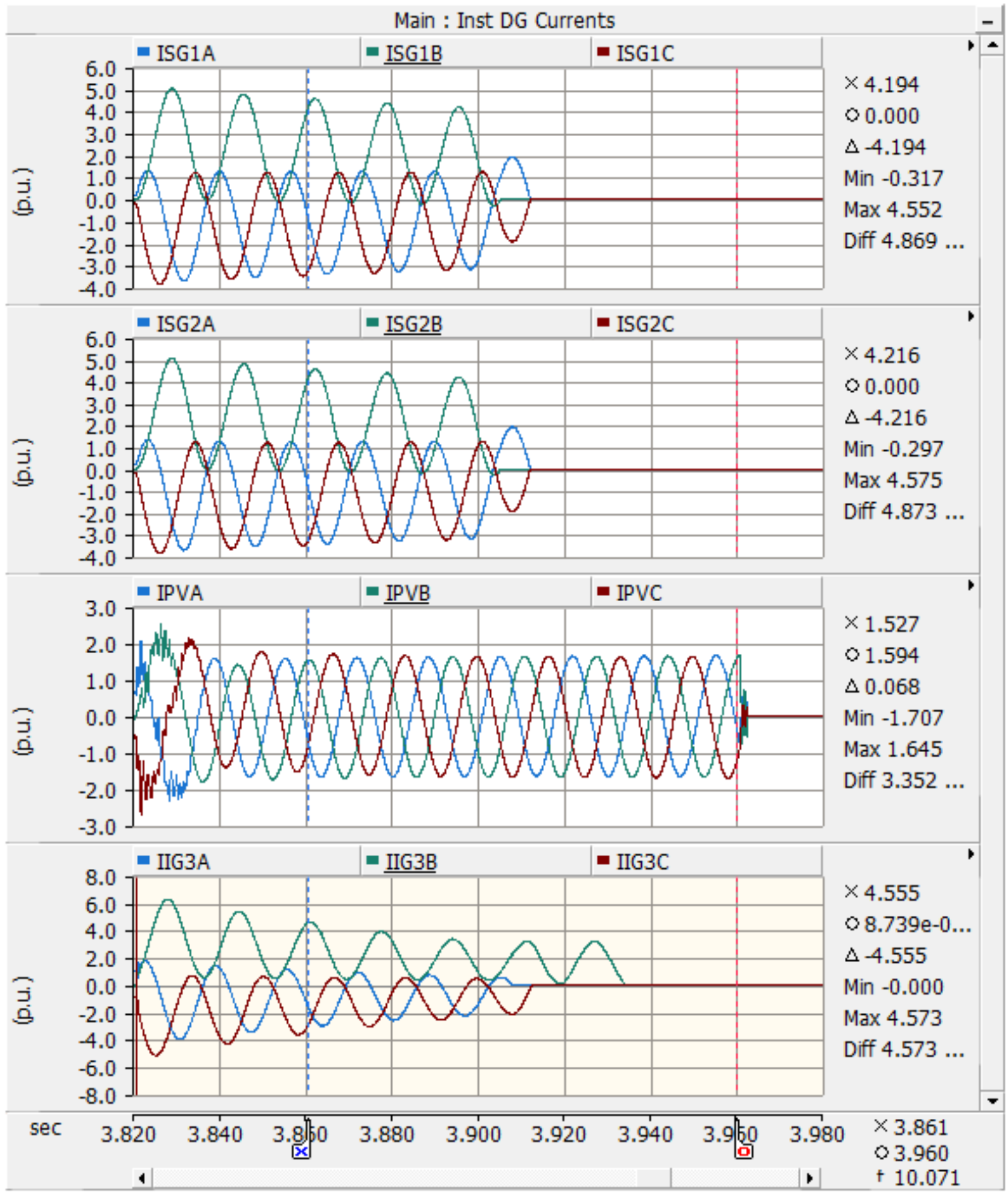

Figure 7-14: DG Instantaneous Fault Contributions 


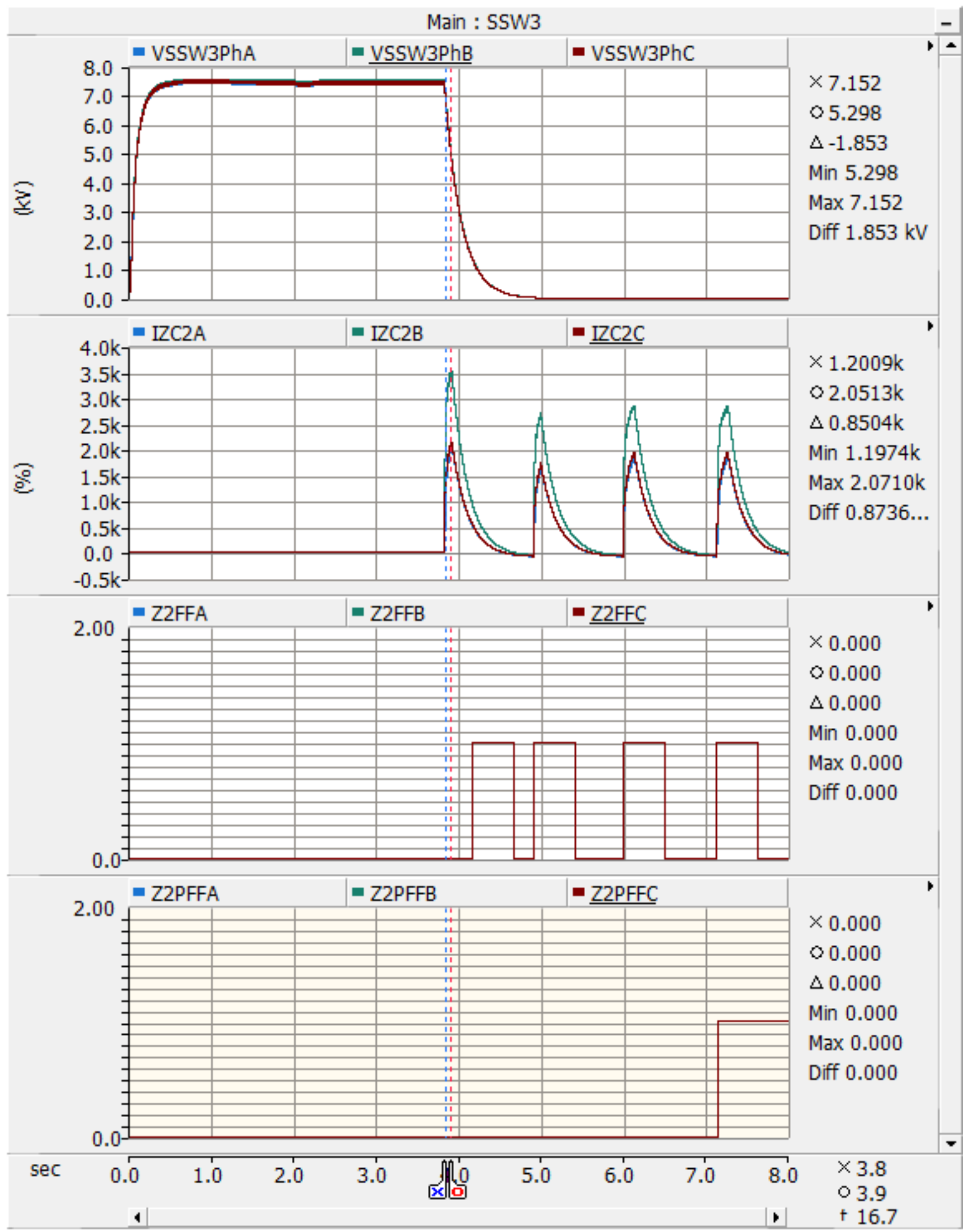

Figure 7-15: SSW3 Measurements (Voltages, Zone Current Change, Fault Flag, and Permanent Fault Flag) 


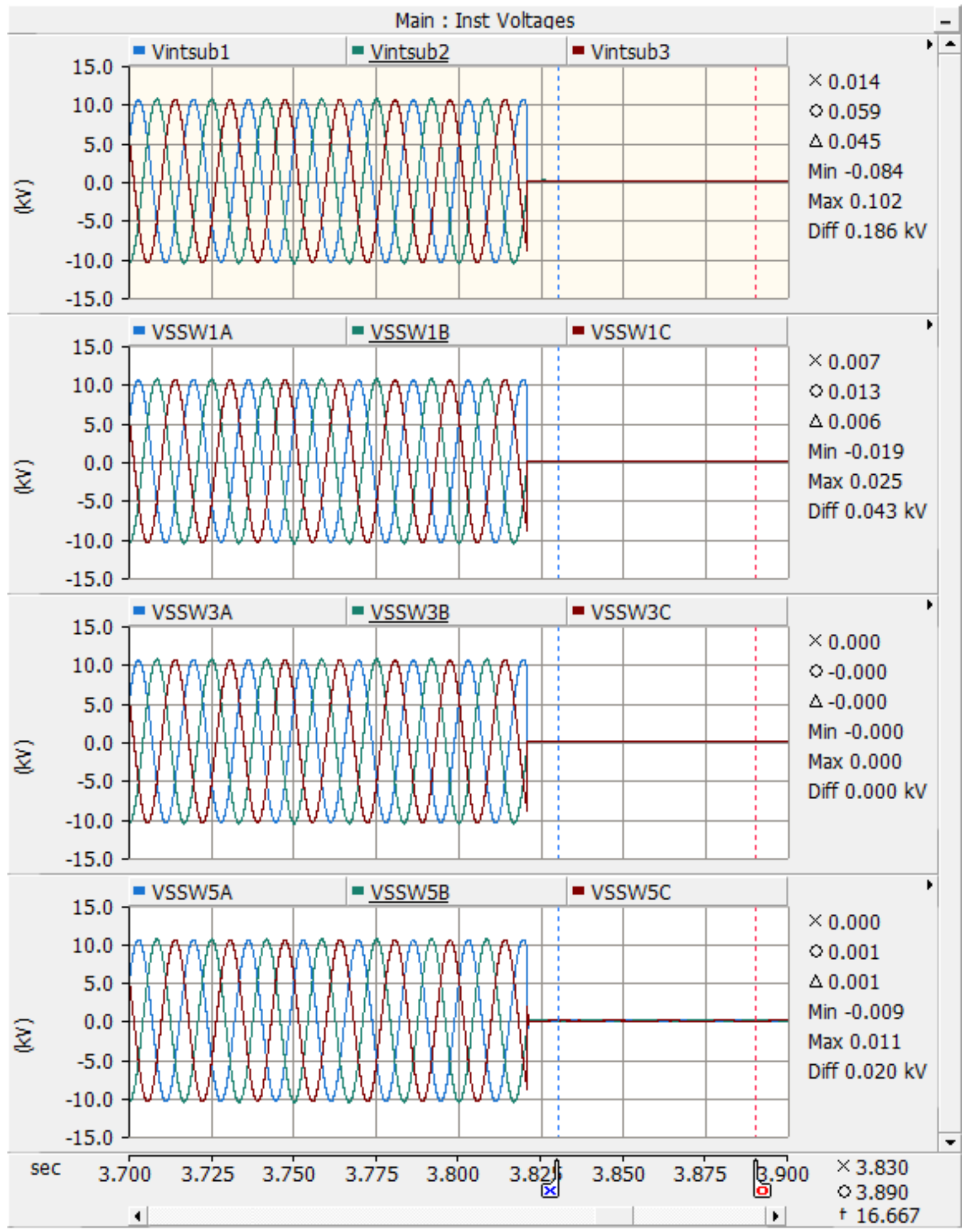

Figure 7-16: Instantaneous Voltages at the Substation and SSW locations 


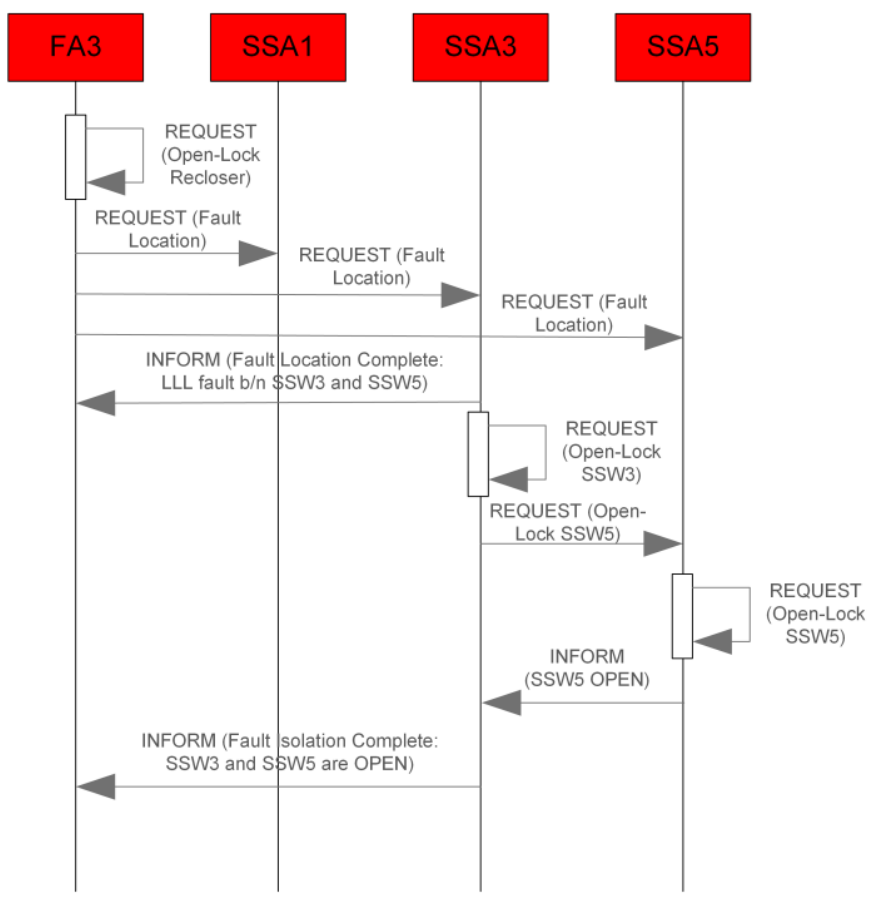

Figure 7-17: Agent Communications for Fault Location and Isolation for LLL Fault in Zone-2

\subsubsection{Case II - Line to Ground (LG-A) Fault in Zone-1}

In this scenario a permanent phase-A to ground fault (LG-A fault) at time 3.82 seconds is considered in Zone-1 (between SSW1 and SSW3) of WR\#3 feeder. The feeder recloser detects the over current and operates based on the TCC information provided in Table 7-1 and locks out. The generator breakers for SG1, SG2, and IG1 open up five cycles after the fault occurrence, i.e. at 3.90 seconds. The PV inverter breaker trips at 4.10 seconds following the IEEE 1547 protection settings given in Table 7-2 and Table 7-3.

The DGs connected in different zones operate in a grid paralleled mode and supplying feeder loads during the pre-fault condition. The pre-fault DG output powers and DG instantaneous fault contribution are shown in Figure 7-18 and Figure 7-19. Table 7-6 summarizes the DG output power $(\mathrm{kW})$ and fault contribution $(\mathrm{pu})$ measurements. This case represents a 90\% DG penetration scenario as the total DG output power is close to $4.5 \mathrm{MW}$ compared to the total feeder load of 5 MW. 
The fault contribution values are per unitized using the individual machine nominal $\mathrm{kVA}$ rating as base. The DG fault contribution values align with the standard fault contribution values of their machine type that are described in Chapter 6. The PV inverter fault contribution is close to $2.3 \mathrm{pu}$ right after the fault occurrence and it settles close to $1.7 \mathrm{pu}$ few cycles after the fault occurrence.

The permanent fault flag $\left(P F_{f}\right)$ of SSA1 for phase-A is set to TRUE based on the Fault Detection algorithm. The per-phase zone current change $(\%)$ and voltage $(\mathrm{kV})$ waveforms, fault flag, and permanent fault flag at SSA1 are shown in Figure 7-20. Figure 7-21 shows the per phase instantaneous voltages at the substation and at the SSW nodes. It clearly shows that the magnitude of voltage during the post-fault condition at the SSW1 location is the lowest in the feeder. Table 7-7 tabulates the Fault Counter $(F C)$ values and Permanent Fault Flag $\left(P F_{f}\right)$ status for SSA1, SSA3, and SSA5.

The agent communications that take place for the fault location and isolation is listed below and shown in Figure 7-22. The SSA1 concludes that there is a LG-A fault between its node and downstream node and isolates the fault by opening SSW1 and SSW3.

1. Recloser is locked out on a permanent fault (LG-A fault).

2. FA3 sends REQUEST performative messages to SSA1, SSA3, and SSA5 to start fault location.

3. SSA1, SSA3, and SSA5 checks their $P F_{f}$ flag status.

4. SSA1 finds out the $P F_{f}$ status for phase-A is set to TRUE and it determines that there's a LG-A fault between its node and downstream node, i.e., Zone-1 (between SSW1 and SSW3).

5. SSA1 sends a INFORM performative message to FA3 to indicate the fault location.

6. SSA1 puts SSW1 in OPEN-LOCK status.

7. SSA 1 sends a REQUEST performative messages to SSA 3 to put SSW3 in OPEN-LOCK state.

8. SSA3 sends a $A C C E P T$ performative messages to SSA1 after SSW3 is in OPEN-LOCK state.

9. SSA1 sends a INFORM performative messages to FA3 to indicate completion of fault isolation. 
Table 7-6: DG Output Power and Fault Contribution

\begin{tabular}{cccccc}
\hline \# & DG Name & Zone & $\begin{array}{c}\text { DG Rating } \\
(\mathbf{k V A})\end{array}$ & $\begin{array}{c}\text { DG Output Power } \\
(\mathbf{k W})^{\mathbf{1}}\end{array}$ & Fault Contribution (pu) $^{\mathbf{2}}$ \\
\hline 1 & SG1 & Z0 & 4000 & 825 & 2.7 \\
2 & SG2 & Z1 & 4000 & 825 & 2.7 \\
3 & PV & Z2 & 1500 & 1500 & 2.3 \\
4 & IG1 & Z3 & 1500 & 1350 & 6.5 \\
\hline
\end{tabular}

1. DG output power measured at pre-fault condition.

2. Peak instantaneous fault contribution per unitized using the machine base.

Table 7-7: Fault Counter Value and Permanent Fault Flag Status for LG-A fault in Zone-1

\begin{tabular}{c|ccc|ccc|ccc}
\hline & \multicolumn{3}{|c|}{ SSA1 } & \multicolumn{3}{c|}{ SSA3 } & & SSA5 \\
\hline & Ph-A & Ph-B & Ph-C & Ph-A & Ph-B & Ph-C & Ph-A & Ph-B & Ph-C \\
\hline $\begin{array}{c}\text { Fault Counter } \\
(\boldsymbol{F C}) \\
\begin{array}{c}\text { Permanent Fault } \\
\text { Flag }\left(\boldsymbol{P} \boldsymbol{F}_{\boldsymbol{f}}\right)\end{array}\end{array}$ & 4 & 0 & 0 & 0 & 0 & 0 & 0 & 0 & 0 \\
\hline
\end{tabular}




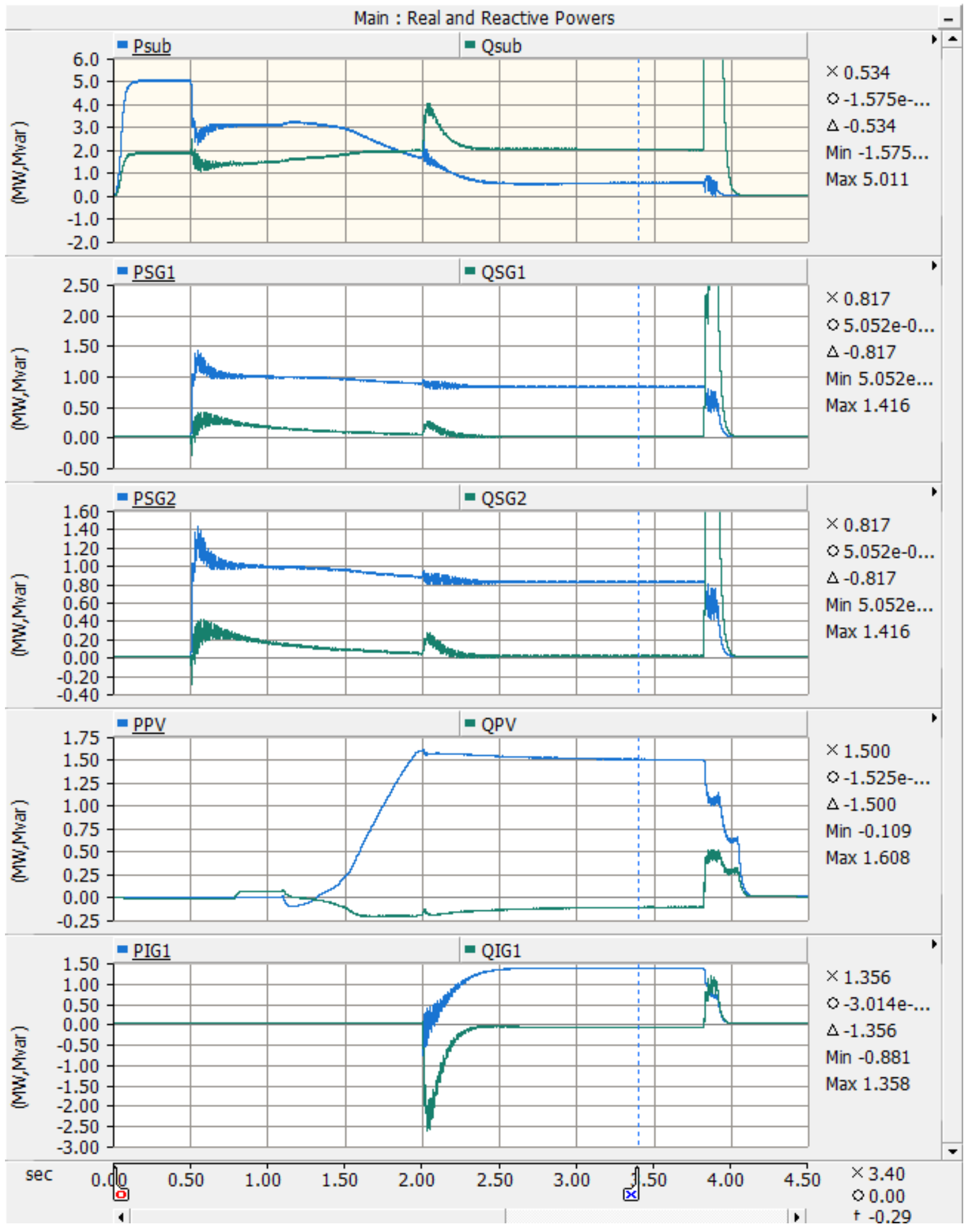

Figure 7-18: DG Real and Reactive Power Outputs 


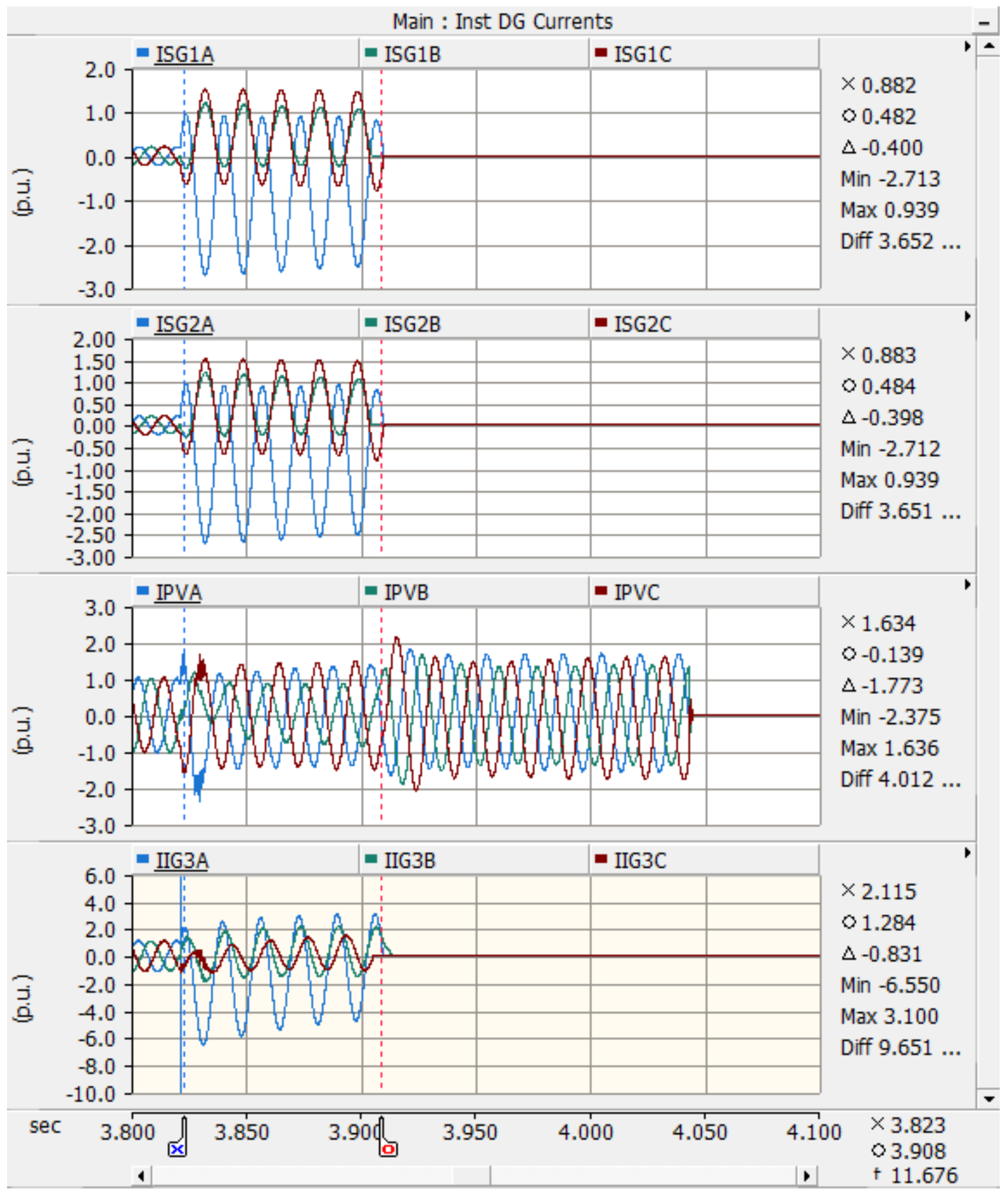

Figure 7-19: DG Instantaneous Fault Contributions 


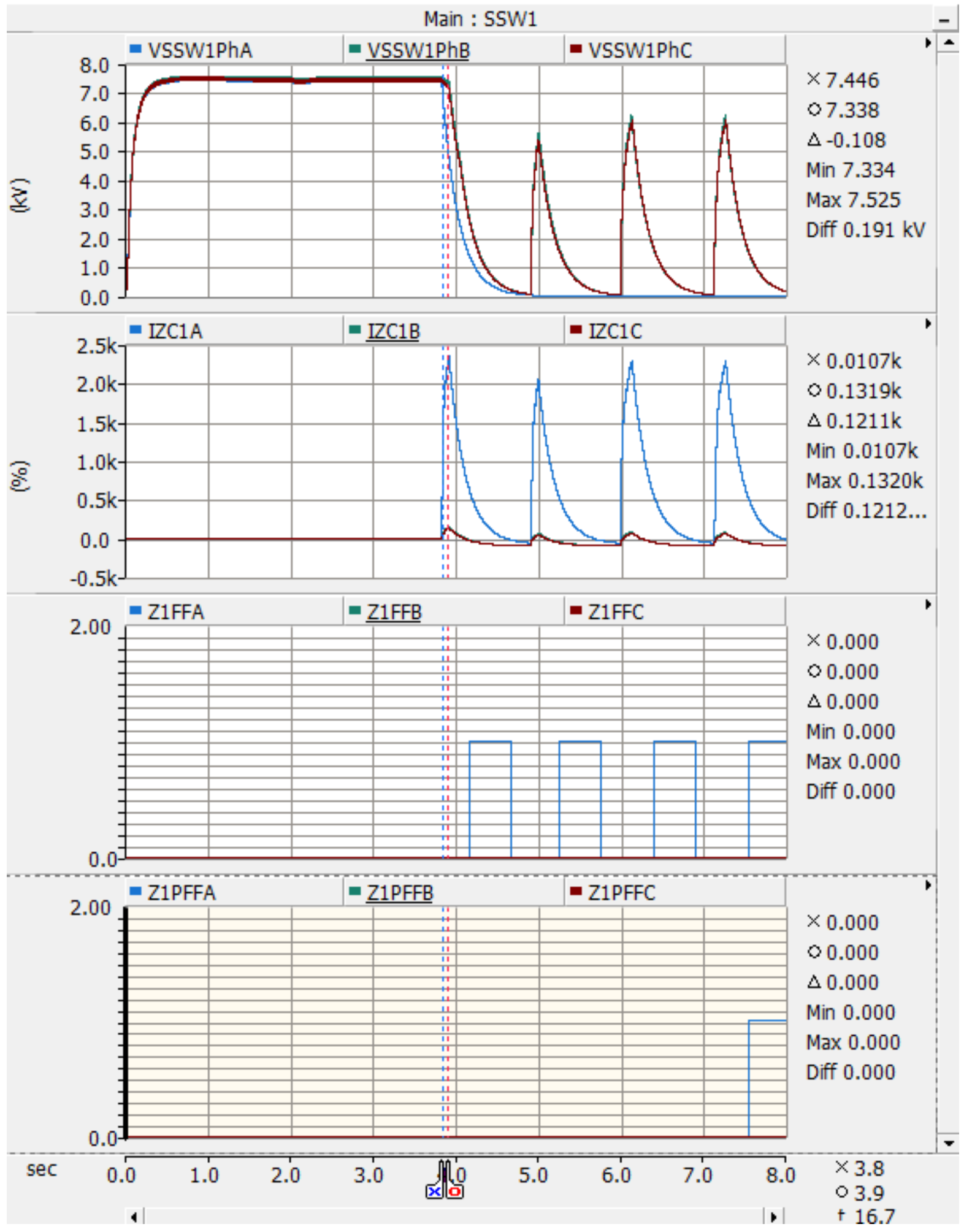

Figure 7-20: SSW1 Measurements (Voltages, Zone Current Change, Fault Flag, and Permanent Fault Flag) 


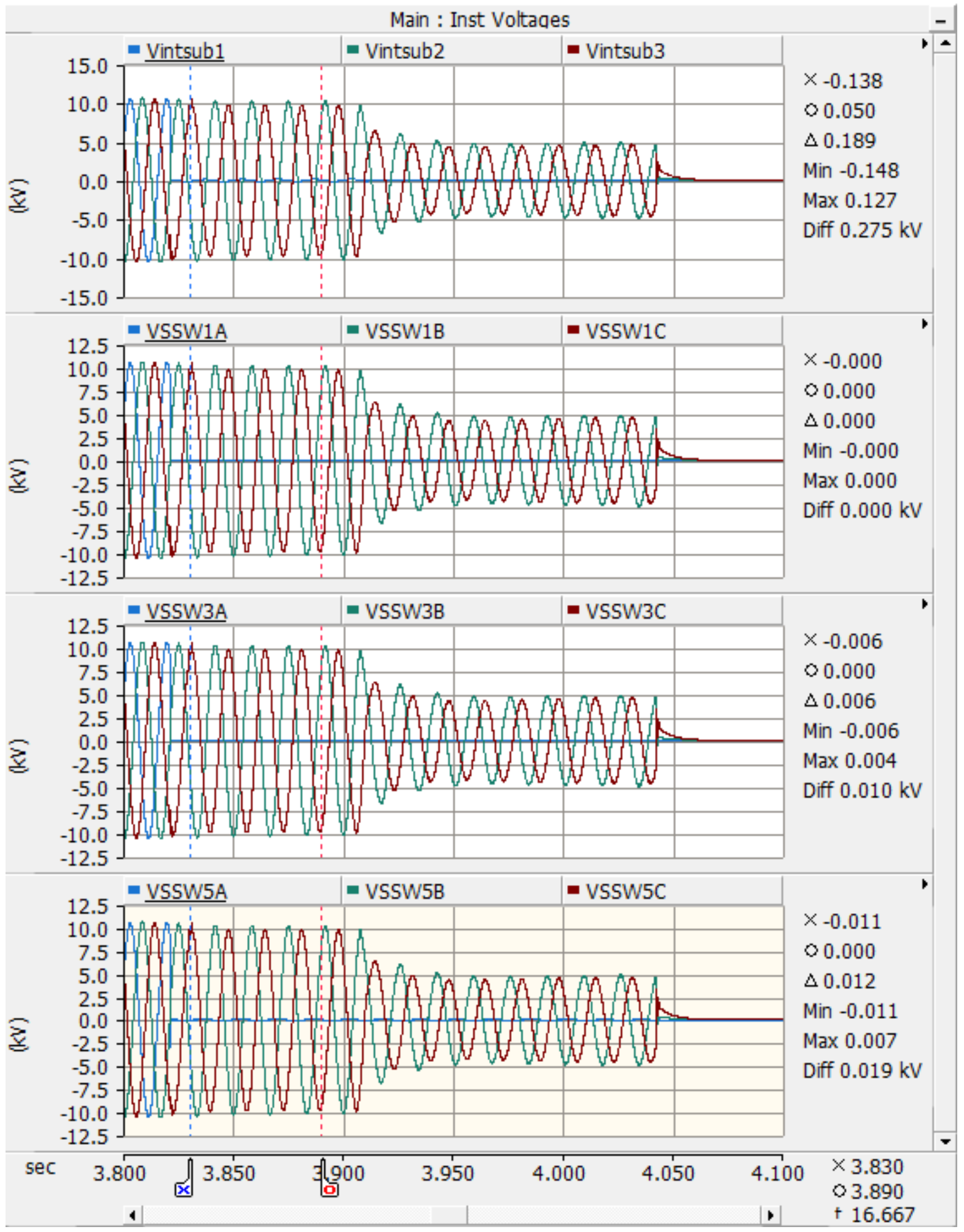

Figure 7-21: Instantaneous Voltages at the Substation and SSW locations 


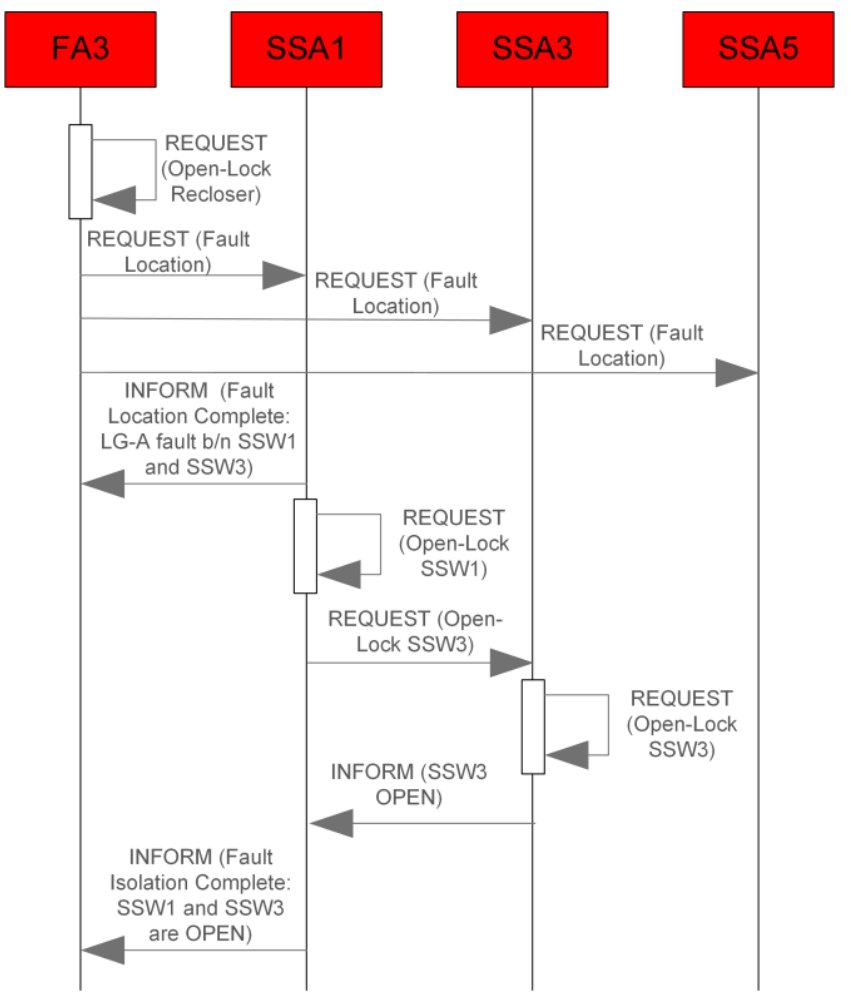

Figure 7-22: Agent Communications for Fault Location and Isolation for LG-A Fault in Zone-1

\subsubsection{Miscellaneous Faults}

The proposed MAS logic for fault location and isolation has been tested for various fault types in multiple zones of WR\#3 feeder with varying DG penetration levels up to $100 \%$ of the feeder load. Table 7-8 below tabulates $F C$ values and $P F_{f}$ status for SSA1, SSA3, and SSA5 for various fault scenarios. This proves that the proposed logic can identify fault type and locate faults in all zones irrespective of DG penetration on a radial distribution feeder. 
Table 7-8: Fault Location Results for Miscellaneous Faults

\begin{tabular}{|c|c|c|c|c|c|c|c|c|c|c|}
\hline \multirow{3}{*}{ \# } & \multirow{3}{*}{ Fault Scenario } & \multicolumn{3}{|c|}{ SSA1 } & \multicolumn{3}{|c|}{ SSA3 } & \multicolumn{3}{|c|}{ SSA5 } \\
\hline & & \multicolumn{9}{|c|}{ Fault Counter $(F C) /$ Permanent Fault Flag $\left(P F_{f}\right)$} \\
\hline & & $\mathrm{Ph}-\mathrm{A}$ & $\mathrm{Ph}-\mathrm{B}$ & $\mathrm{Ph}-\mathrm{C}$ & $\mathrm{Ph}-\mathrm{A}$ & $\mathrm{Ph}-\mathrm{B}$ & $\mathrm{Ph}-\mathrm{C}$ & $\mathrm{Ph}-\mathrm{A}$ & $\mathrm{Ph}-\mathrm{B}$ & $\mathrm{Ph}-\mathrm{C}$ \\
\hline \multicolumn{11}{|c|}{$0 \%$ DG Penetration } \\
\hline 1 & LLL Fault in Zone-2 & $0 / \mathrm{F}$ & $0 / \mathrm{F}$ & $0 / \mathrm{F}$ & $4 / \mathrm{T}$ & $4 / \mathrm{T}$ & $4 / \mathrm{T}$ & $0 / \mathrm{F}$ & $0 / \mathrm{F}$ & $0 / \mathrm{F}$ \\
\hline 2 & LG-A Fault in Zone-1 & $4 / \mathrm{T}$ & $0 / \mathrm{F}$ & $0 / \mathrm{F}$ & $0 / \mathrm{F}$ & $0 / \mathrm{F}$ & $0 / \mathrm{F}$ & $0 / \mathrm{F}$ & $0 / \mathrm{F}$ & $0 / \mathrm{F}$ \\
\hline 3 & LL-AC Fault in Zone-2 & $0 / \mathrm{F}$ & $0 / \mathrm{F}$ & $0 / \mathrm{F}$ & $4 / \mathrm{T}$ & $0 / \mathrm{F}$ & $4 / \mathrm{T}$ & $0 / \mathrm{F}$ & $0 / \mathrm{F}$ & $0 / \mathrm{F}$ \\
\hline \multicolumn{11}{|c|}{$50 \%$ DG Penetration } \\
\hline 1 & LLL Fault in Zone-2 & $0 / \mathrm{F}$ & $0 / \mathrm{F}$ & $0 / \mathrm{F}$ & $4 / \mathrm{T}$ & $4 / \mathrm{T}$ & $4 / \mathrm{T}$ & $0 / \mathrm{F}$ & $0 / \mathrm{F}$ & $0 / \mathrm{F}$ \\
\hline 2 & LG-A Fault in Zone-1 & $4 / \mathrm{T}$ & $0 / \mathrm{F}$ & $0 / \mathrm{F}$ & $0 / \mathrm{F}$ & $0 / \mathrm{F}$ & $0 / \mathrm{F}$ & $0 / \mathrm{F}$ & $0 / \mathrm{F}$ & $0 / \mathrm{F}$ \\
\hline 3 & LL-AC Fault in Zone-2 & $0 / \mathrm{F}$ & $0 / \mathrm{F}$ & $0 / \mathrm{F}$ & $4 / \mathrm{T}$ & $0 / \mathrm{F}$ & $4 / \mathrm{T}$ & $0 / \mathrm{F}$ & $0 / \mathrm{F}$ & $0 / \mathrm{F}$ \\
\hline \multicolumn{11}{|c|}{$100 \%$ DG Penetration } \\
\hline 1 & LLL Fault in Zone-2 & $0 / \mathrm{F}$ & $0 / \mathrm{F}$ & $0 / \mathrm{F}$ & $4 / \mathrm{T}$ & $4 / \mathrm{T}$ & $4 / \mathrm{T}$ & $0 / \mathrm{F}$ & $0 / \mathrm{F}$ & $0 / \mathrm{F}$ \\
\hline 2 & LG-A Fault in Zone-1 & $4 / \mathrm{T}$ & $0 / \mathrm{F}$ & $0 / \mathrm{F}$ & $0 / \mathrm{F}$ & $0 / \mathrm{F}$ & $0 / \mathrm{F}$ & $0 / \mathrm{F}$ & $0 / \mathrm{F}$ & $0 / \mathrm{F}$ \\
\hline 3 & LL-AC Fault in Zone-2 & $0 / \mathrm{F}$ & $0 / \mathrm{F}$ & $0 / \mathrm{F}$ & $4 / \mathrm{T}$ & $0 / \mathrm{F}$ & $4 / \mathrm{T}$ & $0 / \mathrm{F}$ & $0 / \mathrm{F}$ & $0 / \mathrm{F}$ \\
\hline
\end{tabular}




\section{Chapter 8}

\section{MAS Service Restoration}

The proposed MAS logic uses the hybrid control philosophy that makes use of both centralized and decentralized concepts to restore the unfaulty sections of the system. The major advantage of the hybrid control is that it can achieve optimal restoration solution.

FA waits until the recloser or the $\mathrm{CB}$ is completely locked-out on a permanent fault and it receives fault isolation confirmation from a down-stream SSA to initiate the restoration process. As explained in the Chapter above, SSAs can autonomously detect the over current faults using the Fault Detection algorithm. And the fault isolation is achieved by coordinated communications among SSAs.

The first step in the restoration process is that the FA sends out command signals to all SSAs except faulted zone SSA to open-up their respective SSWs. Picking up multiple cold (unenergized) zones at once can lead to severe in-rush currents through substation transformer and can adversely impact its health. To mitigate this risk of endangering the substation transformers, the proposed MAS logic follows a sequential energizing process to restore the unfaulty portion of the faulted circuit. FA divides the faulty feeder into two super zones namely Super Zone-A (collection of fault-free zones upstream of fault zone) and Super Zone-B (collection of fault-free zones downstream of fault zone) and handles them individually to restore service.

FA sends communication to all the zone representative SSAs to respond with their pre-fault loading measurements (three phase quantities of currents, voltages, and net-loading values). SSAs 
communicate with their neighboring SSAs, TSAs, and PAs to compute the respective zone prefault loading information. FA also sends communication to all TSAs and PAs to respond with their pre-fault measurements (three phase quantities of voltage, current, load, and generation values). This collective information helps FA to restore power to each un-faulted zone of the Super ZoneA and Super Zone-B. The FA maintains two lists for the Non-Energized Zones (NEZ) of Super Zone-A and Super Zone-B, namely NEZ_A and NEZ_B. During the service restoration process the FA follows strict requirement of maintaining radial nature as most of the electric utilities operate their distribution system in a radial fashion. Loosing radial nature can greatly impact the existing protection coordination set-up of the distribution network. Figure 8-1 below shows a typical distribution feeder with super zones and agent placement.

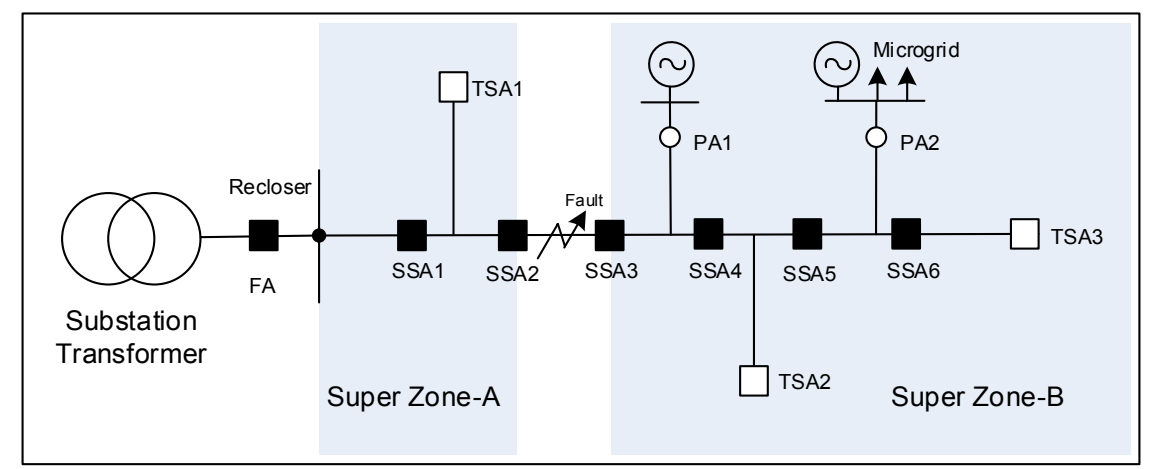

Figure 8-1: Typical Radial Distribution Feeder with Super Zones

After the above preparation, FA starts the restoration process for Super Zone_A. The FA energizes the first zone by sending a close command signal to the recloser/CB at the substation. After successfully energizing the first zone, FA updates the NEZ_A list by deleting the zone that it recently energized. FA energizes the next zone adjacent to the recently energized zone by sending a close command signal to the associated SSA. It again updates the NEZ_A list. This process repeats until all the NEZs in Super Zone_A are energized, or in other words the NEZ_A list is empty. The logic for restoring the Super Zone_A is shown in the following flow chart. 


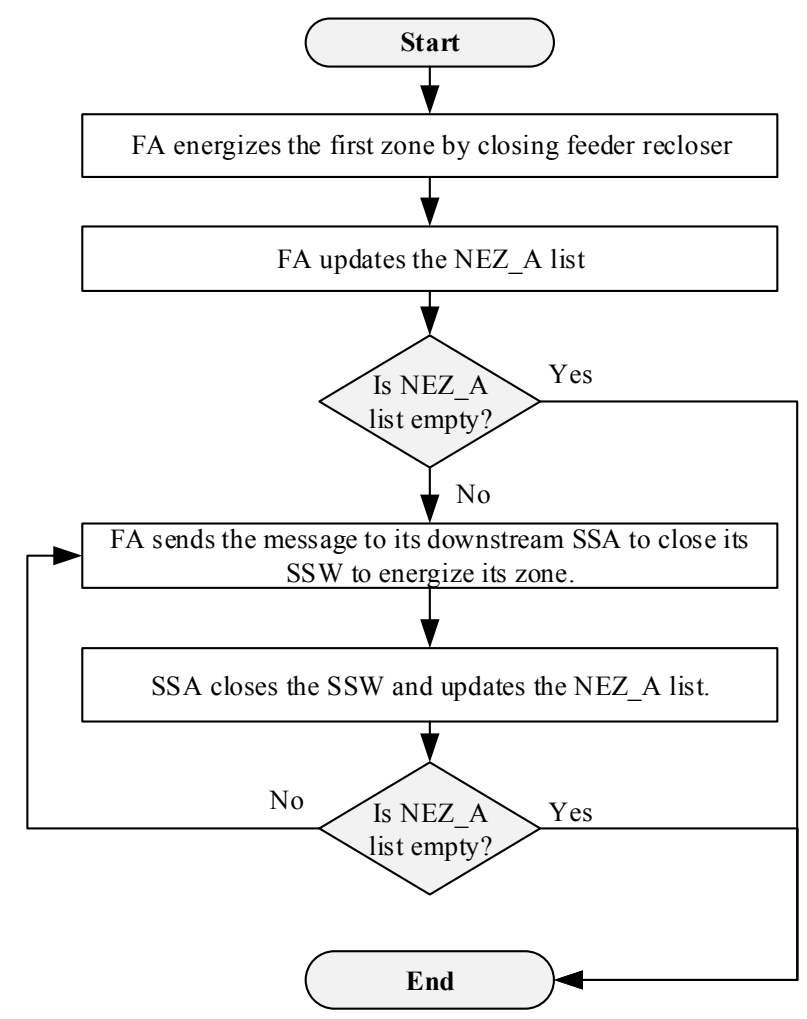

Figure 8-2: Service Restoration of Super Zone-A

\subsection{Restoration Formulation}

The service restoration problem for Super Zone-B with electrical, operational, and network constraints can be seen as a constrained multi-objective optimization problem. The objective functions enforce to restore power to as many loads as possible while maximizing the utilization of microgrids present in the distribution network, and also try to minimize the number of tie-switch operations to achieve the target restored network.

A feasible solution is one which satisfies the electrical constraints of KVL and KCL expressed as three-phase power flow equations. Also, branch current flows, feeder loading, substation transformer loading, and voltage magnitudes must lie within acceptable operating ranges. The final reconfigured network should retain the radiality of the system to keep the protection setup of the distribution network untapped. 
The mathematical formulation of the problem is shown below with its objective functions and constraints.

\section{Objective Functions:}

$$
\begin{gathered}
\max \left\{J_{1}=\sum_{i=1}^{n} \gamma_{i} * \beta_{i} *\left(Z L_{i}^{a}+Z L_{i}^{b}+Z L_{i}^{c}\right)\right\} \quad \forall i \in n \\
\max \left\{J_{2}=\sum_{i=1}^{n} \sum_{k=1}^{m} \mu G P_{i k}\right\} \quad \forall i \in n, \forall k \in m \\
\min \left\{J_{3}=t n_{o p s}\right\} \\
Z L_{i}^{p}=L F_{s s w(i+1)}^{p}+\sum_{i=1}^{n D G} D G_{i}^{p}-L F_{s s w(i+2)}^{p} \quad \forall i \in n D G, p \in a, b, c
\end{gathered}
$$

The fulfillment of the objective functions should be obtained by satisfying the following constraints.

1. Voltage Constraint:

$$
V_{i}^{\min } \leq V_{i}^{p} \leq V_{i}^{\max } \quad \forall i \in N, p \in a, b, c
$$

2. Branch Capacity Constraint:

$$
\left|I_{k}^{p}\right| \leq I_{k}^{\max } \quad \forall k \in M, p \in a, b, c
$$

3. Station Transformer Capacity Constraint:

$$
T k V A_{i} \leq T k V A_{i}^{n o m}
$$

4. Radiality Constraint:

$$
\sum_{j=1}^{n t} T S W_{i j} \leq 1
$$


Where,

$Z L_{i}^{a}$

$Z L_{i}^{b}$

$Z L_{i}^{c}$

$\beta_{i}$

$\gamma_{i}$

$n$

$m$

$\mu G P_{i k}$

$t n_{\text {ops }}$

$L F_{s S w(i+1)}^{p}$

$L F_{s S w(i+2)}^{p}$

$D G_{i}^{p}$

$n D G$

$N$

$V_{i}^{p}$

$V_{i}^{\min }$

$V_{i}^{\max }$

$M$

$\left|I_{k}^{p}\right|$

$I_{k}^{\max }$

$T k V A_{i}$

$T k V A_{i}^{\text {nom }}$

$T S W_{i j}$ pre-fault phase- $a$ net zone- $i$ load apparent power calculated by equation (8.4)

pre-fault phase- $b$ net zone- $i$ load apparent power calculated by equation (8.4)

pre-fault phase- $c$ net zone- $i$ load apparent power calculated by equation (8.4)

weight associated with zone- $i$ depending on its priority

binary value determining whether zone- $i$ should be restored or not (1: restored; 0 : not restored)

total number of zones to be restored

total number of microgrids available in zone- $i$

PCC status ( $0=$ Closed, $1=$ Open $)$ of microgrid $k$ in zone $-i$

number of tie-switch operations

pre-fault phase- $p$ apparent power flow through upstream SSW of zone- $i$

pre-fault phase- $p$ apparent power flow through downstream SSW of zone- $i$

pre-fault phase- $p$ apparent power output of DG- $i$

total number of DG units including the DGs available in the microgrids present in zone- $i$

set of all buses in the restored path

voltage magnitude of phase- $p$ at bus- $i$

minimum allowable voltage magnitude ( $0.95 \mathrm{pu}$ in our case study)

maximum allowable voltage magnitude (1.05 pu in our case study)

set of all branches in the restored path

current magnitude of phase- $p$ flowing through branch- $k$

continuous current carrying capacity of branch- $k$

apparent power flow through station transformer- $i$

nominal power rating of station transformer- $i$

status of $(1=$ Closed, $0=$ Open $)$ tie switch $-j$ in the extended zone- $i$ in the restored network

number of available tie switches in zone- $i$

The service restoration problem is an NP-complete problem with discrete and continuous variables, where the discrete variables are tie and sectionalizing switch statuses and the continuous variables are the network voltages and current flows. NP-complete problems typically require time consuming combinatorial optimization algorithms to solve for global optimal solutions [81]. Therefore, the service restoration problem is efficiently solved using the proposed algorithms, "Tie-Switch Ranking Algorithm" and the "Zone Priority Algorithm", in this research work. 
Agents in the proposed MAS perceive the system conditions in a continuous manner to learn and adapt to the new system conditions. Chapter 9 discusses the proposed reinforcement agent learning methodology used for the service restoration problem. Agent learning is archived in the "Agent Learning Database". Each time a successful FLIR action is taken by MAS the "Agent Learning Database" is updated with the MAS actions and pre and post system state. If there is no feasible restoration solution exists in the "Agent Learning Database", the concerned FA/SA performs the restoration optimization using the "Tie-Switch Ranking Algorithm" and the "Zone Priority Algorithm" to find out the best possible restoration plan for Super Zone-B.

The proposed MAS follows the logical steps explained in the flow chart shown in Figure 8-3 for restoring the fault-free zones of Super Zone-B. This logic uses the "Tie-Switch Ranking Algorithm" and the "Zone Priority Algorithm" that are explained in the following sections. One unique feature of this restoration logic is that it tries to make use of and account for existing DGs and microgrids in the restoration process.

The "net load" problem arises due to the presence of DG units on the systems, which means that the net load that needs to be supplied by the adjacent feeder Tie-switch is larger than the pre-fault load measurements. The anti-islanding protection trips DG units as soon as a fault condition appears on the system. And the current DER interconnection standards and regulations do not allow DGs to re-synchronize to the system until approximately five minutes following restoration of normal system. It causes the amount of load to transfer may exceed the amount measured just prior to the fault. The proposed logic accounts for the existing generation of DG units in a faultfree zone to compute the actual pre-fault net load.

On the other hand microgrids can stay in an islanded mode supplying the local load. It can help reduce the pre-fault load that needs to be transferred by the adjacent feeder. The PCC breaker is compliant with IEEE-1547 and UL-1741 anti-islanding standards that ensure that the breaker opens up as soon as there is a fault on the system to prevent an islanding condition. The breaker protection is designed to re-synchronize to the grid as soon as the power is restored. And it does not matter whether the power is restored in normal system configuration by the actual feeder or by 
an adjacent feeder. The proposed MAS makes sure that the PCC breaker is in open condition and thus the microgrid operates in islanded mode for the entire restoration process. This action helps to restore power to more fault-free zones by using same amount of adjacent feeder capacity.

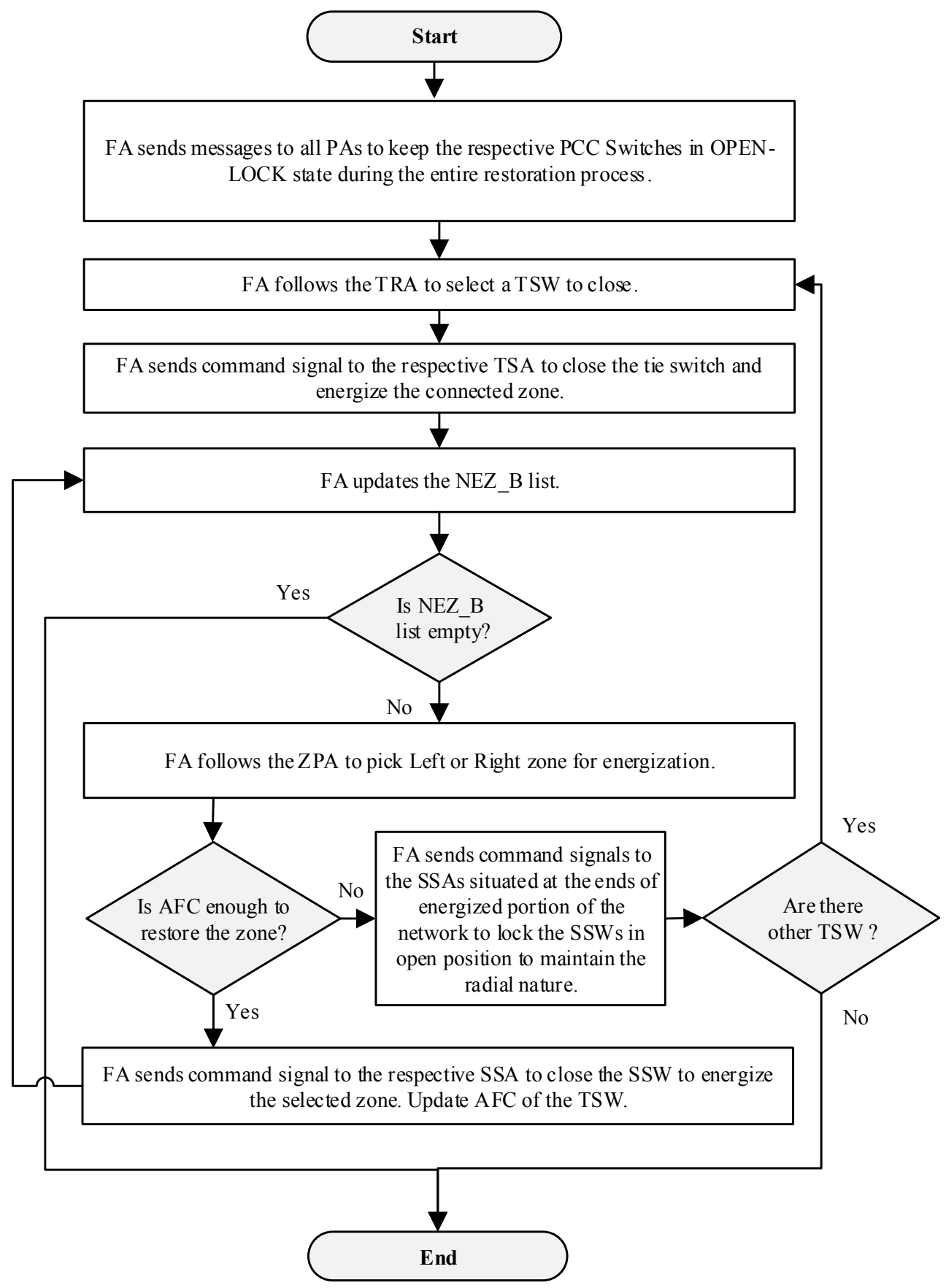

Figure 8-3: Service Restoration of Super Zone-B 


\subsection{TSW Ranking Algorithm (TRA)}

The algorithm is used by FA in prioritizing the TSWs to restore power to the fault-free zones of the network. The ranking of TSW is determined by two factors: one is the Type of TSW and the other is Available Feed Capacity (AFC) in kVA or amps. FA requests all of its TSAs to submit their AFC proposals to compute the TSW rankings. Figure 8-4 shows a typical configuration of a distribution system fed by two different substations indicating three different kinds of TSWs.

- Type-1: This TSW is placed between the feeders fed from same transformer of a substation.

- Type-2: This TSW is placed between the feeders fed from different transformers of the same substation.

- Type-3: This TSW is placed between the feeders fed from different substations.

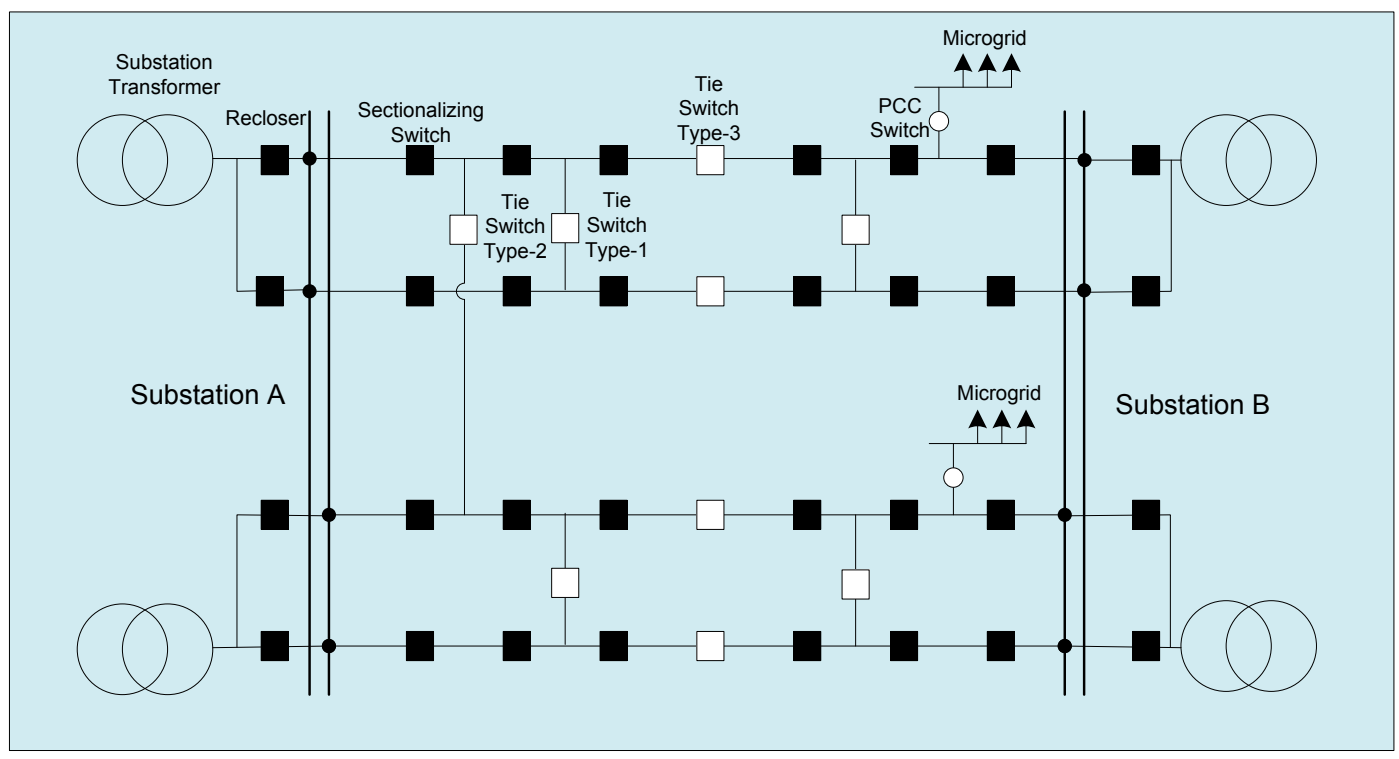

Figure 8-4: Typical Configuration of Distribution System

Usually, as a rule of thumb it is recommended to restore the fault-free zones using TSWs in the order of Type-1, Type-2, and Type-3. It is evident that using Type-1 TSW for reconfiguration will result in less line loss and better voltage profile configuration compared to Type-2 TSW. This is because the effective circuit length of reconfigured network using Type-2 TSW is more than the 
effective circuit length of reconfigured network using Type-1 TSW. Thus, the reconfigured network using Type-2 TSW will have more line losses and voltage drop compared to the reconfigured network using Type-1 TSW. Same holds good for Type-2 TSW and Type-3 TSW. Therefore, Type-1 TSW is given a higher priority compared to Type- 2 and Type-3 TSWs.

This algorithm also looks at AFC of TSW besides the "Type" information. AFC is defined as the load capacity in $\mathrm{kVA}$ or amps that can be served by a TSW without violating the system constraints as mentioned in the optimization formulation. AFC can be computed by running power flows either at the planning stage using the peak load values or during real time using exact net pre-fault loadings of the feeder zones after accounting for existing generation by DGs and microgrids. This is dictated by whether the FA/SA has the power flow computing capability or not.

Another simple way of computing AFC is by using the K-factors for computing the voltage drop along the restored circuit path. The $\mathrm{K}$-factor is the voltage drop percent down a line that is one mile long and serving a balanced three-phase load of $1 \mathrm{kVA}$. Where $l$ is the one way distance and $\mathrm{kVA}$ is the actual load of the line [82].

The voltage drop along a line segment is computed by:

$$
V_{\text {drop }}=K_{\text {factor }} * k V A * l
$$

One must note that AFC calculation using K-factors is an approximate calculation as the K-factor determination is based on several simplified assumptions that are seldom true for an actual distribution system. However, in this work we have used K-factors to calculate AFC values of the system besides the methods that uses power flow. As a future work, we identified the need to determine the impact of voltage regulating equipment such as shunt capacitor banks and voltage regulators on the standard K-factors. 
TSW ranking can be mathematically represented as shown below. The rank values are used to rank the available TSWs. The TSW with highest rank value is ranked number one in the list.

$$
R V\left(T S W_{i}\right)=A F C_{i} * W_{i}
$$

Where,

$R V\left(T S W_{i}\right) \quad$ Rank value of $T S W_{i}$

$A F C_{i} \quad$ Available Feed Capacity of $T S W_{i}$

$W_{i} \quad$ Weight factor of $T S W_{i}$

$W_{i}=1$ for Type-1 switch.

$W_{i}=0.9$ for Type- 2 switch.

$W_{i}=0.8$ for Type- 3 switch.

\subsection{Zone Priority Algorithm (ZPA)}

The algorithm is used by the FA to determine which fault-free zone to energize next following the recently energized fault-free zone using the same TSW. Figure 8-5 shows the fault-free zone energizing sequence by the ZPA algorithm. This algorithm uses the Left zone and Right zone terminology as defined below.

1) Left Zone: NEZ situated to the left side (upstream path towards substation source) of the most recent energized zone.

2) Right Zone: NEZ situated to the right side (downstream path towards the feeder end) of the most recent energized zone. 


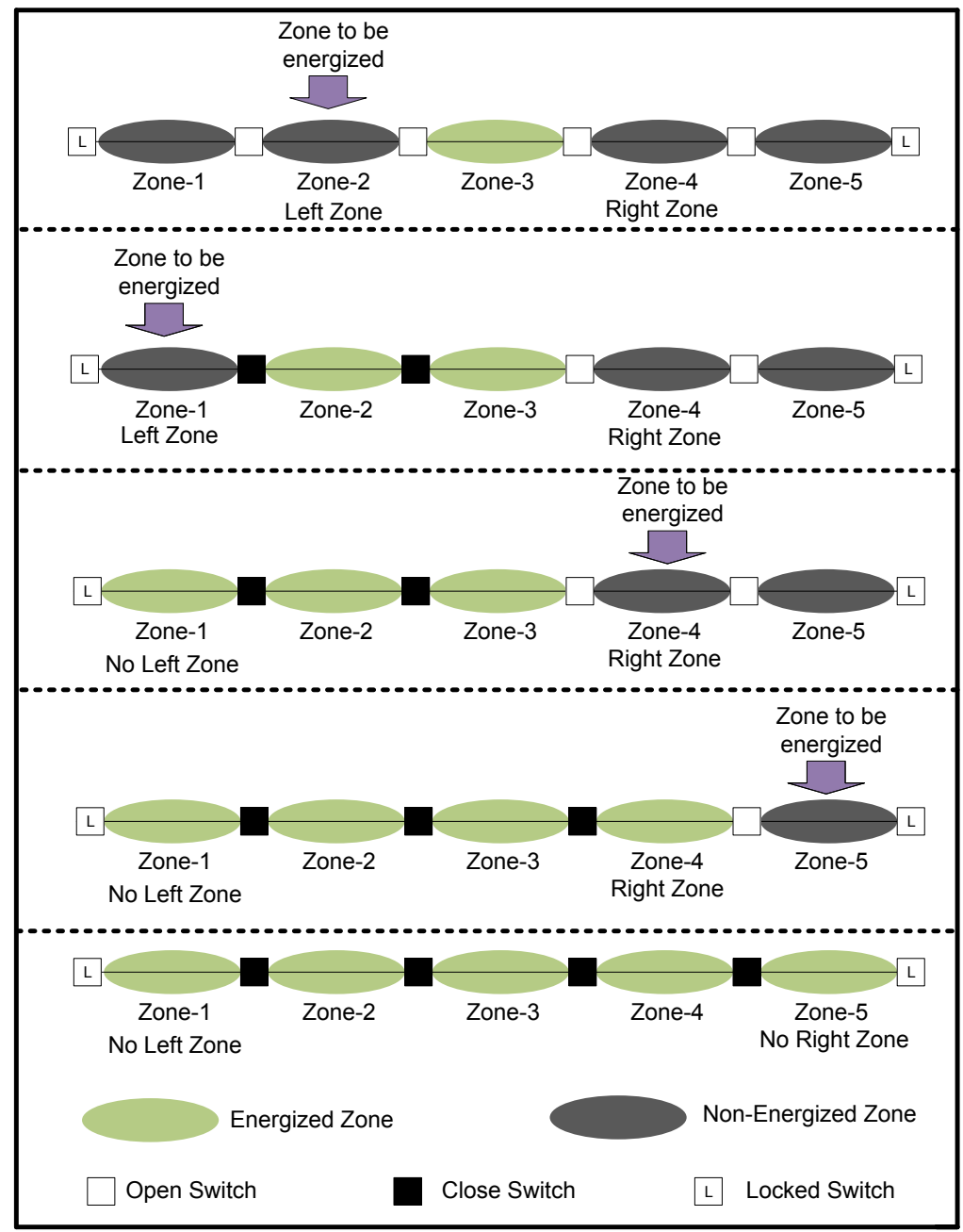

Figure 8-5: Zone Energizing Sequence using Zone Priority Algorithm.

This algorithm considers load priority of the zones. The zone that has important loads, such as hospitals, fire stations, universities, industries, and other commercial loads, is considered to be high priority zone compared to the other zones. Once a zone is energized, the next zone to be energized should be of higher priority zone between the Left and Right zones.

If the Left and Right zones carry same load priority, then FA picks the Left zone as default for energizing activity. This ensures maximum number of zones to be energized as the right most zones (downstream path towards feeder end) in Super Zone-B always have a TSW availability to back feed the feeder. If there is no Left zone i.e., if there is an open-locked SSW to the left side of the most recent energized zone then FA picks the Right zone for energizing activity. If there is no 
Right zone i.e., if there is an open-locked SSW to the right side of the most recent energized zone then FA skips to another TSW, if available, or stops the restoration process as NEZ_B list might be empty. All the zones in the WVSC test system are assumed to carry same load priority as per the guide lines from Mon Power. The guide lines are set by the Public Utility Commission (PUC) of Mon Power to treat each customer in the system with the same priority and to abide by the fairness rule.

\subsection{Multi-agent Logic for Return to Normal}

The system configuration needs to return to normal configuration once the fault in the system is fixed. This process is handled by the FA. FA waits for the Return to Normal command from the distribution operator, and as soon as it receives this command it starts communicating with its SSAs and TSAs to bring the system back to normal operating conditions.

FA communicates to TSAs, which have the closed TSWs that were used for restoration, to open the TSWs. Similarly, FA communicates with all PAs to lift LOCK condition on microgrid PCC switches. This ensures that PCC switch will be able to re-synchronize to the grid once the feeder configuration returns to normal and energized. FA communicates to all SSAs to open their SSWs to be prepared for the normal operation. Once FA receives confirmation from all TSAs, SSAs, and PAs, it sends command signal to close the recloser or circuit breaker to energize the first zone of the feeder. Once the zone is energized, FA will send the command signal to downstream SSA to close its SSW energizing its zone. FA repeats this procedure until all the zones are energized and system is brought back to normal configuration. The Return to Normal logic is shown in the following flowchart. 


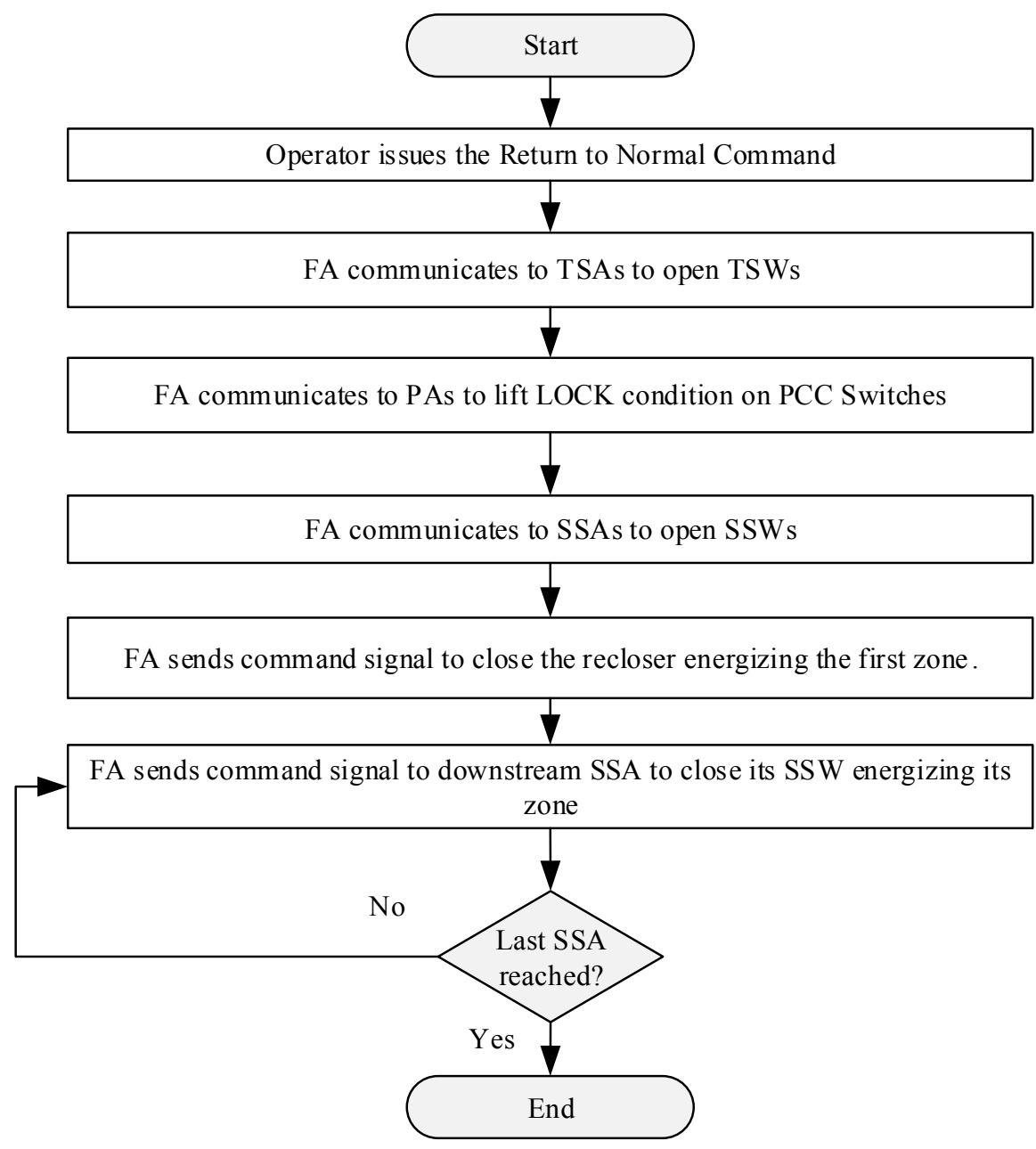

Figure 8-6: MAS Logic for Return to Normal 


\section{Chapter 9}

\section{Reinforcement Agent Learning}

The agents in a Multi-Agent System can be programmed with behaviors designed in advance, however, it is often necessary that they learn new behaviors online, such that the performance of the agent or of the whole Multi-Agent System gradually improves [83]. The static behaviors programmed in agents might not be sufficient to address all the issues of dynamic environment, and learning becomes a crucial aspect of Multi-Agent System design.

The agents learns by trial-and-error interactions with its dynamic environment using reinforcement learning. At each time step, the agent perceives the complete state of the environment and takes an action, which causes the environment to transit into a new state. The agent receives a scalar reward signal that evaluates the quality of this transition. The simplicity and generality of the setting makes RL attractive for Multi-agent learning.

Agent learning can greatly benefit the service restoration problem by providing reconfiguration solutions directly from its past experiences. Agents knowledge will be continuously updated online using the real-world experiences as the system takes action and receives the reinforcement signals from the system. The service restoration with RL can be realized with less number of communications among the agents and with minimal amount of time, hence improving the overall system reliability.

We used a famous reinforcement learning algorithm, $Q$-learning, for the agent learning of the service restoration problem. Being based on dynamic programming, this off-policy method 
theoretically can converge to the optimal reconfiguration [54] [41]. As $Q$-learning is an online approach, it can be deployed within a real electric distribution system, where the algorithm can not only fine-tune itself over time, but also re-adapt in the presence of non-stationary EDS environment. This makes $Q$-learning less susceptible to system drift due to configuration and equipment changes.

\subsection{Q-Learning}

$Q$-Learning algorithm is a reinforcement learning algorithm proposed by Watkins to deal with Markov Decision Process (MDP). In a MDP, the main objective of the agent is to find its action so as to maximize the sum of discounted expected rewards as given by the value function,

$$
V\left(s, \pi^{*}\right)=\max _{a}\left\{r(s, a)+\gamma \sum_{s^{\prime}} p\left(s^{\prime} \mid s, a\right) V\left(s^{\prime}, \pi^{*}\right)\right\}
$$

Where $r(s, a)$ is the reward for taking action $a$ to transition from the current state $s$ to next state $s^{\prime}, \gamma \in[0 ; 1)$ is the discount factor, $p\left(s^{\prime} \mid s, a\right)$ is the probability of transitioning into state $s^{\prime}$ after taking action $a$ at state $s$, and $\pi^{*}$ is the optimal strategy that satisfies the equation (9.1).

In $Q$-Learning agents do not know the reward function or the state transition probabilities, hence this is a form of model-free reinforcement learning. An action-value function or $Q$-function can be defined such that,

$$
Q^{*}(s, a)=r(s, a)+\gamma \sum_{s^{\prime}} p\left(s^{\prime} \mid s, a\right) V\left(s^{\prime}, \pi^{*}\right)
$$

$Q^{*}(s, a)$ is the total discounted reward of taking action $a$ at state $s$ and then following optimal policy thereafter. Comparing equations (9.1) and (9.2) we have,

$$
V\left(s, \pi^{*}\right)=\max _{a} Q^{*}(s, a)
$$


The problem of finding optimal policy $\pi^{*}$ is simply to identify the action that maximizes $Q^{*}(s, a)$. If we know $Q^{*}(s, a)$, we can find the optimal policy to take under any state. Therefore, the problem is finding $Q$-values for each state.

\subsubsection{Learning}

Agents maintain the $Q$-values for each and every possible state and action pair. Since the QLearning is an iterative method, the $Q$-values are initially assumed to be zero or some arbitrary small values. $Q$-learning provides a simple updating procedure to approximate the $Q$-value from empirical examples obtained from the actual experiences,

$$
Q_{t+1}\left(s_{t}, a_{t}\right)=(1-\alpha) Q_{t}\left(s_{t}, a_{t}\right)+\alpha\left[r_{t}+\gamma \max _{a} Q_{t}\left(s_{t+1}, a_{t}\right)-Q_{t}\left(s_{t}, a_{t}\right)\right]
$$

Where $\alpha \in[0 ; 1)$ is the learning rate which determines how much the new experiences affect the old $Q$-value. A factor of 0 will make the agent not learn anything, while a factor of 1 would make the agent consider only the most recent information. The learning rate is typically time varying and decreasing with time. Watkins and Dayan [56] proved that the sequence generated by (9.4) converges to $Q^{*}$ under the assumption that all states and actions have been visited infinitely often and the learning rate satisfies certain constraints.

\subsubsection{Exploration}

Exploration is the action selection mechanism selected by agent to transition into future states. There are three popular exploration techniques mentioned in the literature, $\epsilon$-greedy, Roulette, and Boltzmann distribution. The $Q$-values of any given state represents the effectiveness of the action, with higher values corresponding to better actions. Thus under a greedy policy, the best action to apply to a given state is simply,

$$
a=\operatorname{argmax}_{a^{\prime}}\left\{Q\left(s, a^{\prime}\right)\right\}
$$

As an immediate consequence of this greedy strategy, actions that are chosen in such a manner the agent reaches to its final state optimally. This is a desirable outcome, however, the greedy rule can 
obtain the best actions after the algorithm has explored a sufficient number of possible configurations and converged towards the actual $Q$-values. In order to explore the effectiveness of other actions during the learning stages of the algorithm, action selection must be carried out in a probabilistic manner. The Boltzmann distribution function to assign a probability $p\left(a \mid s_{t}\right)$ to the actions in order to create a graded function of estimated value:

$$
p\left(a \mid s_{t}\right)=\frac{\exp ^{Q_{t}\left(s_{t}, a\right) / \tau}}{\sum_{i=1}^{m} \exp ^{Q_{t}\left(s_{t}, a^{i}\right) / \tau}}
$$

The quantity $\tau$ is called the temperature parameter, which adjusts the randomness of action selection. An agent selects actions randomly when $\tau$ is high, and it selects actions based on greedy strategy when $\tau$ is low. Therefore, in order to make the algorithm exploratory, $\tau_{o}$ is initialized with a high value and decreased gradually towards a final low value $\tau_{\alpha}$. At the end of the predetermined number of iterations, the greedy strategy is applied to obtain a restoration action necessary to restore the maximum possible power with least number of switching operations.

\subsection{Learning Methodology for Service Restoration}

The main goal of Multi-Agent System using $Q$-learning is to restore power to as many fault-free zones as possible with minimum number of switching operations while utilizing available distributed generation and microgrids in the system. The agent has a learning module that consists of learning database, action selection module, reward module, and $Q$-updating module. As the name suggests, the learning database maintains $Q$-values for each possible state of the system, action selection module implements a specific strategy to select the best possible action that maximize the reward, reward module computes the scalar reward, and $Q$-update module updates the $Q$-values after each successful exploration of an episode.

\subsubsection{Reinforcement Reward Function}

Let the set of all possible states of the system be denoted as $S=\left\{s_{1}, s_{1} \ldots, s_{n}\right\}$ and the set of all actions available at a random state $s_{k}$ be denoted as $A_{k}=\left\{a_{k 1}, a_{k 1} \ldots, a_{k n}\right\}$. Each action entails 
either the opening or the closing of a tie switch or sectionalizing switch. The $Q$-learning is implemented in such a way that switching operations that cut off power to system zones are ignored. Therefore, in any state a smaller number of distinct actions in $A_{k}$ are explored by the algorithm.

The electric distribution system can be viewed as a Finite State Model (FSM). At any random state $s_{k}$, any available actions in $A_{k}$, transitions the system into a new state $s^{\prime}{ }_{k}$. The reinforcement signal or the reward function is then defined as an increment in the restoration objective function given by,

$$
\begin{gathered}
R\left(s_{k}, a_{k i}\right)=\Delta \mathcal{J}\left(s_{k}, a_{k i}\right)=\mathcal{J}\left(s^{\prime}{ }_{k}\right)-\mathcal{J}\left(s_{k}\right) \\
\mathcal{J}=\sum_{i=1}^{n} \gamma_{i} * \beta_{i} *\left(Z L_{i}^{a}+Z L_{i}^{b}+Z L_{i}^{c}\right) \quad \forall i \in n
\end{gathered}
$$

Where $\mathcal{J}$ is the service restoration objective function defined in section 8.1. The restoration action that would yield maximum power restored will have a large reward. The reward function in (9.7) can be modified to add additional restoration performance metrics such as percent voltage drop, line losses, and tie-switch operations. The modified reward signal is given by,

$$
R\left(s_{k}, a_{k i}\right)=\mathcal{W}_{j} *\left(\frac{P_{\text {restored }}}{P_{\text {outage }}}\right)+\mathcal{W}_{v d} *\left(1-\mathcal{V}_{\text {drop }}\right)+\mathcal{W}_{p l} *\left(1-\frac{\Delta P_{\text {loss }}}{P_{\text {feeder }}}\right)+\mathcal{W}_{\text {tn }} *\left(1-t * t n_{\text {ops }}\right)
$$

Where $P_{\text {outage }}$ is the total power outage due to the fault condition in the system, $P_{\text {restored }}$ is the amount of power restored, $\mathcal{V}_{\text {drop }}$ is the percent voltage drop calculated from feeder breaker to the end point of the final zone that is restored due to tie switch, $\Delta P_{\text {loss }}$ is the change in line loss for restored network before and after the restoration, $P_{\text {feeder }}$ is the feeder load of the restored network, $t=0.1$ is the scaling factor for number of tie switch operations, and $\mathcal{W}_{j}, \mathcal{W}_{v d}, \mathcal{W}_{p l}, \mathcal{W}_{t n} \in[0 ; 1]$ are the reward weights associated with performance parameters, load restoration, voltage drop, line loss, and number of tie-switch operations respectively. 
$\mathcal{W}_{j}, \mathcal{W}_{v d}, \mathcal{W}_{p l}, \mathcal{W}_{t n}$ weights can be configured in a way that we can prioritize agent learning with respective to any specific performance parameter or a combination of the parameters. In this work the weights are assumed as $\mathcal{W}_{j}=1, \mathcal{W}_{v d}=0.9, \mathcal{W}_{p l}=0.1, \mathcal{W}_{t n}=1$ to give the highest priority to restore power to as much load as possible using minimum number of tie switch operations while supplying good voltage profile. The agent learning is least impacted by line losses as it is a manageable operational parameter.

\subsubsection{Q-Value Matrix}

The feeder agent or the substation agent maintains the $Q$-value repository of all the information learned through its own experiences in the agent learning database. FAs can use the learning database for decision making and see how the past actions worked. The $Q$-value repository for the service restoration problem is a 2-D matrix and the size of the matrix depends on number of feeder zones, available tie switches, and sectionalizing switches. A typical $Q$-value matrix along with its associated switch combination matrix $\mathcal{H}_{F A_{h}}$ is given by,

$$
\begin{gathered}
\mathcal{Q}_{F A_{h}}\left(s_{k}, a_{k i}\right)=\left[\begin{array}{ccc}
\mathcal{Q}_{11} & \cdots & \mathcal{Q}_{1 z} \\
\vdots & \ddots & \vdots \\
\mathcal{Q}_{n 1} & \cdots & \mathcal{Q}_{n z}
\end{array}\right] \\
\mathcal{H}_{F A_{h}}\left(s_{k}, a_{k i}\right)=\left[\begin{array}{ccc}
\mathcal{H}_{11} & \cdots & \mathcal{H}_{1 x} \\
\vdots & \ddots & \vdots \\
\mathcal{H}_{n 1} & \cdots & \mathcal{H}_{n x}
\end{array}\right]
\end{gathered}
$$

Where $z$ is number of zones in the feeder $h$ monitored by the feeder agent $F A_{h}, x$ is total number of tie switches $x_{t}$ and sectionalizing switches $x_{s}$ present in feeder $h$ including the feeder breaker or recloser, and $n=2^{x}$ is number of possible switching combinations of $x$ number of tie switches, sectionalizing switches, and feeder breaker or recloser. $\mathcal{H}_{n x}$ is switch status index (1-close, 0 open) indicating the status of $x$ tie switch, sectionalizing switch, or feeder recloser. $\mathcal{Q}_{n z}$ represents the accumulated reward for $F A_{h}$ to choose the switching action specified in row vector of switch combination matrix $\mathcal{H}_{F A_{h}}=\left(\begin{array}{lll}\mathcal{H}_{n 1} & \cdots & \mathcal{H}_{n x}\end{array}\right)$ for service restoration of a fault in zone $z$. The $Q-$ 
value matrix is a sparse matrix since only a handful number of switching combinations are feasible for service restoration for a fault in a feeder zone.

For any state $s_{k}$, there is at least one action $a^{*}$ that has the largest reward and $Q$-value among the possible actions for a zone restoration.

$$
\mathcal{Q}\left(s_{k}, a^{*}\right)>\mathcal{Q}\left(s_{k}, a\right)
$$

\subsubsection{Q-Update Rule}

The $Q$-values in the matrix repository explained in the above section are updated after a successful implementation of an action using the update rule,

$$
Q_{t+1}\left(s_{t}, a_{t}\right)=(1-\alpha) Q_{t}\left(s_{t}, a_{t}\right)+\alpha\left[Q^{\prime}\left(s_{t}, a_{t}\right)\right]
$$

Where $Q$ and $Q^{\prime}$ are the last $Q$-value and the most recently obtained $Q$-value, $\alpha \in[0 ; 1)$ is the learning rate which determines how much the new experiences affect the old $Q$-value. A factor of 0 will make the agent not learn anything, while a factor of 1 would make the agent consider only the most recent information. We can make use of changing the learning rate to ensure the learning efficiency. The incremental change in $Q$-values, denoted by $\Delta Q$, should reduce progressively if $Q$ learning converges and conversely if $\Delta \mathcal{Q}$ is oscillating and diverges.

\subsubsection{Action Selection Strategy}

FA selects the switching actions based on the deterministic greedy strategy at a given state with $Q$-matrix values. If there are more than one switching solutions available, according to the greedy policy, the FA selects the switching action that maximizes the total reward,

$$
a=\operatorname{argmax}_{a^{\prime}}\left\{Q\left(s, a^{\prime}\right)\right\}
$$


The higher $Q$-values suggests to select the corresponding action with confidence. In the proposed service restoration $Q$-learning framework with the assumed weights and parameters, if $Q\left(s, a^{\prime}\right) \geq$ 2.85 means that the corresponding set of switching actions specified in switch combination matrix $\mathcal{H}_{F A_{h}}$ can restore power to all fault free zones with a voltage drop less than $5 \%$ and a line loss less than $5 \%$.

The greedy strategy obtains the best switching action choices if the algorithm has explored sufficient number of possible actions. If not, this action selection strategy might stay in a local optimum point. Therefore, it is imperative to explore all possible remedial actions using simulations to prevent the system trapping in local minimas.

\subsubsection{Q-Learning Process Flow}

The main advantage of the $Q$-learning is that the agents' knowledge will be continually updated online as the system takes action and receives the reinforcement learning signals from power system. However, the agents require initial knowledge of $Q$-values for possible events in the system before field deployment. The initial learning database can be built by off-line learning methodology through simulation models for each scenario. In this manner, Multi-Agent System will have a primary knowledge about scenarios it might encounter in the future.

The overall process flow of the proposed $Q$-learning approach is shown in Figure 9-1. FA stores the $Q$-values and corresponding switch combination matrix $\mathcal{H}_{F A_{h}}$ entries in memory for events that have been encountered previously, or for events that might likely to happen in future using off-line learning approach.

For a fault in EDS, FA looks into agent learning database to see if $Q$-values are present. If so, the optimal action based on greedy strategy in equation (9.14) is selected to perform switching actions specified in corresponding switch combination matrix $\mathcal{H}_{F A_{h}}$. The EDS returns the reinforcement

obtained by applying these switching actions based on the reward function in equation (9.9). And the stored $Q$-values are updated as mentioned in equation (9.13) and the state is updated to represent the current state. In this manner the $Q$-values can benefit from continual online updates using the sequence of reinforcement signals obtained directly from the EDS. 
If learning is not present for a fault scenario, the service restoration is solved using the optimization approach with TRA and ZPA algorithms. Once the solution is implemented in EDS, the $Q$-values for this particular scenario will be initialized in the FA's memory.
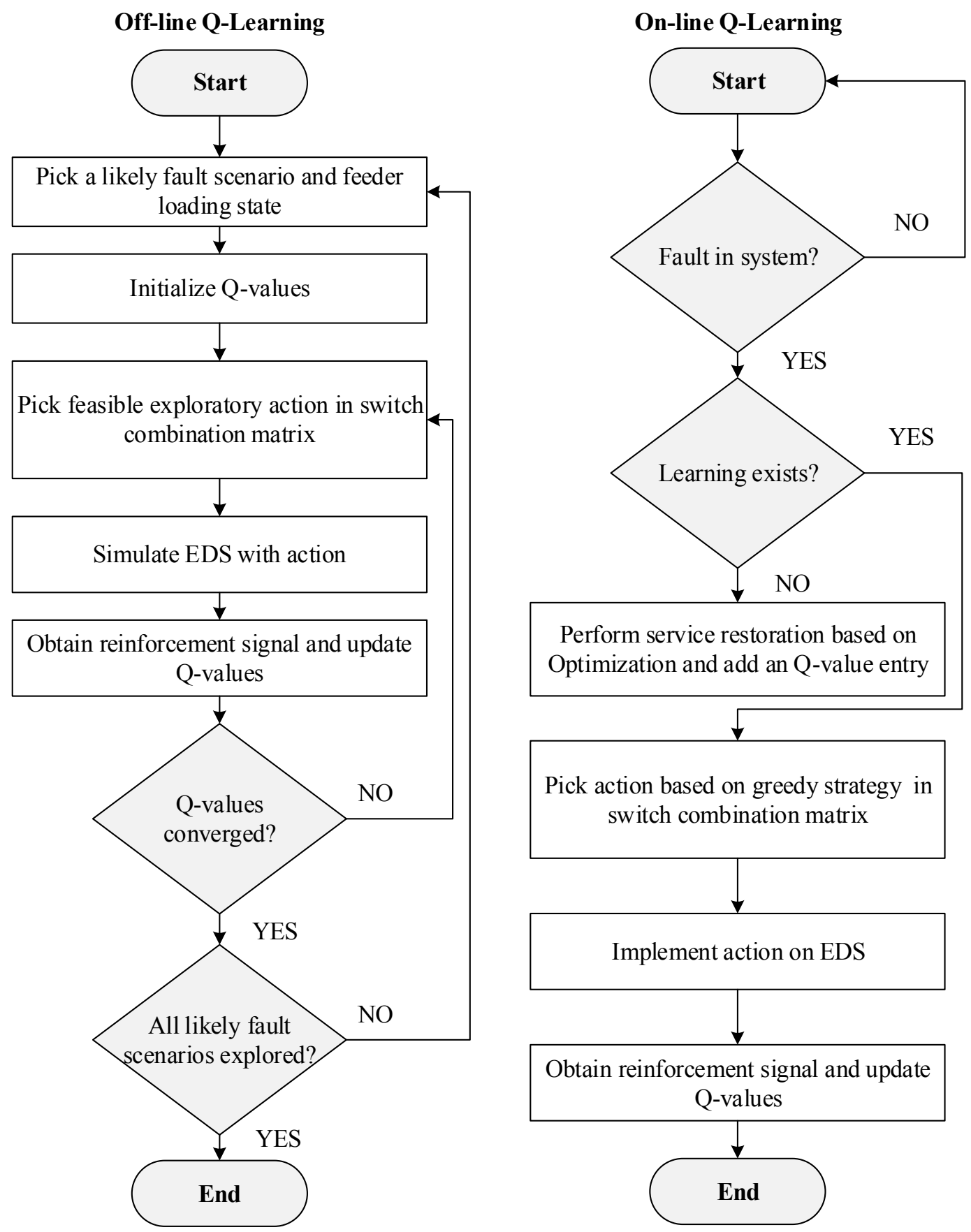

Figure 9-1: $Q$-Learning Process Flow for Service Restoration 


\section{Chapter 10}

\section{Service Restoration Results}

The distribution system associated with WVSC project is used to test the proposed MAS for Service Restoration. The WVSC distribution system is simulated by using MATLAB ${ }^{\circledR}$ Simpower $^{\circledR}$ toolbox and also $\mathrm{CYME}^{\circledR}$ for voltage drop studies, all agents are implemented using S-functions in Simulink. This simulation set-up needs no interface between MAS and power system model. This advantage provides a simpler and more accurate simulation model. During the simulation, Sfunctions use a special calling syntax called the S-function API that enables you to interact with the Simulink engine and synchronize the simulation of agents with power system model.

\subsection{WVSC System (Base Case)}

Figure 7-5 shows the $12.5 \mathrm{kV}$ distribution network of WVSC project fed from two substations WestRun and Pierpont. As mentioned in Chapter 7, the feeders WR\#3 and WR\#4 have Distribution Automation with 16 automated switches and 2 reclosers installed. The study concerns total of six feeders originating from WestRun substations and two feeders originating from Pierpont substation. Table 10-1 provides the peak load data for these feeders that are used to run the load flow studies for the base case. 
Table 10-1: Peak Load Data of WVSC Feeders

\begin{tabular}{cccccccccccc}
\hline & \multicolumn{2}{|c|}{ Phase-A } & \multicolumn{2}{c|}{ Phase-B } & \multicolumn{2}{c|}{ Phase-C } & \multicolumn{2}{c}{ Total } \\
\hline Feeder Name & kW & kvar & kW & kvar & kW & kvar & kW & kvar \\
\hline \multicolumn{8}{c}{ WestRun Substation } \\
\hline WR\#3 Stewart Street & 1917 & 747 & 1467 & 455 & 1865 & 697 & 5248 & 1898 \\
WR\#4 Pine View & 1381 & 198 & 1668 & 356 & 1668 & 363 & 4717 & 917 \\
WR\#5 Van Voohris & 432 & 218 & 379 & 191 & 377 & 190 & 1188 & 599 \\
WR\#7 University Ave & 844 & -25 & 1192 & 168 & 726 & -87 & 2762 & 56 \\
WR\#8 Mon Hospital & 677 & 134 & 550 & 69 & 442 & 16 & 1669 & 219 \\
WR\#1 Aspen Street & 249 & 121 & 358 & 175 & 148 & 71 & 755 & 367 \\
\hline \multicolumn{8}{c}{ Pierpont Substation } & & & \\
\hline PP\#5 Eaton & 1395 & -133 & 1665 & 27 & 1429 & -111 & 4489 & -217 \\
PP\#6 Mile Ground & 1037 & -15 & 774 & -162 & 1237 & 85 & 3047 & -92 \\
\hline
\end{tabular}

\subsection{WVSC System (Service Restoration Case)}

WR\#3 feeder is considered as a test system to prove the proposed MAS service restoration concept. WR\#3 feeder has 3 normally closed load break SSWs creating 4 zones and 5 normally open TSWs. For service restoration simulation purpose four DGs including two synchronous generators, one PV inverter, and one induction generator, and two microgrids are introduced into WR\#3 circuit as shown in Figure 10-1. All DGs and microgrids have associated PCC breakers that are completely complaint with IEEE-1547, i.e., when an islanding condition on the grid occurs, the DG and microgrid protection opens the PCC breakers within two seconds of the event to prevent unintentional islanding [79]. 


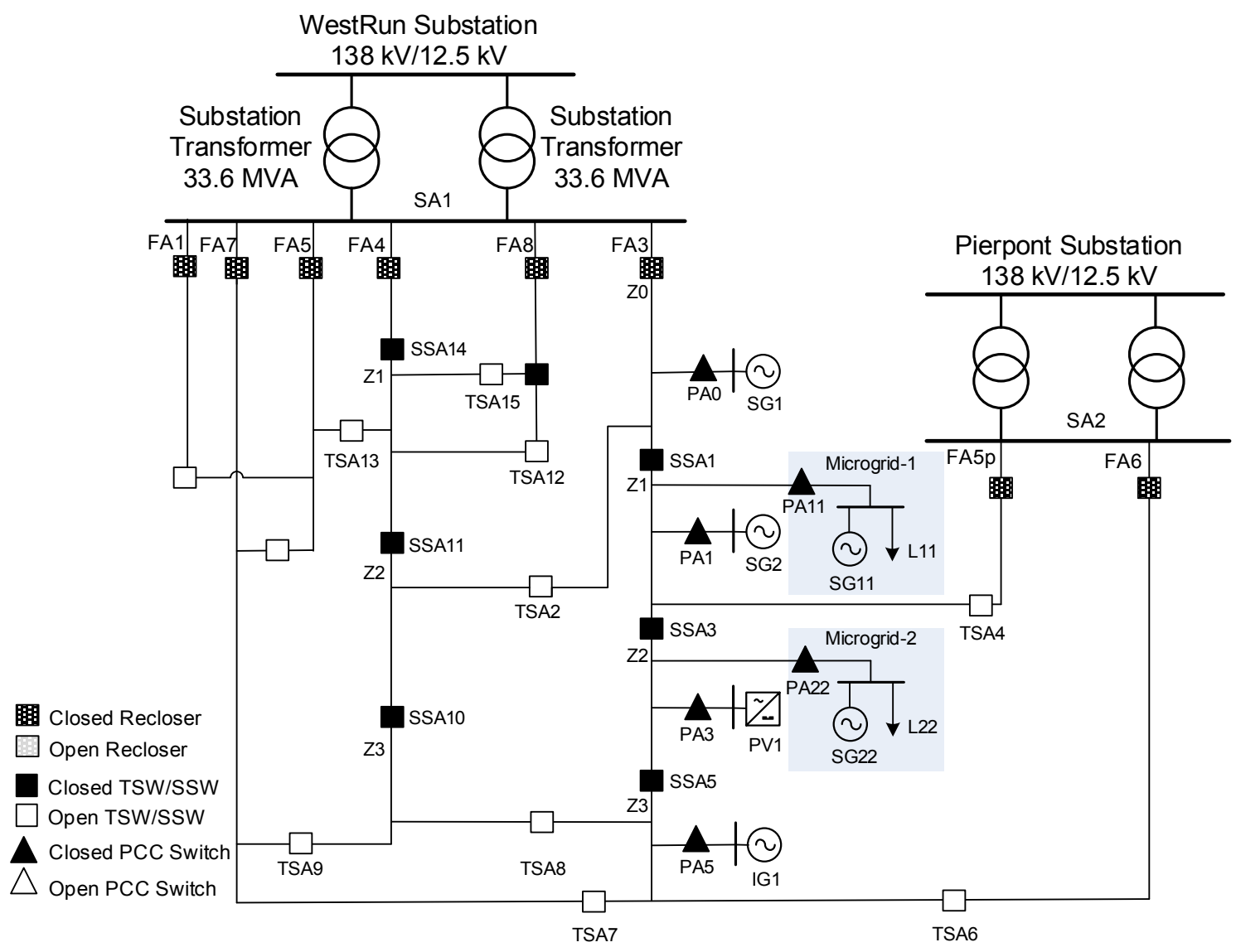

Figure 10-1: Simplified One Line Diagram of WVSC Feeders for MAS Service Restoration

\subsection{Power System Model}

Figure 10-2 shows the Matlab $^{\circledR}$ Simulink $^{\circledR}$ model and CYME $^{\circledR}$ model for WVSC feeders. Simulation results are calculated based on per unit values. The base MVA and voltage values considered for the simulation are $6 \mathrm{MVA}, 12.5 \mathrm{kV}$. Distribution lines are modeled based on the positive, negative, and zero sequence impedance values. Feeder loads are modeled with active and reactive power. The simulations are carried out in a discrete mode with a discrete step simulation time of 0.001 seconds. This results in 16 samples per cycle simulation readings which match with measured data resolution of actual automated switch hardware. 


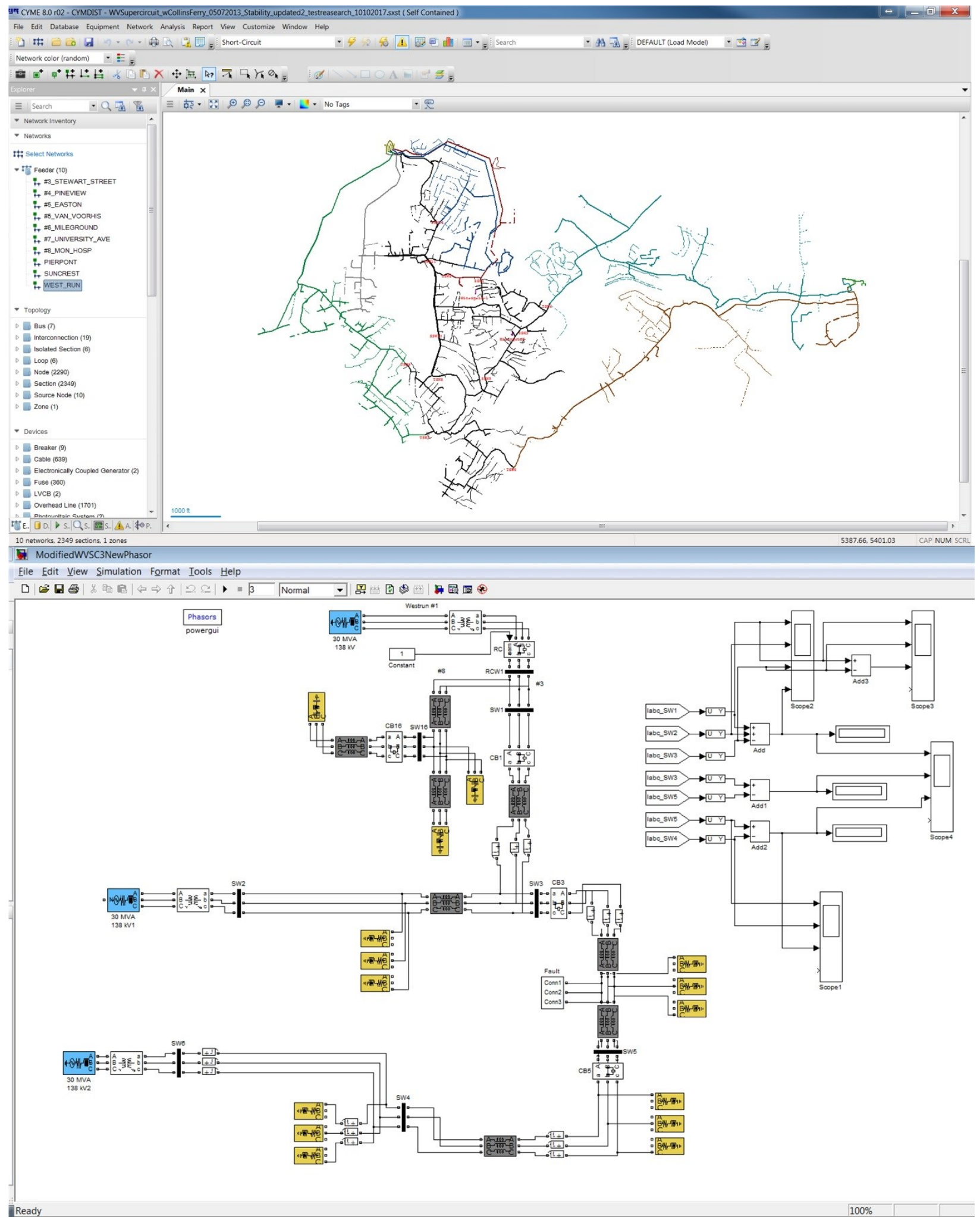

Figure 10-2: CYME ${ }^{\circledR}$ and Matlab ${ }^{\circledR}$ Model for WVSC Project Feeders 


\subsection{MAS Model}

The Multi-Agent System is implemented using the S-function blocks of MATLAB ${ }^{\circledR}$ Simulink $^{\circledR}$. Each agent is modeled by an S-function. S-functions (system- functions) provide a powerful mechanism for extending the capabilities of the Simulink R environment. Since the MAS is mainly including agents communication, and communications are intrinsically discrete in time, MAS works in discrete mode and it also provide the base to model communication delays and latency. The SSAs have access to voltage and current measurement data at the resolution of 16 samples per cycle. The agents communicate using the FIPA standard.

\subsection{Service Restoration Results}

The proposed MAS service restoration approach has been tested against various fault conditions. We are only demonstrating two typical samples, a three phase fault in Zone-0 of WR\#3 feeder and a single phase fault in Zone-2 of WR\#3 feeder.

\subsubsection{Case I - Fault in Zone-0}

In order to present service restoration results using the learning database and optimization approaches, we assume initially there is no agent learning available for this particular scenario. Therefore, the service restoration problem is first solved using the proposed optimization approach involving TRA and ZPA algorithms.

\subsubsection{Optimization Approach}

In this scenario a permanent three phase fault is considered in Zone- 0 of WR\#3 feeder for peak feeder loading state. The fault is isolated by FA3 and SSA1, and FA makes sure all the SSWs in WR\#3 feeder are open and the circuit is ready for service restoration. The circuit is divided into two super zones, Super Zone-A with all upstream zones of the faulted zone (Z0) and Super ZoneB with all downstream zones $(Z 1, Z 2$, and Z3) of the faulted zone (Z0). Since there are no zones without power in Super Zone-A, the restoration approach for Super Zone-A mentioned in Chapter 8 is not required. The Super Zone-B restoration is carried out using the optimization approach involving TRA and ZPA algorithms. 
Table 10-2 tabulates per-phase generation and net pre-fault loading values of each zone of the WR\#3 circuit as modeled in $\mathrm{CYME}^{\circledR}$. These values are compared against the Matlab ${ }^{\circledR}$ Simpower $^{\circledR}$ results for consistency. Table 10-3 tabulates TSW ranking calculated using the TRA.

Table 10-2: Zone Pre-fault Loadings and Generation Values in CYME ${ }^{\circledR}$

\begin{tabular}{c|cc|c|c|c|c|c|c|c|c}
\hline \multirow{2}{*}{$\begin{array}{c}\text { Zone/Generator } \\
\text { Name }\end{array}$} & \multicolumn{2}{|c|}{ Phase-A } & \multicolumn{2}{c|}{ Phase-B } & \multicolumn{2}{c|}{ Phase-C } & \multicolumn{3}{c}{ Total } \\
\cline { 2 - 10 } & $\mathbf{k W}$ & $\mathbf{k v a r}$ & $\mathbf{k W}$ & $\mathbf{k v a r}$ & $\mathbf{k W}$ & $\mathbf{k v a r}$ & $\mathbf{k W}$ & $\mathbf{k v a r}$ & $\mathbf{k V A}$ \\
\hline \multicolumn{10}{c}{ WR\#3 Stewart Street } \\
\hline Z0 & 106 & 117 & 107 & 80 & 208 & 144 & 422 & 341 & 543 \\
Z1 & 459 & 241 & 528 & 279 & 817 & 437 & 1804 & 956 & 2042 \\
Z2 & 476 & 257 & 262 & 145 & 177 & 97 & 915 & 498 & 1042 \\
Z3 & 875 & 132 & 570 & -49 & 662 & 19 & 2107 & 103 & 2110 \\
SG1 & 100 & 0 & 100 & 0 & 100 & 0 & 300 & 0 & 300 \\
SG2 & 150 & 0 & 150 & 0 & 150 & 0 & 450 & 0 & 450 \\
PV1 & 50 & 0 & 50 & 0 & 50 & 0 & 150 & 0 & 150 \\
IG1 & 75 & 0 & 75 & 0 & 75 & 0 & 225 & 0 & 225 \\
SG11 & 400 & 0 & 400 & 0 & 400 & 0 & 1200 & 0 & 1200 \\
SG22 & 200 & 0 & 200 & 0 & 200 & 0 & 600 & 0 & 600 \\
\hline
\end{tabular}

Table 10-3: TSW Ranking Based on TRA

\begin{tabular}{c|c|c|c|c}
\hline $\begin{array}{c}\text { Switch } \\
\text { Name }\end{array}$ & $\begin{array}{c}\text { TSW } \\
\text { Type }\end{array}$ & $\begin{array}{c}\text { AFC } \\
\text { (kVA) }\end{array}$ & $\begin{array}{c}\text { Ranking } \\
\text { Value }\end{array}$ & $\begin{array}{c}\text { TSW } \\
\text { Ranking }\end{array}$ \\
\hline TSW2 & 2 & 4000 & 3600 & 2 \\
TSW4 & 3 & 3500 & 2800 & 3 \\
TSW6 & 3 & 1550 & 1240 & 5 \\
TSW7 & 2 & 1800 & 1620 & 4 \\
TSW8 & 2 & 4500 & 4050 & 1 \\
\hline
\end{tabular}

The steps involved in the service restoration of Z1, Z2, and Z3 are outlined below.

1. FA3 detects the fault right after the recloser trials, and FA3 and SSA1 isolates the fault.

2. FA3 sends REQUEST performative messages to SSA3 and SSA5 to open up and get prepared for the restoration.

3. SSA3 and SSA5 sends ACCEPT performative messages and open SSW3 and SSW5. 
4. FA3 sends $Q U E R Y$ REF performative messages to request net pre-fault loading from SSA1, SSA3, and SSA5.

5. FA3, SSA1, SSA3, and SSA5 computes net pre-fault loading of their respective zones by communicating with their neighboring SSAs, TSAs, and PAs.

6. SSA1, SSA3, and SSA5 sends INFORM performative messages providing net pre-fault loading information.

7. FA3 determines required switching actions based on TRA and ZPA algorithms.

8. FA3 sends REQUEST performative messages to PA11 and PA22 to open-up PCC breakers.

9. PA11 and PA22 sends ACCEPT performative messages to FA3 and keep the PCC breakers in Open-Lock position.

10. FA3 sends a REQUEST performative message to TSA8 to close TSW8.

11. TSA8 sends a $A C C E P T$ performative message after closing TSW8.

12. FA3 sends a REQUEST performative message to SSA5 to close SSW5.

13. SSA5 sends a $A C C E P T$ performative message after closing SSW5.

14. FA3 sends a REQUEST performative message to SSA3 to close SSW3.

15. SSA3 sends a $A C C E P T$ performative message after closing SSW3.

16. FA3 creates a $Q$-value entry in the agent learning database corresponding to this scenario, and ends the restoration process.

The proposed MAS agent communication for restoring the healthy portion of WR\#3 feeder is shown in Figure 10-3. 


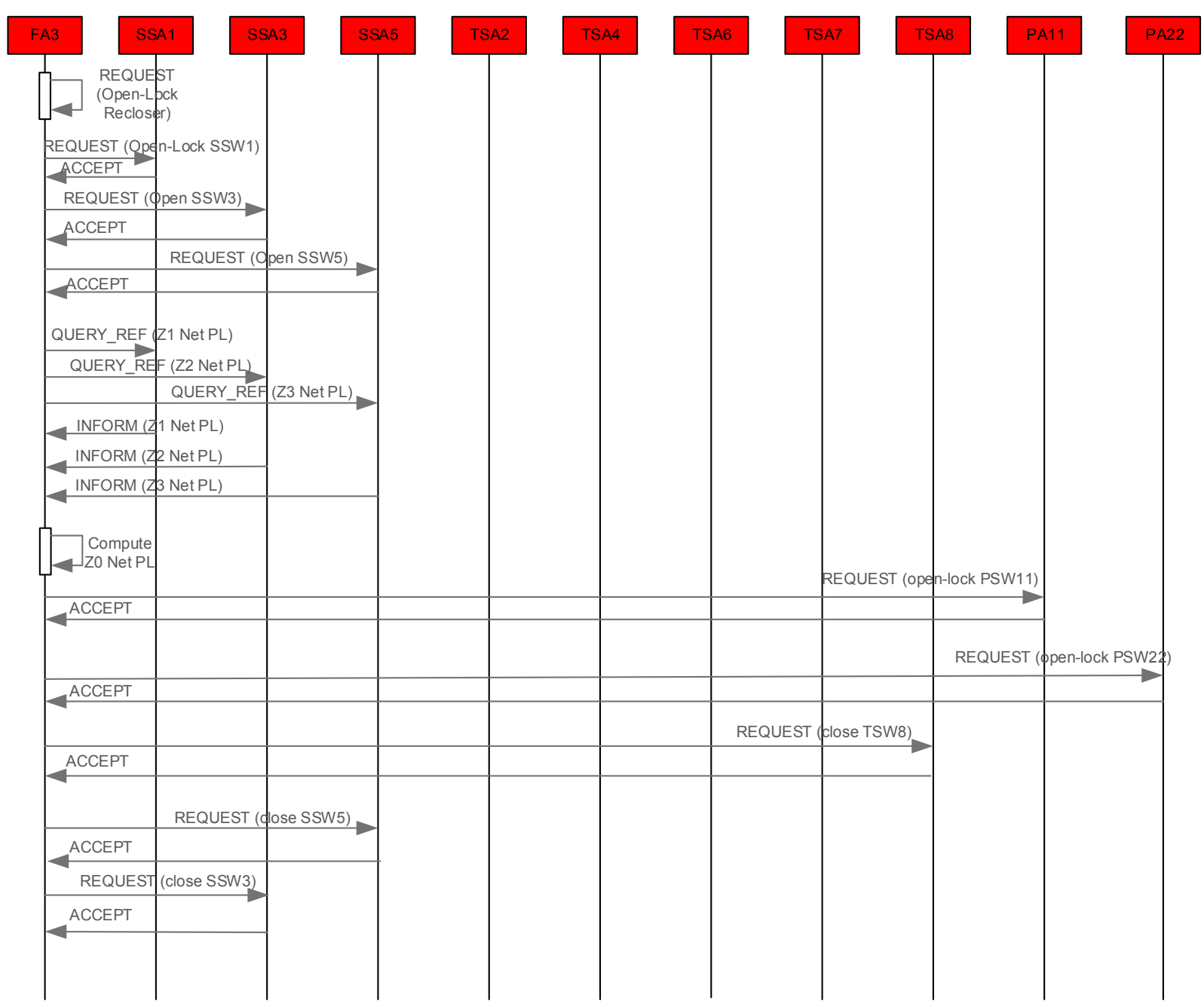

Figure 10-3: Agent Communications for Service Restoration for Zone-0 Fault

\subsubsection{Agent Learning Approach}

FA maintains the learning knowledge, the $Q$-values, for all the fault scenarios that have been encountered by the EDS in the past and the likely scenarios that might happen in the future. The $Q$-matrix $\mathcal{Q}_{\mathrm{FA}_{3}}$ and the associated switch combination matrix $\mathcal{H}_{\mathrm{FA}_{3}}$ computed through off-line learning using the model simulations at the feeder peak load state $s_{\text {peak }}$ for WR\#3 circuit are shown below. 


$$
\begin{aligned}
& \left.\begin{array}{cccccc} 
& & Z_{0} & Z_{1} & Z_{2} & Z_{3} \\
0 & \vdots & 0.00 & 0.00 & 0.00 & 0.00 \\
\vdots & \vdots & \vdots & \vdots \\
11 & 2.80 & 0.00 & 0.00 & 0.00 \\
74 & 2.78 & 0.00 & 0.00 & 0.00 \\
\mathcal{Q}_{F A_{3}}\left(s_{\text {peak }}\right)= & 82 & 2.77 & 0.00 & 0.00 & 0.00 \\
& 262 & 0.00 & 0.00 & 0.00 & 2.89 \\
265 & 0.00 & 2.87 & 0.00 & 0.00 \\
& 268 \\
272 & 0.00 & 0.00 & 2.79 & 0.00 \\
0.00 & 2.50 & 0.00 & 0.00 \\
276 & 0.00 & 0.00 & 2.77 & 0.00 \\
\vdots & \vdots & \vdots & \vdots \\
511 & 0.00 & 0.00 & 0.00 & 0.00
\end{array}\right] \\
& F R_{3}, T S W_{2}, T S W_{4}, T S W_{6}, T S W_{7}, T S W_{8}, S S W_{1}, S S W_{3}, S S W_{5}
\end{aligned}
$$

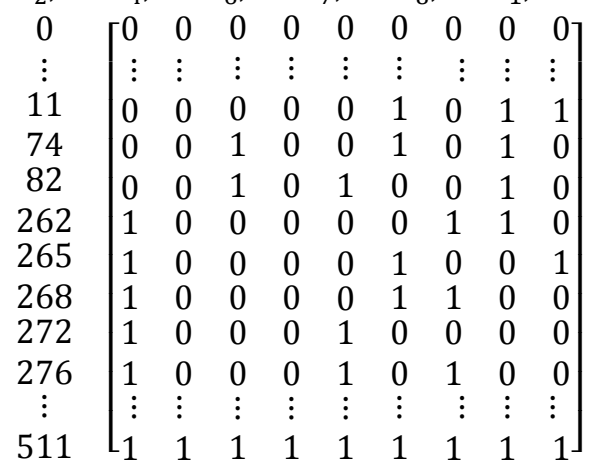

Since the WR\#3 circuit has 4 zones and 9 total number of tie and sectionalizing switches including the feeder recloser, the resulting sizes of $\mathcal{Q}_{F A_{3}}$ and $\mathcal{H}_{F A_{3}}$ matrices are 512X4 and 512X9. As explained before, the $Q$-value matrix is a sparse matrix since only a handful number of switching combinations are feasible for service restoration for a fault in a feeder zone. The columns of the $\mathcal{Q}_{\mathrm{FA}_{3}}$ represent $Q$-values for service restoration of faults in individual zones $\left\{Z_{0}, Z_{1}, Z_{2}, Z_{3}\right\}$ of WR\#3 circuit. The columns of the $\mathcal{H}_{F_{3}}$ represent switching action (1: close, 0 -open) of individual tie switches, sectionalizing switches, and feeder recloser available in WR\#3 Stewart Street circuit $\left\{\mathrm{FR}_{3}, \mathrm{TSW}_{2}, \mathrm{TSW}_{4}, \mathrm{TSW}_{6}, \mathrm{TSW}_{7}, \mathrm{TSW}_{8}, \mathrm{SSW}_{1}, \mathrm{SSW}_{3}, \mathrm{SS} W_{5}\right\}$.

For a fault in $Z_{0}$, the fault free zones $Z_{1}, Z_{2}, Z_{3}$ are restored using the set of switching actions specified in $\mathcal{H}_{\mathrm{FA}_{3}}$ correspoding to the maximum $Q$-value in the first column of the $\mathcal{Q}_{F A_{3}}$ based on the greedy strategy mentioned in equation (9.14). The maximum Q-value in the first column of $\mathcal{Q}_{F_{3}}$ is 2.80 which corresponds to row-12. This represents the degree of suggestion to select the switching actions specified in row-12 of $\mathcal{H}_{F A_{3}}$. Therefore, FA3 selects switching action vector 
$\{0,0,0,0,0,1,0,1,1\}$, which indicates to close $T S W_{8}, S S W_{3}, S S W_{5}$ in a sequential manner. FA3 communicates with respective agents $T S A_{8}, S S A_{3}, S S A_{5}$ to restore power to $Z_{1}, Z_{2}, Z_{3}$. Figure 10-4 shows the WR\#3 circuit after the service restoration for this scenario.

The $Q$-value for the recent transition should be updated using equation (9.13). The updated $Q$ value is larger if the taken actions restore more loads with improved voltage profile with less number of tie switch operations. The reinforcement reward of taking the action specified above results in value of 2.86, since the reconfigured system has less than 5\% voltage drop, less than $1 \%$ increase in line losses compared to normal system configuration, and one tie switch operation. The updated value for $\mathcal{Q}_{12,1}$ with the learning rate of 0.1 is increased to 2.81 , which shows an improvement in the confidence of taking the corresponding action. The updated $Q$-Matrix will be used for future decision makings and is shown below.

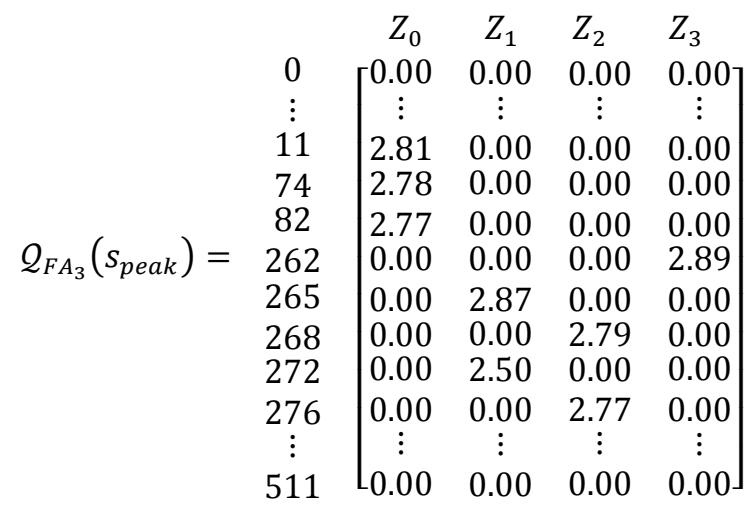

\subsubsection{Case II - Fault in Zone-2}

In this scenario a permanent single phase fault is considered in Zone-2 of WR\#3 circuit for peak loading state. The fault is isolated by SSA3 and SSA5, and FA makes sure all the SSWs in WR\#3 feeder are open and the circuit is ready for service restoration. The circuit is divided into two super zones, Super Zone-A with all upstream zones of the faulted zone (Z0 and Z1) and Super Zone-B with all downstream zones (Z3) of the faulted zone (Z2). The fault free zones in Super Zone-A ( $\mathrm{Z} 0$ and $\mathrm{Z1}$ ) are restored by closing the recloser following the Super Zone-A restoration approach mentioned in Chapter 8. The Super Zone-B restoration is carried out using the proposed agent learning approach since we assume there is sufficient agent learning available for this scenario. 
Since the fault is in $Z_{2}$, the maximum $Q$-value in the third column of $\mathcal{Q}_{F A_{3}}$ shown above is 2.79 which corresponds to row-269. This represents the degree of suggestion to select the switching actions specified in row-269 of $\mathcal{H}_{F A_{3}}$. Therefore, FA3 selects switching action vector $\{1,0,0,0,0,1,1,0,0\}$, which indicates to close $F R_{3}, T S W_{8}, S S W_{1}$ in a sequential manner. FA3 communicates with the respective agents $S S A_{1}, S S A_{5}$ to restore power to $Z_{0}, Z_{1}, Z_{3}$. Figure 10-4 shows the WR\#3 circuit after the service restoration for this scenario. The updated value for $Q_{269,3}$ with the learning rate of 0.1 is increased to 2.80 from 2.79. The updated $Q$-Matrix will be used for future decision makings and is shown below.

\begin{tabular}{|c|c|c|c|c|c|}
\hline & & $Z_{0}$ & $Z_{1}$ & $Z_{2}$ & $Z_{3}$ \\
\hline & 0 & $\lceil 0.00$ & 0.00 & 0.00 & 0.00 \\
\hline & 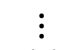 & $\vdots$ & $\vdots$ & $\vdots$ & $\vdots$ \\
\hline & 11 & 2.81 & 0.00 & 0.00 & 0.00 \\
\hline & 74 & 2.78 & 0.00 & 0.00 & 0.00 \\
\hline & 82 & 2.77 & 0.00 & 0.00 & 0.00 \\
\hline $\mathcal{Q}_{F A_{3}}\left(s_{\text {peak }}\right)=$ & 262 & 0.00 & 0.00 & 0.00 & 2.89 \\
\hline & 265 & 0.00 & 2.87 & 0.00 & 0.00 \\
\hline & 268 & 0.00 & 0.00 & 2.80 & 0.00 \\
\hline & 272 & 0.00 & 2.50 & 0.00 & 0.00 \\
\hline & 276 & 0.00 & 0.00 & 2.77 & 0.00 \\
\hline & $\vdots$ & $\vdots$ & $\vdots$ & $\vdots$ & $\vdots$ \\
\hline & 511 & $\mathrm{~L}_{0.00}$ & 0.00 & 0.00 & 0.00 \\
\hline
\end{tabular}

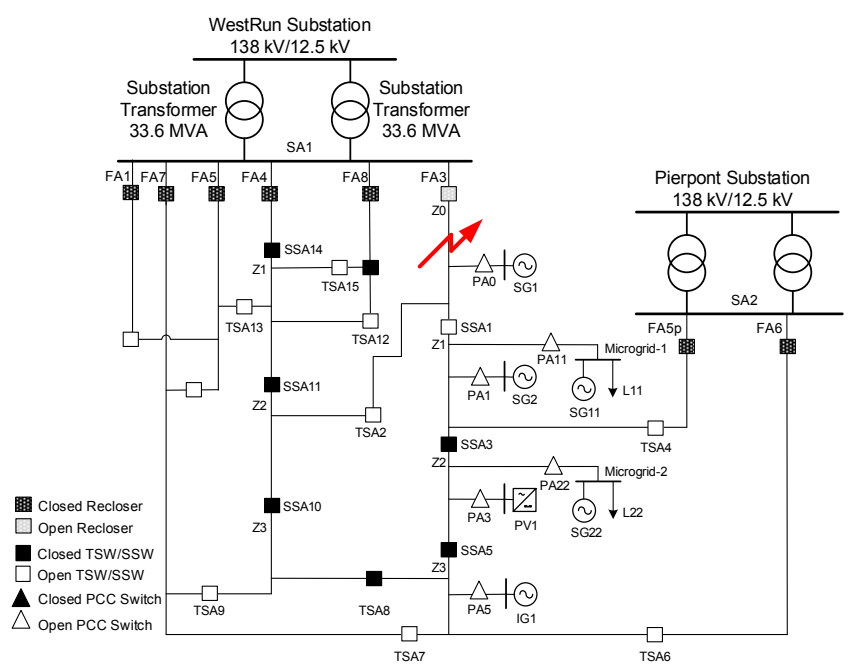

(A) Case I

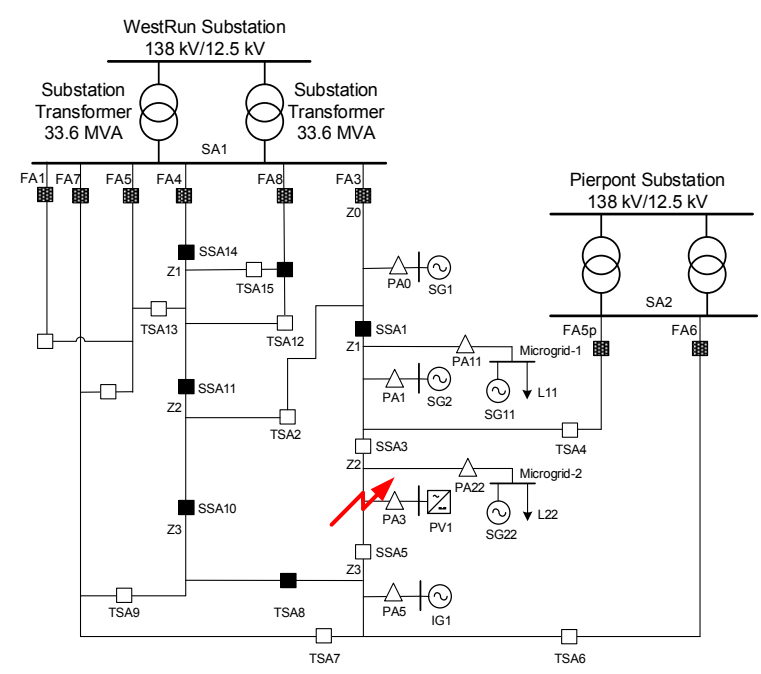

(B) Case II

Figure 10-4: Service Restorations for Case I and Case II 
The results presented show the efficacy of the proposed service restoration approach through mathematical optimization and agent learning. The proposed solution was able to identify suboptimal target configuration that can restore power to as many loads as possible with acceptable voltage profile and less number of tie switch operations while accounting for all DERs and microgrids present in the system. It should be noted that the proposed agent learning through $Q$ learning approach is an on-line method, in which the agent learning continues to improve as the MAS encounters different real-world events. The service restoration through agent learning would be a lot quicker as there are less number of agent communication acts and negotiations involved, hence contributing to improved system reliability.

\subsection{Performance Evaluation}

A key set of performance metrics listed below are identified in order to evaluate performance of the proposed MAS service restoration solution in terms of solution quality and speed. The proposed MAS service restoration solution is compared with other solution methods such as Centralized [46], Decentralized [45], and Hierarchical [52] as they present different control approach and solution architecture for the same problem.

\subsubsection{Agent Communications}

The restoration time is a significant performance index and the time consumed by agents to make restoration decisions matters as it counts towards the system reliability. The proposed hybrid MAS service restoration solution with the agent learning framework performs the service restoration with less number of agent communications than Centralized [46] and Decentralized [45] approaches.

The centralized service restoration approach generally needs an external omniscient central agent to maintain information about all agents in EDS. In addition, the central agent must be able to interact with all agents in the system. This approach has the potential of the single point of failure, i.e., if the central agent fails the entire system comes to halt. The service restoration approach proposed in [45] is a typical decentralized approach, where each agent has knowledge only about 
its neighboring agents. The agents acquire needed information through series of communications with the neighboring agents. As the system size increases, the agents will only have a limited view of the whole system and make relatively inaccurate restoration decisions. Therefore, often decentralized approaches fail to produce optimal solutions.

The proposed hybrid solution is a more balanced approach, which tackles decision making tasks in a centralized fashion, and all non-decision making tasks in a decentralized fashion. The restoration decision making in regards to which SSW, TSW, and PCC Switch to open or close happens at FA level. If for some reason the FA fails, the parent SA assumes the service restoration duties providing the necessary control redundancy. Since FA or SA has entire system view, the service restoration solution offered would be optimal.

The non-decision making tasks such as calculating individual zone net pre-fault loading are handled at the SSA level in a decentralized fashion, where the SSA communicate only with its neighboring SSAs, TSAs, and PAs. This decentralized approach can avoid hauling large amounts of data sets to FA or SA, which are usually situated far from the switching locations in EDS. In addition, it is likely to have less number of communication failures due to line of site issues if the agent communication is based on wireless communication.

The proposed MAS approach employs Q-learning in which the FA or SA learns in a continuous fashion from the past restoration solutions. Once the agent learning database matures, the FA can avoid unnecessary data acquiring communications, and restore the system with limited number of agent communications. This is a key advantage of the proposed solution, which not only leverages positive features of centralized and decentralizes approaches, but also integrates agent learning for service restoration.

Table 10-4 shows the number of required communication messages during the restoration case specified in section 10.5.1 (Fault in Z0 of WR\#3 Feeder) for Centralized [46], Decentralized [45], and proposed approaches. The assumption is that the fault is located and all the zones are isolated. Although WR\#3 feeder has only 4 zones, in order to show the impact of increasing system size on number of agent communications, the WR\#3 feeder is hypothetically modified to have increased 
number of zones and agents in Case-I and Case-II. It is evident that the proposed approach performs continuously better than the Decentralized and Centralized approaches in terms of number agent communications. The Decentralized approach incurs many number of communications due to the fact that agents not only carry their own communications, but also their neighboring agents' communications. It should be noted that the proposed MAS design requires less communication messages transmitted than other approaches because the communications are goal driven and learning knowledge helps the agents to prevent useless communication operations. Also, fewer number of communications means less delay and computational burden for agents, and can lead to a faster service restoration improving system reliability further.

Table 10-4: Number of Agent Communications

\begin{tabular}{|c|c|c|c|c|c|}
\hline \multirow{2}{*}{$\begin{array}{c}\text { Test Case } \\
\text { WR\#3 Z0 Fault }\end{array}$} & \multirow{2}{*}{$\begin{array}{c}\text { \# of } \\
\text { Zones }\end{array}$} & \multirow{2}{*}{$\begin{array}{c}\text { \# of } \\
\text { Agents }\end{array}$} & \multicolumn{3}{|c|}{ \# of Agent Communications } \\
\hline & & & $\begin{array}{c}\text { Centralized } \\
{[46]}\end{array}$ & $\begin{array}{c}\text { Decentralized } \\
{[45]}\end{array}$ & $\begin{array}{l}\text { Proposed } \\
\text { Approach }\end{array}$ \\
\hline Base Case & 4 & 16 & 16 & 35 & 15 \\
\hline Case-I & 8 & 31 & 39 & 162 & 32 \\
\hline Case-II & 16 & 61 & 81 & 679 & 66 \\
\hline
\end{tabular}

\subsubsection{Load Power Restoration}

The primary goal of the proposed MAS design is to restore power to as many loads as possible during emergency conditions. The proposed approach for service restoration integrates microgrids in to the solution. The advantage is that a tie-switch with same amount of available feed capacity can restore more number of loads. The available restoration solution in the literature Centralized [46], Decentralized [45], and Hierarchical [52] do not utilize available microgrids in the system.

Usually distribution feeders have several tie-points with adjacent feeders to restore power during emergencies. The test case in this dissertation, WR\#3 feeder, has 5 tie-switches connecting 4 adjacent feeders. In order to demonstrate the effectiveness of the proposed solution, the WR\#3 feeder is modified to have only one tie-switch TSW8. Table 10-5 presents the number of un-faulted zones restored and percentage of total outage power restored after a Z0 fault in WR\#3 feeder using Centralized [46], Decentralized [45], Hierarchical [52], and the proposed approach. As indicated 
all these approaches fail to restore power to Z1 of WR\#3 feeder, except the proposed approach. The total amount of power required to restore power to all un-faulted zones Z1, Z2, and Z3 is 5200 $\mathrm{kVA}$, whereas the available feed capacity of TSW8 is only $4500 \mathrm{kVA}$. The proposed approach utilizes microgrid-1 and microgrid-2 on WR\#3 feeder to offset the amount of required power by $1800 \mathrm{kVA}$. The solution approaches in Centralized [46], Decentralized [45], Hierarchical [52] can use load control to manage restoring power to all zones, however, that evaluation is not considered in this dissertation.

Table 10-5: Amount of Load Power Restoration

\begin{tabular}{c|c|c|c|c}
\hline \multirow{2}{*}{ Test Case } & \multicolumn{4}{|c}{ \# of un-faulted zones restored, \% of load power restored } \\
\cline { 2 - 5 } & $\begin{array}{c}\text { Centralized } \\
{[46]}\end{array}$ & $\begin{array}{c}\text { Decentralized } \\
{[45]}\end{array}$ & $\begin{array}{c}\text { Hierarchical } \\
{[52]}\end{array}$ & $\begin{array}{c}\text { Proposed } \\
\text { Approach }\end{array}$ \\
\hline WR\#3 Z0 Fault & $2,55 \%$ & $2,55 \%$ & $2,55 \%$ & $3,90 \%$ \\
\hline
\end{tabular}

\subsubsection{Tie-switch Operations}

The number of tie-switch operations is an important performance metric for automatic service restoration systems. Usually load break switches or reclosers are used for automatic sectionalizing of the feeder in DA projects. The number of operations of switching devices directly impact the life span, as each switching operation deteriorates insulation medium. The proposed service restoration approach is compared with Centralized [46], Decentralized [45], and Hierarchical [52] approaches. Although these methods are not applied for similar distribution system mentioned in this work, some assumption are made to facilitate the comparison.

The case specified in section 10.5.1, Fault in Z0 of WR\#3 Feeder, is used for the comparison. The proposed service restoration approach results in 1 tie-switch operation assuming that multiple zones can be restored up at the same time, whereas the Centralized approach also results in 1 tie switch operation. The systems tend to solve the problem locally and tend to result in multiple tieswitch operations, more likely one tie-switch operation for each zone restoration. The Hierarchical approach results in 2 tie-switch operations as the objective function does not include the criteria to minimize number of tie-switch operations. Table 10-6 presents the comparison of tie-switch operations for these approaches. 
Table 10-6: Tie-switch Operations

\begin{tabular}{c|c|c|c|c}
\hline \multirow{2}{*}{ Test Case } & \multicolumn{4}{|c}{ \# of Tie-Switch Operations } \\
\cline { 2 - 5 } & $\begin{array}{c}\text { Centralized } \\
{[46]}\end{array}$ & $\begin{array}{c}\text { Decentralized } \\
{[45]}\end{array}$ & $\begin{array}{c}\text { Hierarchical } \\
{[52]}\end{array}$ & $\begin{array}{c}\text { Proposed } \\
\text { Approach }\end{array}$ \\
\hline WR\#3 Z0 Fault & 1 & $>1$ & 2 & 1 \\
\hline
\end{tabular}

The proposed approach utilizes less number of tie switch operation to restore power to un-faulted zones. The TRA algorithm stops searching for other eligible tie switches until the existing tie switch exhausts all of its Available Feed Capacity (AFC). Other service restoration approaches in the literature try to utilize the tie switch available in each un-faulted zone, even if the tie switch has additional capacity to restore extra un-faulted zones. The performance can help with extending life span of automated switches, and can result in better overall economic performance of DA project.

\subsubsection{Line Losses}

Distribution line losses have direct impact on the system efficiency. The proposed TRA algorithm prioritizes the tie switches based on their type to account for electrical distance between adjacent feeder source to the tie switch. Therefore, it is likely the proposed service restoration logic can yield a reconfigured network with less line losses when compared to other service restoration mechanisms in literature that do not use electrical distance parameter in the restoration logic.

The test case mentioned in section 10.5.1, Fault in Z0 of WR\#3 Feeder, is used to illustrate line losses using different approaches. Table 10-7 presents a comparison of line losses of reconfigured network using Centralized [46], Decentralized [45], Hierarchical [52], and the proposed approach. Although the proposed approach likely to produce a target network with less line losses, it presents higher losses compared to other techniques in the given test scenario. This is due to the fact that the proposed solution has the objective to reduce number of tie-switch operations, and the proposed technique picks up all un-faulted zones with only one tie-switch operation. If the test case loading scenario is that where the proposed approach can restore each un-faulted zone with available tieswitch in the zone, the proposed approach results in less line losses. It is important to note that the 
main objective of service restoration problem should be the amount of load restored with minimal number of tie-switch operations and not line losses, since the service restoration is only for an emergency condition and it is a temporary event. The proposed MAS design observes this tradeoff between number of tie switch operations and line loss metrics, and gives priority to minimize number of tie-switch operations in its solution.

Table 10-7: Line Losses

\begin{tabular}{|c|c|c|c|c|}
\hline \multirow[b]{2}{*}{ Test Case } & \multicolumn{4}{|c|}{ Line Losses $(\mathrm{kW})$} \\
\hline & $\begin{array}{c}\text { Centralized }^{\mathbf{1}} \\
{[46]}\end{array}$ & $\begin{array}{c}\text { Decentralized }^{2} \\
{[45]}\end{array}$ & $\begin{array}{c}\text { Hierarchical }^{2} \\
{[52]}\end{array}$ & $\begin{array}{c}\text { Proposed } \\
\text { Approach }^{1}\end{array}$ \\
\hline WR\#3 Z0 Fault & 63.57 & 23.63 & 23.63 & 63.57 \\
\hline
\end{tabular}

\subsubsection{Voltage Drop}

As mentioned in the line losses section, since the proposed TRA algorithm utilizes electrical distance data in restoration formulation, the proposed service restoration logic can help with reducing the total voltage drop in the final target network. In other words, the proposed approach can help with providing a better end of line voltage compared to other restoration mechanisms in the literature that do not use electrical distance parameter in the restoration logic.

The test case mentioned in section 10.5.1, Fault in Z0 of WR\#3 Feeder, is used to illustrate voltage drop using different approaches. Table 10-8 presents a comparison of average voltage drop of reconfigured network using Centralized [46], Decentralized [45], Hierarchical [52], and the proposed approach. Although the proposed approach likely to produce a target network with less voltage drop, it presents higher voltage drop compared to other techniques because of the same reason mentioned in the line losses section. It is important to note that the proposed restoration provides a target reconfigured network with a voltage profile within acceptable levels mentioned in ANSI C84.1 standard. If the test case loading scenario is that where the proposed approach can restore each un-faulted zone with available tie-switch in the zone, the proposed approach results 
in less voltage drop. The proposed MAS design gives priority to minimize number of tie-switch operations over voltage drop metric as the event is a temporary one.

Table 10-8: Voltage Drop

\begin{tabular}{|c|c|c|c|c|}
\hline \multirow[b]{2}{*}{ Test Case } & \multicolumn{4}{|c|}{ Voltage Drop (\%) } \\
\hline & $\begin{array}{c}\text { Centralized }^{1} \\
{[46]}\end{array}$ & $\begin{array}{c}\text { Decentralized }^{2} \\
{[45]}\end{array}$ & $\begin{array}{c}\text { Hierarchical }^{2} \\
{[52]}\end{array}$ & $\begin{array}{c}\text { Proposed } \\
\text { Approach }^{1}\end{array}$ \\
\hline WR\#3 Z0 Fault & 4.67 & 2.88 & 2.88 & 4.67 \\
\hline
\end{tabular}

1. TSW8 is closed for restoration

2. TSW8 and TSW4 are closed for restoration 


\section{Chapter 11}

\section{Conclusions and Future Work}

\subsection{Conclusions}

In this dissertation, a novel hybrid Multi-Agent System with agent learning capability ( $Q$-learning) is proposed for electric distribution system automation with DER and microgrids. Also, an innovative economic model and algorithm is presented for the optimum switch number and placement problem of DA projects. The main contributions of this work are,

\section{- Hybrid Multi-Agent System Design}

A new hybrid MAS architecture for distribution automation system is proposed that is flexible and scalable. The proposed architecture makes use of both Centralized and Decentralized concepts to make up for the disadvantages of each method and achieve overall optimal solution. The proposed MAS architecture includes dedicated agents for distributed generators and microgrids to include those components into the FLIR solution. The agents can communicate with other agents in the same hierarchical level. However, the supervisory agents, feeder and substation agents, can exploit peer to peer communications with the lower level switch agents to reduce number of communications to speed up the FLIR processes. The decision making happens at the supervisory level, at the feeder agent or substation agent level, as these agents have the complete view of the system to achieve the optimal solution. 


\section{- Switch Optimization}

An innovative economic model is presented for the optimum switch number and placement problem of DA projects. The switch optimization is a complex and very important step in the economic feasibility process of any DA project. The proposed method finds the solution based on relative reduction in the normalized customer interruption costs. The main advantage of the proposed approach is that it isolates the impacts of varying switch investment and customer interruption cost data which is usually based on outdated utility survey studies. The proposed method is implemented on IEEE 34-bus, 123-bus, and WVSC test feeders. The mathematical model is developed in Matlab and the results show the computational robustness and efficacy of the solution. The proposed technique significantly scales down the search space and simplifies problem complexity that requires minimal computational effort and time.

\section{- Distributed Fault Location and Isolation}

Fault location and isolation is the first step of automatic FLIR systems, and faster FLI means faster service restoration and shorter outage time. Restoration process cannot be initiated unless the fault location is determined and faulty section is isolated. Therefore, it is important to locate and isolate the fault as fast as possible and preferably using a noncentralized approach.

A novel MAS FLI solution is presented for radial electric distribution systems with different types of DG resources which include synchronous, induction, and inverter-based resources. Unlike the traditional rotating machines, the fault response of an inverter based DG depends mostly on its control system rather than its physical parameters. The inverters are actively current limited due to the low thermal inertia of semiconductor switches, and thus have a small fault current contribution (1-2 pu) compared to conventional non-inverter based generators $(5-10 \mathrm{pu})$. Therefore, it is important to correctly account for DG fault characteristics in the FLI solution. Proposed FLI solution is one of the few available 
solutions that can locate the fault in electric distribution systems with DG penetrations without adding additional metering hardware.

The proposed FLI logic is totally distributed in nature that does not rely on centralized control approaches. An intelligent algorithm for Fault Detection is proposed, which can identify the faulted zone and also can determine the type of the fault. The sectionalizing switch agents can determine fault location autonomously based on the proposed Fault Detection Algorithm using their local data measurements. The fault isolation is achieved through a coordinated agent communication approach.

The proposed MAS FLI solution is illustrated by performing simulation studies on WVSC distribution system. A detailed PSCAD model of WR\#3 feeder is developed along with generator models to model a large scale inverter based DG, a synchronous generator, and an induction generator. The simulation platform is used to test the fault response of various types of DG resources for various types of faults in the system. The results show efficacy of the solution and authenticates the proposed MAS based FLI solution that works efficiently for radial distribution systems with different DG resources and with varying DG penetration levels.

\section{- Hybrid Service Restoration and Reconfiguration}

The proposed service restoration approach is based on a hybrid philosophy that utilizes both central and distributed control methods to make up for the disadvantages of each method and achieve an overall optimal solution. The proposed service restoration approach uses the "Tie-Switch Ranking Algorithm" and the "Zone Priority Algorithm" and makes use of available DG units and microgrids to find the best possible service restoration plan. The solution is formulated as a combinatorial multi-objective optimization to maximize amount of load restored with minimal number of tie-switch operations while maximizing available microgrids in the system. 
The proposed solution is tested on the real distribution system of WVSC project. The system include two substations and total of eight distribution feeders. The actual distribution network is simulated in $\mathrm{CYME}^{\circledR}$ and Matlab $^{\circledR} / \mathrm{Simpower}^{\circledR}$ whereas the MAS and its communications are simulated using Matlab ${ }^{\circledR}$ S-functions. The simulation results show promising advantages of using the proposed MAS solution for service restoration.

A key set of performance metrics are identified in order to evaluate performance of the proposed MAS service restoration solution in terms of solution quality and speed. The proposed MAS service restoration solution is compared with other solution methods in literature such as Centralized [46], Decentralized [45], and Hierarchical [52], as they present different control approach and solution architecture for the same problem. The evaluation revealed that the proposed approach results in less number of overall agent communications, hence faster service restoration, less number of tie-switch operations, and more number of un-faulted zone restored compared to the other methods. However, the proposed approach compromises on line losses and voltage drop metrics to meet other needed objectives of the service restoration.

\section{- Reinforcement Agent Learning}

Although the agents in MAS can be programmed with behaviors designed in advance, it is also necessary that they learn new behaviors online from their experiences, such that the performance of the agents and MAS gradually improves. In this dissertation, a modified reinforcement learning based on $Q$-learning is introduced to help agents in their decision making process. Although agents have the learning capability, they need to take actions to learn from them. In a real world power system fault events do not happen frequently and therefore it takes a long time for agents to gain knowledge about different scenarios. One possible solution to help agents learn faster is initializing agents' knowledge by simulating different possible scenarios in a software environment. 
Using the learning technology, agents in EDS will be expert decision makers over time and can make more accurate and comprehensive decisions for a self-healing distribution system.

\subsection{Future Work}

While this dissertation has discussed the design of a MAS for electric distribution system automation, many opportunities for extending the scope of this dissertation remain. The following main items can be suggested as future work:

\section{MAS application for Microgrids}

Although this dissertation has presented a MAS application for distribution automation to solve FLIR problems, there are many areas of power system where the MAS can be a key technology. MAS can offer modular, flexible, and integrated solutions for many complex issues of power systems. Microgrid is one such area, where MAS can provide distributed and autonomous solutions for real-time control and management.

The Smart Grid evolution is transforming the traditional distribution grids from passive to active networks due to the integration of microgrids and DER. Microgrids are among the most promising tools of modern distribution networks due to their versatility, which is demonstrated by the ability to simultaneously deliver enhanced reliability, security, energy and cost efficiency, as well as environmental benefits. The proliferation of microgrids and DER is making real-time control and system operations of traditional distribution grid more complicated. Therefore, smart control and energy management systems that are different from those commonly used in the past are necessary for efficient operation of modern power systems. The distributed nature and potential for modeling autonomous decision making entities in solving complex problems motivates the use of MAS for the control and management of microgrids. Proposed MAS has the autonomous decision making agents that can be modified for real-time control and management of a microgrid. Also, the MAS design can be extended for FLI within microgrids. 


\section{Switch optimization economic model}

Proposed economic model finds optimal number and location of automated sectionalizing switches based on relative reduction in the normalized customer interruption costs. The proposed model can be extended to form a comprehensive DA cost benefit frame work that can encompass all DA costs and benefits. The comprehensive model can include reliability improvements, in terms of SAIDI, CAIDI, and SAIFI indices, due to different switching technologies such as automated load break switches, reclosers, and sectionalizers.

The proposed method can be easily modified to accommodate other switching technologies such as reclosers and circuit breakers with various automation levels. Proposed approach cab be extended to include certain operational considerations such as back-feed capability without violating thermal loading and voltage constraints to arrive at the solution. Inclusion

of a distribution load flow to find the optimal number and placement of switches can be the immediate future work.

\section{Hardware implementation and testing}

Although proposed MAS solution for DA has been tested through simulation studies and the results are discussed, its application in a real world implementation can further prove the concept and provide additional insight into potential issues. There are various issues such as control and communication between agents and power system which are not always taken into consideration in the simulation studies. The proposed MAS DA solution is designed to be implemented on a real world distribution network as part of the DOE demonstration project, WVSC. The project ended as a design-only project, and the MAS solutions that were designed never got an opportunity to be fully implemented in the real distribution field. Development of a test system platform capable of emulating a present day power system and MAS will help in dealing with these challenges. 


\section{Bibliography}

[1] The U.S Department of Energy (DOE), [Online]. Available: http://energy.gov/oe/services/technology-development/smart-grid.

[2] "A vision for the modern grid," National Energy Technology Laboratory (NETL), [Online]. Available: http://www.netl.doe.gov/smartgrid/.

[3] L. Xu, M. Y. Chow, J. Timmis and L. S. Taylor, "Power Distribution Outage Cause Identification With Imbalanced Data Using Artificial Immune Recognition System (AIRS) Algorithm," IEEE Transactions on Power Systems, vol. 22, no. 1, pp. 198-204, February 2007.

[4] D. M. Staszesky, D. Craig and C. Befus, "Advanced feeder automation is here," IEEE Power and Energy Magazine, pp. 56-63, Sept.-Oct. 2005.

[5] [Online]. Available: http://smartgrid.ieee.org/newsletters/april-2014/distributionautomation-and-the-self-healing-network.

[6] S. Chouhan, H. Wan, H. J. Lai, A. Feliachi and M. A. Choudhry, "Intelligent reconfiguration of smart distribution network using multi-agent technology," in 2009 IEEE Power \& Energy Society General Meeting, Calgary, AB, 2009.

[7] S. Chouhan, J. Ghorbani, H. Inan, A. Feliachi and M. A. Choudhry, "Smart MAS restoration for distribution system with Microgrids," in 2013 IEEE Power \& Energy Society General Meeting, Vancouver, BC, 2013.

[8] S. Chouhan, F. D. Mohammadi, A. Feliachi, J. M. Solanki and M. A. Choudhry, "Hybrid MAS Fault Location, Isolation, and Restoration for smart distribution system with Microgrids," in 2016 IEEE Power and Energy Society General Meeting (PESGM), Boston, MA, 2016. 
[9] S. Chouhan, H. Inan and A. Feliachi, "Optimal number and placement of automated sectionalizing switches for smart grid Distribution Automation," in 2016 IEEE Power and Energy Society General Meeting (PESGM), Boston, MA, 2016.

[10] S. Chouhan, H. Inan, "Automated Sectionalizing Switch Optimization for Smart Grid Distribution Automation," in CIGRE US National Committee Grid of the Future Symposium, Philadelphia, PA, 2016.

[11] J. Ghorbani, S. Chouhan, M. A. Choudhry, and A. Feliachi, "Hybrid multi agent approach for power distribution system restoration," in 2013 IEEE Energytech, Cleveland, OH, 2013.

[12] M. Baran, A. Padmanabhan, S. Chouhan, X. Yuan, J. Smith and H. Mayfield, "A model based fault locating method for distribution systems," in 2014 IEEE PES General Meeting | Conference \& Exposition, National Harbor, MD, 2014.

[13] G. Weiss, Multi-agent Systems: A Modern Approach to Distributed Artificial Intelligence, The MIT Press, 2000.

[14] D. A. Cartes and S. K. Srivastava, "Agent Applications and their Future in the Power Industry," in 2007 IEEE Power Engineering Society General Meeting, Tampa, FL, 2007.

[15] Zhong Zhang, J. D. McCalley, V. Vishwanathan and V. Honavar, "Multiagent system solutions for distributed computing, communications, and data integration needs in the power industry," in IEEE Power Engineering Society General Meeting, 2004.

[16] G. Levitin, S. Mazal-Tov, and D. Elmakis, "Genetic algorithm for optimal sectionalizing in radial distribution systems with alternative supply," Electr. Power Syst. Res., vol. 35, no. 3, pp. 149-155, 1995.

[17] R. Billinton and S. Jonnavithula, "Optimal switching device placement in radial distribution systems," IEEE Transactions on Power Delivery, vol. 11, no. 3, pp. 16461651, July 1996.

[18] P. M. S. Carvalho, L. A. F. M. Ferreira and A. J. C. da Silva, "A decomposition approach to optimal remote controlled switch allocation in distribution systems," IEEE Transactions on Power Delivery, vol. 20, no. 2, pp. 1031-1036, April 2005.

[19] Chao-Shun Chen, Chia-Hung Lin, Hui-Jen Chuang, Chung-Sheng Li, Ming-Yang Huang and Chia-Wen Huang, "Optimal placement of line switches for distribution 
automation systems using immune algorithm," IEEE Transactions on Power Systems, vol. 21, no. 3, pp. 1209-1217, August 2006.

[20] L. G. W. da Silva, R.A.F. Pereira, J.R. Abbad, and J.R.S. Mantovani, "Optimised placement of control and protective devices in electric distribution systems through reactive tabu search algorithm," Electr. Power Syst. Res., vol. 78, no. 3, p. 372-381, 2008.

[21] A Moradi, M. Fotuhi-Firuzabad, and M. Rashidi-Nejad, "A reliability cost/worth approach to determine optimum switching placement in distribution systems," in Proc. IEEE Power Eng. Soc. Asia Pacific Transmission Distribution Conf. Exhib, 2005.

[22] A. Moradi and M. F. Firuzabad, "Optimal switch placement in distribution systems using trinary particle swarm optimization algorithm," IEEE Trans. Power Del., vol. 23, no. 1, p. 271-279, Jan. 2008.

[23] H. Falaghi, M. R. Haghifam and C. Singh, "Ant Colony Optimization-Based Method for Placement of Sectionalizing Switches in Distribution Networks Using a Fuzzy Multiobjective Approach," IEEE Transactions on Power Delivery, vol. 24, no. 1, pp. 268-276, Jan. 2009.

[24] M. Kezunovic, "Smart Fault Location for Smart Grids," IEEE Transactions on Smart Grid, vol. 2, no. 1, pp. 11-22, March 2011.

[25] J. Mora-Florez, J. Melendez, and G. Carrillo-Caicedo, "Comparison of impedance based fault location methods for power distribution systems," Elect. Power Syst. Res., vol. 78, p. 657-666, 2008.

[26] J. Izykowski M. M. Saha and E. Rosolowski, Fault Location on Power Networks, 2010, Springer-Verlag, 2010.

[27] N. Karnik, S. Das, S. Kulkarni and S. Santoso, "Effect of load current on fault location estimates of impedance-based methods," in 2011 IEEE Power and Energy Society General Meeting, San Diego, CA, 2011.

[28] J. Ghorbani, M. Choudhry, and A. Feliachi, "Fault location and isolation using multi agent systems in power distribution systems with distributed generation sources," in Innovative Smart Grid Technologies (ISGT), 2013 IEEE PES, 2013. 
[29] G. C. Lampley, "Fault detection and location on electrical distribution system," in proc. of IEEE Rural Electric Power Conference, 2002.

[30] P. Verho M. M. Saha, R. Das and D. Novosel, "Review of Fault Location Techniques for Distribution Systems," proc. of Int. Conf on Power Systems and Communications Infrastructures," in proc. of Int. Conf on Power Systems and Communications Infrastructures, Beijing, 2002.

[31] D. Thukaram, H. P. Khincha and H. P. Vijaynarasimha, "Artificial neural network and support vector Machine approach for locating faults in radial distribution systems," IEEE Transactions on Power Delivery, vol. 20, no. 2, pp. 710-721, April 2005.

[32] N. Karnik S. Das, S. Kulkarni and S. Santoso, "Distribution fault location using shortcircuit fault current profile approach," in proc. of IEE PES GM, 2011.

[33] M. Michalik, W. Rebizant, M. Lukowicz, Seung-Jae Lee and Sang-Hee Kang, "Highimpedance fault detection in distribution networks with use of wavelet-based algorithm," IEEE Transactions on Power Delivery, vol. 21, no. 4, pp. 1793-1802, Oct. 2006.

[34] J. J. Mora, G. Carrillo and L. Perez, "Fault Location in Power Distribution Systems using ANFIS Nets and Current Patterns," in 2006 IEEE/PES Transmission \& Distribution Conference and Exposition: Latin America, Caracas, 2006.

[35] J. Mora-Florez, V. Barrera-Nunez and G. Carrillo-Caicedo, "Fault Location in Power Distribution Systems Using a Learning Algorithm for Multivariable Data Analysis," IEEE Transactions on Power Delivery, vol. 22, no. 3, pp. 1715-1721, July 2007.

[36] A.L. Morelato and A. Monticelli, "Heuristic search approach to distribution system restoration," IEEE Transactions on Power Delivery, vol. 4, no. 4, pp. 2235-2241, Oct. 1989.

[37] C. C. Liu, S. J. Lee and S. S. Venkata, "An expert system operational aid for restoration and loss reduction of distribution systems," IEEE Transactions on Power Systems, vol. 3, no. 2, pp. 619-626, 1988.

[38] W. Isamu and N. Makoto, "A genetic algorithm for optimizing switching sequence of service restoration in distribution systems," in Proc. Congr. Evolutionary Computation, 2004. 
[39] Y. Fukuyama, "Reactive Tabu Search for distribution load transfer operation," in Proc. IEEE Power Eng. Soc. Winter Meeting, 2000.

[40] K. H. Jung, H. Kim, and Y.Ko, "Network reconfiguration algorithm for automated distribution systems based on artificial intelligence," IEEE Trans. Power Del., vol. 8, no. 4, p. 1933-1941, Oct. 1993.

[41] S. Das, S. Bose, S. Pal, N. N. Schulz, C. M. Scoglio and B. Natarajan, "Dynamic reconfiguration of shipboard power systems using reinforcement learning," IEEE Transactions on Power Systems, vol. 28, no. 2, pp. 669-676, May 2013.

[42] R. Perez-Guerrero, G. T. Heydt, N. J. Jack, B. K. Keel and A. R. Castelhano, "Optimal Restoration of Distribution Systems Using Dynamic Programming," IEEE Transactions on Power Delivery, vol. 23, no. 3, pp. 1589-1596, July 2008.

[43] T. Nagata, Y. Tao, K. Kimura, H. Sasaki, and H. Fujita, "A multi-agent approach to distribution system restoration," in Circuits and Systems, 2004. MWSCAS '04. The 2004 47th Midwest Symposium, 2004.

[44] Fabio Luigi Bellifemine, Giovanni Caire, Dominic Greenwood, Developing MultiAgent Systems with JADE, Wiley, 2007.

[45] J. M. Solanki, S. Khushalani and N. N. Schulz, "A Multi-Agent Solution to Distribution Systems Restoration," IEEE Transactions on Power Systems, vol. 22, no. 3, pp. 10261034, 2007.

[46] T. Nagata and H. Sasaki, "A multi-agent approach to power system restoration," IEEE Transactions on Power Systems, vol. 17, no. 2, pp. 457-462, May 2002.

[47] T. Nagata, N. Iba, Y. Tao and H. Sasaki, "A new power system restoration framework by means of multi-agent approach," in IEEE/PES Transmission and Distribution Conference and Exhibition, 2002.

[48] T. Nagata, Y. Tao, Y. Tahara, T. Aoyama, H. Fujita and M. Koaizawa, "Development of bulk power system restoration simulator by means of multi-agent approach," in Circuits and Systems, 2004. MWSCAS '04. The 2004 47th Midwest Symposium on, 2004.

[49] Dong Liu, Yunping Chen, Guang Shen and Youping Fan, "A Multi-Agent Based Approach for Modeling and Simulation of Bulk Power System Restoration," in 2005 
IEEE/PES Transmission \& Distribution Conference \& Exposition: Asia and Pacific, Dalian, 2005.

[50] Belkacemi, Rabie, and Ali Feliachi, "Multi-agent design for power distribution system reconfiguration based on the artificial immune system algorithm," in Circuits and Systems (ISCAS), Proceedings of 2010 IEEE International Symposium on, 2010.

[51] Belkacemi, Rabie, Ali Feliachi, M. A. Choudhry, and J. E. Saymansky, "Multi-Agent systems hardware development and deployment for smart grid control applications," in Power and Energy Society General Meeting, 2011.

[52] M. J. Ghorbani, M. A. Choudhry and A. Feliachi, "A Multiagent Design for Power Distribution Systems Automation," IEEE Transactions on Smart Grid, vol. 7, no. 1, pp. 329-339, Jan 2016.

[53] P. Stone and M. Velos, "Multiagent systems: a survey from the machine learning perspective," Autonomous Robots, vol. 8, no. 3, pp. 345-383, 2000.

[54] R. Sutton and A. Barto, Reinforcement Learning: An Introduction, The MIT Press, 1998.

[55] K. S. Narendra and M. A. L. Thathachar, Learnng Automata: An Introduction, Prentice Hall, 1989.

[56] C. J. C. H. Watkins, and P. Dayan, "Q-learning," Machine Learning, vol. 8, no. 3, pp. 279-292, May 1992.

[57] D. Ye, M. Zhang and D. Sutanto, "A Hybrid Multiagent Framework With Q-Learning for Power Grid Systems Restoration," IEEE Transactions on Power Systems, vol. 26, no. 4, pp. 2434-2441, Nov. 2011.

[58] J. Jung, Chen-Ching Liu, S. L. Tanimoto and V. Vittal, "Adaptation in load shedding under vulnerable operating conditions," IEEE Transactions on Power Systems, vol. 17, no. 4, pp. 1199-1205, Nov. 2002.

[59] "IntelliTeam SG Automatic Restoration System," S\&C Electric Company, [Online]. Available: http://www.sandc.com/products/automation-control/.

[60] M. Wooldridge, N. R. Jennings, "Intelligent agents: Theory and Practice," The Knowledge Engineering Review, vol. 10, no. 2, p. 115 - 152, 1995. 
[61] S. Russell and P.Norvig, Artificial Intelligence: A modern approach, Printice-Hall, 1995.

[62] Donald A. Waterman; Frederick Hayes-Roth; Douglas B. Lentat, Building Expert Systems, Reading, MA : Addison-Wesley Publishing Co, 1983.

[63] P.Jackson, Introduction to Expert Systems, Reading, M.A: Addison-Wesley, 1986.

[64] John R Searle, Speech Acts: An essay in the philosophy of Language, Cambridge U. Press, 1970.

[65] "The Foundation for Physical Intelligent Agents," [Online]. Available: http://www.fipa.org.

[66] "FIPA 97 specifications, version 2.0, ACL," [Online]. Available: http://www.fipa.org.

[67] C. S. Chen, C. H. Lin, H. J. Chuang, C. S. Li, M. Y. Huang, and C. W. Huang, "Optimal placement of line switches for distribution automation systems using immune algorithm," IEEE Trans. Power Syst., vol. 21, no. 3, p. 1209-1217, 2006.

[68] W. H. Kersting, "Radial Distribution Test Feeders," IEEE Trans. On Power Systems, vol. 6, no. 3, Aug 1991.

[69] C.A. Plet, M. Graovac, R. Iravani, T.C. Green, "Fault response of grid-connected inverter dominated networks," in IEEE Power and Energy Society General Meeting, 2010 .

[70] J. Keller, B. Kroposki, "Understanding Fault Characteristics of Inverter-Based Distributed Energy Resources," Natl.Renew. Energy Lab, 2010.

[71] M. Baran, I. El-Markaby, "Fault analysis on distribution feeders with distributed generators," IEEE Trans. Power Syst., vol. 20, no. 4, p. 1757-1764, 2005.

[72] B. Kroposki, Optimization of Distributed and Renewable Energy Penetration in Electric Power Distribution Systems, Thesis Submitted to CSM, Golden, Colorado, 2008.

[73] IEEE Std 141-1993 Red Book - Electric Power Distribution for Industrial Plants, IEEE, 1993. 
[74] IEEE. IEEE 1547.2-2008. Standard for Interconnecting Distributed Resources with Electric Power Systems, IEEE, 2008.

[75] C. A. Plet, T. C. Green, "Fault response of inverter interfaced distributed generators in grid-connected applications," Elect. Power Syst. Res., vol. 106, pp. 21-28, Jan. 2014.

[76] R. Lasseter, "Dynamic models for micro-turbines \& fuel cells," in Proc. IEEE Power Eng. Soc. Summer Meeting, 2001.

[77] M. Baran and I. El-Markabi, "An EMTP based simulator for distribution systems with distributed generation," in Proc. Power System Conf. Impact Distributed Generation, Clemson, SC, 2002.

[78] IEEE Recommended Practice for Utility Interface of Photovoltaic (PV) Systems, IEEE std. 929-2000, IEEE, 2000.

[79] T. S. Basso, and R. DeBlasio, "IEEE 1547 series of standards: interconnection issues," IEEE Transactions on Power Electronics, vol. 19, no. 5, p. 1159 - 1162, 2004.

[80] IEEE Standard for Electrical Power System Device Function Numbers, Acronyms and Contact Designation, IEEE Std. C37.2, IEEE, 2008.

[81] K. N. Miu, Hsiao-Dong Chiang, Bentao Yuan and G. Darling, "Fast service restoration for large-scale distribution systems with priority customers and constraints," IEEE Transactions on Power Systems, vol. 13, no. 3, pp. 789-795, 1998.

[82] W. H . Kersting, Distribution system modeling and analysis, 2 ed., Boca Raton, Florida: Taylor \& Francis Group, 2006, p. 45.

[83] L. Busoniu, R. Babuska, and B. De Schutter, "A comprehensive survey of multiagent reinforcement learning," IEEE Trans. Systems, Man, and Cybernetics, Part C: Applications and Reviews, vol. 38, no. 2, pp. 156-172, 2008. 